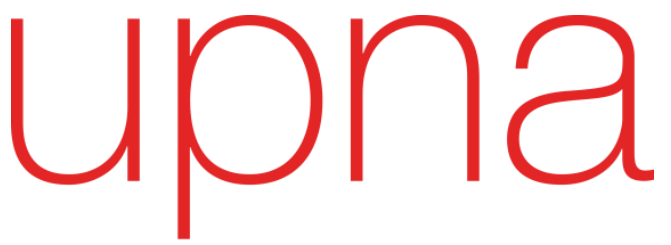

Universidad Pública de Navarra

Nafarroako Unibertsitate Publikoa

\title{
RIESGO DE HEMORRAGIA DIFERIDA TRAS RESECCIÓN ENDOSCÓPICA MUCOSA DE LESIONES COLORRECTALES DE GRAN TAMAÑO
}

EDUARDO ALBÉNIZ ARBIZU 
upna 


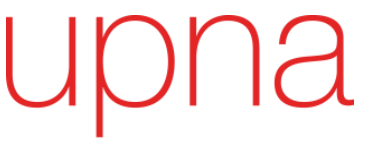

Universidad Pública de Navarra

Nafarroako Unibertsitate Publikoa

\section{RIESGO DE HEMORRAGIA DIFERIDA TRAS RESECCIÓN ENDOSCÓPICA MUCOSA DE LESIONES COLORRECTALES DE GRAN TAMAÑO}

Memoria presentada por: Eduardo Albéniz Arbizu para aspirar al grado de doctor por la Universidad Pública de Navarra.

El tutor de este trabajo ha sido:

Roberto Aguado Jiménez

El trabajo ha sido realizado en la modalidad de compendio de publicaciones en:

Complejo Hospitalario de Navarra. Servicio de Aparato Digestivo. Unidad de Endoscopia. Navarrabiomed-UPNA-IDISNA

Línea de Investigación:

Línea 2. Salud Pública, Enfermería y Medicina 
upna 
Este trabajo ha sido posible gracias a la beca de intensificación de "La Fundación la Caixa/Caja Navarra" (ID 100010434; Proyecto PR15/11100006), a una beca de la Fundación de la Sociedad Española de Endoscopia Digestiva (FSEED) y al soporte de Navarrabiomed y del Servicio de Digestivo y Unidad de Endoscopia del Complejo Hospitalario de Navarra. 
upna 


\section{AGRADECIMIENTOS}

"Me contaron una y otra vez como era y como debía ser la vida...incluso como era la muerte, pero no me contaron que cada uno debe vivir la suya, y que a veces no hay normas..."

Muchos acontecimientos ocurren de forma inesperada, pero en ocasiones, es uno mismo el que los busca con intensidad. Esta tesis, fruto del trabajo personal y colaborativo ha sido muy buscada, lo cual produce una gran satisfacción.

No hubiera sido posible sin el amor y estabilidad que me proporciona mi familia; mi mujer Natalia y mis hijos Marina y Javier; mi madre Consuelo y mis cuatro hermanos. Gracias, os quiero.

No hubiera sido posible sin la ayuda de mis compañeros del grupo de trabajo de resección endoscópica de la Sociedad Española de Endoscopia Digestiva (GSEEDRE). Gracias de verdad.

Y sobre todo no hubiera sido posible sin la ayuda de determinados compañeros y amigos, los cuales detallo a continuación: María Fraile, Charly Guarner, Zeben Gimeno, Marco Antonio Álvarez, Berta Ibáñez, Mónica Enguita y Roberto Aguado. Cada uno de ellos, hizo grandes aportaciones que han quedado plasmadas en las publicaciones de esta tesis. Gracias y mil gracias de nuevo.

¡Qué bien sienta terminar algo y sentirse agradecido!

Gracias 
upna 


\section{TABLA DE CONTENIDO}

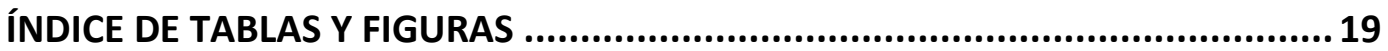

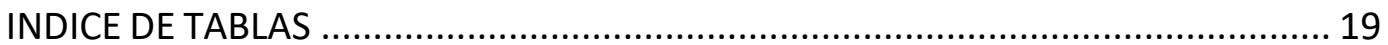

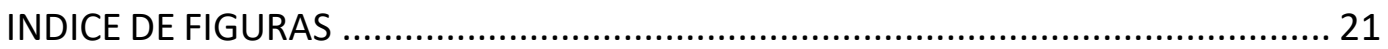

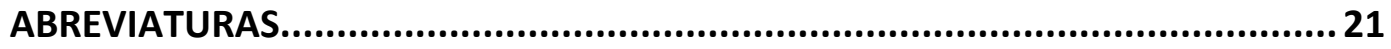

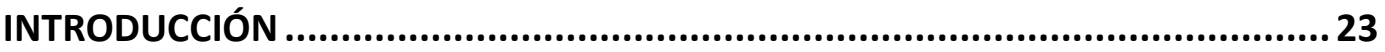

I.1 ANTECEDENTES: RESECCIÓN MUCOSA ENDOSCÓPICA COLORRECTAL............. 23

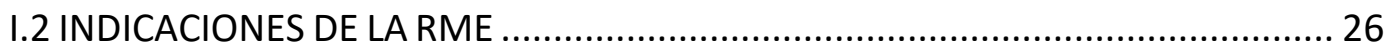

I.3 COMPLICACIONES DE LA RME: HEMORRAGIA DIFERIDA............................. 30

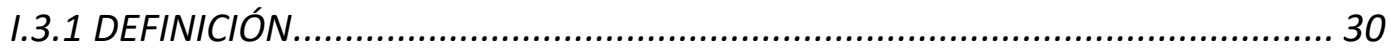

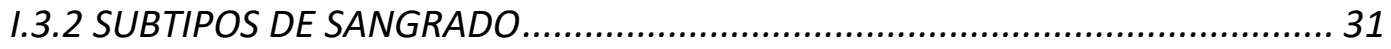

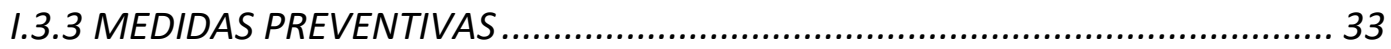

I.3.4 TRATAMIENTO DE LA HEMORRAGIA DIFERIDA ......................................... 36

I.4 JUSTIFICACIÓN DE LA INVESTIGACIÓN REALIZADA EN ESTA TESIS .................... 38

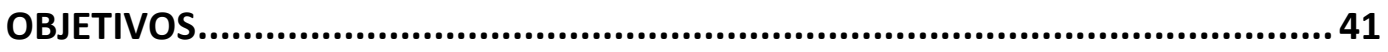

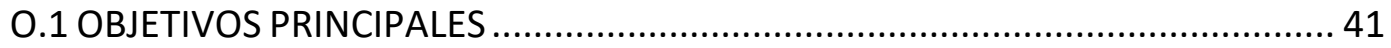

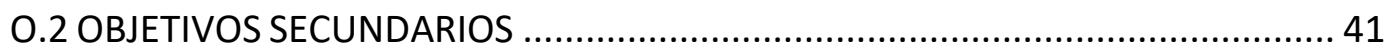

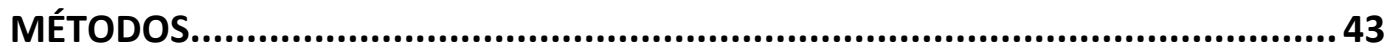

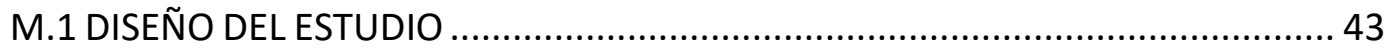

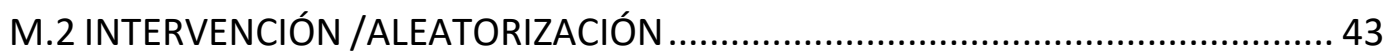

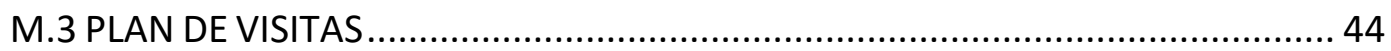

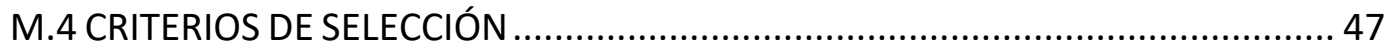

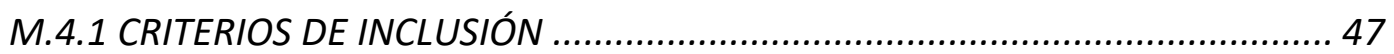

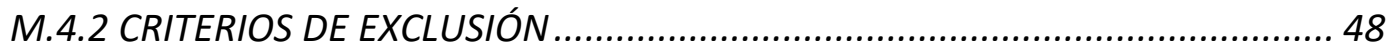

M.5 VARIABLES E INSTRUMENTOS DE MEDICIÓN ................................................. 50

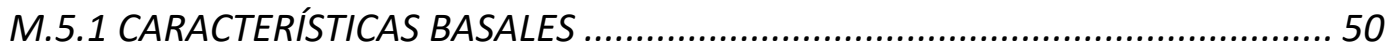

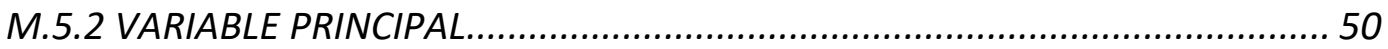

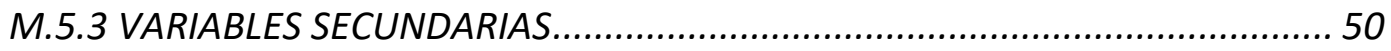


M.6 ANÁLISIS ESTADÍSTICO

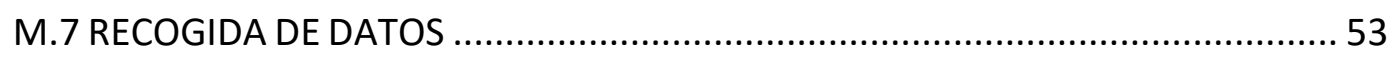

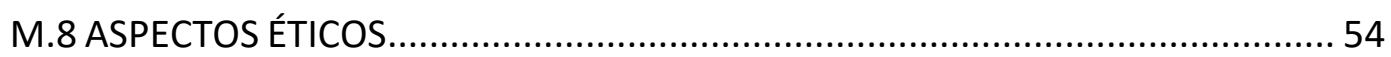

ARTÍCULO PRELIMINAR 1: Modelo predictivo del riesgo de hemorragia diferida tras la RME de lesiones colorrectales de gran tamaño. ..................................55

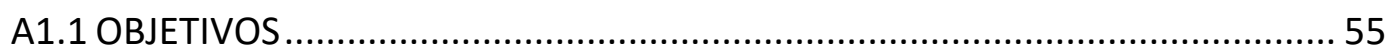

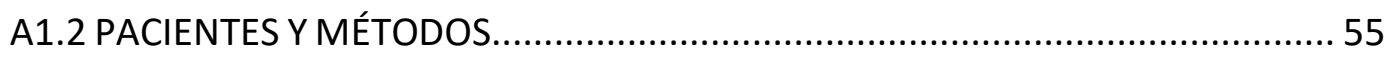

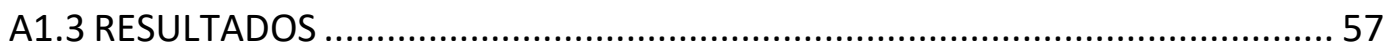

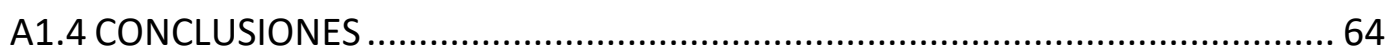

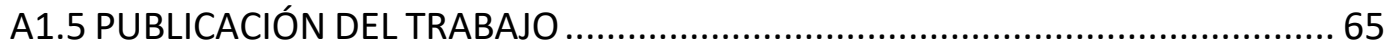

ARTÍCULO 2. Validación clínica de los modelos predictores del riesgo de hemorragia diferida tras RME de lesiones colorrectales de gran tamaño. .......67 67

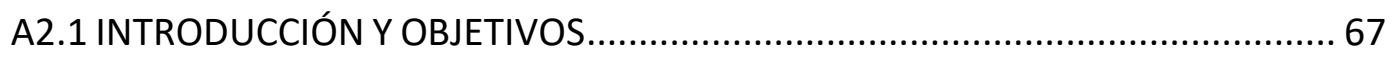

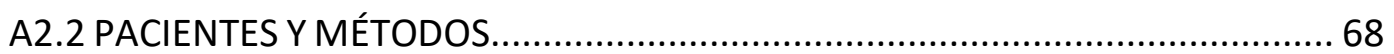

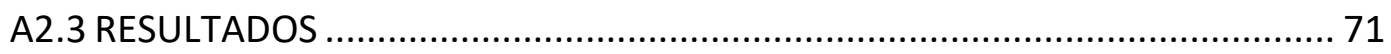

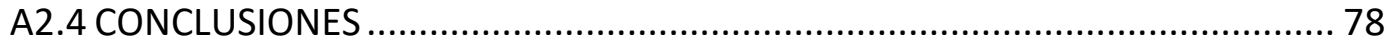

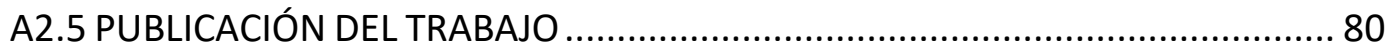

ARTíCULO 3. Cierre completo con hemoclips tras RME de lesiones colorrectales con riesgo sustancial de sangrado. Ensayo clínico aleatorizado......................8 81

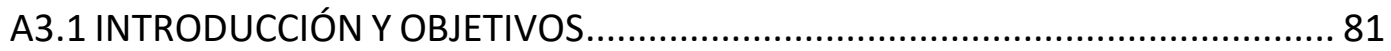

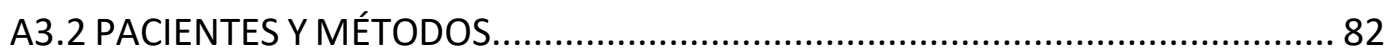

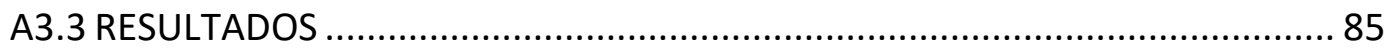

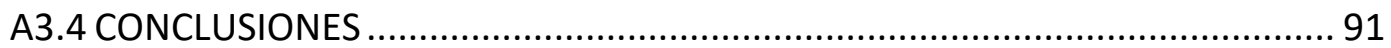

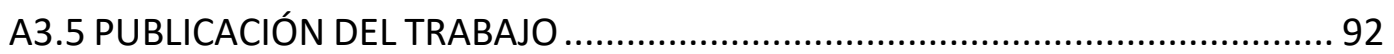

ARTíCULO 4. Cierre con clips para la prevención de hemorragia diferida tras

RME colorrectal. Metaanálisis de ensayos clínicos randomizados. ..................93 93

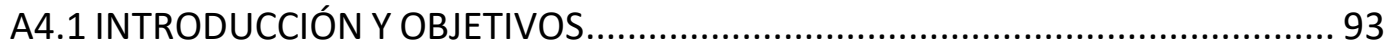

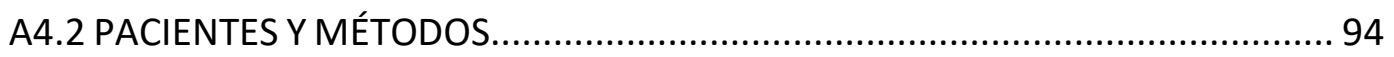

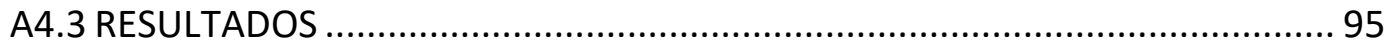

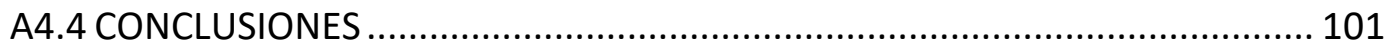


A4.5 PUBLICACIÓN DEL TRABAJO

ARTícULO 5. La respuesta a "cuándo cerrar con clips" después de la RME

colorrectal, basada en un análisis de coste-efectividad. 103

A5.1 INTRODUCCIÓN Y OBJETIVOS. 103

A5.2 PACIENTES Y MÉTODOS. 104

A5.3 RESULTADOS 107

A5.4 CONCLUSIONES 115

A5.5 PUBLICACIÓN DEL TRABAJO 116

9. DISCUSIÓN. 117

10. RESUMEN DE CONCLUSIONES GENERALES 135 CONCLUSIONES FINALES 137

11. REFERENCIAS 139

12. APÉNDICE 155

12.1 PUBLICACIONES 155 
upna 


\section{ÍNDICE DE TABLAS Y FIGURAS}

\section{INDICE DE TABLAS}

Tabla I.1. Algoritmo de tratamiento endoscópico para las lesiones colorrectales propuesto por el GSEED-RE (pg.30).

Tabla M.1: Score de riesgo de HD GSEED-RE (Albéniz et al. Clin Gastroenterol Hepatol 2016) (pg. 51).

Tabla M.2: Categorización de riesgo en pacientes según GSEED-RE (pg. 51).

Tabla A1.1: Características descriptivas de la muestra (1255 RME en 1214 pacientes) (pg. 60).

Tabla A1.2: Resultados de la regresión logística univariante para identificación de factores asociados a HD post-resección (pg. 62).

Tabla A1.3: Resultados de regresión logística multivariante incluyendo predictores independientes de HD post-resección (pg. 63).

Tabla A1.4: Probabilidad de HD en función del valor del score (pg. 64).

Tabla A1.5: Valores de puntuación de los factores de riesgo de HD (pg. 65).

Tabla A2.1: Características descriptivas de la cohorte post-2015 (pg. 74-75).

Tabla A2.2: Valores de puntuación utilizados en los scores M1 (GSEED-RE), M2 (ACER) y M3 (GSEEDE-RE2) (pg. 76).

Tabla A2.3: Resumen del rendimiento de los modelos para la cohorte post-2015, cohorte pre-2015 y la cohorte total (pg. 77).

Tabla A2.4: Probabilidad de HD en las categorías de riesgo bajo, medio y alto para las diferentes puntuaciones en la cohorte post-2015, pre-2015 y total (pg. 78).

Tabla A3.1. Características basales de los pacientes asignados a cada grupo (pg. 88). 
Índice de tablas y figuras

Tabla A3.2. Resultados primarios y secundarios del análisis ITT y PP (pg. 90).

Tabla A3.3. Análisis de HD por subgrupos de factores de riesgo (pg. 92).

Tabla A4.1 - Características de los estudios (pg. 98).

Tabla A4.2 Metaanálisis del riesgo general de HD - Resultados de la metaregresión (pg. 100).

Tabla A5.1. Parámetros asumidos para el estudio CE (pg. 110).

Tabla A5.2. Análisis de costes y coste-efectividad del cierre profiláctico con clips en una estrategia universal y selectiva (pg. 111).

Tabla A5.3. Análisis del umbral de coste-efectividad (pg. 116). 


\section{INDICE DE FIGURAS}

Figura I.1. Subtipos de RME (pg. 25).

Figura I.2. Imágenes de RME en fragmentos de gran adenoma velloso en sigma (pg. 25).

Figura I.3. Hemorragia diferida y tratamiento con hemoclips (pg. 33).

Figura I.4. Cierre completo del defecto mucoso tras RME de LST-G (pg. 35).

Figura A1.1: Curva de calibración para el modelo de regresión multivariante (pg. 64).

Figura A2.1. Nuevo Score de riesgo de hemorragia diferida para la RME de LCRNP de gran tamaño: GSEED-RE2 (pg. 78).

Figura A3. 1. Diagrama de flujo (pg. 85).

Fig. A3.2. Riesgo de HD en el análisis por intención de tratar (ITT) (control vs. tratados) y en el análisis por protocolo (PP) (control vs. cierre completo) (pg. 89).

Figura A4.1: Diagrama de bosque que compara el brazo de cierre con clips vs grupo control (pg. 97).

Figura A4.2. Análisis de subgrupos del riesgo relativo de desarrollar $\mathrm{HD}$ en el grupo de tratamiento y control para pólipos grandes versus pequeños (pg. 99).

Figura A4.3. Análisis de subgrupos que compara el riesgo de HD del grupo control y el de tratamiento para lesiones ubicadas distal y proximalmente (pg. 100).

Figura A5.1. Árbol de decisiones (pg. 105).

Figura A5.2. Riesgo de HD vs ICER para las estrategias de cierre universal y selectivo en los contextos económicos de Europa (A) y Norteamérica (B) (pg. 110).

Figura A5.3. Riesgo de HD vs coste de un episodio de HD para las estrategias de cierre universal y selectivo en los contextos económicos de Europa (A) y Norteamérica (B) (pg. 111). 
Figura A5.4. Riesgo de HD vs coste de un clip para las estrategias de cierre universal y selectivo en los contextos económicos de Europa (A) y Norteamérica (B) (pg. 111).

Figura A5.5. Riesgo de HD vs intervalo entre clips para las estrategias de cierre universal y selectivo en los contextos económicos de Europa $(A)$ y Norteamérica (B) (pg. 112).

Figura A5.6. Riesgo de HD vs coste del cierre completo con clips para las estrategias de cierre universal y selectivo en los contextos económicos de Europa (A) y Norteamérica (B) (pg. 112).

Figura A5.7. Riesgo de HD vs RRR de HD tras aplicar clips para las estrategias de cierre universal y selectivo en los contextos económicos de Europa (A) y Norteamérica (B) (pg. 113).

Figura A5.8. Fórmula matemática que relaciona todos los parámetros (pg. 113). 


\section{ABREVIATURAS}

ACER (del inglés, "Australian Colonic Endoscopic Resection Study-Group"): Grupo Australiano de resección mucosa endoscópica colónica.

AINES: Antinflamatorios no esteroideos.

ANOVA: Análisis de la varianza.

ARD (del inglés "Absolute risk difference"): Diferencia del riesgo absoluta.

ASA (del inglés, "American Society of Anesthesiologists"): Clasificación de la Sociedad Americana de Anestesiología del riesgo anestésico.

APC (del inglés “Argon Plasma Coagulation”): Coagulación con plasma de argón.

AUC (del inglés "Area under the curve"): Área bajo la curva.

CE: Coste-efectividad/coste-efectivo.

CEIC: Comité de ética de la Investigación Clínica.

CRDe: Cuaderno de recogida de datos electrónico.

CS (del inglés "cost-saving"): Ahorro de costes.

DSE: Disección Submucosa Endoscópica.

FNS (del inglés "Funds nedeed to spend"): Fondos necesarios para gastar.

GSEED-RE: Grupo de Resección Endoscópica de la Sociedad Española de Endoscopia Digestiva.

HD (DB, delayed bleeding en inglés): Hemorragia diferida.

HR (del inglés "High risk"): Riesgo alto.

IC: Intervalo de confianza.

ICER: Incremento de la ratio coste-efectividad.

ITT: Intención de tratar.

INR: Índice internacional normalizado. 


\section{Abreviaturas}

LCRNP: Lesiones colorrectales no pediculadas.

LST (del inglés "Laterally Spreading Tumor"): Lesiones superficiales de extensión lateral.

LST-G: Lesiones superficiales de extensión lateral granulares.

LST-GH: Lesiones superficiales de extensión lateral granulares homogéneas.

LST-GM: Lesiones superficiales de extensión lateral granulares mixtas.

LST-NG: Lesiones superficiales de extensión lateral no granulares.

LST-NG PD: Lesiones superficiales de extensión lateral no granulares planas pseudodeprimidas (lesiones pseudodeprimidas).

LST-NG PE: Lesiones superficiales de extensión lateral no granulares planas elevadas (lesiones elevadas).

NBI (del inglés "Narrow Band Imaging").

NOAC: Nuevos anticoagulantes orales (Ej. Dabigatrán).

OR (del inglés, “Odds ratio”): Razón de odds.

OTSC (del inglés "Over The Scope Clip"): Clips de gran tamaño premontados sobre capuchones.

PP: Por protocolo.

QALYs (o AVACs): Años de vida ajustados por calidad.

RME (EMR en inglés): Resección endoscópica mucosa.

ROC: Curva de característica operativa del receptor.

RRR (del inglés “Relative risk reduction”): Reducción relativa del riego.

SPP: Síndrome postpolipectomía.

SPSS: "Statistical Package for the Social Sciences".

TC (del inglés "Total cohort"): Cohorte total.

TTSC (del inglés "Through The Scope Clip"): Clip aplicados a través del endoscopio. 


\section{INTRODUCCIÓN}

\section{I.1 ANTECEDENTES: RESECCIÓN MUCOSA ENDOSCÓPICA}

\section{COLORRECTAL}

La resección mucosa endoscópica (RME) o mucosectomía es una de las técnicas endoscópicas que han modificado el panorama terapéutico de las neoplasias superficiales del tracto gastrointestinal, constituyendo una alternativa eficaz y segura al tratamiento quirúrgico convencional, con la consiguiente reducción en morbilidad y $\operatorname{costes}^{1-3}$.

En 1955, Rosemberg utilizó la inyección de suero salino submucoso para asistir polipectomías con un sigmoidoscopio rígido y posteriormente, en 1973, fue Dehyle quien la desarrolló con endoscopios flexibles ${ }^{4,5}$.

En el año 1984 autores japoneses describieron un nuevo método de resección endoscópica de lesiones neoplásicas superficiales o no avanzadas del tubo digestivo que denominaron "stripbiopsy". Esta técnica aprovechaba el origen embriológico diferente de la mucosa y submucosa, provenientes del endodermo, del de la muscular y la serosa, cuyo origen es el mesodermo. Al estar la submucosa compuesta por un tejido conectivo laxo, la inyección de suero salino separaba con facilidad las capas superficiales de las profundas, formando un colchón líquido entre la mucosa y la muscular propia que permitía la extirpación endoscópica más segura de las lesiones con un asa de polipectomía ${ }^{6}$. Esta técnica es conocida hoy en día como RME o mucosectomía ${ }^{1-3}$. 


\section{Introducción}

La RME constituye en la actualidad el método de elección para la extirpación de lesiones neoplásicas benignas o malignas que afectan exclusivamente a la mucosa o a la submucosa superficial. El desarrollo de las técnicas de imagen endoscópica como la alta definición, la magnificación y la cromoendoscopia virtual, han permitido una caracterización precisa de la naturaleza de las lesiones, un diagnóstico in vivo benigno o maligno y la evaluación del grado de infiltración en profundidad, aspectos imprescindibles antes de intentar la $\mathrm{RME}^{7-15}$.

En la actualidad, el cáncer de colon y recto constituye el tercer tumor maligno por incidencia y la segunda causa de muerte por cáncer ${ }^{16}$. La reducción de la incidencia y la mortalidad del cáncer colorrectal que permite la extirpación endoscópica de las lesiones precursoras de esta neoplasia ha propiciado un mayor desarrollo e interés de la RME para las lesiones superficiales de colon y recto $^{17,18}$.

Existen varias formas de hacer la RME, la RME simple asistida por inyección, la que utiliza pinzas de tracción, la asistida por capuchones y la que se realiza con capuchón más bandas elásticas (Figura I.1). La variante más utilizada es la RME asistida por inyección (Figura I.2) en la que ayudados por una aguja, situada en el extremo de una vaina flexible que se introduce por el endoscopio, se inyectan sustancias de diferentes densidades en la capa submucosa, resecándose posteriormente la lesión neoplásica con un asa de polipectomía. En pólipos menores de dos centímetros se suelen conseguir resecciones en un solo fragmento; es lo que se denomina resección en bloque ${ }^{19-25}$. 
Figura I.1. Subtipos de RME.

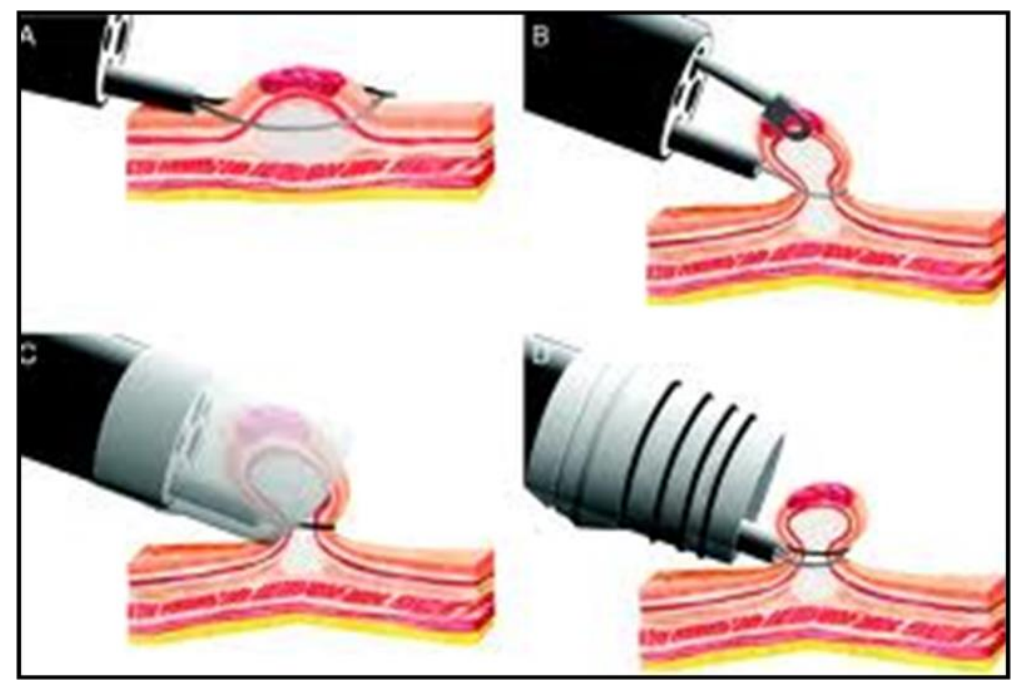

A) RME asistida por inyección. B) "Strip-biopsy" o RME asistida con pinzas de tracción. C) RME con capuchón. D) RME con capuchón y bandas elásticas. Imagen adaptada de Thoracickey.com.

Figura I.2. Imágenes de RME en fragmentos de gran adenoma velloso en sigma.
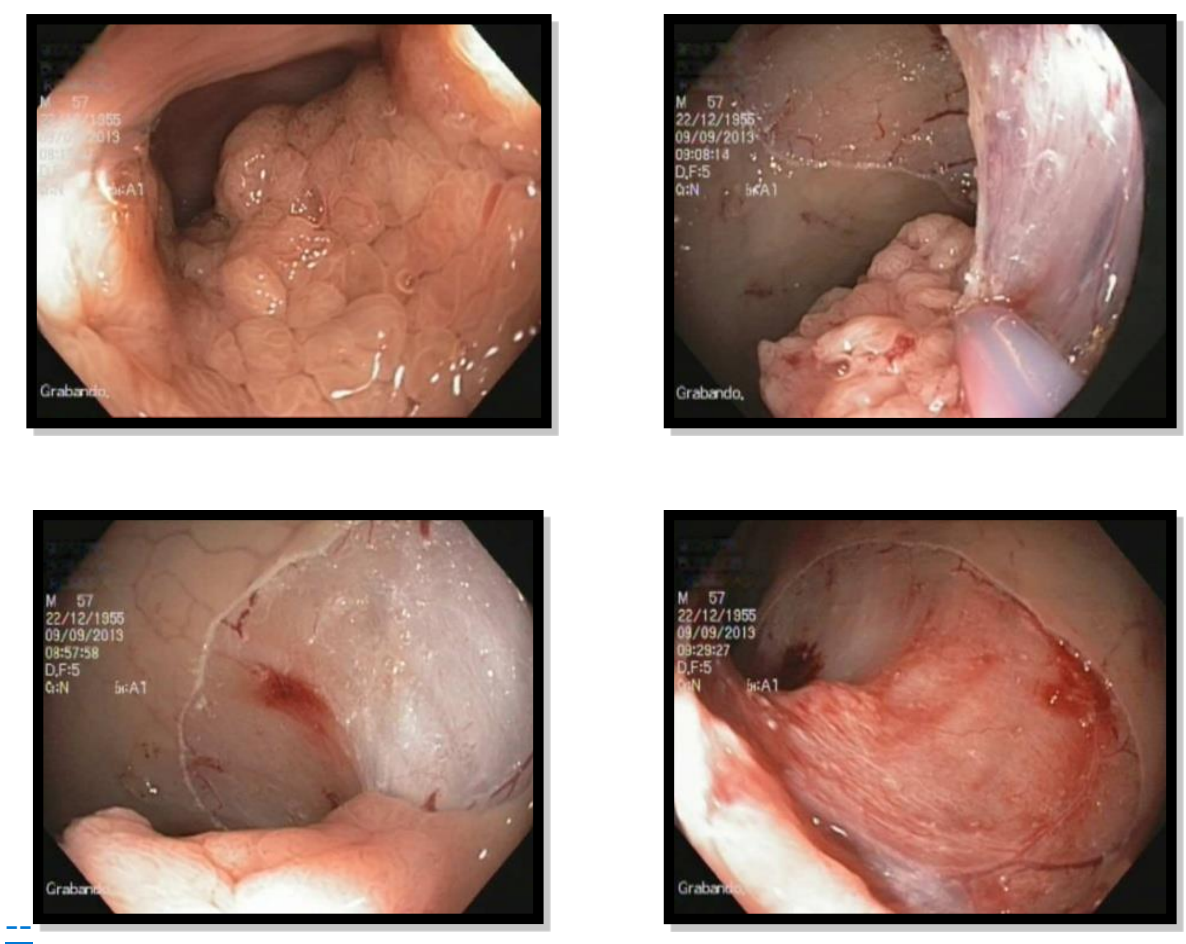
Introducción

Las lesiones mayores de 20 o $30 \mathrm{~mm}$ raramente se pueden resecar en una sola pieza y deben por tanto resecarse en fragmentos; este tipo de resección se llama RME en fragmentos o piecemeal ${ }^{25}$.

Existe además otra nueva variante llamada RME "underwater" o mucosectomía en inmersión. Esta técnica permite realizar la resección sin inyección submucosa. Una vez identificada la lesión, se aspira el aire y se inyecta agua hasta que la luz colónica está completamente replecionada. Se marcan los márgenes de la lesión debajo del agua con argón plasma y posteriormente se realiza la resección mucosa. Algunos estudios han mostrado que es una técnica efectiva y segura con un bajo índice de sangrado. La tasa de resección en bloque podría ser mayor que con la mucosectomía tradicional, aunque el tamaño grande de las lesiones constituye una limitación de esta variante técnica ${ }^{26-29}$.

\section{I.2 INDICACIONES DE LA RME}

La RME es una técnica establecida para abordar lesiones superficiales colorrectales generalmente no pediculadas (LCRNP), y con baja sospecha de invasión submucosa profunda o de invasión linfática ${ }^{8-12,30-34}$. En estos casos, la RME se considera de elección frente a las técnicas quirúrgicas, tanto desde un punto de vista clínico como económico ${ }^{32,33}$.

Un gran consenso internacional realizado en el año 2002 en París clasifica las neoplasias superficiales en pediculadas (0-Ip), protuídas o sésiles (0-Is), planas (planas superficialmente elevadas (0-Ila), completamente planas (0-IIb), planas con discreta depresión (0-IIc)) y excavadas (0-III). ${ }^{9}$ 
La RME ha demostrado ampliamente ser una técnica efectiva de resección de lesiones sésiles (0-Is) y planas (0-Ila/Ilb/Ilc) mayores de $5-10 \mathrm{~mm}$ y hasta un tamaño de 130 mm en algunos trabajos o sin limitación de tamaño en otros ${ }^{35,36}$

Las lesiones planas mayores de $10 \mathrm{~mm}$ se denominan lesiones superficiales de extensión lateral, conocidas por su término en inglés "Laterally Spreading Tumor" (LST). Se distinguen 2 subtipos: LST-G (granulares) y LST-NG (no granulares) $^{36-42}$.

Existen dos tipos de lesiones granulares, aquellas llamadas granulares homogéneas (LST-GH), compuestas por pequeños nódulos de tamaño bastante similar y que presentan un bajo riesgo de adenocarcinoma invasor $(0.5 \%)$ y las llamadas granulares mixtas (LST-GM), que albergan nódulos heterogéneos en tamaño. Los LST-GM con nódulos grandes mayores de $1 \mathrm{~cm}$ pueden tener una incidencia de cáncer al menos submucoso, del 10.5\%. La mayoría de las lesiones granulares pueden resecarse mediante RME, aunque cuando existen nódulos de gran tamaño es necesario resecar y analizar histológicamente el nódulo de mayor tamaño por separado ${ }^{39-41}$. Desde hace unos años, se está implementando en occidente otra técnica importada de Japón, llamada Disección Submucosa Endoscópica (DSE), que permite resecar la mayoría de las lesiones en bloque. Sin embargo, la diseminación de esta técnica es compleja por la complicada curva de aprendizaje que presenta ${ }^{40,41}$.

Los LST-NG se subdividen morfológicamente en lesiones planas elevadas (LST-NG PE) y pseudodeprimidas (LST-NG PD); las primeras presentan invasión submucosa en un $4.9 \%$ y las segundas hasta en un $31.6 \%$, aunque todos estos 


\section{Introducción}

porcentajes varían según las series, las cuales proceden en su mayoría de países orientales. Autores japoneses han propuesto algoritmos de tratamiento que limitan prácticamente la RME a las lesiones no granulares menores de $20 \mathrm{~mm}^{40,41}$.

Sin embargo, los trabajos de varios grupos occidentales proponen como más apropiado considerar separadamente las lesiones planas elevadas y las pseudodeprimidas, debido a que la mayoría de las lesiones planas elevadas podrían extirparse mediante RME en fragmentos y las segundas sí que precisan resección en bloque preferiblemente mediante DSE siempre que no exista sospecha de invasión submucosa profunda. Las series orientales más tradicionales no citan a las lesiones serradas, las cuales adoptan una morfología plana elevada en gran parte de las ocasiones ${ }^{14,15,40,41}$.

Como un buen resumen acerca de las indicaciones de la RME y sus variantes y de la DSE, se podría considerar el algoritmo de tratamiento propuesto por el Grupo de Resección Endoscópica de la Sociedad Española de Endoscopia Digestiva (GSEED-RE) que se plasmó en la guía clínica para la resección endoscópica de los pólipos del colon y del recto ${ }^{14,15}$ (Tabla I.1). Cabe destacar que el autor de esta tesis es el coordinador del GSEED-RE y junto con la Dra. Pelliséideó, coordinó y colaboró en la redacción de todos los capítulos que aparecen en la citada guía clínica y de la que se extrae gran parte de esta introducción. 
Tabla I.1. Algoritmo de tratamiento endoscópico para las lesiones colorrectales propuesto por el GSEED-RE.

\begin{tabular}{|l|l|l|}
\hline & \multicolumn{2}{|c|}{ ESTRATEGIA } \\
\hline SUBTIPO & $\begin{array}{l}\text { Tamaño } \\
\text { MORFOLÓGICO }\end{array}$ & $\begin{array}{l}\text { Tamaño } \\
>\mathbf{2 0} \mathbf{2 0} \mathbf{3 0 m m}\end{array}$ \\
\hline LST-GH & RME & $\begin{array}{l}\text { RME (DSE en los pocos casos } \\
\text { con sospecha de invasión } \\
\text { submucosa profunda y centros } \\
\text { con experiencia) }\end{array}$ \\
\hline LST-GM & RME & $\begin{array}{l}\text { Individualizar casos (RME /DSE: } \\
\text { si }\end{array}$ \\
\hline LST-NG PE * & RME, resecar/analizar \\
& nódulos grandes por separado) \\
\hline LST-NG PD* & RME & $\begin{array}{l}\text { Individualizar casos } \\
\text { (RME /DSE) }\end{array}$ \\
\hline Ols & Polipectomía/RME & Individualizar casos (RME /DSE) \\
\hline Non-lifting sign & Individualizar casos (RME, RME híbrida, RME Underwater, \\
& RME +avulsión o APC, DSE, cirugía) \\
\hline & Polipectomía & $\begin{array}{l}\text { Polipectomía/ RME (pedículos } \\
\text { grandes } \\
\text { semipediculadas) }\end{array}$ \\
\hline
\end{tabular}

RME: resección endoscópica mucosa; DSE: disección submucosa endoscópica; LST: lesión de extensión lateral; LST-GH: granular homogéneo; LST-GM: granular mixto; LST-NG PE: no granular plano elevado (elevadas); LST-NG PD: no granular pseudodeprimido (pseudodeprimidas). APC: Coagulación con plasma de argón. *A tener en cuenta que los pólipos serrados pueden suelen ser planos. En este caso, la RME en fragmentos es en general la técnica de elección. 
Introducción

\section{I.3 COMPLICACIONES DE LA RME: HEMORRAGIA DIFERIDA}

Pese a su gran eficacia y aceptable perfil de seguridad, la RME presenta complicaciones nada desdeñablesentre las que destacan la hemorragia, la perforación, el dolor, el síndrome post-polipectomía (SPP), la fiebre postresección, las derivadas de la sedación o de la anestesia general, aquellas secundarias a la preparación de la colonoscopia y las que surgen como consecuencia de la retirada o sustitución del tratamiento antiagregante o anticoagulante. Existen otras complicaciones infrecuentes que incluyen eventos cardiovasculares (angina, infarto, trastornos del ritmo cardiaco), bacteriemia estenosis cicatricial, rotura del bazo o alteración de la funcionalidad anorrectal $^{43,44,53-62,45,63-69,46-52}$.

Las complicaciones más importantes son la perforación y la hemorragia diferida (HD). La más grave es la primera, con incidencias que van desde el $0.15 \%$ al $1.5 \%$ según el tamaño de las lesiones resecadas ${ }^{47-49}$ y la más frecuente es el sangrado diferido ${ }^{44,45}$. La HD es la complicación potencialmente grave más frecuente de la RME, llevando en ocasiones a consecuencias clínicas no deseables y produciendo un notable consumo de recursos materiales.

\section{I.3.1 DEFINICIÓN}

La definición de hemorragia post-resección varía según los trabajos, aunque una de las más utilizadas en la siguiente: sangrado digestivo clínicamente evidente tras una resección endoscópica, manifestado como rectorragia o hematoquecia y que presenta al menos dos de los tres criterios siguientes: 
disminución del valor de hemoglobina en sangre $>2 \mathrm{~g} / \mathrm{dL}$, disminución de la presión arterial $>20 \mathrm{mmHg}$ y aumento de la frecuencia cardiaca $>20 \%$ de la basal ${ }^{44,45}$

La ausencia de criterios uniformes en la evaluación de los eventos adversos es probablemente una de las causas de la amplia variabilidad en la incidencia entre las distintas series.

\section{I.3.2 SUBTIPOS DE SANGRADO}

La hemorragia se clasifica en 2 subtipos, según su evolución temporal:

\section{- $\underline{\text { Hemorragia intraprocedimiento: }}$}

Aquella que ocurre durante el procedimiento ${ }^{45}$. Se considera clínicamente significativa si dura más de 30-60 segundos o requiere tratamiento endoscópico. En algunos estudios se ha establecido una graduación de la gravedad en función de datos endoscópicos objetivos y la duración de la misma (grado 1: autolimitado, 60 segundos; grado 2: sangrado babeante que cede en más de 60 segundos espontáneamente; grado 3: sangrado babeante que requiere tratamiento endoscópico; grado 4: sangrado arterial) ${ }^{45,70}$. La incidencia de la hemorragia intraprocedimiento oscila entre el $3.4 \%$ y el $24 \%$. Se ha asociado a un mayor tamaño de la lesión, a la morfología de tipo 0-lla+ls de la clasificación de Paris y a la histología de tipo velloso o tubulovelloso $44,45,71,72$.

\section{- $\underline{\text { Hemorragia diferida (HD): }}$}

La hemorragia diferida es aquella que sucede tras el alta de la unidad de endoscopia hasta los 15-30 días (Figura I.3). La incidencia de la HD es del $2.6 \%$ al 9.7\% según diversos trabajos ${ }^{44,70,81,82,73-80}$. Se han descrito como factores de 


\section{Introducción}

riesgo de HD post-resección la localización en colon proximal, el mayor tamaño de la lesión, la hemorragia intraprocedimiento, el uso de unidades electroquirúrgicas no controladas por microprocesador, la mayor edad del paciente, la hipertensión arterial, la toma de ácido acetil salicílico o anticoagulantes y la comorbilidad, en ocasiones medida por la clasificación anestésica ASA. Existen dos estudios muy interesantes que correlacionan hallazgos del defecto mucoso post-resección con el riesgo de $\mathrm{HD}^{83,84}$. En estos trabajos se ha visto una asociación positiva con el daño muscular, la aparición de hematomas submucosos o "cherry spots" y la presencia de vasos visibles. Además, el estudio de Kim et al diferencia entre vasos visibles intactos y vasos $\operatorname{cortados}^{84}$. Falta evidencia científica que correlacione el riesgo de HD con el conjunto de factores que podrían influir en dicha complicación: factores clínicos del paciente, características básicas del pólipo (tamaño y localización), y factores observados en la escara post-resección. El GSEED-RE está llevando a cabo un proyecto al respecto.

Un porcentaje importante de las HD ocurre dentro de las primeras 48 horas, por lo que algunos autores indican la observación en régimen de ingreso durante 24-48 horas de los pacientes de alto riesgo de sangrado o en aquellos con más difícil acceso al hospital. El 75\% ocurren en la primera semana postresección ${ }^{85}$.El manejo de los pacientes es generalmente ambulatorio, sin embargo, no hay claras recomendaciones establecidas al respecto ${ }^{86}$.

En la gran mayoría de las series, la mortalidad por esta complicación es prácticamente nula ${ }^{71}$. 
Figura I.3. Hemorragia diferida y tratamiento con hemoclips.
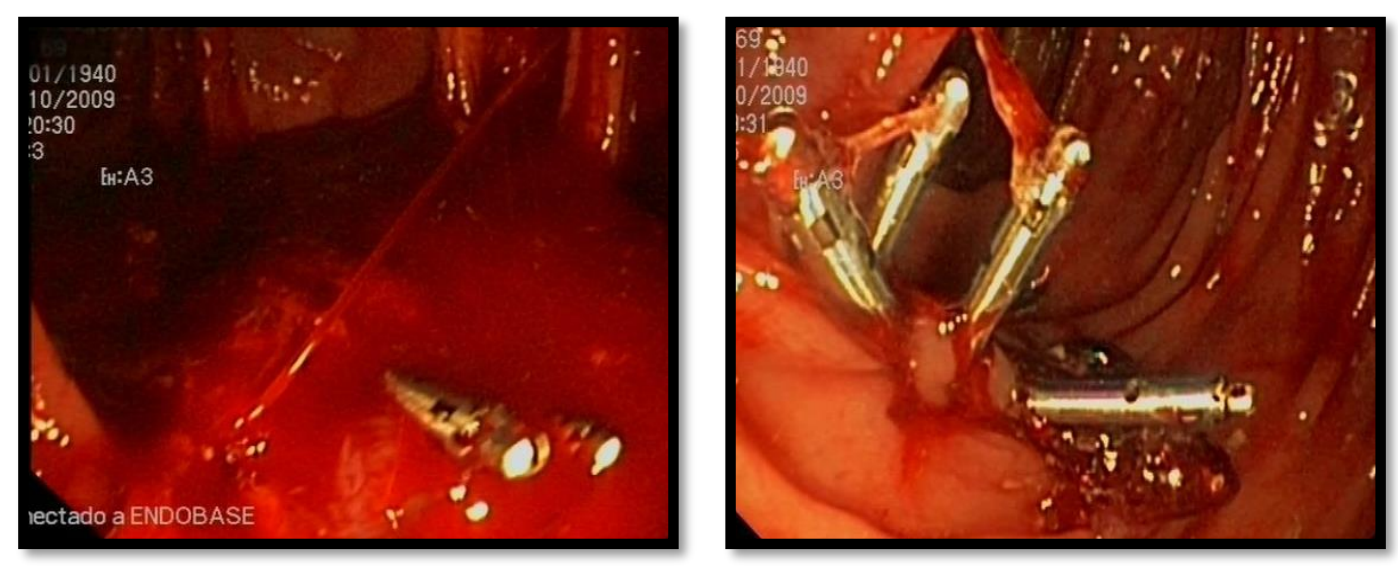

\section{I.3.3 MEDIDAS PREVENTIVAS}

\section{- Inyección submucosa}

La inyección de adrenalina en una dilución 1:10000 con suero fisiológico parece disminuir la incidencia de hemorragia intraprocedimiento, pero no la diferida. El grupo de trabajo australiano ha asociado el riesgo de sangrado diferido al intraprocedimiento aunque no se atribuye un efecto en el sangrado diferido en otros trabajos ${ }^{44}$. De hecho, es una tendencia actual no usar adrenalina en las soluciones de inyección con el fin de evitar la isquemia del tejido y facilitar la visualización de los vasos sangrantes durante el procedimiento con el fin de tratarlos en el propio acto endoscópico. Las nuevas soluciones de inyección submucosa que vienen precargadas no llevan adrenalina en su composición ${ }^{45,87-89}$. 


\section{Introducción}

Diversas soluciones con viscosidad mayor a las del suero salino, como la dextrosa 50\%, el ácido hialurónicoo el glicerol entre otros, han demostrado ser más efectivas que la solución salina para producir una elevación submucosa más prolongada, pero no para reducir el riesgo de complicaciones ${ }^{90-93}$.

\section{- Coagulación profiláctica de vasos visibles}

La coagulación preventiva de los vasos visibles con plasma de argón (APC) no ha demostrado reducir el riesgo de $\mathrm{HD}^{77}$.

Las pinzas de coagulación son útiles en el tratamiento de escaras de polipectomía que muestran un sangrado activo o vasos visibles tras una RME del tracto digestivo superior, pero este procedimiento no disminuía la incidencia de HD en lesiones colorrectales en un ensayo clínico randomizado ${ }^{81}$. En este trabajo la incidencia de sangrado diferido era el doble en el grupo control y los pacientes no fueron estratificados según riesgo hemorrágico. En otro estudio randomizado reciente el uso de las pinzas de coagulación redujo el sangrado menor o de pequeña cuantía, pero no el clínicamente significativo ${ }^{94}$.

La coagulación con la punta del asa de polipectomía ("snare soft tip coagulation") permite el control de los sangrados intraprocedimiento, aunque no existe evidencia suficiente de su papel en la prevención de la HD ${ }^{95}$.

\section{- Aplicación profiláctica de clips}

En series amplias como en el trabajo de Liquat et al ${ }^{81}$ el cierre de la escara con clips tras RME de lesiones $\geq 2 \mathrm{~cm}$ se asocia a menores tasas de HD (Figura I.4), lo cual también se había demostrado previamente en polipectomías en colon $^{85,96,97}$. Existen varios estudios aleatorizados y controlados en los que la 
colocación preventiva de clips no redujo significativamente el riesgo de HD aunque las lesiones registradas en dichos trabajos eran pequeñas ${ }^{98-101}$. Un metaanálisis de estos ensayos no mostró ningún beneficio del cierre preventivo. Esto puede explicarse debido a que el riesgo de HD en el grupo de control fue muy bajo (2.7\%), se incluyeron diversos tipos de técnicas (RME, DSE) y la mayoría de los pólipos analizados fueron de pequeño tamaño $(<2 \mathrm{~cm})^{102,103}$. Otro metaanálisis más reciente (Ayoub et al, 2019) sí que ha mostrado un efecto beneficioso significativo del cierre de los defectos mucosos con hemoclips, con una reducción del $5.2 \%$ de HD frente al $1.4 \%$ en el grupo de tratamiento (OR: $0.24,95 \% \mathrm{Cl}: 0.12-0.50, \mathrm{P}<0.001)^{104}$.

Tras estos trabajos han sido publicados dos ensayos clínicos aleatorizados que refuerzan el empleo de los hemoclips como medida profiláctica de la HD postresección. Ambos serán objeto de revisión posterior. Uno de ellos es uno de los trabajos principales de esta tesis, que se recoge en el artículo 3. Un metaánalisis con toda la nueva evidencia también será expuesto en el artículo 4.

Figura I.4. Cierre completo del defecto mucoso tras RME de LST-G.

A

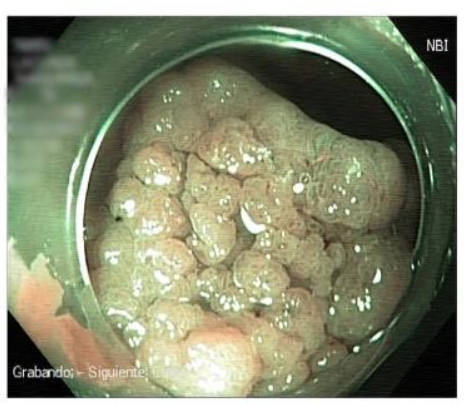

B

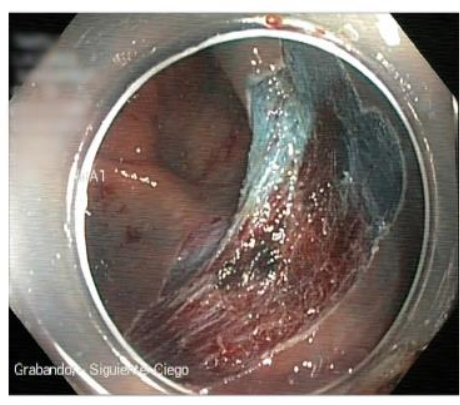

C

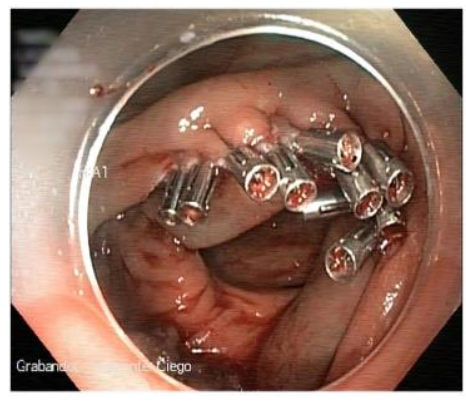

A) Adenoma granular mixto. B) Defecto mucoso tras RME. C) Cierre completo preventivo con_hemoclips. 


\section{Introducción}

\section{I.3.4 TRATAMIENTO DE LA HEMORRAGIA DIFERIDA}

A pesar de que los casos de HD pueden ser potencialmente graves, más de la mitad son autolimitados ${ }^{85,105,106}$. Tras la estabilización inicial del paciente, como en cualquier caso de hemorragia digestiva baja, la indicación de tratamiento endoscópico, embolización angiográfica o tratamiento quirúrgico se planteará de forma individualizada y según los recursos disponibles. En casos de hemorragia tras RME de lesiones grandes, un estudio del grupo australiano (Australian Colonic Endoscopic Resection Study-Group, ACER) demostró que la hematoquecia con una frecuencia mayor a 1 episodio/hora, la necesidad de transfusión y la clasificación ASA $\geq 2$ se han asociado a una mayor necesidad de tratamiento y la inestabilidad hemodinámica junto con los niveles de hemoglobina $<12 \mathrm{~g} / \mathrm{dL}$ se relacionaron con un peor pronóstico ${ }^{105}$. Otro estudio retrospectivo reciente ha mostrado que la hematoquecia continua o recurrente se asocia a mayor realización de colonoscopia con terapéutica endoscópica y que el resto de los pacientes en los que el sangrado cede espontáneamente y no recurre pueden ser dados de alta en 24 horas $^{106}$.

\section{- Tratamiento endoscópico}

La mayoría de las HD se consiguen cohibir con métodos endoscópicos: hemostasia por inyección de vasoconstrictores o esclerosantes (adrenalina, polidocanol), coagulación (punta de asa, sondas térmicas, pinza de coagulación o APC), diversos tipos de clips (introducidos a través del endoscopio (TTSC) o sistemas más complejos como los clips de gran tamaño premontados sobre capuchones (OTSC) o tratamientos combinados $2,75,79,85,96,97,104,105,107$. 
Los sprays hemostáticos se pueden utilizar para tratar hemorragias incoercibles por otros métodos, si bien su eficacia es transitoria ${ }^{108}$.

\section{- Radiología vascular intervencionista}

La embolización radiológica selectiva puede ser utilizada en casos de fracasos al tratamiento endoscópico y como alternativa al tratamiento quirúrgico, sin embargo es una técnica no exenta de complicaciones principalmente isquémicas ${ }^{46}$.

\section{- Tratamiento quirúrgico}

Un estudio multicéntrico japonés que incluyó 36.083 RMEs mostró una incidencia de hemorragia del 1.4\%; solo uno de los pacientes estudiados requirió tratamiento quirúrgico $(0.19 \%)$. En otras series, se precisó cirugía del $0.21 \%$ al 2.9\% por fracaso del tratamiento endoscópico ${ }^{31,36,46}$.

\section{- Nuevos tratamientos}

Desde hace ya algún tiempo se están utilizando diversos geles que se aplican en el defecto mucoso con el fin de prevenir la HD, incluso uno de ellos ya se encuentra comercializado. Sin embargo, existe poca evidencia al respecto y todavía no se han realizado estudios randomizados con placebo o comparativos con los hemoclips ${ }^{109,110}$. También se ha documentado anecdóticamente el uso de cianoacrilato como medida preventiva ${ }^{111}$. 


\section{I.4 JUSTIFICACIÓN DE LA INVESTIGACIÓN REALIZADA EN ESTA}

\section{TESIS}

La HD es la complicación mayor más frecuente de la RME y supone un notable gasto en recursos sanitarios y económicos. Se conocen algunos factores de riesgo implicados en la aparición de la HD tales como el tamaño de las lesiones y su localización en el colon derecho. Sin embargo, el papel de otros muchos factores como la hipertensión arterial, el uso de fármacos antiagregantes o anticoagulantes, la comorbilidad mayor o el riesgo anestésico no han sido corroborados de forma unánime en diferentes estudios clínicos. Faltan trabajos con amplio número de pacientes y guías clínicas que definan la mejor estrategia para prevenir esta complicación ${ }^{75-82}$.

En la actualidad existen dos modelos predictivos relativamente recientes que estiman el riesgo de HD en relación con diversos factores clínicos del paciente o morfológicos del pólipo. El primero de ellos (artículos 1 y 2 de esta tesis) fue desarrollado por el GSEED-RE y el segundo por el grupo ACER ${ }^{75}$. Aunque las dos series son amplias, incluyen variables que no coinciden y no han sido validadas por otros grupos de trabajo externos.

Hasta el año 2019, fecha en la cual se han escrito varios de los trabajos principales de esta tesis, no existían evidencias científicas sólidas que recomendaran realizar alguna medida profiláctica frente a la HD, a pesar de que esta complicación puede conllevar graves consecuencias clínicas.

Los hemoclips pueden liberarse sobre vasos puntuales o pueden emplearse para el cierre completo de los defectos mucosos secundarios a la RME. El cierre 
completo con clips parece ser un método muy efectivo, aunque este hecho sólo ha sido probado en un número escaso de estudios no controlados o en ensayos clínicos con gran heterogeneidad en cuanto al tipo de lesión tratada, a su tamaño y a la técnica utilizada96,97,102.

Como ejemplo, podemos citar el ensayo clínico de Zhang et $\mathrm{al}^{102}$, el cual mostraba que en LCRNP de 1 a $4 \mathrm{~cm}$ resecadas mediante RME o DSE el cierre completo de la escara mediante clips se asociaba a una menor tasa de síndrome post-polipectomía y HD y a una mayor satisfacción del paciente. No se pudo demostrar el coste-efectividad (CE) del mismo y los resultados de este estudio podrían estar influenciados por el uso de dos técnicas endoscópicas muy diferentes. Toda esta heterogeneidad se ha puesto de manifiesto en dos metaanálisis con resultados opuestos ${ }^{103,104}$.

Dada la escasa evidencia sólida y a su vez contradictoria, consideramos de gran interés aportar nuevos datos acerca de los factores de riesgo implicados en la aparición de HD post-RME y valorar si la aplicación de hemoclips preventivos es eficaz y coste-efectiva en la prevención de dicha hemorragia. 
upna 


\section{OBJETIVOS}

La presente tesis tiene los siguientes objetivos:

\section{O.1 OBJETIVOS PRINCIPALES}

Estimar el riesgo de HD tras RME de lesiones colorrectales de gran tamaño e identificarlos factores de riesgo implicados en su aparición (artículos 1 y 2).

Validar externamente los modelos predictivos de riesgo de HD propuestos en la literatura internacional en una cohorte multicéntrica española de pacientes (artículo 2).

Evaluar la eficacia mediante un ensayo clínico aleatorizado y un metaanálisis (artículos 3 y 4) y el coste-efectividad (artículo 5) del cierre completo de los defectos mucosos con hemoclips tras la RME de LCRNP de gran tamaño como método de prevención de la HD.

\section{O.2 OBJETIVOS SECUNDARIOS}

Realizar la calibración y adaptación de los modelos predictivos de riesgo de HD propuestos en la literatura al contexto del Sistema Nacional de Salud Español (artículo 2).

Desarrollar un modelo predictivo global a partir de los resultados de validación de los modelos predictivos internacionales aplicados a una cohorte multicéntrica española (artículo 2). 


\section{Objetivos}

Analizar la seguridad del cierre de defectos mucosos con hemoclips tras la RME de LCRNP de gran tamaño (artículo 3). 


\section{MÉTODOS}

\section{M.1 DISEÑO DEL ESTUDIO}

Para el desarrollo de los dos primeros objetivos principales se ha realizado un estudio observacional, multicéntrico, basado en una cohorte longitudinal recogida desde marzo de 2013 hasta noviembre de 2016 en diversos hospitales de España asociados al grupo de resección endoscópica de la GSEED-RE.

Para el tercer objetivo principal se ha diseñado un ensayo clínico controlado, aleatorizado, ciego simple, multicéntrico y nacional. El manejo clínico de los pacientes se ha adecuado a la práctica habitual, salvo en el grupo experimental, donde además se realiza el cierre de las escaras con clips. Este ensayo ha sido incluido en un metaanálisis con colaboraciones internacionales y posteriormente se ha realizado un estudio de Coste Efectividad (CE) sobre la aplicación de hemoclips con una visión económica global y que puede ser utilizado en muy diversos escenarios.

\section{M.2 INTERVENCIÓN /ALEATORIZACIÓN}

Los pacientes han sido sometidos a una RME o resección mediante endoscopia de una LCRNP, lo que supone una extirpación en un bloque o en fragmentos de una lesión superficial colorrectal plana o sésil mediante un asa de diatermia. Tras la resección queda una herida, escara o defecto mucoso en el 
colon o en el recto. En los pacientes del estudio observacional no se ha realizado ningún tipo de intervención diferente de las habituales.

En el ensayo clínico no se ha intervenido en las escaras en el grupo control salvo en situaciones de urgencia, mientras que en el grupo de tratamiento las escaras se han cerrado con hemoclips. La intervención ha consistido por tanto en el cierre completo con clips del defecto mucoso secundario a la realización de la RME. Se definió cierre completo, cuando los clips se aplicaron uno al lado del otro y no había áreas submucosas sustanciales en la línea de cierre.

En este ensayo, la aleatorización se ha realizado de forma automática por una secuencia computerizada visualizada en una base de datos on line diseñada al respecto.

\section{M.3 PLAN DE VISITAS}

A continuación, se describe el plan de visitas para el estudio observacional y para el ensayo clínico randomizado:

\section{- $\quad$ Visita 1}

Coincide con la visita que el paciente realiza ambulatoriamente o durante su ingreso hospitalario con el objetivo de informarle sobre la resección mucosa endoscópica.

- Evaluación de los criterios de inclusión y exclusión.

- Información acerca de la técnica de resección. 
- Información acerca de la participación en el estudio y acerca de la aleatorización en el caso del estudio. Entrega de consentimientos informados, de la RME y del estudio.

- Recogida de datos: datos demográficos y características basales.

\section{- $\quad$ Visita 2}

Día en el que se realiza la resección.

○ Recogida de consentimientos informados

- Aleatorización en el caso del ensayo: inclusión en el cuaderno de recogida de datos electrónico (CRDe) tras la RME. Cierre del defecto mucoso en los pacientes del grupo de tratamiento.

- Cumplimentación de la hoja de recogida de datos

- Las visitas 1 y 2 pueden coincidir en el tiempo.

\section{- $\quad$ Visita 3}

Consulta entre los 16 y 30 días posteriores a la resección, ya sea presencial o telefónica:

- Complicaciones tardías

- Aspectos relevantes de la hospitalización si la hubiera.

\section{- $\quad$ Visita 4}

Primer control endoscópico a los 3-6 meses. Recogida de los siguientes datos:

- Tratamiento completo o recurrencia de la lesión: datos endoscópicos e histológicos. 
Métodos

- $\quad$ Visita 5 (final)

Segundo control endoscópico a los 12 meses del primer control endoscópico.

Recogida de los siguientes datos:

- Tratamiento completo o recurrencia de la lesión: datos endoscópicos e histológicos. 


\section{M.4 CRITERIOS DE SELECCIÓN}

\section{M.4.1 CRITERIOS DE INCLUSIÓN}

Todos los pacientes deben cumplir los siguientes criterios de inclusión:

$\checkmark$ Adultos ( $\geq 18$ años) de ambos sexos.

$\checkmark$ Indicación clínica de resección mucosa endoscópica por LCRNP de gran tamaño ( $\geq 2 \mathrm{~cm})$.

$\checkmark$ Que otorguen su consentimiento informado por escrito tanto para la técnica de RME (según práctica habitual) como para el estudio observacional o el ensayo, en su caso.

Criterios adicionales para el ensayo clínico:

$\checkmark$ Pacientes con lesiones de riesgo medio o alto de HD (score de riesgo de HD, GSEED-RE $\geq 4$ ). No se contará para el score la variable de cierre con clips.

Los pacientes anticoagulados o en tratamiento con AINES de forma crónica se equipararán a los antiagregados en la puntuación del score: 2 puntos 
Métodos

\section{M.4.2 CRITERIOS DE EXCLUSIÓN}

Los pacientes que cumpliendo los criterios de inclusión tengan además alguna de las siguientes características serán excluidos del estudio:

× Lesiones resecadas mediante disección submucosa.

× Cualquier problema médico, psicológico, psiquiátrico, geográfico o social importante y no controlado que pueda interferir en la participación del paciente en el estudio o que no permitan un adecuado seguimiento $y$ adherencia con el protocolo y evaluación de los resultados del estudio.

En los pacientes del ensayo clínico se han tenido en cuenta los siguientes criterios adicionales de exclusión:

× Resecciones incompletas.

× Resecciones con sospecha de invasión en la submucosa profunda.

X Lesiones tratadas con clips por sospecha o certeza de daño en la muscular propia.

× Lesiones colorrectales con bajo riesgo de HD (Score de riesgo de HD < 3). 
Tabla M.1: Score de riesgo de HD GSEED-RE (Albéniz et al. Clin Gastroenterol Hepatol 2016).

\begin{tabular}{|l|c|}
\hline VARIABLE & SCORE \\
\hline Edad $\geq 75$ años & 1 \\
\hline Clasificación ASA III-IV & 1 \\
\hline Tamaño de la lesión $\geq 40 \mathrm{~mm}$ & 1 \\
\hline Tratamiento con antiagregantes, AINES o anticoagulantes & 2 \\
\hline $\begin{array}{l}\text { Defecto mucoso no cerrado con clips (no se utilizará este apartado } \\
\text { para el ensayo clínico) }\end{array}$ & 2 \\
\hline Localización proximal a colon transverso & 3 \\
\hline
\end{tabular}

Tabla M.2: Categorización de riesgo en pacientes según GSEED-RE.

\begin{tabular}{|l|l|}
\hline SCORE & RIESGO \\
\hline $0-3$ & Bajo $(0.6 \% ; 95 \% \mathrm{Cl} 0.2-1.8)$ \\
\hline $4-7$ & Medio $(5.5 \% ; 95 \% \mathrm{Cl} 3.8-7.9)$ \\
\hline $7-10$ & Alto $(40 \% ; 95 \% \mathrm{Cl} 21.8-61.1)$ \\
\hline
\end{tabular}




\section{M.5 VARIABLES E INSTRUMENTOS DE MEDICIÓN}

\section{M.5.1 CARACTERÍSTICAS BASALES}

- Datos demográficos: edad, sexo.

- Co-morbilidades mayores.

- Clasificación ASA.

- Medicación anticoagulante o antiagregante.

- Datos analíticos: hemoglobina, plaquetas, tiempo de protrombina, Índice internacional normalizado (INR).

\section{M.5.2 VARIABLE PRINCIPAL}

Hemorragia diferida: Aquella hemorragia digestiva baja evidente (hematoquecia o rectorragia) que ocurre desde el alta de la unidad de endoscopia hasta el día 15 post-RME y requiere cualquier tipo de asistencia médica.

\section{M.5.3 VARIABLES SECUNDARIAS}

- Relacionadas con la eficacia de la RME: resecciones completas, recurrencias en los diferentes controles endoscópicos.

- Relacionadas con las complicaciones: relacionadas con la hemorragia (secuencia temporal, gravedad, datos de hospitalización y tratamiento precisado entre otras). Incidencia de otras complicaciones derivadas de la RME: perforación, síndrome post-polipectomía (dolor, leucocitosis, 
febrícula-fiebre, irritación peritoneal sin perforación), fiebre, dolor abdominal post-RME, complicaciones derivadas de la anestesia, muerte.

- Relacionadas con el procedimiento: técnica empleada, tiempo procedimiento, marcaje de los límites de la lesión, tipo de corriente empleada, sustancias empleadas para la elevación, uso de APC, número de clips utilizados y propósito de su uso (hemostasia de vasos aislados o cierre completo), otros procedimientos hemostáticos, tipo de sedación.

- Características morfológicas de la lesión: tamaño, localización, clasificación LST, París, NICE, JNET, Kudo y Sano.

- Tecnología utilizada: tipo de endoscopio, NBI, cromoendoscopia convencional.

- Diagnóstico prepatológico realizado por el endoscopista: tipo de lesión, profundidad de la invasión, indicación de tratamiento endoscópico o quirúrgico.

- Días de ingreso necesarios tras la RME (derivados de la técnica y derivados de las complicaciones surgidas).

\section{M.6 ANÁLISIS ESTADÍSTICO}

Se ha efectuado una descripción de la muestra mediante la distribución de frecuencias para las variables categóricas, y mediante medidas de tendencia central y de dispersión (media y desviación estándar, mediana y rango intercuartílico) para las variables cuantitativas.

Para identificar factores de riesgo de DB se han utilizado modelos de regresión logística univariante y multivariante que han permitido evaluar 


\section{Métodos}

posibles interacciones entre los factores de riesgo, han proporcionado OR crudos y ajustados, con sus respectivos intervalos de confianza (IC) al $95 \%$.

Para validar los modelos predictivos del GSEED-RE y del grupo ACER, se ha utilizado una nueva de cohorte prospectiva de pacientes, y se ha valorado capacidad discriminativa y predictiva de cada modelo (y de sus versiones calibradas respectivas) mediante estadísticos como el área bajo la curva ROC y la pendiente de discriminación y su calibración mediante la prueba de bondad de ajuste de Hosmer-Lemeshow y los gráficos de calibración. A partir de los resultados de la validación se ha creado un modelo que proporciona los mejores valores de capacidad predictiva y estabilidad en las estimaciones, a la vez que integra la información más consistente del proceso de validación anterior.

En el ensayo clínico, se ha utilizado el análisis bivariante para el estudio de la asociación entre la intervención y la tasa de hemorragia tardía, así como de la relación entre las características de los pacientes, el tipo de lesión, y la incidencia de complicaciones. Para ello se han empleado el test Chi-cuadrado, el test de Fisher, el test de proporciones para modas y modelos de regresión logística univariantes.

Los métodos de nuestro metaanálisis y los criterios de inclusión de los estudios se basaron en las recomendaciones para revisiones sistemáticas y metaanálisis (PRISMA). El protocolo de revisión sistemática se registró en el Registro internacional prospectivo de revisiones sistemáticas (PROSPERO, www.crd.york.ac.uk/prospero/) en octubre de 2019. 
El análisis de CE se basó en tres fuentes: el estudio observacional prospectivo y multicéntrico que incluyó todas las RME de LCRNP registradas consecutivamente en la base de datos multicéntrica prospectiva del GSEED-RE (29 hospitales españoles) desde mayo de 2013 hasta julio de 2017 ( $n=2263$, trabajo 2 de la tesis), el metaanálisis resultante del trabajo 4 de la tesis y el ensayo clínico multicéntrico del trabajo 3 de la tesis que mostró el beneficio del cierre preventivo con clips en pólipos con riesgo sustancial de HD.

Los análisis estadísticos se realizaron mediante el programa estadístico IBM SPSS (v.20-25) (Inc. Chicago, IL) y el programa R 3.1.0 (R Foundation for Statistical Computing, Vienna, Austria. (http://www.R-project.org/).

\section{M.7 RECOGIDA DE DATOS}

Se ha planteó una recogida prospectiva de información, de manera que la obtención de datos se ha efectuado por medio de las visitas programadas según la práctica clínica habitual. Las fuentes de información han incluido la historia clínica del paciente y la información proporcionada por el facultativo.

Los pacientes incluidos en el estudio se han identificado mediante un código numérico. De este modo, la información recogida por cada investigador no contiene información personal que preserva la confidencialidad. A su vez, cada centro participante ha sido identificado en la base de datos del ensayo mediante un código. 
La aleatorización del ensayo se ha llevado a cabo mediante una secuencia computerizada. Se diseñó un CRDe disponible para todos los centros participantes en la web http://clips.biotektools.org/. Antes de comenzar el procedimiento de cierre con clips (RME ya realizada, valoración de criterios de inclusión y de exclusión), el endoscopista debía incluir al paciente en el CRDe y realizar el tratamiento asignado. De este modo, pacientes de diferentes centros han sido incluidos de una manera consecutiva en la secuencia aleatorizada.

El tratamiento, la comunicación y la cesión de los datos de carácter personal de todos los pacientes participantes se ha ajustado a lo dispuesto en la Ley Orgánica de Protección de Datos 3/2018 del 5 de Diciembre.

\section{M.8 ASPECTOS ÉTICOS}

Los estudios se han desarrollado de acuerdo con los principios éticos de la Declaración de Helsinki en su última revisión y con la legislación vigente.

El estudio observacional fue aprobado por el comité de ética de Navarra (ALBRME-2013-01) y registrado en clin trials.gov (NCT03050333).

El ensayo clínico fue aprobado por el comité de ética de Navarra (FMSALBE) y registrado en clin trials.gov (NCT02765022). 


\section{ARTÍCULO PRELIMINAR 1: Modelo predictivo del riesgo de hemorragia diferida tras la RME de lesiones colorrectales de gran tamaño.}

\section{A1.1 OBJETIVOS}

Los objetivos del trabajo fueron la identificación de factores de riesgo y de medidas preventivas asociadas a la HD post-resección de LCRNP de gran tamaño y el desarrollo de un modelo de predicción de riesgo d HD que pudiera sintetizarse en un score predictor de HD para estos pacientes.

\section{A1.2 PACIENTES Y MÉTODOS}

En 23 hospitales del GSEED de nuestro país se recogieron pacientes de forma prospectiva desde febrero del 2013 hasta febrero de 2015. Todos los pacientes con LCRNP de gran tamaño $(>2 \mathrm{~cm})$ con indicación de RME que cumplieron los criterios de inclusión citados previamente se incluyeron en nuestra base de datos. El Comité de Ética aprobó el protocolo del estudio (ALBRME-2013-01 / NCT03050333).

Se definió HD post-RME como aquel sangrado digestivo bajo evidente que requirió atención médica después del alta desde la unidad de endoscopia hasta el día 15 post-procedimiento.

Endoscopistas experimentados realizaron la RME asistida por inyección utilizando endoscopios de luz blanca convencionales o de alta definición. El 
tamaño de la lesión se midió con pinzas de biopsia abiertas o con el tamaño longitudinal de un asa de polipectomía abierta. Las escaras post-resección se cerraron a criterio del endoscopista. Se registró el tipo de corriente de electrocauterio utilizado (coagulación pura, corte puro o endocut u otras corrientes de mezcla) y el modelo de la fuente electroquirúrgica. Previo al procedimiento se confirmó un INR por debajo de 1,5 en los pacientes en tratamiento anticoagulante $y$ se suspendieron $o$ no los antiagregantes plaquetarios y se reiniciaron según las comorbilidades y las guías clínicas pertinentes. Todos los participantes fueron contactados por teléfono o tuvieron una entrevista personal a partir de los 15 días posteriores a la RME. El seguimiento se realizó en el servicio de consulta externa de cada centro e incluyó los controles endoscópicos de seguimiento.

En cuanto al análisis estadístico, las características de la muestra se describieron mediante estadística descriptiva. El riesgo de HD se estimó utilizando proporciones muestrales con IC del 95\%. Se ajustaron modelos de regresión logística univariante para identificar los factores de riesgo asociados con la HD. Incluimos las variables que eran significativas con nivel de significación $\alpha=0.1$ en un modelo de regresión multivariante y las usamos como factores predictivos finales si $p<0.1$. Se estimaron los ORs y el IC del $95 \%$ mediante análisis multivariante. La discriminación del modelo se determinó calculando el área bajo la curva de característica operativa del receptor (ROC) con su IC del 95\%. Evaluamos la calibración del modelo con la prueba de bondad de ajuste de Hosmer-Lemeshow, complementada con gráficos de calibración, utilizando el riesgo predicho contra el riesgo real en cada decil. Desarrollamos un score de 
riesgo asignando un peso a cada categoría de factor de riesgo basado en la estimación del parámetro $\beta$ de la regresión logística multivariante. A partir de la puntuación continua clasificamos a los pacientes en tres categorías de riesgo, bajo, medio y alto. El modelo fue validado internamente utilizando el método de validación interna de bootstraping para modelos logísticos con 150 muestras bootstrap.

\section{A1.3 RESULTADOS}

Se realizaron un total de 1255 RME de LCRNP de gran tamaño consecutivas en 1214 pacientes (ver Tabla A1.1). Cuarenta y seis pacientes tuvieron un episodio de HD [3,7\% (IC del 95\%: 2,7\%, 4,9\%)], dentro de las 48 horas en 17 pacientes (37\%) y entre 3-7 días en 23 (50\%). El riesgo de HD en pacientes con cierre completo de las escaras fue de $1,4 \%(4 / 281), 5,9 \%(11 / 185)$ cuando el cierre fue parcial (clips colocados para lograr la hemostasia de vasos aislados) y $3,9 \%(30 / 775)$ cuando no se utilizaron clips. Veinte pacientes $(43,5 \%)$ requirieron tratamiento endoscópico. La transfusión fue necesaria en 18 sujetos (39,1\%). Ningún paciente con HD requirió tratamiento quirúrgico y la mortalidad fue nula. La Tabla A1.2 resume los resultados del análisis univariante y la Tabla A1.3 los del multivariante. 
Tabla A1.1: Características descriptivas de la muestra (1255 RME en 1214 pacientes).

\begin{tabular}{|c|c|c|c|}
\hline Variable & $\mathbf{N}$ & Categorías & $\mathrm{n}(\%)$ \\
\hline \multicolumn{4}{|c|}{ Características demográficas } \\
\hline \multirow[t]{2}{*}{ Sexo } & 1214 & Femenino & $444(36.6 \%)$ \\
\hline & & Masculino & $770(63.4 \%)$ \\
\hline Edad (años) & 1214 & Media (DS) & $67.9(10.9)$ \\
\hline \multirow[t]{2}{*}{ Grupo de edad } & 1214 & $<75$ años & $843(69.4 \%)$ \\
\hline & & $\geq 75$ años & $371(30.6 \%)$ \\
\hline \multicolumn{4}{|l|}{ Características clínicas } \\
\hline \multirow[t]{4}{*}{ Clasificación ASA } & 1242 & 1 & $213(17.1 \%)$ \\
\hline & & ॥ & $650(52.3 \%)$ \\
\hline & & III & $345(27.8 \%)$ \\
\hline & & IV & $34(2.7 \%)$ \\
\hline \multirow[t]{3}{*}{ Aspirina } & 1242 & No & $1047(84.3 \%)$ \\
\hline & & Suspendida & $144(11.5 \%)$ \\
\hline & & Durante RME & $51(4.1 \%)$ \\
\hline Antiagregantes/ & 1241 & No & $917(73.9 \%)$ \\
\hline \multirow[t]{4}{*}{ anticoagulantes } & & Antiagregante & $209(16.8 \%)$ \\
\hline & & Dobleantiagregación & $9(0.7 \%)$ \\
\hline & & W/A & $100(8.1 \%)$ \\
\hline & & Dabigatran & $6(0.5 \%)$ \\
\hline \multirow[t]{6}{*}{ Clasificación de París } & 1248 & Ols/0ls+0lla & $547(43.9 \%)$ \\
\hline & & Olla & $559(44.8 \%)$ \\
\hline & & Ollb & $88(7.1 \%)$ \\
\hline & & Ollc & $10(0.8 \%)$ \\
\hline & & Olla+0llc & $38(3 \%)$ \\
\hline & & $011 b+0 \| l c$ & $6(0.5 \%)$ \\
\hline Tamaño_lesión (mm) & 1255 & Media (DS) & $30.5(11.8)$ \\
\hline Localización proximal a & 1253 & No & $610(48.7 \%)$ \\
\hline flexura esplénica & & $\mathrm{Si}$ & $643(51.3 \%)$ \\
\hline Localización proximal a & 1253 & No & $752(60.0 \%)$ \\
\hline flexura hepática & & $\mathrm{Si}$ & $501(40.0 \%)$ \\
\hline \multirow[t]{6}{*}{ Histología } & 1192 & Adenoma & $617(51.8 \%)$ \\
\hline & & DAG- Carc.-Im & $368(30.9 \%)$ \\
\hline & & Carc.-Invasivo & $80(6.7 \%)$ \\
\hline & & Serrado & $96(7.6 \%)$ \\
\hline & & DAG- Carc. Im serrado & $30(2.5 \%)$ \\
\hline & & Carc.-Invasivo en serrado & $1(0.1 \%)$ \\
\hline
\end{tabular}




\section{Características de la RME}

\begin{tabular}{|c|c|c|c|}
\hline \multirow[t]{2}{*}{ Resección } & 1236 & En fragmentos & $932(75.4 \%)$ \\
\hline & & En bloque & $304(24.6 \%)$ \\
\hline \multicolumn{4}{|l|}{ Adrenalina en injección } \\
\hline & 1247 & No & $406(32.6 \%)$ \\
\hline & & Si & $841(67.4 \%)$ \\
\hline \multirow[t]{3}{*}{ Corriente_electrocoagulación } & 1247 & Corte & 19(1.5\%) \\
\hline & & Coagulación & $172(13.8 \%)$ \\
\hline & & Mezcla (Endocut) & $1056(84.7 \%)$ \\
\hline \multirow[t]{2}{*}{ APC para_coagulación } & 1247 & No & 1163(93.3\%) \\
\hline & & $\mathrm{Si}$ & $84(6.7 \%)$ \\
\hline \multirow[t]{3}{*}{ Cierre con clips } & 1241 & No & $775(62.4 \%)$ \\
\hline & & Parcial & $185(14.9 \%)$ \\
\hline & & Completo & $281(22.6 \%)$ \\
\hline \multirow[t]{2}{*}{ Cierre_completo } & 1241 & No & $960(77.4 \%)$ \\
\hline & & $\mathrm{Si}$ & $281(22.6 \%)$ \\
\hline \multicolumn{4}{|l|}{ Eventos adversos } \\
\hline \multirow[t]{5}{*}{ Hemorragia: diferida } & 1247 & $\mathrm{Si}$ & $46(3.7 \%)$ \\
\hline & & $<24 \mathrm{~h}$ & $13(28.3 \%)$ \\
\hline & & $24-48 \mathrm{~h}$ & $4(8.7 \%)$ \\
\hline & & $3-7 d$ & $23(50 \%)$ \\
\hline & & $>7 \mathrm{~d}$ & $6(13 \%)$ \\
\hline Hemorragia & 1247 & $\mathrm{Si}$ & $66(5.3 \%)$ \\
\hline \multicolumn{4}{|l|}{ intraprocedimiento } \\
\hline \multirow[t]{3}{*}{ Perforación } & 1245 & No & $1228(98.6 \%)$ \\
\hline & & Si: solución endoscópica & $16(1.3 \%)$ \\
\hline & & Si: solución quirúrgica & $1(0.1 \%)$ \\
\hline Muerte & 1255 & No & $1255(100 \%)$ \\
\hline
\end{tabular}

APC: coagulación con plasma de argón. DAG: displasia alto grado; DS, desviación estándar; W/A: Warfarina o Acenocumarol; Carc. Im: Carcinoma intramucoso; Carc. Invasivo: Carcinoma invasivo. 
Tabla A1.2: Resultados de la regresión logística univariante para identificación de factores asociados a HD post-resección.

\begin{tabular}{|c|c|c|c|c|}
\hline Variable & Categoría & OR $(95 \% \mathrm{Cl})$ & $P$ & $\begin{array}{c}\mathbf{R}^{\mathbf{2}} \\
\text { Nagelkerke }\end{array}$ \\
\hline \multirow[t]{2}{*}{ Sexo } & Femenino & Referencia & & \\
\hline & Masculino & $1.30(0.68,2.47)$ & .425 & 0.002 \\
\hline \multirow[t]{2}{*}{ Grupo de edad } & $<75$ años & Referencia & & \\
\hline & $\geq 75$ años & $2.98(1.63,5.43)$ & $<.001$ & 0.038 \\
\hline \multirow[t]{2}{*}{ ASA } & $I-I \mid$ & Referencia & & \\
\hline & III-IV & $2.57(1.42,4.64)$ & .002 & 0.028 \\
\hline Aspirina & No & Referencia & & \\
\hline Durante RME & $\mathrm{Si}$ & $1.95(0.99,3.84)$ & .053 & 0.010 \\
\hline Antiagregantes/ & No & Referencia & & \\
\hline \multirow[t]{4}{*}{ anticoagulantes } & Antiagregante & $2.79(1.41,5.51)$ & & \\
\hline & $\begin{array}{l}\text { Dobleantiagre } \\
\text { gación }\end{array}$ & $12.87(2.46,67.2)$ & $<.001$ & 0.073 \\
\hline & W/A & $1.61(0.54,4.75)$ & & \\
\hline & Dabigatran & $38.6(7.39,201.6)$ & & \\
\hline \multirow[t]{2}{*}{ Clasificación París } & No Ols, Ols-0lla & Referencia & & \\
\hline & Ols, Ols-0lla, & $0.67(0.36,1.25)$ & .212 & 0.005 \\
\hline Proximal a & No & Referencia & & \\
\hline esplénico & Si & $3.55(1.74,7.21)$ & $<.001$ & 0.043 \\
\hline Proximal a & No & Referencia & & \\
\hline trasverso & Si & $4.49(2.30,8.76)$ & $<.001$ & 0.066 \\
\hline \multirow[t]{2}{*}{ Tamaño mm } & $<40$ & Referencia & & \\
\hline & $\geq 40$ & $1.81(0.97,3.37)$ & .061 & 0.010 \\
\hline \multirow[t]{2}{*}{ Histología } & Serrado & Referencia & & \\
\hline & Adenoma & $2.63(0.63,11.00)$ & .184 & 0.003 \\
\hline \multirow[t]{2}{*}{ Resección } & Fragmentos & Referencia & & \\
\hline & En bloque & $0.45(0.19,1.08)$ & .073 & 0.011 \\
\hline \multirow[t]{2}{*}{ Adrenalina } & No & Referencia & & \\
\hline & $\mathrm{Si}$ & $0.67(0.37,1.22)$ & .192 & 0.005 \\
\hline APC para & No & Referencia & & \\
\hline coagulación & $\mathrm{Si}$ & $0.73(0.26,2.01)$ & .564 & 0.001 \\
\hline \multirow[t]{3}{*}{ Cierre con clips } & No & Referencia & & \\
\hline & Parcial & $1.59(0.78,3.23)$ & .041 & 0.023 \\
\hline & Completo & $0.36(0.12,1.02)$ & & \\
\hline \multirow[t]{2}{*}{ Cierre completo } & $\mathrm{Si}$ & Referencia & & \\
\hline & No & $3.11(1.11,8.77)$ & .032 & 0.019 \\
\hline
\end{tabular}


W/A: Warfarina o Acenocumarol

Tabla A1.3: Resultados de regresión logística multivariante incluyendo predictores independientes de HD post-resección.

\begin{tabular}{|c|c|c|c|c|c|}
\hline Variable & Categorias & $\begin{array}{l}\text { OR ajustados } \\
\qquad(95 \% \mathrm{Cl})\end{array}$ & $P$ & $\beta$ & Score \\
\hline \multirow[t]{2}{*}{ Edad } & $<75$ años & Referencia & & & 0 \\
\hline & $\geq 75$ años & $2.36(1.24,4.51)$ & .009 & 0.860 & 1 \\
\hline \multirow[t]{2}{*}{ ASA } & $I-I \mid$ & Referencia & & & 0 \\
\hline & III-IV & $1.90(0.99,3.64)$ & .052 & 0.644 & 1 \\
\hline \multirow[t]{2}{*}{ Tamaño } & $<40 \mathrm{~mm}$ & Referencia & & & 0 \\
\hline & $\geq 40 \mathrm{~mm}$ & $1.91(0.99,3.70)$ & .055 & 0.647 & 1 \\
\hline \multirow[t]{2}{*}{ Aspirina } & No & Referencia & & & 0 \\
\hline & $\mathrm{Si}$ & $3.16(1.18,8.49)$ & .022 & 1.152 & 2 \\
\hline \multirow{2}{*}{$\begin{array}{l}\text { Cierre } \\
\text { completo }\end{array}$} & $\mathrm{Si}$ & Referencia & & & 0 \\
\hline & No & $3.63(1.25,10.53)$ & .018 & 1.288 & 2 \\
\hline \multirow{2}{*}{$\begin{array}{l}\text { Proximal a } \\
\text { trasverso }\end{array}$} & No & Referencia & & & 0 \\
\hline & $\mathrm{Si}$ & $4.86(2.43,9.71)$ & $<.001$ & 1.580 & 3 \\
\hline
\end{tabular}

ROC: 0.79 (95\%Cl 0.73-0.85)

Los predictores asociados significativamente con HD fueron la edad $\geq 75$ años (OR=2,36, IC 95\%: 1,24-4,51), el uso de aspirina durante la $R M E(O R=3,16$, IC 95\%: 1,18-8,49), la ubicación de la lesión proximal al colon transverso (OR=4,86, IC 95\%: 2,43-9,71) y no cerrar el defecto mucoso con hemoclips 
(OR=3,63, IC 95\%: 1,25-10,53). La clasificación ASA III o IV (OR=1,90; IC 95\%: 0,993,64) y el tamaño de la lesión $\geq 40$ mm (OR 1,91; IC 95\%: 0,99-3,70) alcanzaron un valor de $p$ de 0,05

Se diseñó un score con valores del 0 al 10 a partir de las estimaciones de los coeficientes del modelo de regresión multivariante (Tabla A1.3,A1.4 y A.1.5). El riesgo de HD asociado a cada valor del score se obtuvo de la formulación de la regresión logística utilizando los valores predichos. La muestra se clasificó en tres categorías de riesgo: bajo (score $0-3)$, medio (4-7) y alto (8-10).

Tabla A1.4: Probabilidad de HD en función del valor del score.

\begin{tabular}{|c|c|c|}
\hline Score & Riesgo de HD & Categoría de riesgo \\
\hline 0 & $0 \%$ & \multirow{4}{*}{$\begin{array}{c}\text { Baja } \\
n=618 \\
(0.6 \%)\end{array}$} \\
\hline 1 & $0 \%$ & \\
\hline 2 & $1 \%$ & \\
\hline 3 & $1 \%$ & \\
\hline 4 & $2 \%$ & \multirow{4}{*}{$\begin{array}{l}\text { Media } \\
n=541 \\
(5.5 \%)\end{array}$} \\
\hline 5 & $4 \%$ & \\
\hline 6 & $8 \%$ & \\
\hline 7 & $13 \%$ & \\
\hline 8 & $22 \%$ & \multirow{3}{*}{$\begin{array}{c}\text { Alta } \\
n=25 \\
(40 \%)\end{array}$} \\
\hline 9 & $34 \%$ & \\
\hline 10 & $48 \%$ & \\
\hline
\end{tabular}


Tabla A1.5: Valores de puntuación de los factores de riesgo de HD.

\begin{tabular}{|c|c|c|}
\hline \multicolumn{2}{|c|}{ Variable } & Score \\
\hline \multicolumn{2}{|c|}{ Edad $\geq 75$ años } & 1 \\
\hline \multicolumn{2}{|c|}{ Clasificación ASA III-IV } & 1 \\
\hline \multicolumn{2}{|c|}{ Tamaño lesión $\geq 40$ mm } & 1 \\
\hline \multicolumn{2}{|c|}{ Tratamiento con aspirina } & 2 \\
\hline \multicolumn{2}{|c|}{ No cierre completo con clips } & 2 \\
\hline \multicolumn{2}{|c|}{ Localización proximal a trasverso } & 3 \\
\hline \multicolumn{3}{|c|}{ Score total } \\
\hline $\begin{array}{c}\text { 0-3: Riesgo bajo } \\
(0.6 \% ; 95 \% \mathrm{Cl} 0.2-1.8)\end{array}$ & $\begin{array}{c}\text { 4-7:Riesgo medio } \\
\text { (5.5\%; 95\%Cl 3.8-7.9) }\end{array}$ & $\begin{array}{c}\text { 8-10: Alto riesgo } \\
(40 \% ; 95 \% \mathrm{Cl} 21.8-61.1)\end{array}$ \\
\hline
\end{tabular}

Esta distribución con tres categorías tuvo una $\mathrm{ROC}=0,77(95 \% \mathrm{Cl}$ 0,700,83 ) y la probabilidad de HD fue $0,6 \%$ (95\% Cl 0,2-1,8\%), 5,5\% (95\% Cl 3,8-7,9\%) y $40 \%$ (95\% IC 21,8-61,1\%), según los 3 tipos de riesgo (Tabla A1.5). El modelo multivariante tuvo una $\mathrm{ROC}=0,79(\mathrm{IC} 95 \% 0,73-0,85)$ y de manera similar, el score tuvo una ROC $=0,79$ (IC 95\% 0,72-0,85), mostrando una buena capacidad de discriminación. El valor estimado de ROC ajustado en el proceso de validación boostrasp fue de 0,73 (IC del 95\%: 0,66 a 0,79). La calibración del modelo también fue buena según las curvas de calibración (Figura A1.1) y los resultados de Hosmer-Lemeshow $(\mathrm{X} 2=5.72, \mathrm{P}=.572)$. La pendiente de discriminación (diferencia en las predicciones promedio para aquellos con y sin la variable resultado) fue de 0,07 , con un IC del $95 \%$ de 0,04 a 0,1 y $P<0,001$. 
Figura A1.1: Curva de calibración para el modelo de regresión multivariante

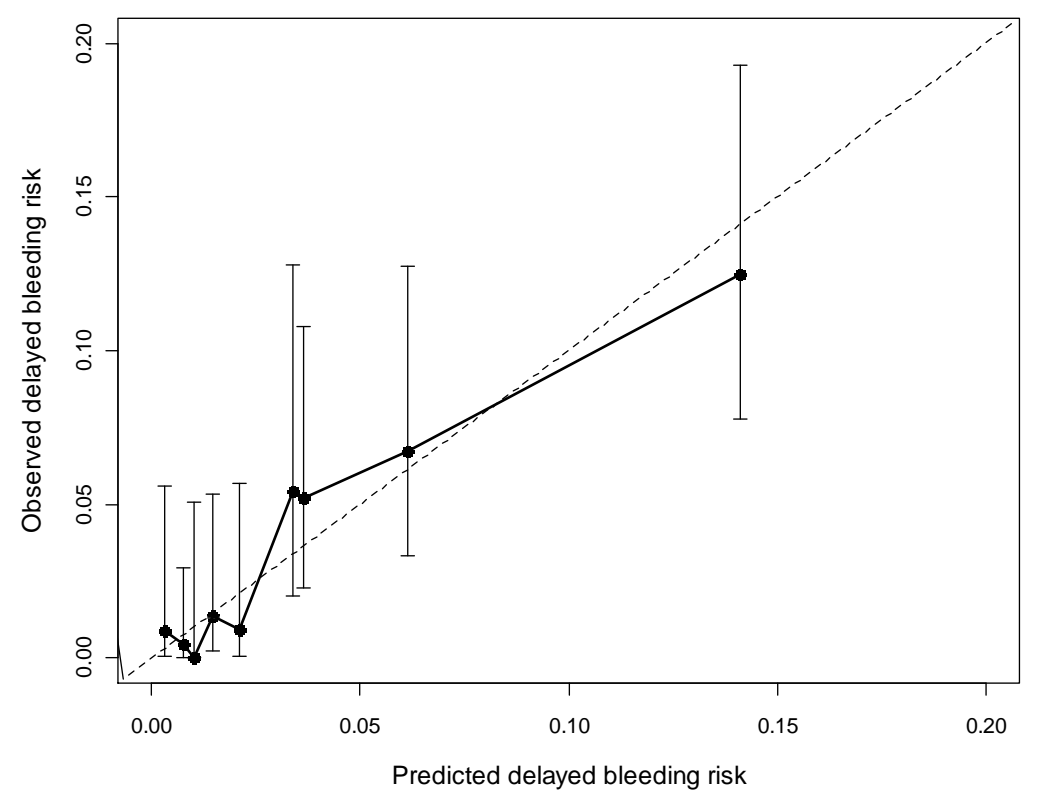

\section{A1.4 CONCLUSIONES}

En esta serie prospectiva, multicéntrica y con gran número de pacientes incluidos, seis variables se asociaron con HD después de la RME: edad $\geq 75$ años, clasificación ASA III o IV, uso de aspirina durante la RME, ubicación de la lesión proximal al colon transverso, tamaño de la lesión $\geq 40 \mathrm{~mm}$ y la ausencia de cierre completo de la escara con clips.

En nuestro trabajo el cierre preventivo de la escara con hemoclips redujo significativamente el riesgo de HD, sin embargo, otras medidas usadas teóricamente como prevención de hemorragia como el uso de adrenalina en la solución de inyección, la utilización preventiva de gas argón o de las pinzas de coagulación no tuvieron efectos significativos. 
Con estas variables desarrollamos un score predictivo para estimar el riesgo de HD post-resección que puede clasificarse en 3 categorías. Dos de las variables del score podrían considerarse modificables, como el uso de aspirina y el cierre con hemoclips de la escara, lo que otorga a este score la posibilidad de modificar a priori el riesgo de HD post-resección en estos pacientes. De esta manera proporcionamos la primera herramienta para identificar pacientes con alto riesgo de DB, que podría utilizarse para guiar el tratamiento profiláctico y el período de observación hospitalario. También podría ser útil en ensayos clínicos para evaluar estrategias de prevención (metodología empleada en nuestro ensayo clínico aleatorizado sobre el uso de hemoclips).

\section{A1.5 PUBLICACIÓN DEL TRABAJO}

Los resultados del estudio se publicaron inicialmente en forma de abstract en Gastrointestinal Endoscopy (GIE 2015; 81:5S, AB136) ${ }^{85}$ y se presentaron como comunicación oral en la Digestive Disease Week 2015 (Washington, USA) y en el congreso de la Sociedad Española de Endoscopia Digestiva donde obtuvo el premio Dr. Vázquez Iglesias a la mejor comunicación oral. La publicación final del artículo 1 (ver apéndice) fue en la revista Clinical Gastroenterology and Hepatology (FI 2019 8.549) $)^{112}$. 
upna 


\section{ARTíCULO 2. Validación clínica de los modelos predictores del riesgo de hemorragia diferida tras RME de lesiones colorrectales de gran tamaño.}

\section{A2.1 INTRODUCCIÓN Y OBJETIVOS}

En 2016 nuestro grupo de trabajo publicó el modelo predictivo de HD resumido en el apartado anterior ${ }^{112}$. También ese año, el grupo ACER desarrolló un score predictor de HD que utilizaba los siguientes factores de riesgo independientes: la ausencia de adrenalina en la solución de inyección, la comorbilidad mayor, el tamaño de la lesión y la localización proximal de las lesiones $^{113}$. Aunque ambas series incluyeron un gran número de pacientes, las variables resultantes de estos estudios no concordaban y ningún modelo había sido validado por otros grupos de investigación.

El objetivo principal de este segundo trabajo fue validar externamente los modelos predictivos de HD del GSEED-RE y del ACER. Los objetivos secundarios fueron actualizar o recalibrar estos modelos caso de ser necesario y desarrollar un nuevo modelo optimizado. 


\section{A2.2 PACIENTES Y MÉTODOS}

Veintiséis endoscopistas de 23 hospitales españoles del GSEED comenzaron un estudio observacional prospectivo multicéntrico en abril de 2013. Las LCRNP sometidas a RME se registraron consecutivamente en una base de datos centralizada. Los endoscopistas que participaron en el estudio realizaron un mínimo de 20 resecciones grandes por año. Todos los pacientes que tenían indicación de RME de lesiones colorrectales fueron seleccionados para su inclusión. El CEIC aprobó el protocolo del estudio (ALB-RME-2013-01 / NCT03050333).

La cohorte de pacientes incluidos en el primer modelo (GSEED-RE, Clin Gastroenterol Hepatol 2016, abril 2013-febrero 2015) constituyeron lo que denominamos en este capítulo cohorte pre-2015 y los pacientes incluidos entre marzo de 2015 y noviembre de 2016 constituyeron la cohorte de prueba o cohorte post-2015.

Se definieron de forma más precisa algunos aspectos técnicos. El defecto mucoso se cerró con hemoclips a criterio del endoscopista. El cierre de la escara se consideró parcial cuando no estaba completamente cerrado debido a su gran tamaño o ubicación compleja. El cierre puntual se definió como la colocación de clips en vasos aislados (vasos cortados, vasos con sangrado babeante). El manejo de anticoagulación y antiagregación se utilizó de manera uniforme siguiendo las guías disponibles. Los anticoagulantes orales tradicionales se retiraron 5 días antes del procedimiento y se estableció una terapia puente con heparina de bajo peso molecular hasta recuperar el nivel de anticoagulación esperado. Antes del 
procedimiento se confirmó un INR $<1,5$ en pacientes en tratamiento anticoagulante. La warfarina se reinició el día del procedimiento. Los anticoagulantes orales directos se retiraron 2-3 días antes del procedimiento y se reiniciaron al día siguiente. La aspirina no fue interrumpida en la mayoría de los casos, siendo cesada en pacientes de bajo riesgo trombótico. Las tienopiridinas se retiraron de forma rutinaria 5 días antes de la RME y se reintrodujeron el día después del procedimiento. Se contactó a los participantes 15-30 días después de la RME. El seguimiento se realizó de forma ambulatoria en cada centro.

En cuanto al análisis estadístico, las características de la cohorte post-2015 se presentaron como frecuencias (porcentajes) y medias (desviaciones estándar) y se compararon con las características de la cohorte pre-2015 mediante la prueba de chi-cuadrado, la prueba t de Student o la prueba de Fisher. El riesgo de HD se estimó utilizando proporciones muestrales con intervalos de confianza del 95\% y la evaluación de los predictores de HD se realizó mediante modelos de regresión logística. Utilizamos la cohorte post-2015 para validar el modelo GSEED-RE (M1a) y el modelo ACER original (M2a). La discriminación del modelo se comparó estimando el área bajo la curva ROC con IC del $95 \%$ y se complementó con la estimación de la pendiente de discriminación que midió la diferencia media en las probabilidades teóricas de HD entre pacientes con y sin HD observada. La calibración de los modelos se verificó con la prueba de bondad de ajuste de Hosmer-Lemeshow y con las gráficas de calibración de lo pronosticado contra el riesgo real en deciles. Además de los dos modelos originales, se evaluaron dos versiones actualizadas siguiendo las recomendaciones de la literatura para modelos de predicción de riesgos. Primero 


\section{Validación clínica del score de riesgo de hemorragia diferida}

aplicamos los modelos existentes a la cohorte de validación o cohorte post-2015 y recalibramos estos modelos cuando fue necesario. La primera actualización recalibró la versión original de cada modelo y nombró a los modelos Modelo 1b (M1b) y Modelo 2b (M2b) para el modelo GSEED-RE y el modelo ACER respectivamente. Los modelos revisados consistieron en el ajuste de la intersección y otros coeficientes de regresión del predictor por un factor de ajuste general (método 2 en Moons et al., 2012)114, lo cual es conveniente cuando la frecuencia de los resultados difiere entre el desarrollo y la muestra de prueba y/o los coeficientes de regresión del modelo original estaban sobreajustados o desajustados. La segunda actualización estimó los modelos M1c y M2c manteniendo las variables de los modelos originales pero reestimando los parámetros de dichos modelos, lo cual es conveniente cuando la fuerza de ciertos predictores puede ser diferente en la cohorte de validación

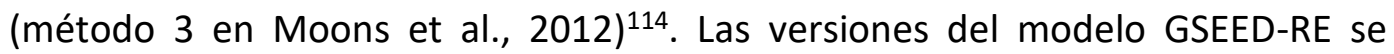
validaron además utilizando la cohorte española total (cohorte pre-2015 más cohorte post-2015), y los modelos ACER se validaron utilizando la cohorte española total y cohorte pre-2015. Finalmente se utilizó una regresión logística multivariante para crear un nuevo modelo (GSEED-RE2) que incluyó variables significativas en el análisis de regresión logística univariante para la cohorte post2015 y / o fueron incluidas en los modelos GSEED-RE y ACER. La variable uso de fármacos antiagregantes y anticoagulantes se incluyó en tres versiones. En el modelo M3a esta variable tenía 3 categorías: ninguno, antiagregantes y warfarina/acenocumarol/NOAC. En el modelo M3b tenía dos categorías: no o sí. El modelo en forma de score, M3c, se derivó de M3b. Este modelo M3c se ha 
denominado como GSEED-RE2. El rendimiento de los nueve modelos se evaluó y comparó utilizando la cohorte post-2015. La cohorte pre-2015 y la cohorte española total se utilizaron para validar M3a, M3b y M3c.

\section{A2.3 RESULTADOS}

En total se realizaron 1.034 RME de LCRNP de gran tamaño de forma consecutiva en 977 pacientes (62,1\% varones; edad media 68,1 años) (Tabla A2.1). Observamos una HD post-resección en 45 pacientes (4.5\%; IC del 95\% [3.3\% - 6.0\%]): en 17 pacientes (38\%) dentro de las 48 horas y en 18 pacientes (40\%) de 3 a 7 días después de la RME. Este riesgo de DB del 4,5\%, aunque superior, no presentó diferencias significativas con respecto al reviamente informada por nuestro grupo (3,7\%, IC del 95\% [2,7\% -4,9\%]).

Hubo algunas diferencias significativas con la cohorte pre-2015: se encontraron más lesiones proximales, menos pacientes con clasificación anestésica ASA III o IV y la tasa de uso de medicación antiagregante fue más baja (Tabla A2.1). En el análisis univariante, se identificaron las siguientes variables asociadas con HD: localización proximal (al ángulo esplénico o a flexura hepática), uso de antiagregantes, toma de anticoagulantes (tradicionales o NOAC) y dificultad subjetiva de la RME (ver apéndice, Artículo 2). 
Validación clínica del score de riesgo de hemorragia diferida

Tabla A2.1: Características descriptivas de la cohorte post-2015.

\begin{tabular}{|c|c|c|c|}
\hline Variable & $\mathbf{N}$ & Categorías & n (\%) \\
\hline \multicolumn{4}{|c|}{ Característicasdemográficas (n=977 pacientes) } \\
\hline \multirow[t]{2}{*}{ Sexo } & 975 & Femenino & $370(37.9 \%)$ \\
\hline & & Masculino & $605(62.1 \%)$ \\
\hline Edad (años) & 975 & Media (DS) & $68.1(10.4)$ \\
\hline \multirow[t]{2}{*}{ Grupo de edad } & 975 & $<75$ años & $689(70.7 \%)$ \\
\hline & & $\geq 75$ años & $286(29.3 \%)$ \\
\hline \multicolumn{4}{|c|}{ Característicasclínicas (n=1034 RME) } \\
\hline \multirow[t]{4}{*}{ Clasificación ASA } & 890 & 1 & $214(24.0 \%)$ \\
\hline & & ॥ & $460(51.7 \%)$ \\
\hline & & III & $201(22.6 \%)$ \\
\hline & & IV & $15(1.7 \%)$ \\
\hline \multirow[t]{2}{*}{ Comorbilidad } & 923 & No & $336(36.4 \%)$ \\
\hline & & $\mathrm{Si}$ & $587(63.6 \%)$ \\
\hline Antiagregantes/ & 951 & No & $703(68.0 \%)$ \\
\hline \multirow[t]{8}{*}{ Anticoagulantes } & & Aspirina suspendida & $83(8.0 \%)$ \\
\hline & & Aspirina durante RME & $43(4.2 \%)$ \\
\hline & & Clopidogrel & $14(1.4 \%)$ \\
\hline & & Aspirina + Clopidogrel & $9(0.9 \%)$ \\
\hline & & Otros antiagregantes & $11(1.1 \%)$ \\
\hline & & W/A & $76(7.4 \%)$ \\
\hline & & NOAC (Dabigatran) & $4(0.4 \%)$ \\
\hline & & $\begin{array}{l}\text { Antiagregantes y } \\
\text { Anticoagulantes }\end{array}$ & $8(0.8 \%)$ \\
\hline \multirow[t]{6}{*}{ Clasificación de Paris } & 1014 & Ols & $398(39,2 \%)$ \\
\hline & & Olla & $558(55,0 \%)$ \\
\hline & & Ollb & $29(2.9 \%)$ \\
\hline & & Ollc & $4(0.4 \%)$ \\
\hline & & Olla+Ollc & $24(2.4 \%)$ \\
\hline & & $0 l l b+0 \| l c$ & $1(0.1 \%)$ \\
\hline Tamaño lesión (mm) & 1031 & Media (DS) & $30.5(11.5)$ \\
\hline$<40 \mathrm{~mm}$ & & & $787(76.3 \%$ \\
\hline$\geq 40 \mathrm{~mm}$ & & & $244(23.7 \%)$ \\
\hline \multirow{2}{*}{$\begin{array}{l}\text { Localización proximal } \\
\text { al ángulo esplénico }\end{array}$} & 1034 & No & $387(37.4 \%)$ \\
\hline & & $\mathrm{Si}$ & $647(62.6 \%)$ \\
\hline \multirow{2}{*}{$\begin{array}{l}\text { Localización proximal } \\
\text { a flexura hepática }\end{array}$} & 1034 & No & $507(49.0 \%)$ \\
\hline & & $\mathrm{Si}$ & $527(51.0 \%)$ \\
\hline \multirow[t]{6}{*}{ Histología } & 999 & Adenoma & $525(52.6 \%)$ \\
\hline & & DAG-Carc. Im & $295(29.5 \%)$ \\
\hline & & Carc. invasivo & $49(4.9 \%)$ \\
\hline & & Serrado & $123(12.3 \%)$ \\
\hline & & DAG-Carc. Im en serrado & $6(0.6 \%)$ \\
\hline & & Carc. Invasivo en serrado & $1(0.1 \%)$ \\
\hline
\end{tabular}




\begin{tabular}{|c|c|c|c|}
\hline \multicolumn{4}{|c|}{ Características de la RME } \\
\hline \multirow[t]{2}{*}{ Resección } & 1018 & En fragmentos & $796(78.2 \%)$ \\
\hline & & En bloc & $222(21.8 \%)$ \\
\hline \multirow{2}{*}{$\begin{array}{l}\text { Adrenalina en } \\
\text { solución inyección }\end{array}$} & 973 & No & $319(30.9 \%)$ \\
\hline & & Si & $654(63.2 \%)$ \\
\hline \multirow[t]{3}{*}{ Corriente utilizada } & 972 & Corte & $2(0.2 \%)$ \\
\hline & & Coagulación & $103(10.6 \%)$ \\
\hline & & Mezcla (Endocut o similar) & $867(89.2 \%)$ \\
\hline \multirow[t]{2}{*}{ APC } & 1010 & No & $961(95.1 \%)$ \\
\hline & & Si & $49(4.7 \%)$ \\
\hline \multirow[t]{3}{*}{ Cierre con clips } & 972 & No & $562(57.8 \%)$ \\
\hline & & Parcial & $178(18.3 \%)$ \\
\hline & & Completo & $232(23.9 \%)$ \\
\hline \multirow[t]{2}{*}{ Cierre completo } & 972 & No & $740(76.1 \%)$ \\
\hline & & Si & 232(23.9\%) \\
\hline \multicolumn{4}{|l|}{ Eventos adversos } \\
\hline \multirow[t]{6}{*}{ Hemorragia diferida } & 1010 & No & $965(95.5 \%)$ \\
\hline & & Si & $45(4.5 \%)$ \\
\hline & & $<24 h$ & $8(0.8 \%)$ \\
\hline & & $24-48 h$ & $9(0.9 \%)$ \\
\hline & & $3-7 d$ & $18(1.7 \%)$ \\
\hline & & $>7 d$ & $10(1.0 \%)$ \\
\hline Hemorragia & 1008 & No & $951(94.2 \%)$ \\
\hline intraprocedimiento & & $\mathrm{Si}$ & $59(5.8 \%)$ \\
\hline \multirow[t]{2}{*}{ Perforación } & 1017 & No & 998(98.1\%) \\
\hline & & Si & 19(1.9\%) \\
\hline \multirow[t]{2}{*}{ Fallecimiento } & 1034 & No & $1034(100)$ \\
\hline & & $\mathrm{Si}$ & $0(100 \%)$ \\
\hline
\end{tabular}

DAG: displasia alto grado; RME: resección endoscópica mucosa; APC: coagulación con plasma de argón. W/A: Warfarina o Acenocumarol.

Validación de los modelos GSEED-RE y ACER utilizando la cohorte posterior a 2015.

Los modelos derivados del GSEED-RE se han denominado, M1a, M1b, M1c y los derivados del ACER, M2a, M2b, M2c. Sus especificaciones se muestran en el Apéndice, Artículo 2. La puntuación correspondiente a cada variable independiente según cada modelo predictivo se muestra en la Tabla A2.2. 
Tabla A2.2: Valores de puntuación utilizados en los scores M1 (GSEED-RE), M2 (ACER) y M3 (GSEEDE-RE2).

\begin{tabular}{|c|c|c|c|c|}
\hline Variable & Categoría & $\begin{array}{c}\text { M1 } \\
\text { (score 0-10) }\end{array}$ & $\begin{array}{c}\text { M2 } \\
\text { (score 0-6) }\end{array}$ & $\begin{array}{c}\text { M3 } \\
\text { (score 0-9) }\end{array}$ \\
\hline \multirow[t]{2}{*}{ Grupo de edad } & $<75$ años & 0 & - & - \\
\hline & >75 años & 1 & - & - \\
\hline Comorbilidad/ & No & 0 & 0 & 0 \\
\hline ASAIII-IV & $\mathrm{Si}$ & 1 & 1 & 1 \\
\hline \multirow[t]{4}{*}{ Tamaño (mm) } & $<30$ & - & 0 & - \\
\hline & $\geq 30$ & - & 2 & - \\
\hline & $<40$ & 0 & - & 0 \\
\hline & $\leq 40$ & 1 & - & 2 \\
\hline \multirow[t]{2}{*}{ Toma de aspirina } & No & 0 & - & - \\
\hline & $\mathrm{Si}$ & 2 & - & - \\
\hline Cierre completo con & $\mathrm{Si}$ & 0 & - & - \\
\hline clips & No & 2 & - & - \\
\hline Proximal flexura & No & 0 & 0 & 0 \\
\hline hepática & $\mathrm{Si}$ & 3 & 2 & 3 \\
\hline \multirow[t]{2}{*}{ Uso de epinefrina } & $\mathrm{Si}$ & - & 0 & - \\
\hline & No & - & 1 & - \\
\hline Antiagregante/ & No & - & - & 0 \\
\hline anticoagulante & $\mathrm{Si}$ & - & - & 3 \\
\hline
\end{tabular}

Cuando se aplicó el modelo GSEED-RE a la cohorte post-2015 ninguno de los modelos alcanzó un área bajo la curva $(A \cup C)$ de 0,70 a pesar de una buena calibración y mejora en M1b (modelo recalibrado) y M1c (modelo reestimado) (Tabla A2.3). Los porcentajes de pacientes con HD en las categorías de riesgo bajo, medio y alto fueron del 3,5\%, 5,9\% y $10,5 \%$, respectivamente (tabla A2.4), lo que sugiere un gradiente consistente aunque menos marcado que la cohorte pre-2015 (0,6\%, 5,5\% y 40\%, respectivamente). 
Tabla A2.3: Resumen del rendimiento de los modelos para la cohorte post-2015, cohorte pre-2015 y la cohorte total.

\begin{tabular}{|c|c|c|c|}
\hline $\begin{array}{l}\text { Indicadores } \\
\text { de rendimiento }\end{array}$ & $\begin{array}{l}\text { Post-2015 } \\
\text { (n=977 RME) }\end{array}$ & $\begin{array}{l}\text { Pre-2015 } \\
(n=1255)\end{array}$ & $\begin{array}{l}\text { Cohorte total } \\
\quad(n=2289)\end{array}$ \\
\hline \multicolumn{4}{|l|}{ Modelo GSEED-RE } \\
\hline \multicolumn{4}{|l|}{$\overline{\text { M1a - Score original }}$} \\
\hline ROC (IC 95\%) & $0.62(0.52,0.71)$ & $0.79(0.72,0.85)$ & $0.71(0.65,0.77)$ \\
\hline Hosmer-Lemeshow & $x^{2}=37.19 ; p<0.001$ & $5.72 ; p=0.572$ & $13.7 ; p=0.089$ \\
\hline Pte. discriminación & $0.019, p=0.036$ & $0.068 ; p<0.001$ & $0.045 ; p<0.001$ \\
\hline \multicolumn{4}{|c|}{ M1b - Modelo recalibrado } \\
\hline ROC (IC 95\%) & $0.62(0.52,0.71)$ & & $0.71(0.65,0.77)$ \\
\hline Hosmer-Lemeshow & $X^{2}=5.34 ; p=0.376$ & Recalibración & $3.09 ; p=0.686$ \\
\hline Pte. discriminación & $0.008 ; p=0.022$ & No necesaria & $0.032 ; p<0.001$ \\
\hline \multicolumn{4}{|c|}{ M1c - Modeloreestimado } \\
\hline ROC (IC 95\%) & $0.64(0.54,0.74)$ & & $0.71(0.65,0.77)$ \\
\hline Hosmer-Lemeshow & $X^{2}=10.77, p=0.215$ & Recalibración & $1.77 ; p=0.972$ \\
\hline Pte. discriminación & $0.016 ; P=0.003$ & No necesaria & $0.034 ; p<0.001$ \\
\hline \multicolumn{4}{|l|}{ ACER model } \\
\hline \multicolumn{4}{|l|}{$\overline{\mathrm{M} 2 \mathrm{a}-\mathrm{Score}}$ original } \\
\hline ROC (IC 95\%) & $0.61(0.52,0.71)$ & $0.69(0.62,0.76)$ & $0.66(0.60,0.71)$ \\
\hline Hosmer-Lemeshow & $X^{2}=23.76 ; p=0.002$ & $33.2 ; p<0.005$ & $54.00 ; p<0.001$ \\
\hline Pte. discriminación & $0.027 ; p=0.014$ & $0.036 ; p<0.001$ & $0.033 ; p<0.001$ \\
\hline \multicolumn{4}{|c|}{ M2b - Modelo recalibrado } \\
\hline ROC (IC 95\%) & $0.63(0.54,0.72)$ & $0.69(0.62,0.76)$ & $0.66(0.60,0.72)$ \\
\hline Hosmer-Lemeshow & $X^{2}=3.26, p=0.515$ & $3.30 ; p=0.508$ & $1.51 ; P=0.825$ \\
\hline Pte. discriminación & $0.011 ; P=0.010$ & $0.021 ; p<0.001$ & $0.016 ; p<0.001$ \\
\hline \multicolumn{4}{|c|}{ M2c - Modeloreestimado } \\
\hline ROC (IC 95\%) & $0.65(0.57,0.73)$ & $0.73(0.67,0.80)$ & $0.69(0.64,0.74)$ \\
\hline Hosmer-Lemeshow & $X^{2}=5.7, p=0.680$ & $8.86 ; P=0.263$ & $6.71 ; P=0.568$ \\
\hline Pte. discriminación & $0.012 ; P=0.003$ & $0.024 ; p<0.001$ & $0.018 ; P<0.001$ \\
\hline \multicolumn{4}{|l|}{ Nuevo modelo } \\
\hline \multicolumn{4}{|c|}{$\overline{\text { M3a - Antiagregantes/anticoagulantes en } 3 \text { categorías }}$} \\
\hline ROC (IC 95\%) & $0.69(0.60,0.77)$ & $0.74(0.67,0.80)$ & $0.71(0.65,0.77)$ \\
\hline Hosmer-Lemeshow & $X^{2}=9.92 ; p=0.193$ & $7.84 ; p=0.250$ & $4.53 ; p=0.605$ \\
\hline Pte. discriminación & $0.037 ; p<0.001$ & $0.039 ; p<0.001$ & $0.036 ; p<0.001$ \\
\hline \multicolumn{4}{|c|}{ M3b - Antiagregantes/anticoagulantes en 2 categorías } \\
\hline ROC (IC 95\%) & $0.69(0.59,0.78)$ & $0.73(0.67,0.80)$ & $0.71(0.65,0.77)$ \\
\hline Hosmer-Lemeshow & $x^{2}=11.9 ; p=0.102$ & $7.85 ; P=0.250$ & $4.73 ; p=0.579$ \\
\hline Pte. discriminación & $0.034 ; p<0.001$ & $0.039 ; p<0.001$ & $0.035 ; p<0.001$ \\
\hline \multicolumn{4}{|l|}{ M3c } \\
\hline ROC (IC 95\%) & $0.69(0.59,0.78)$ & $0.73(0.66,0.80)$ & $0.71(0.65,0.77)$ \\
\hline Hosmer-Lemeshow & $x^{2}=11.8 ; p=0.037$ & $6.53 ; p=0.588$ & $6.81 ; p=0.235$ \\
\hline Pte. discriminación & $0.033 ; p<0.001$ & $0.037 ; p<0.001$ & $0.035 ; p<0.001$ \\
\hline
\end{tabular}


Tabla A2.4: Probabilidad de HD en las categorías de riesgo bajo, medio y alto para las diferentes puntuaciones en la cohorte post-2015, pre-2015 y total.

\begin{tabular}{|c|c|c|c|c|c|c|}
\hline & \multicolumn{2}{|c|}{$\begin{array}{c}\text { M1 } \\
\text { (score 0-10) }\end{array}$} & \multicolumn{2}{|c|}{$\begin{array}{c}\text { M2 } \\
\text { (score 0-6) }\end{array}$} & \multicolumn{2}{|c|}{$\begin{array}{c}\text { M3 } \\
\text { (score 0-9) }\end{array}$} \\
\hline & Score & Prob.HD & Score & Prob.HD & Score & Prob.HD \\
\hline \multicolumn{7}{|c|}{ Cohorte Post-2015 } \\
\hline Riesgo bajo & $0-3$ & $3.5 \%$ & $0-1$ & $3.3 \%$ & $0-3$ & $2.2 \%$ \\
\hline Riesgo medio & $4-7$ & $5.9 \%$ & $2-4$ & $4.0 \%$ & $4-6$ & $4.9 \%$ \\
\hline Riesgo alto & $8-10$ & $10.5 \%$ & $5-6$ & $8.9 \%$ & $7-9$ & $17.1 \%$ \\
\hline \multicolumn{7}{|c|}{ Cohorte Pre-2015 } \\
\hline Riesgo bajo & $0-3$ & $0.6 \%$ & $0-1$ & $0.4 \%$ & $0-3$ & $2.1 \%$ \\
\hline Riesgo medio & $4-7$ & $5.5 \%$ & $2-4$ & $3.6 \%$ & $4-6$ & $3.4 \%$ \\
\hline Riesgo alto & 8-10 & $40 \%$ & $5-6$ & $10.0 \%$ & $7-9$ & $13.4 \%$ \\
\hline \multicolumn{7}{|l|}{ Cohorte total } \\
\hline Riesgo bajo & $0-3$ & $1.7 \%$ & $0-1$ & $1.4 \%$ & $0-3$ & $2.2 \%$ \\
\hline Riesgo medio & $4-7$ & $5.7 \%$ & $2-4$ & $3.4 \%$ & $4-6$ & $4.1 \%$ \\
\hline Riesgo alto & 8-10 & $27.3 \%$ & $5-6$ & $9.8 \%$ & $7-9$ & $14.8 \%$ \\
\hline
\end{tabular}

Al igual que los modelos basados en GSEED-RE ninguno de los modelos basados en ACER alcanzó la AUC mínima de 0,70, aunque la calibración fue suficiente en M2b (modelo recalibrado) y M2c (modelo reestimado) (Tabla A2.3). Los porcentajes de pacientes con HD en las categorías de riesgo bajo, medio y alto fueron del 3,3\%, 4,0\% y $8,9 \%$ respectivamente (Tabla A2.4), lo que sugiere un gradiente consistente.

Las covariables propuestas en el nuevo modelo (M3a) incluyeron comorbilidad, tamaño $\geq 40 \mathrm{~mm}$, localización proximal al ángulo hepático y uso de antiagregantes /anticoagulantes en tres categorías (puntuación de 0 a 9 puntos; 
Tabla A2.2). El AUC resultante fue 0,69 (IC del 95\% [0,60-0,77]) con una pendiente de discriminación de 0,037 ( $p<0,001)$. Este modelo mostró una capacidad de discriminación razonable y una prueba de Hosmer-Lemeshow no significativa con una gráfica de calibración aceptable (Tabla A2.3). Los resultados obtenidos con M3b presentaron una ROC, una pendiente de discriminación y una calibración similares (Tabla A2.3). M3c (GSEED-RE2) es una versión simplificada de M3b. Este modelo mostró una AUC de 0,69 (IC del 95\% $[0,59,0,78]$ ) y una pendiente de discriminación de 0,033 ( $p<0,001)$. La prueba de HosmerLemeshow no mostró una buena calibración $(p=0.037)$ aunque las gráficas de calibración fueron aceptables. Por lo tanto, el GSEED-RE2 proporcionó resultados de discriminación ligeramente mejores que los modelos GSEED-RE y ACER con una curva AUC cercana a 0,7, una pendiente de discriminación que duplicó su magnitud y gráficos de calibración razonables. Los porcentajes de pacientes con HD en las categorías de riesgo bajo, medio y alto fueron del $2,2 \%, 4,9 \%$ y $17,1 \%$, respectivamente, lo que sugiere un gradiente constante que fue más marcado que los modelos GSEED-RE y ACER.

\section{Validación del nuevo modelo utilizando las cohortes españolas pre-2015 y total}

El rendimiento del modelo se evaluó utilizando la cohorte pre-2015 y mostró buenos resultados para M3 en sus tres versiones. Por ejemplo, el M3c simplificado proporcionó una AUC de 0,73 (IC del 95\% $[0,66,0,80]$ ), una pendiente de discriminación de 0,037 $(p<0,001)$ y resultados de calibración aceptables. La HD ocurrió en 91 pacientes en la cohorte española total (cohorte pre-2015 más cohorte post-2015, $\mathrm{n}=2289 \mathrm{RME}$ ) (Estimación del riesgo de 
hemorragia en la cohorte total del $4 \%$, IC del $95 \%[3,3 \%-4,9 \%])$. El rendimiento de los modelos en la cohorte total fue aceptable, con todas las versiones M3 exhibiendo una AUC superior a 0,70 en todos los casos (ver Tabla A2.3). En la Figura A2.1 se muestra un diagrama resumen derivado de la cohorte total, que indica los pesos asignados a cada variable y el riesgo asociado con cada valor de puntuación.

Figura A2.1. Nuevo Score de riesgo de hemorragia diferida para la RME de LCRNP de gran tamaño: GSEED-RE2

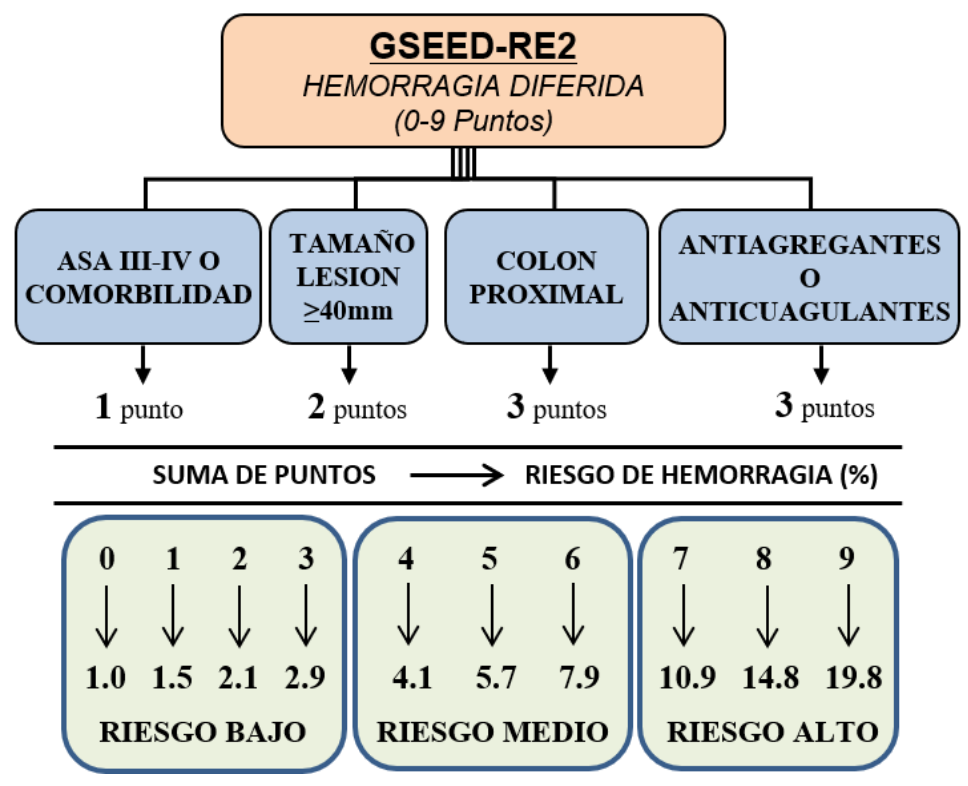

\section{A2.4 CONCLUSIONES}

La validación de los modelos GSEED-RE y ACER mostró resultados subóptimos. Este estudio supone la primera validación externa del modelo ACER.

Se ha desarrollado un nuevo modelo denominado GSEED-RE2 que predice la aparición de HD post-resección con una capacidad predictiva razonable. Este 
modelo elimina ciertas variables que no eran consistentemente significativas en las diferentes cohortes (cierre del defecto mucoso, edad y uso de adrenalina en la solución de inyección) e incluye cuatro variables fácilmente identificables: comorbilidad mayor, tamaño mayor de $4 \mathrm{~cm}$, lesiones localizadas proximales al ángulo hepático y uso de antiagregantes o anticoagulantes. Estas variables pueden identificarse con facilidad antes del procedimiento y constituyen una herramienta que ayuda al clínico a clasificar a los pacientes por su riesgo de HD y a diseñar "a priori" la mejor estrategia profiláctica según dicho riesgo.

EI GSEED-RE2 presenta unas curvas ROC aceptables en todas las cohortes, unos gráficos de calibración más estables, con una pendiente de discriminación mayor y más consistente que permite una mejor predicción del riesgo de HD.

La principal utilidad del modelo es la identificación de los pacientes con alto riesgo de HD, que suponen solamente el $10 \%$ del total. De esta manera se pueden seleccionar aquellos pacientes que pueden beneficiarse más de las medidas preventivas como el cierre de los defectos mucosos o un manejo más personalizado de la antiagregación o anticoagulación (los modelos previos no incluían la toma de anticoagulantes). El score también puede ser útil en cuestiones logísticas como la necesidad de apoyo familiar o de permanecer cerca del hospital al menos durante la primera semana en los pacientes de alto riesgo. Finalmente, el nuevo modelo puede mejorar la selección de candidatos para su inclusión en ensayos clínicos, lo que asegura datos más fiables y reduce el tamaño muestral con el consiguiente notable ahorro de recursos. 


\section{A2.5 PUBLICACIÓN DEL TRABAJO}

Los resultados del estudio se publicaron inicialmente en forma de abstract en la revista Endoscopy (Endoscopy 2017; 49(11): 1129-1194). La publicación final del artículo 2 (ver apéndice) ha sido en la revista Gastrointestinal Endoscopy (FI 2019: 6.890) $)^{115}$. 


\section{ARTíCULO 3. Cierre completo con hemoclips tras RME de lesiones colorrectales con riesgo sustancial de sangrado. Ensayo clínico aleatorizado.}

\section{A3.1 INTRODUCCIÓN Y OBJETIVOS}

Como se ha comentado en la introducción general, la evidencia disponible acerca de la utilidad del cierre de las escaras post-resección como medida preventiva de HD es contradictoria. Esto se debe a que los estudios han incluido pólipos de diversa naturaleza (pediculados y no pediculados), la mayoría de las lesiones tenían pequeño tamaño y por tanto, bajo riesgo de HD e incluso se han utilizado técnicas de tratamiento diferentes en algunos estudios (RME, RME híbrida y DSE) sin estimar su implicación en la variable resultado. A pesar de todo, la utilización preventiva de los hemoclips es una maniobra rutinaria entre endoscopistas de todo el mundo y no se ajusta a criterios de riesgo de sangrado. Cabe destacar que el gasto en clips es una de las partidas económicas más importantes de las unidades de endoscopia.

En este estudio controlado aleatorizado, nuestro objetivo fue valorar la eficacia del cierre profiláctico completo de los defectos mucosos post-RME de LCRNP para prevenir la HD en casos con un riesgo sustancial de presentar dicha complicación. 


\section{A3.2 PACIENTES Y MÉTODOS}

Este estudio paralelo aleatorizado controlado fue realizado en 11 hospitales españoles por 11 endoscopistas experimentados del GSEED-RE.

El protocolo del estudio cumplió con las guías éticas de la Declaración de Helsinki, recibió la aprobación del Comité de Ética en cada centro participante (código local: FMSALBE) y se registró en ClinicalTrials.gov (NCT02765022). Se obtuvo el consentimiento informado por escrito de todos los pacientes incluidos.

Los criterios de inclusión fueron adultos consecutivos mayores de 18 años remitidos y programados para RME de una LCRNP con riesgo sustancial de HD (puntuación GSEED-RE $\geq 6$, esto es, $\geq 8 \%$ de riesgo de HD), ya que se asumió que la variable de "no cierre con clips" estaba inicialmente presente en todos los casos. Se incluyeron en el estudio pacientes con una sola LCRNP. Los criterios de exclusión ya fueron comentados en la introducción general.

Los aspectos técnicos de las RME no difirieron de los trabajos previos. Se siguieron las guías ESGE de manejo de anticoagulación ${ }^{2}$. Se evitó el uso de adrenalina diluida en la solución de inyección y no se realizaron maniobras hemostáticas salvo que fuera necesario, quedando debidamente documentadas.

Los participantes fueron asignados al azar al grupo de cierre con clips o no cierre, mediante una secuencia computarizada con una tasa de asignación de 1: 1. La asignación de aleatorización se mostró automáticamente en nuestro programa protegido con contraseña basado en la web, http://clips.biotektools.org. Se intentó el cierre completo de la escara hemoclips de $11 \mathrm{~mm}$ (Micro-Tech ${ }^{\circledR}$, Nanjing, China) en todos los pacientes asignados al 
grupo de clips. Dentro del grupo de tratamiento definimos 3 subgrupos: cierre completo cuando los clips se aplicaron uno al lado del otro y no había áreas submucosas sustanciales en la línea de cierre; cierre parcial cuando sólo se pudo cerrar una parte del defecto mucoso; y cierre fallido cuando no se pudo cerrar ninguna parte de la escara o el endoscopista consideró que el defecto mucoso no se podía cerrar por su gran tamaño o difícil localización.

La variable resultado principal fue el riesgo de HD post-resección que se definió como hematoquecia clínicamente evidente que requirió atención médica (el paciente tuvo que comunicarse con un centro sanitario para una evaluación de su sangrado y requirió hospitalización, transfusión de sangre, cirugía, angiografía o repetición de colonoscopia) dentro de los 15 días posteriores al procedimiento. Las variables resultado secundarias fueron otros eventos adversos: sangrado inmediato, síndrome post-polipectomía, fiebre y dolor.

En cuanto al análisis estadístico, se calculó que era necesario un tamaño de muestra inicial de 332 pacientes para identificar como significativa una reducción del riesgo de $\mathrm{HD}$ del $8 \%$ al $2 \%$ en una prueba bilateral asumiendo un valor $\alpha$ del $5 \%$, un poder estadístico del $80 \%$ y una tasa de pérdidas del $2 \%$. Sin embargo, se consideraron otros posibles tamaños de muestra dependiendo del riesgo de HD observadas en el grupo de control. Después de que algunos investigadores expresaron su preocupación por las altas tasas de HD en sus cohortes un comité interno de seguridad de ensayos clínicos realizó un análisis provisional no planificado cuando se habían reclutado 200 pacientes. El investigador principal estaba cegado a los resultados. Se recalculó un nuevo tamaño de muestra de 227 participantes para identificar como significativa una reducción del riesgo de HD 
del $10 \%$ al $2 \%$ en una prueba bilateral asumiendo un poder estadístico del $80 \%$ y un valor $\alpha$ final de 4,92\%, (menor a 5\% para tener en cuenta el análisis no planificado previo a la finalización del estudio). El estudio finalmente se detuvo cuando la muestra estuvo compuesta por 235 pacientes.

El análisis por intención de tratar (ITT) incluyó todos los casos aleatorizados. El análisis por protocolo (PP) incluyó solo los casos en los que se logró el cierre completo. También se realizó un análisis post hoc de las variables relacionadas con cierre completo, cierre parcial y cierre fallido. Las características de la muestra se describieron mediante estadística descriptiva; para las variables categóricas se utilizaron frecuencias y porcentajes y para las cuantitativas se utilizaron medias y desviaciones estándar. Las comparaciones entre grupos se evaluaron mediante pruebas $\mathrm{T}$ de Student o ANOVA para variables continuas y con la prueba de chi-cuadrado o la prueba exacta de Fisher para variables categóricas. La incidencia de HD dentro de cada grupo de tratamiento se estimó utilizando proporciones de muestra con IC del 95\%. Se utilizaron modelos de regresión logística univariante para estimar las OR para el sangrado tardío en los grupos según tratamiento. A modo complementario se ajustaron modelos de regresión logística multivariante incluyendo como covariables adicionales al grupo de tratamiento aquellas que fueron significativas en los modelos univariados proporcionando los OR ajustados. La significación estadística se consideró como un valor de p menor de 0,049. 


\section{A3.3 RESULTADOS}

Entre mayo de 2016 y junio de 2018 se reclutaron 237 casos de LCRNP en el estudio. Se excluyeron dos casos por perforación diferida, uno en cada grupo. Hubo 116 pacientes en el grupo de control y 119 en el grupo de cierre con clips. En la Figura A3.1 se muestra un diagrama de flujo.

Figura A3. 1. Diagrama de flujo

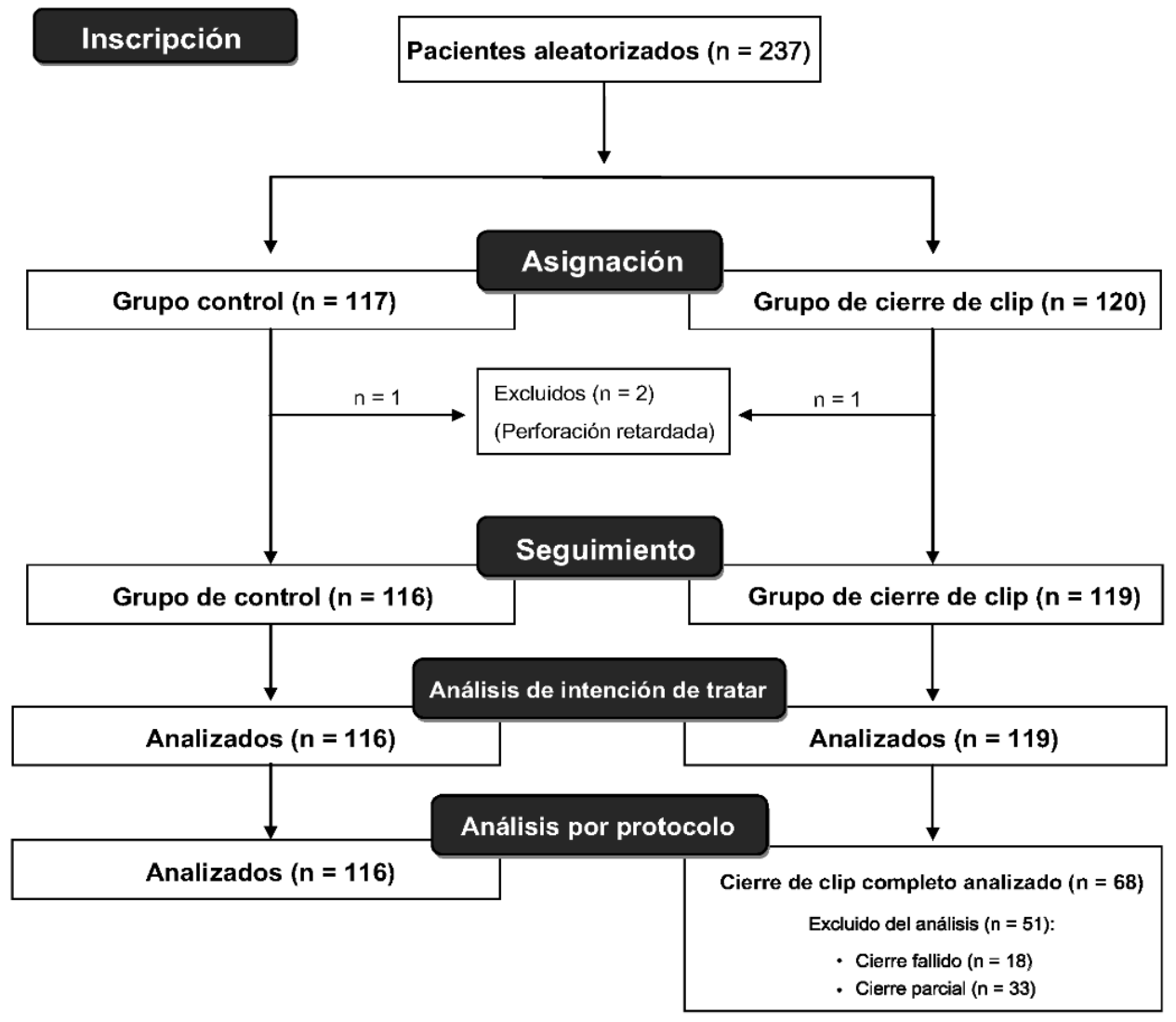

Al comparar las características basales entre los grupos de control y de tratamiento, hubo diferencias en el tratamiento antiagregante, que se utilizó con más frecuencia en el grupo de cierre con clips. No hubo otras diferencias estadísticamente significativas entre las características de los pacientes o las características de los LCRNP (Tabla A3.1). 
Tabla A3.1. Características basales de los pacientes asignados a cada grupo.

\begin{tabular}{|c|c|c|c|c|}
\hline Variable & Categoría & $\begin{array}{c}\text { Control } \\
116(49.4 \%)\end{array}$ & $\begin{array}{l}\text { Tratamiento } \\
119(50.6 \%)\end{array}$ & $\begin{array}{r}\text { Valor } \\
p\end{array}$ \\
\hline \multicolumn{5}{|c|}{ Características demográficas } \\
\hline Edad (años) & & $71.1(9.0)$ & 72.7 (8.7) & 0.169 \\
\hline \multirow[t]{2}{*}{ Grupo Edad (años) } & $<75$ & $73(62.9 \%)$ & $61(51.3 \%)$ & 0.071 \\
\hline & $\geq 75$ & $43(37.1 \%)$ & $58(48.7 \%)$ & \\
\hline \multirow[t]{2}{*}{ Sexo } & Masculino & 77 (66.4\%) & $81(68.1 \%)$ & 0.783 \\
\hline & Femenino & $39(33.6 \%)$ & 38 (31.9\%) & \\
\hline \multicolumn{5}{|c|}{ Características clínicas } \\
\hline \multirow[t]{2}{*}{ ASA en grupos } & $I+\|$ & $58(50.0 \%)$ & 47 (39.5\%) & 0.105 \\
\hline & III+ IV & $58(50.0 \%)$ & $72(60.5 \%)$ & \\
\hline \multirow[t]{2}{*}{ Hipertensión } & No & $44(37.9 \%)$ & $38(31.9 \%)$ & 0.335 \\
\hline & $\mathrm{Si}$ & $72(62.1 \%)$ & $81(68.1 \%)$ & \\
\hline \multirow[t]{2}{*}{ Antiagregantes } & No & $82(70.7 \%)$ & $69(58 \%)$ & $0.042 *$ \\
\hline & $\mathrm{Si}$ & $34(29.3 \%)$ & $50(42.0 \%)$ & \\
\hline \multirow[t]{2}{*}{ Anticoagulantes } & No & $85(73.3 \%)$ & $89(74.8 \%)$ & 0.791 \\
\hline & $\mathrm{Si}$ & $31(26.7 \%)$ & $30(25.2 \%)$ & \\
\hline Terapia puente con & No & $6(19.4 \%)$ & $7(23.3 \%)$ & 0.947 \\
\hline heparina & $\mathrm{Si}$ & $25(80.6 \%)$ & $23(76.7 \%)$ & \\
\hline \multicolumn{5}{|c|}{ Características de la lesión } \\
\hline \multirow[t]{2}{*}{ Morfología } & Sésil & $46(39.7 \%)$ & 47 (39.5\%) & 0.980 \\
\hline & Plano & $70(60.3 \%)$ & $72(60.5 \%)$ & \\
\hline Tamaño(mm) & & $37.3(12.9)$ & $36.1(14.3)$ & 0.235 \\
\hline \multirow[t]{2}{*}{ Tamaño >40 mm } & No & $54(46.6 \%)$ & $66(55.5 \%)$ & 0.172 \\
\hline & $\mathrm{Si}$ & $62(53.4 \%)$ & $53(44.5 \%)$ & \\
\hline Colon proximal & No & $12(10.3 \%)$ & $10(8.4 \%)$ & 0.609 \\
\hline (trasverso incluido) & $\mathrm{Si}$ & 104 (89.7\%) & 109 (91.6\%) & \\
\hline Colon proximal & No & $28(24.1 \%)$ & $29(24.4 \%)$ & 0.967 \\
\hline (trasverso excluido) & $\mathrm{Si}$ & 88 (75.9\%) & 90 (75.6\%) & \\
\hline \multirow[t]{6}{*}{ Histología } & Adenoma-DBG & 68 (58.6\%) & $72(60.5 \%)$ & 0.413 \\
\hline & Adenoma-DAG & $36(31.0 \%)$ & $35(29.4 \%)$ & \\
\hline & ADC-ISmS & $5(4.3 \%)$ & $4(3.4 \%)$ & \\
\hline & Serrado-DBG & $7(6.0 \%)$ & $4(3.4 \%)$ & \\
\hline & Serrado-DAG & $0(0.0 \%)$ & $3(2.5 \%)$ & \\
\hline & Serrado Ca-IS & $0(0.0 \%)$ & $1(0.8 \%)$ & \\
\hline Histología de & No & 71 (63.4\%) & $68(61.3 \%)$ & 0.743 \\
\hline alto riesgo & $\mathrm{Si}$ & $41(36.6 \%)$ & $43(38.7 \%)$ & \\
\hline
\end{tabular}

Descrito como la media y desviación estándar (DE). Displasia de bajo grado (DBG), Displasia de alto grado (DAG); ISmS: Invasión submucosa superficial; Ca: Carcinoma. 
En el grupo de clips, se logró el cierre completo en 68 pacientes (57\%) y el cierre parcial en 33 pacientes (28\%). El cierre fallido ocurrió en los 18 pacientes restantes (15\%). La mediana del número de clips necesarios para el cierre completo fue de 6 (rango, 3-13).

La coagulación con la punta del asa se utilizó en 44 (38\%) casos en el grupo de control y en $50(42 \%)$ casos en el grupo de clips $(p=0,61)$. La coagulación APC se utilizó en 9 (8\%) casos en el grupo de control y en 10 (8\%) casos en el grupo de clips $(p=0,94)$. Se utilizaron pinzas de coagulación en $2(2 \%)$ casos en el grupo de control y en $3(3 \%)$ casos en el grupo de clips $(p=1)$. En el grupo control 9 pacientes (8\%) requirieron clips de forma puntual por sangrado inmediato $y$ ninguno de los casos recibió un cierre completo.

Del total de 235 pacientes, hubo HD en 20 pacientes (8,5\%); 14 (12,1\%) HD en el grupo de control y 6 (5\%) en el grupo de clips (Tabla A3.2). Siete casos ocurrieron en las primeras 48 horas; 8 casos ocurrieron entre 3 y 7 días después de la RME y 5 casos ocurrieron en la segunda semana después de la resección., Ningún paciente requirió intervención quirúrgica o angiográfica para el control de la HD. Se efectuó una endoscopia adicional en 15 de 20 pacientes (75\%): 2 pacientes del grupo de clips fueron tratados con dos métodos hemostáticos, 8 pacientes del grupo de control también recibieron intervención endoscópica (7 pacientes tratados con clips y uno con dos métodos) y 5 pacientes no recibieron ningún tratamiento. Cuatro pacientes requirieron transfusiones. Los 20 casos de hemorragia tardía representaron un total de 61 días de hospitalización (mediana 3; rango, 1 a 7 días). 
En el análisis ITT el riesgo de HD fue del 12,1\% en el grupo de control y del 5\% en el grupo de clips (Tabla A3.2 y Figura A3.2), con una diferencia de riesgo absoluto (ARD) de $-7 \%$ (IC 95\%: $-14,7 \%, 0,3 \% ; p=0,053$ ) y un OR no ajustado de 0,39 (IC 95\%: 0,14; 1,04). El número necesario a tratar (NNT) fue 14.

Tabla A3.2. Resultados primarios y secundarios del análisis ITT y PP.

\begin{tabular}{|c|c|c|c|c|}
\hline & & Control & Tratamiento & Valor $p$ \\
\hline \multicolumn{5}{|c|}{ Hemorragia diferida } \\
\hline \multirow[t]{2}{*}{ ITT } & $\mathrm{n}(\%)$ & $14(12.1 \%)$ & $6(5.0 \%)$ & 0.053 \\
\hline & OR & Referencia & $0.39(0.14,1.04)$ & 0.061 \\
\hline \multirow[t]{2}{*}{ PP } & $\mathrm{n}(\%)$ & $14(12.1 \%)$ & $1(1.5 \%)$ & $0.011^{*}$ \\
\hline & OR & Referencia & $0.11(0.01,0.85)$ & $0.034^{*}$ \\
\hline \multicolumn{5}{|c|}{ Hemorragia intraprocedimiento } \\
\hline \multirow[t]{2}{*}{ ITT } & $\mathrm{n}(\%)$ & $18(15.5 \%)$ & $21(17.6 \%)$ & 0.661 \\
\hline & OR & Referencia & $1.17(0.59,2.32)$ & 0.661 \\
\hline \multirow[t]{2}{*}{ PP } & $\mathrm{n}(\%)$ & $18(15.5 \%)$ & $10(14.7 \%)$ & 0.882 \\
\hline & OR & Referencia & $0.94(0.41,2.17)$ & 0.882 \\
\hline \multicolumn{5}{|c|}{ Síndrome post-polipectomía } \\
\hline ITT & $\mathrm{n}(\%)$ & $0(0 \%)$ & $3(2.5 \%)$ & 0.247 \\
\hline PP & $\mathrm{n}(\%)$ & $0(0 \%)$ & $1(1.5 \%)$ & 0.370 \\
\hline \multicolumn{5}{|c|}{ Perforación } \\
\hline ITT & $\mathrm{n}(\%)$ & $1(0.9 \%)$ & $1(0.8 \%)$ & 0.999 \\
\hline PP & $\mathrm{n}(\%)$ & $1(0.9 \%)$ & $0(0 \%)$ & 0.999 \\
\hline \multicolumn{5}{|c|}{ Fiebre } \\
\hline ITT & n (\%) & 1 (0.9\%) & $1(0.8 \%)$ & 0.999 \\
\hline PP & n (\%) & 1 (0.9\%) & $0(0 \%)$ & 0.999 \\
\hline \multicolumn{5}{|c|}{ Dolor } \\
\hline ITT & n (\%) & $2(1.7 \%)$ & $6(5.0 \%)$ & 0.281 \\
\hline PP & $\mathrm{n}(\%)$ & $2(1.7 \%)$ & $2(2.9 \%)$ & 0.627 \\
\hline
\end{tabular}

ITT: análisis por intención de tratar (control vs tratado). PP: análisis por protocolo (control vs cierre completo). 
Fig. A3.2. Riesgo de HD en el análisis por intención de tratar (ITT) (control vs.

tratados) y en el análisis por protocolo (PP) (control vs. cierre completo).
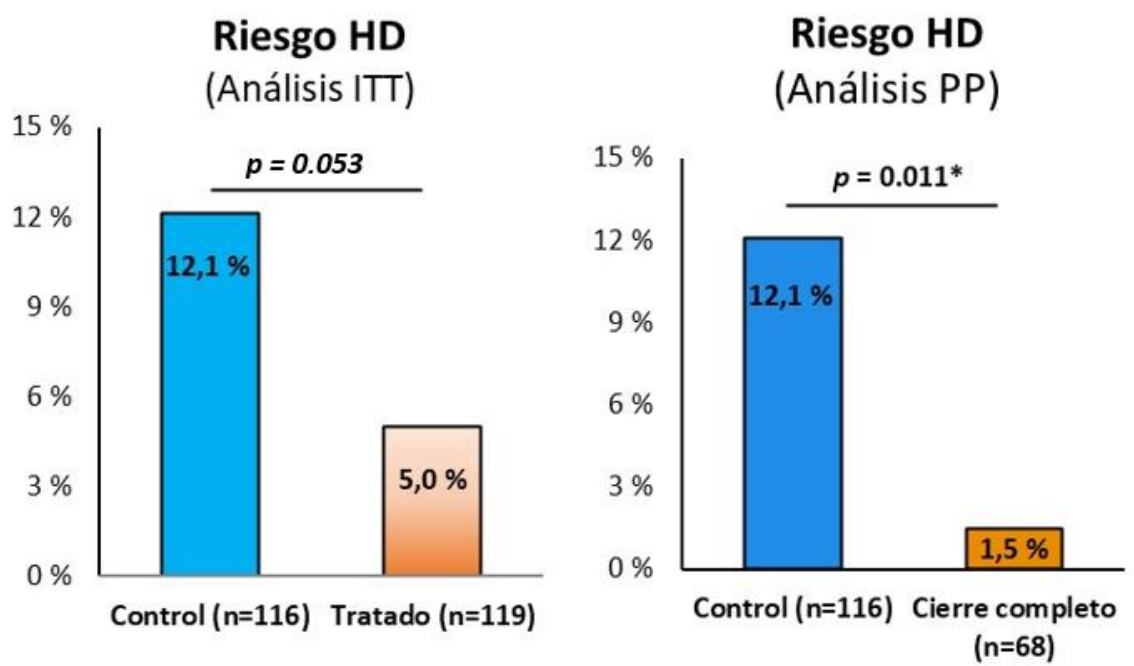

Para complementar el resultado principal del análisis ITT, se realizó un análisis multivariado ajustando por la variable de confusión "tratamiento antiagregante" obteniendo un OR ajustado de 0,30 (IC 95\%: 0,09-0,073; $p=$ 0,022). En el análisis del PP, el riesgo de DB fue del 12,1\% en el grupo control y del $1,5 \%$ en el grupo de cierre completo, con una ARD (absolute risk difference) de $-10,6 \%$ (IC 95\%: -17,9\%, -2,6\%; $p=0,011$ ) y una OR de 0,11 (IC 95\%: 0,01$0,061 ; p=0,015)$. Se realizó un análisis post hoc del resultado primario en los casos agrupados según varios factores de riesgo (Tabla A3.3).

El riesgo de HD entre los subgrupos del grupo de clips fue de 1,5\% en el subgrupo de cierre completo, $9,1 \%$ en el subgrupo de cierre parcial y $11 \%$ en el subgrupo de cierre fallido. Los pólipos que se cerraron completamente con éxito eran más pequeños, tenían mejor accesibilidad y el procedimiento de la RME fue más corto y sencillo subjetivamente. 
Tabla A3.3. Análisis de HD por subgrupos de factores de riesgo.

\begin{tabular}{|c|c|c|c|c|}
\hline & $\begin{array}{l}\text { Control } \\
\mathrm{N}=116\end{array}$ & $\begin{array}{l}\text { Tratamiento } \\
\qquad \mathrm{N}=119\end{array}$ & Valor $p$ & $\begin{array}{c}\text { ARD } \\
\text { (IC 95\%) }\end{array}$ \\
\hline \multicolumn{5}{|l|}{ Tamaño } \\
\hline $20-39 \mathrm{~mm}$ & $9 / 54$ (16.7\%) & $5 / 66(7.6 \%)$ & 0.123 & $-9.1(20.9,2.72)$ \\
\hline$\geq 40 \mathrm{~mm}$ & $5 / 62(8.1 \%)$ & $1 / 53$ (1.9\%) & 0.215 & $-6.2(-13.8,1.53)$ \\
\hline \multicolumn{5}{|l|}{ ASA } \\
\hline ASA I+II & $4 / 58(6.9 \%)$ & $1 / 47(2.1 \%)$ & 0.377 & $-4.8(-12.5,2.95)$ \\
\hline ASA III+IV & $10 / 58(17.2 \%)$ & $5 / 72$ (6.9\%) & 0.068 & $-10.3(-21.6,1.06)$ \\
\hline \multicolumn{5}{|c|}{ Localización proximal } \\
\hline No & $3 / 12$ (20.0\%) & $1 / 10(10.0 \%)$ & 0.594 & $-15(-45.7,15.7)$ \\
\hline Si & $11 / 104$ (10.6\%) & $5 / 109(4.6 \%)$ & 0.097 & $-6(-13.1,1.11)$ \\
\hline \multicolumn{5}{|c|}{ Antiagregantes } \\
\hline No & $6 / 82(7.3 \%)$ & $1 / 69$ (1.4\%) & 0.126 & $-5.87(-12.2,0.43)$ \\
\hline $\mathrm{Si}$ & $8 / 34(23.5 \%)$ & $5 / 50(10 \%)$ & 0.092 & $-13.5(-30.0,2.97)$ \\
\hline \multicolumn{5}{|l|}{ Grupo edad } \\
\hline 50-74 años & $6 / 73(8.2 \%)$ & 1/61 (1.6\%) & 0.126 & $-6.6(-13.6,0.48)$ \\
\hline$\geq 75$ años & $8 / 43(18.6 \%)$ & $5 / 58(8.6 \%)$ & 0.138 & $-10(-23.67,3.707)$ \\
\hline
\end{tabular}

La hemorragia intraprocedimiento ocurrió en 18 pacientes (16\%) y 21 (18\%) en los grupos control y clips respectivamente $(p=0,79)$ y se trató principalmente con coagulación con la punta del asa. Cuatro casos con sangrado inmediato también desarrollaron HD. No hubo casos de síndrome postpolipectomía en el grupo control pero sí 3 casos en el grupo de clips $(p=0,25)$. De estos 3 casos, se hizo un cierre completo en 1 y fue fallido en los otros 2 casos. Todos los pacientes evolucionaron favorablemente con analgesia y antibióticos de amplio espectro. Solo 2 pacientes presentaron fiebre post- 
resección (un paciente en cada grupo). Finalmente, 2 (2\%) pacientes en el grupo de control y 6 (5\%) pacientes en el grupo de clips presentaron dolor que requirió intervención médica $(p=0,28)$. No hubo ningún fallecimiento.

\section{A3.4 CONCLUSIONES}

-En este ensayo clínico el cierre completo de los defectos mucosos postresección de LCRNP con riesgo sustancial de sangrado diferido redujo dicho riesgo; se observó una clara tendencia en el análisis ITT y un efecto claramente significativo al ajustar por la variable de tratamiento antiagregante.

-Se observó un efecto protector evidente en aquellos casos en los que se logró el cierre completo del clip. Sin embargo este cierre completo solamente se consiguió en el 57\% de los casos.

- Nuestro estudio, a diferencia de todos los realizados previamente, ha incluido solo lesiones con riesgo sustancial de HD ( $\geq 8 \%$ ). Más del $90 \%$ de los casos eran pólipos proximales y el 51\% eran mayores de $40 \mathrm{~mm}$; además, el $36 \%$ de los pacientes estaban tomando antiagregantes plaquetarios. Combinamos esas variables utilizando la puntuación GSEED-RE, lo que resultó en un porcentaje de $\mathrm{HD}$ del $12,1 \%$ en el grupo de control, que fue más alta que la informada en otros estudios. 


\section{A3.5 PUBLICACIÓN DEL TRABAJO}

Los resultados del estudio se presentaron en el congreso “ESGE days 2019" en Praga y se publicaron en forma de abstract en la revista Endoscopy (Endoscopy 2019; 51: S32) ${ }^{116}$. Posteriormente se presentaron en el congreso nacional de la Sociedad Española de Patología Digestiva, obteniendo el premio a la mejor comunicación oral en endoscopia digestiva y se publicaron en la Revista Española de Enfermedades Digestivas en formato abstract (REED 2019). La publicación final del artículo 3 (ver apéndice) ha sido en la revista Gastroenterology (FI 2019: 17.373) ${ }^{117}$. 


\section{ARTíCULO 4. Cierre con clips para la prevención} de hemorragia diferida tras RME colorrectal. Metaanálisis de ensayos clínicos randomizados.

\section{A4.1 INTRODUCCIÓN Y OBJETIVOS}

Las evidencias que apoyan el cierre profiláctico con hemoclips para prevenir la HD post-resección han sido controvertidas hasta el año 2019. Por ejemplo, la ESGE sugería que esta medida podía tener un papel individualizando la decisión en función de los factores de riesgo del paciente. Sin embargo, esta recomendación ha sido calificada como débil por estar basada en evidencias de calidad subóptima.

En 2019, se publican en Gastroenterology 3 ensayos clínicos al respecto (Feagins et $\mathrm{al}^{118}$, Pohl et $\mathrm{al}^{119}$ y Albéniz et $\mathrm{al}^{120}$ (trabajo previo denominado ARTICULO 3)).

El trabajo de Feagins et $\mathrm{al}^{118}$, un estudio de equivalencia con dos ramas, no encontró diferencias significativas en el riesgo de HD del grupo control, si bien sólo el 39\% de las lesiones incluidas era mayor de dos centímetros y mezclaba pólipos pediculados con no pediculados.

El ensayo clínico de Pohl et $a^{119}$, randomizó 928 pacientes con una metodología adecuada. Se produjo HD en el 3,5\% de los pacientes en el grupo de 
clips y en el 7,1\% del grupo control. Con respecto a los pólipos localizados en el colon proximal, el riesgo de sangrado en el grupo de clips fue del 3,3\% frente al 9.6\% en los controles. Ambas diferencias fueron estadísticamente significativas (ARD, 6.3\%; 95\% Cl, 2.5\%-10.1\%).

Debido a las nuevas evidencias aportadas estos últimos años, se realizó una revisión sistemática y un metaanálisis de todos los ensayos clínicos disponibles con el objetivo primario de aclarar el papel profiláctico de los hemoclips en la prevención de la HD tras la resección endoscópica de lesiones colorrectales.

El objetivo secundario fue determinar el efecto de las variables de estudio sobre el riesgo de HD con y sin cierre con hemoclips. Las variables de estudio que se tuvieron en cuenta fueron: edad y sexo de los pacientes, porcentaje de pacientes en tratamiento antiagregante/anticoagulante, tamaño, morfología pediculada o sésil, la localización y el porcentaje de lesiones serradas o adenomatosas.

\section{A4.2 PACIENTES Y MÉTODOS}

Los métodos y criterios de inclusión se basaron en las recomendaciones para revisiones sistemáticas y metaanálisis recogidas en PRISMA. El protocolo de revisión sistemática se registró en el Registro internacional prospectivo de revisiones sistemáticas (PROSPERO, www.crd.york.ac.uk/prospero/) en octubre de 2019. 
Se examinaron todos los estudios clínicos que cumplieron los siguientes criterios de inclusión:(I) Población: todos los adultos sometidos a resección endoscópica de lesiones colorrectales; (II) Intervención: cierre profiláctico de defectos mucosos post-resección; (III) Comparación: no cierre del defecto mucoso; (IV) Resultado: riesgo de HD; (V) Diseño del estudio: solo se consideraron los ensayos controlados aleatorios.

Los criterios de exclusión fueron los siguientes: (I) Información esencial no disponible; (II) Estudios no publicados como artículo de texto completo; (III) Estudios no publicados en idioma inglés; (IV) Estudios que consideren como comparador cualquier otra estrategia para la profilaxis de hemorragias postresección (argón, inyección de esclerosantes, uso de agentes hemostáticos tópicos, etc.); (V) estudios que incluyan menos de 10 pacientes por brazo.

Como resumen de la metodología, se realizó una búsqueda bibliográfica en las bases de datos de Medline/PubMed, EMBASE y Scopus para identificar ensayos randomizados originales. Se utilizó el metanálisis de efectos aleatorios para generar el riesgo relativo (RR) agrupado con IC del 95\%. Se utilizó un análisis de metarregresión de efectos aleatorios multinivel para combinar los riesgos de HD y estimar las asociaciones entre éstos y las características de los pólipos.

\section{A4.3 RESULTADOS}

La búsqueda bibliográfica inicial fue de 1112 artículos, de los que se incluyeron un total de 9 ensayos en el análisis final (Tabla A4.1). 
El número total de participantes incluidos en el análisis ITT fue de 4557 . Se analizaron un total de 7.197 lesiones colorrectales (3.544 grupo clips y 3.653 grupo control). Hubo 3 ensayos que utilizaron un tamaño de $20 \mathrm{~mm}$ como punto de corte para la inclusión y 3 estudios que incluyeron cualquier tamaño de pólipo (porcentaje medio de lesiones $\geq 20 \mathrm{~mm}, 19,4 \%$ ), mientras que los 3 estudios restantes solo incluyeron lesiones de tamaño $<20 \mathrm{~mm}$.

Tabla A4.1 - Características de los estudios.

\begin{tabular}{cccccc}
\hline Estudio & País & $\begin{array}{c}\text { Pacientes } \\
\text { (clips/no } \\
\text { clips) }\end{array}$ & $\begin{array}{c}\text { N pólipos } \\
\text { (clips/no } \\
\text { clips) }\end{array}$ & $\begin{array}{c}\text { Lesiones de } \\
\text { gran tamaño } \\
\text { (clips/no clips) }\end{array}$ & $\begin{array}{c}\text { Lesiones } \\
\text { proximales } \\
\text { (clips/no clips) }\end{array}$ \\
\hline $\begin{array}{c}\text { Albeniz } \\
(2019)\end{array}$ & España & 235 & 235 & 235 & 213 \\
\hline Dokoshi & Japón & 157 & 288 & 14 & $(109 / 104)$ \\
(2015) & & $(89 / 68)$ & $(154 / 134)$ & $(8 / 6)$ & 74 \\
\hline Feagins & USA & 1050 & 1386 & 222 & $(40 / 34)$ \\
\hline (2019) & & $(530 / 520)$ & $(680 / 706)$ & $(101 / 121)$ & $(261 / 275)$ \\
\hline Matsumoto & USA & 1499 & 3364 & 0 & 1668 \\
(2016) & & $(752 / 747)$ & $(1636 / 1728)$ & $(0 / 0)$ & $(823 / 845)$ \\
\hline Mori & Japón & NA & 148 & 0 & 42 \\
(2015) & & & $(73 / 75)$ & $(0 / 0)$ & $(21 / 21)$ \\
\hline Osada & Japón & 26 & 26 & 26 & 18 \\
(2016) & & $(13 / 13)$ & $(13 / 13)$ & $(13 / 13)$ & $(8 / 10)$ \\
\hline Pohl & USA & 919 & 989 & 989 & 658 \\
\hline (2019) & & $(455 / 464)$ & $(490 / 499)$ & $(490 / 499)$ & $(327 / 331)$ \\
\hline Shioji & Japón & 323 & 413 & 0 & 187 \\
(2003) & & $(156 / 167)$ & $(205 / 208)$ & $(0 / 0)$ & $(97 / 90)$ \\
\hline Zhang & China & 348 & 348 & 130 & 101 \\
(2015) & & $(174 / 174)$ & $(174 / 174)$ & $(63 / 67)$ & $(50 / 51)$ \\
\hline
\end{tabular}

Según los datos informados por los 9 ensayos las estimaciones del riesgo de HD entre los grupos de control y de clips fue del $3,3 \%$ (IC del $95 \%, 1,9-5,7)$ y 
2,2\% (IC del 95\%, 1,2-3,9). No hubo diferencias significativas entre los grupos de tratamiento y control en el riesgo de HD cuando se aplicó un modelo de efectos aleatorios ( $R R=0,69$, IC del 95\%: 0,45-1,08, $p=0,072$, Figura A4.1).

Figura A4.1: Diagrama de bosque que compara el brazo de cierre con clips vs grupo control.

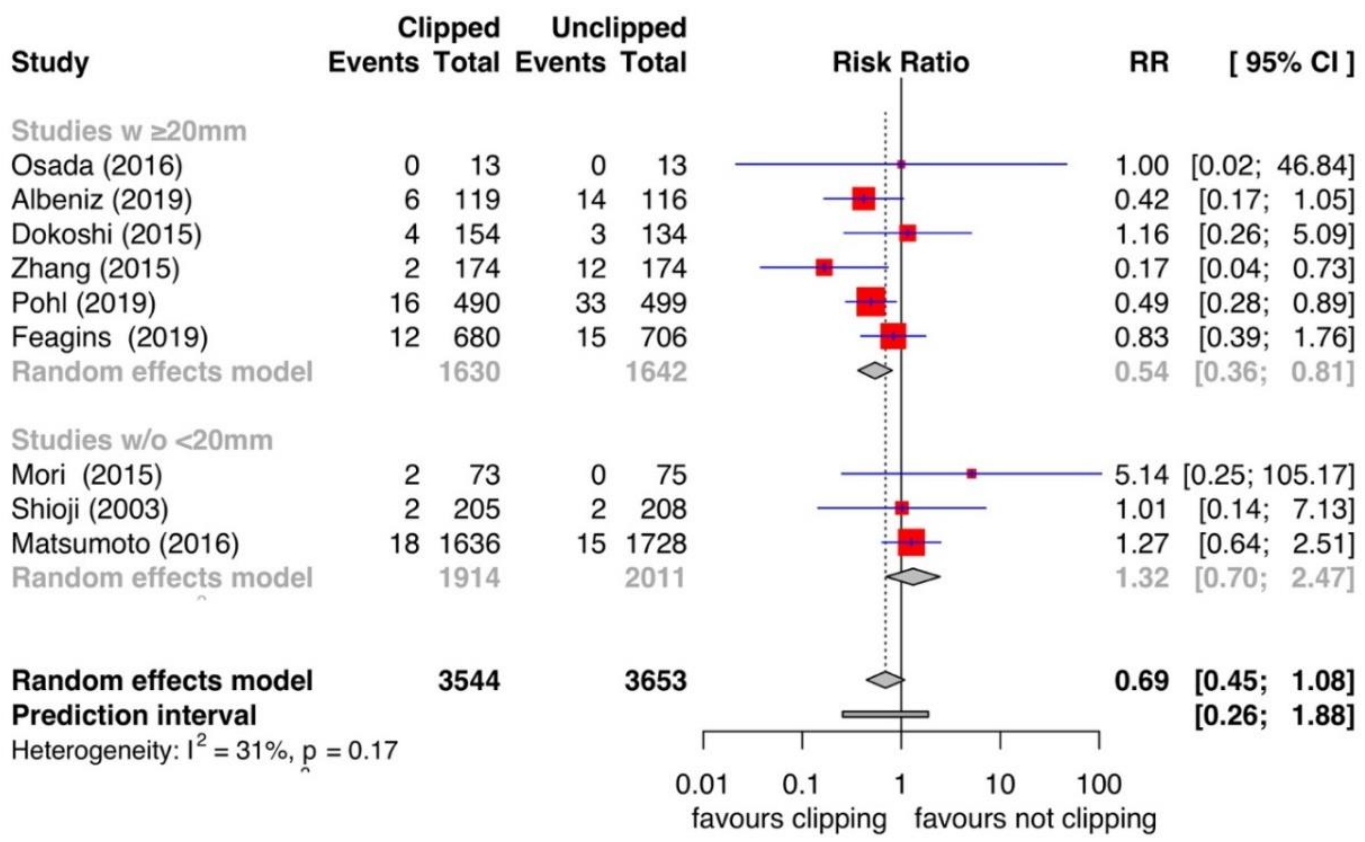

Los resultados del análisis de sensibilidad "leave-one-out" se muestran en el material suplementario. Solo un estudio que evaluó pacientes con lesiones $<20$ mm tuvo una influencia sustancial (Matsumoto et al, 2016) ${ }^{101}$; después de eliminar este ensayo, la heterogeneidad se redujo significativamente $\left(I^{2}=8,2 \%\right)$.

Para investigar el impacto de las características del estudio en las estimaciones de RR, realizamos un análisis de meta-regresión (Tabla A4.2). 
Tabla A4.2 Metanálisis del riesgo general de HD - Resultados de la metaregresión.

\begin{tabular}{|c|c|c|c|c|c|c|}
\hline \multirow[b]{2}{*}{ Variable } & \multicolumn{3}{|c|}{$\begin{array}{c}\text { Meta-regresión } \\
\text { univariante }\end{array}$} & \multicolumn{3}{|c|}{$\begin{array}{c}\text { Meta-regresión } \\
\text { multivariante }\end{array}$} \\
\hline & $\mathbf{R R}$ & IC 95\% & $p$ & $\mathbf{R R}$ & IC 95\% & $p$ \\
\hline $\mathbf{N}$ & 1.03 & $1.00-1.06$ & 0.051 & 1.01 & $0.98-1.05$ & 0.438 \\
\hline \multicolumn{7}{|l|}{ Edad media } \\
\hline$<66$ años & 1 & & & & & \\
\hline$>65$ años & 0.54 & $0.23-1.26$ & 0.155 & & & \\
\hline \multicolumn{7}{|l|}{ País } \\
\hline USA/Europa & 1 & & & & & \\
\hline Asia & 0.99 & $0.33-3.00$ & 0.991 & & & \\
\hline Hombres (\%) & 0.99 & $0.40-0.33$ & 0.957 & & & \\
\hline $\begin{array}{l}\text { Lesiones de gran } \\
\text { tamaño (\%) }\end{array}$ & 0.92 & $0.85-0.98$ & 0.017 & 0.92 & $0.86-0.99$ & 0.042 \\
\hline $\begin{array}{l}\text { Lesiones } \\
\text { proximales (\%) }\end{array}$ & 0.88 & $0.74-1.05$ & 0.152 & & & \\
\hline $\begin{array}{l}\text { Lesiones no } \\
\text { polipoides (\%) }\end{array}$ & 1.27 & $0.21-7.54$ & 0.792 & & & \\
\hline $\begin{array}{l}\text { Lesiones no } \\
\text { pediculadas (\%) }\end{array}$ & 0.14 & $0.01-4.21$ & 0.256 & & & \\
\hline Adenomas (\%) & 1.10 & $0.78-1.52$ & 0.680 & & & \\
\hline Serrados (\%) & 0.11 & $0.00-5.72$ & 0.276 & & & \\
\hline $\begin{array}{l}\text { Pacientes } \\
\text { antiagregados (\%) }\end{array}$ & 0.02 & $0.00-3.78$ & 0.144 & & & \\
\hline
\end{tabular}

El análisis indicó que el porcentaje de lesiones grandes ( $\geq 20 \mathrm{~mm})$ fue la única variable estadísticamente significativa asociada con el riesgo de HD. Un análisis de subgrupos por tamaño de pólipo (pólipos grandes versus pólipos pequeños) confirmó el resultado de la meta-regresión (Figura A4.2). 
Figura A4.2. Análisis de subgrupos del riesgo relativo de desarrollar HD en el grupo de tratamiento y control para pólipos grandes versus pequeños.

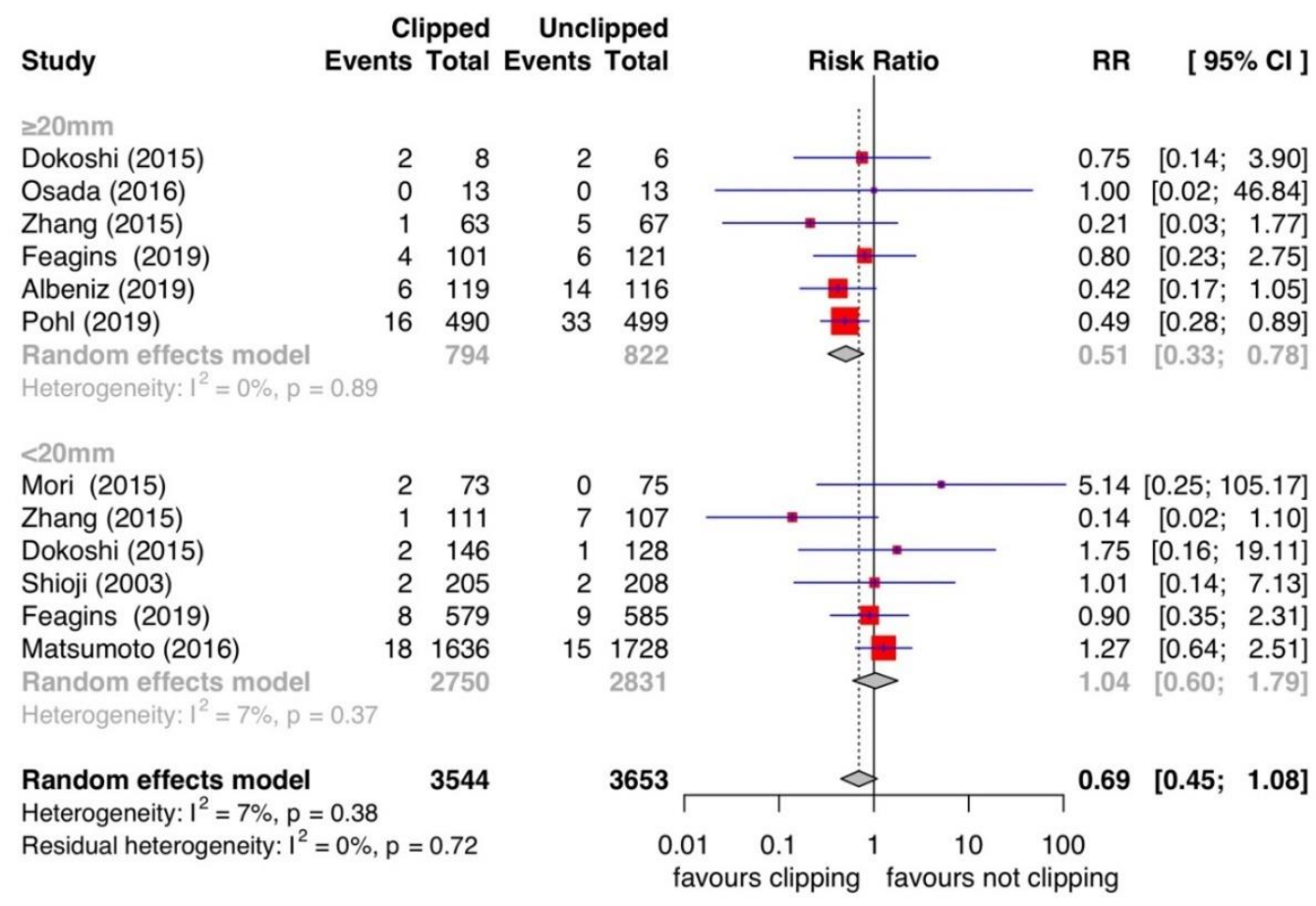

Entre las lesiones localizadas proximalmente hubo una asociación significativa entre el cierre completo de escaras y el riesgo de $H D(R R=0,53$; IC 95\%: 0,35-0,81). Sin embargo, entre las lesiones ubicadas distalmente no hubo diferencias significativas en cuanto al riesgo de hemorragia $(R R=1,01$; IC del 95\%: 0,43-2,37). Según el análisis de meta-regresión de efectos aleatorios multinivel, los riesgos estimados de HD en el colon distal y proximal fueron del 2,7\% (IC del 95\%: 1,3-5,4\%; IP: 0,5-12,3\%) y 6,2\% (IC del 95\%: 3,4-11,1\%; IP: 1,3$24,3 \%$ ), respectivamente, en el grupo de control versus 3,2\% (IC del 95\%: $1,6-$ 6,5\%; IP: 0,7-14,6\%) y 3,0\% (IC del 95\%: 1,5-5,7 \%; PI: 0,6-13,2\%), respectivamente, en el grupo de clips. Después de ajustar por tamaño, el 
beneficio del cierre con clips sólo se observó en las lesiones proximales de gran tamaño (Figura A4.3).

Figura A4.3. Análisis de subgrupos que compara el riesgo de HD del grupo control y el de tratamiento para lesiones ubicadas distal y proximalmente.

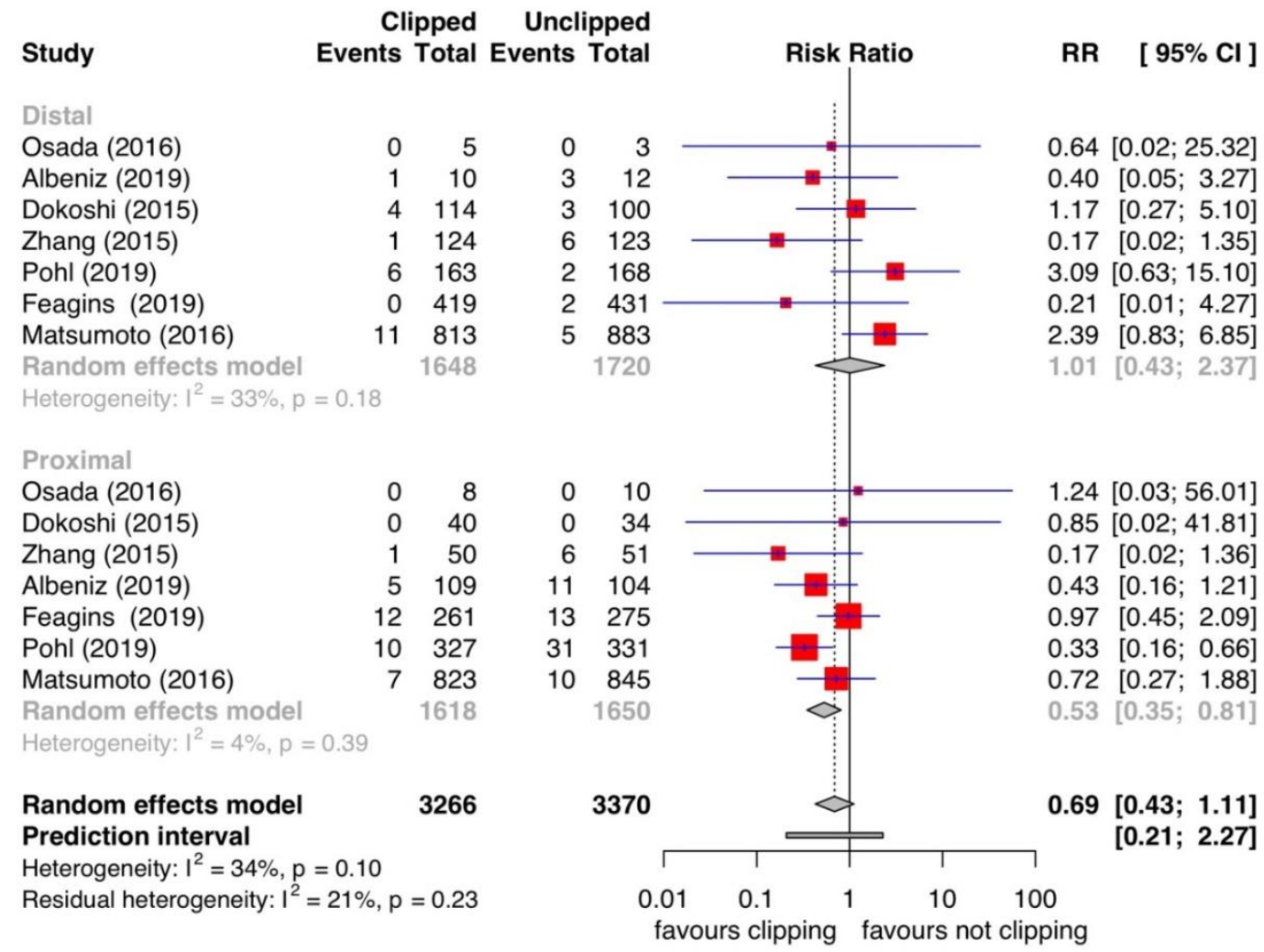




\section{A4.4 CONCLUSIONES}

-Según los resultados del metaanálisis, la práctica habitual del cierre completo de los defectos mucosos post-resección no reduce el riesgo de HD. Sin embargo, el cierre fue eficaz en reducir el riesgo de HD en casi un 50\% para las lesiones de gran tamaño (aquellas mayores o iguales de $2 \mathrm{~cm}$ ).

-El factor del tamaño es el único que muestra diferencias significativas en el análisis de meta-regresión.

-En cuanto al factor de la localización, la reducción del riesgo de HD que se consigue con el cierre profiláctico con clips solo se objetiva en las lesiones proximales de gran tamaño y no en las distales de gran tamaño.

- Este metaanálisis destaca por: el gran número de pólipos incluidos; por incluir un gran número de lesiones de gran tamaño gracias a las nuevas evidencias disponibles y por permitir un análisis leave-one out, ya que los trabajos previos no tenían la suficiente potencia estadística para hacerlo.

\section{A4.5 PUBLICACIÓN DEL TRABAJO}

Este trabajo fue aceptado como comunicación oral en la DDW, Chicago 2020 y publicado en forma de abstract en Gastrointestinal Endoscopy (GIE 2020, 91 6S: AB481). La publicación completa del estudio (ver Articulo 4 en el apéndice), en la que ocupo el puesto de "Co-first autor", se ha realizado en la revista Gastroenterology ( FI 2020: 17.373) ${ }^{121}$. 
upna 


\section{ARTíCULO 5. La respuesta a "cuándo cerrar con clips" después de la RME colorrectal, basada en un análisis de coste-efectividad.}

\section{A5.1 INTRODUCCIÓN Y OBJETIVOS}

Dos recientes ensayos controlados aleatorizados y multicéntricos mostraron un efecto beneficioso del cierre con clips en las LCRNP ubicadas en el colon proximal o con un riesgo sustancial de $\mathrm{HD}^{119,120}$ De la misma manera, el metaanálisis publicado recientemente concluyó que el cierre con clips del defecto mucoso post-resección previene la hemorragia en pacientes con LCRNP de gran tamaño proximales ${ }^{121}$. A pesar de que la evidencia respalda esta medida profiláctica, existen pocos trabajos y con resultados contradictorios en cuanto a su $\mathrm{CE}^{122-124}$. El trabajo de Parikh et al. concluye que la técnica es rentable sólo en pacientes que recibieron tratamiento anticoagulante ${ }^{122}$. Bahin et al. realizan un modelo económico en el que el cierre con clips no es rentable, se aplique de forma universal o selectivamente en lesiones proximales ${ }^{123}$. El trabajo más reciente es el de Shah et al., quienes concluyen que el cierre con clips supone un ahorro de costes en la población de Medicare (USA), aunque en el estudio se contabilizan los costes de las HD y no del cierre con clips $^{124}$.

El objetivo de nuestro trabajo fue evaluar el CE del cierre profiláctico con clips de los defectos mucosos después de la RME de LCRNP, tanto de forma global o universal en todos los pólipos, como de forma específica en el subgrupo 
de pacientes con alto riesgo estimado de HD y en dos escenarios económicos diferentes (Europa Occidental y Norte América).

\section{A5.2 PACIENTES Y MÉTODOS}

Se realizó un análisis de CE en base a tres fuentes: un estudio observacional prospectivo y multicéntrico que incluyó todas las RME de LCRNP registradas consecutivamente en la base de datos multicéntrica prospectiva del GSEED-RE (29 hospitales españoles) desde mayo de 2013 hasta julio de $2017(\mathrm{n}=2263)^{125}$, el metaanálisis previamente comentado ${ }^{121}$ y el ensayo clínico multicéntrico que mostró el beneficio del cierre preventivo con clips en pólipos con riesgo sustancial de $H D^{126}$. Los pacientes se clasificaron según el score GSEED-RE2 en tres grupos: grupo de riesgo bajo, 0-3 puntos; grupo de riesgo medio, 4-6 puntos; y grupo de alto riesgo, 7-9 puntos ${ }^{115}$. Todas las evaluaciones y supuestos asociados con el análisis de CE se derivaron para la cohorte total (TC) y para el subgrupo de HD de alto riesgo (HR) de GSEED-RE2. La medida de evaluación profiláctica fue el cierre de los defectos mucosos post-RME de LCRNP con clips y la variable resultado primaria fue el riesgo de HD.

Para evaluar el CE del cierre preventivo con clips realizado de forma universal a todas las lesiones (TC) o selectivo (HR) frente a la ausencia de profilaxis en dos entornos económicos diferentes (Europa Occidental y América del Norte) asumimos el árbol de decisiones que se muestra en la Figura A5.1. 
Figura A5.1. Árbol de decisiones

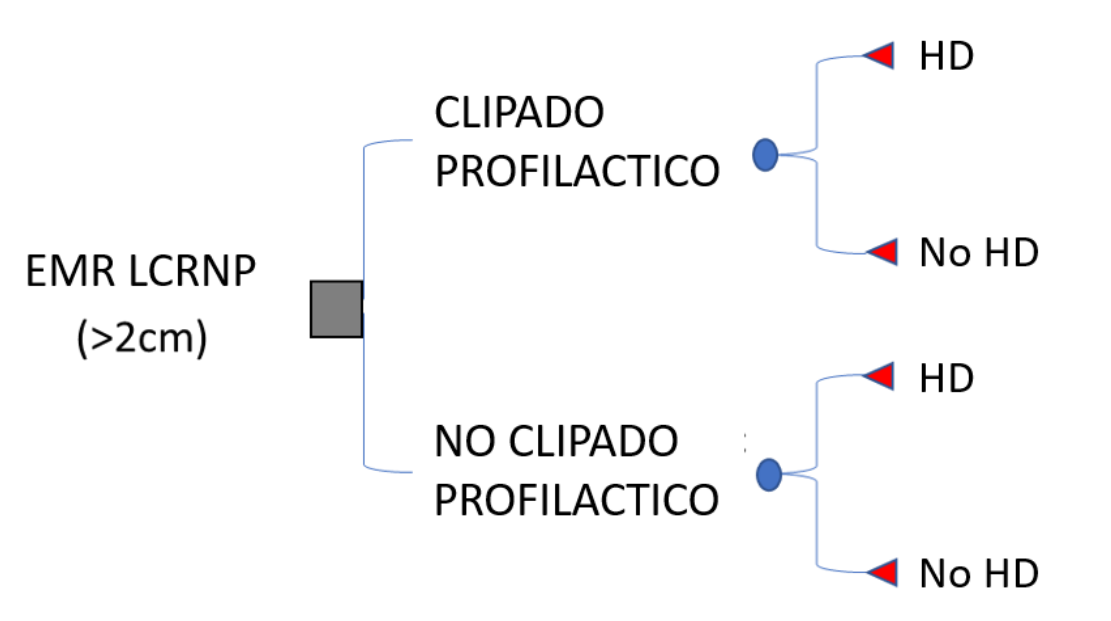

El coste del cierre del clip se calculó de la siguiente manera: [tamaño del pólipo ( $\mathrm{mm}$ ) / intervalo entre clips $(\mathrm{mm})]$ x precio del clip. Los precios de los clips se obtuvieron del mercado. La distancia intervalo entre clips $(6 \mathrm{~mm})$ y la reducción del riesgo relativo (RRR) de $\mathrm{HD}$ en pacientes con un riesgo sustancial de sangrado del $59 \%$ se obtuvieron de nuestro ensayo clínico ${ }^{120}$. La RRR de HD en la cohorte total (51\%) se obtuvo del metaanálisis previo ${ }^{121}$. En nuestro medio (Europa Occidental), el coste total de un episodio de HD se calculó a nivel de paciente sumando los costes de la atención médica urgente, la colonoscopia con sedación, el tratamiento de hemostasia endoscópica (es decir, pinza, inyección de adrenalina, pinzas de coagulación, coagulación con argón), embolización radiológica, transfusión, hospitalización y cirugía. El precio de todos los procedimientos relevantes se obtuvo del Sistema Nacional de Salud español ${ }^{127}$. Para el contexto norteamericano, se calculó un coste de HD promedio de $\$ 7500$ a partir de los estudios de Parikh et al. y Shah et al ${ }^{122,124}$. 
Solo se hicieron algunas suposiciones basadas en la bibliografía. La utilidad basal en nuestra cohorte (0,8333 años de vida ajustados por calidad, AVAC o QALYS) se obtuvo a partir de una gran encuesta de salud a nivel nacional ${ }^{128}$. La reducción en QALYs por un episodio de HD (0,0208 QALYs) se obtuvo del estudio de Doble et al ${ }^{129}$. El ratio del incremento coste-efectividad (ICER) que se utilizó para considerar una actuación como CE o no en nuestro medio se calculó como dos veces la Renta Nacional Bruta per cápita española en 2018 (54.000 € / QALY) como sugiere el proyecto CHOosing Interventions that are Cost-Effective (CHOICE) de la Organización Mundial de la Salud ${ }^{130}$. Se asumió un umbral de ICER de 100.000\$/QALY para considerar una actuación como CE o no en el entorno norteamericano. Finalmente, se incluyó una ICER de O€/QALY como umbral de ahorro de costes (CS).

El ICER para la colocación de los clips se determinó para las estrategias de cierre universal (TC) y selectivo (HR).

Los fondos necesarios para gastar (FNS) para evitar un episodio de HD se calcularon de la siguiente manera: FNS $=$ NNT * costo incremental/pólipo, donde NNT representa el número de lesiones que necesitan ser tratadas para evitar un episodio de HD.

Se realizaron análisis de sensibilidad unidireccionales y bidireccionales y análisis de umbral para evaluar la CE del cierre preventivo universal o selectivo en los contextos de Europa occidental y América del Norte. Para el análisis de sensibilidad unidireccional el ICER se representó frente al riesgo de HD. Para el análisis de sensibilidad bidireccional el riesgo de HD se representó gráficamente 
versus el intervalo del clip, el coste de un clip, el coste de un cierre completo con clips o el coste de un episodio de HD (fijando el umbral de CE en 54,000€/QALY o 100,000\$/QALY, según el contexto). Para el análisis de umbrales se consideraron un total de 8 escenarios utilizando dos tipos de cobertura (cierre universal o selectivo), dos contextos económicos (Europa occidental y Norteamérica) y dos umbrales de ICER diferentes (CE o CS).

\section{A5.3 RESULTADOS}

En total, se realizaron 2263 RME de LCRNP de gran tamaño de forma consecutiva en 2130 pacientes. El riesgo de HD en los pacientes de la TC fue del $4.5 \%$ y del $13,7 \%$ en el HR.

El análisis de CE se realizó utilizando los parámetros descritos en la Tabla A5.1 y los resultados se detallan en la Tabla A5.2. 
Tabla A5.1. Parámetros asumidos para el estudio CE.

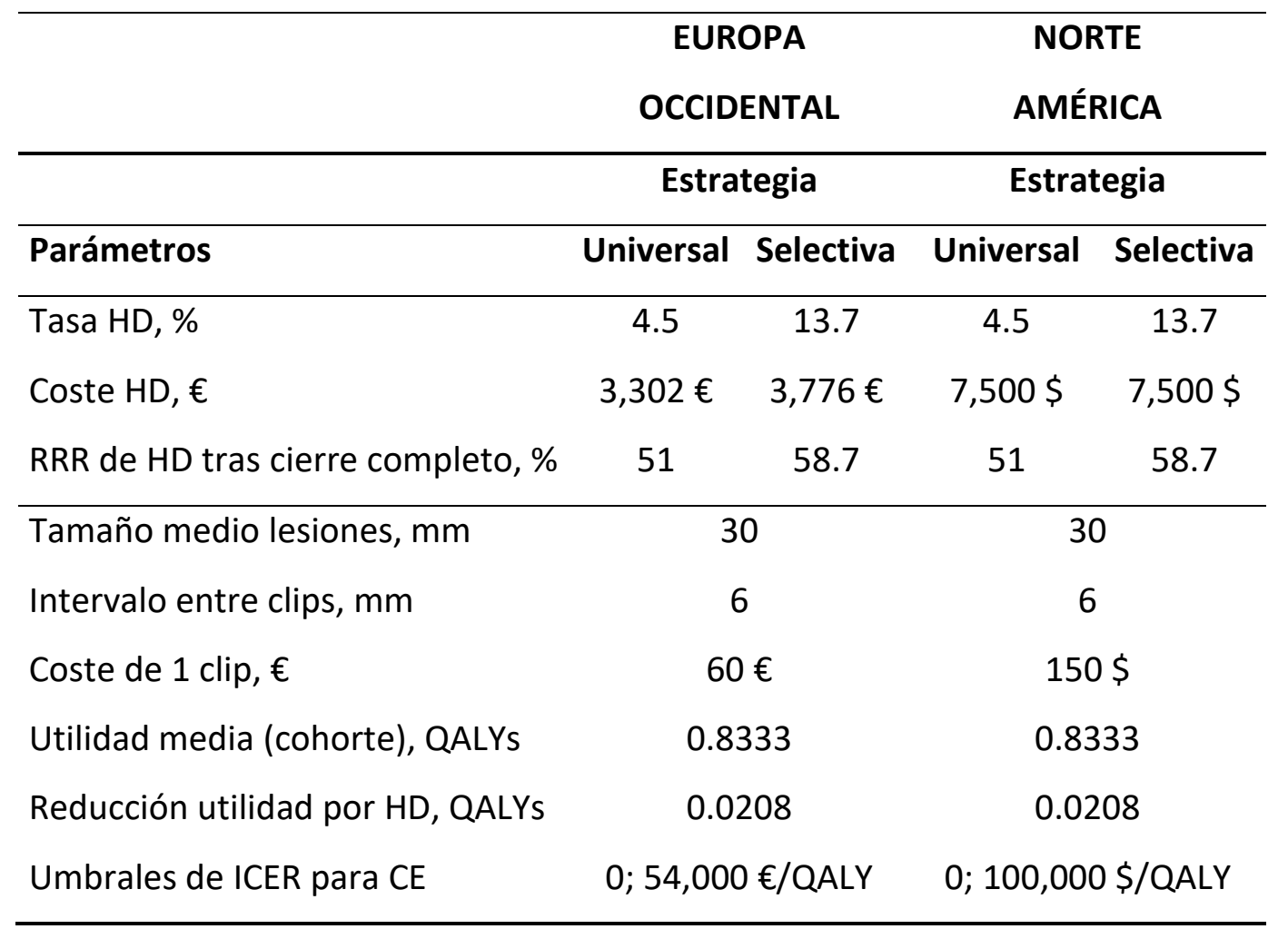

HD: hemorragia diferida; RRR: reducción del riesgo relativo; $Q A L Y$ : año de vida ajustado por calidad; ICER: Ratio del incremento coste-efectividad.

En nuestro medio, el cierre profiláctico universal supuso un coste incremental por pólipo de $224 €$, mientras que el cierre selectivo en la cohorte HR ahorró dinero (4 € por pólipo). El FNS para evitar un episodio de HD fue de $9865 €$ en el TC y -44 $€$ en el HR. Sin embargo, en la simulación de América del Norte, el costo incremental por pólipo fue de 578 \$ para el cierre universal y 147 $\$$ para el cierre selectivo. Los ICER resultantes para la estrategia de cierre universal en Europa o América del Norte, $469.706 €$ o $1.210 .564 \$$, respectivamente, no fueron CE. Por el contrario, el cierre selectivo en el HR supuso un ahorro de costes, con un ICER negativo de $-2194 €$ en el contexto de 
Europa occidental y fue CE con un ICER de 87.796 \$ en Norteamérica (Tabla A5.2).

Tabla A5.2. Análisis de costes y coste-efectividad del cierre profiláctico con clips en una estrategia universal y selectiva.

\begin{tabular}{|l|c|c|c|c|}
\hline & \multicolumn{2}{|c|}{ EUROPA OCCIDENTAL } & \multicolumn{2}{|c|}{ NORTE AMÉRICA } \\
\hline Estrategia: & Universal & Selectiva & Universal & Selectiva \\
\hline Riesgo de HD: & $4.5 \%$ & $13.7 \%$ & $4.5 \%$ & $13.7 \%$ \\
\hline Coste de HD: & $3,302 €$ & $3,776 €$ & $7,500 \$$ & $7,500 \$$ \\
\hline RRR: & $51.0 \%$ & $58.7 \%$ & $51.0 \%$ & $58.7 \%$ \\
\hline Coste de un clip: & $60 €$ & $60 €$ & $150 \$$ & $150 \$$ \\
\hline Medidas de costes & & & & \\
Coste de un cierre completo & $300 €$ & $300 €$ & $750 \$$ & $750 \$$ \\
Coste HD /pólipo si cierre & $73 €$ & $214 €$ & $165 \$$ & $424 \$$ \\
Coste HD /pólipo si no cierre & $149 €$ & $517 €$ & $338 \$$ & $1,028 \$$ \\
Coste total (HD+clips)/ pólipo & $373 €$ & $514 €$ & $915 \$$ & $1,174 \$$ \\
si cierre & & & & \\
Coste total (HD+clips)/ pólipo & $149 €$ & $517 €$ & $338 \$$ & $1,028 \$$ \\
si no cierre & $224 €$ & $-4 €$ & $578 \$$ & $147 \$$ \\
Coste incremental & & & & \\
Medidas de efectividad & $2.3 \%$ & $8.0 \%$ & $2.3 \%$ & $8.0 \%$ \\
$\quad$ RAR de HD & 44 & 12 & 44 & 12 \\
$\quad$ NNT & $9,770 €$ & $-46 €$ & $25,180 \$$ & $1,826 \$$ \\
$\quad$ FNS para prevenir una HD & & & & \\
$\quad$ Utilidad si cierre, QALYs & 0.832841 & 0.832123 & 0.832841 & 0.832123 \\
$\quad$ Utilidad si no cierre, QALYs & 0.832364 & 0.830450 & 0.832364 & 0.830450 \\
Efecto incremental, QALYs & 0.000477 & 0.001673 & 0.000477 & 0.001673 \\
ICER, $€$ & $469,707 €$ & $-2,194 €$ & $1,210,564 \$$ & $87,796 \$$ \\
aCoste efectivo & No & Yes & No & Yes \\
bAhorro de costes & Yes & No & Yes \\
\hline
\end{tabular}

RRR: reducción del riesgo relativo; RAR: Reducción absoluta del riesgo. NNT: Número necesario a tratar. FNS: Fondos necesarios para gastar para evitar un episodio de HD. QALY: año de vida ajustado por calidad. ICER: ratio del incremento CE. a, El umbral de $C E$ se estableció en 54000€/QALY (Europa occidental) o 100.000\$/QALY (Norteamérica). b, El umbral de CS era $0 \$$ /QALY. 
Los resultados del análisis de sensibilidad unidireccional se muestran en la Figura A5.2 y demuestran que el cierre universal es rentable cuando el riesgo de HD es superior al 13,3\% (Europa Occidental) o al 15,3\% (América del Norte). Sin embargo, para ambos entornos económicos el cierre selectivo es rentable cuando el riesgo de HD es superior al $10,5 \%$ o al $13,3 \%$, respectivamente.

Figura A5.2. Riesgo de HD vs ICER para las estrategias de cierre universal y selectivo en los contextos económicos de Europa (A) y Norteamérica (B).

A) Contexto Europeo

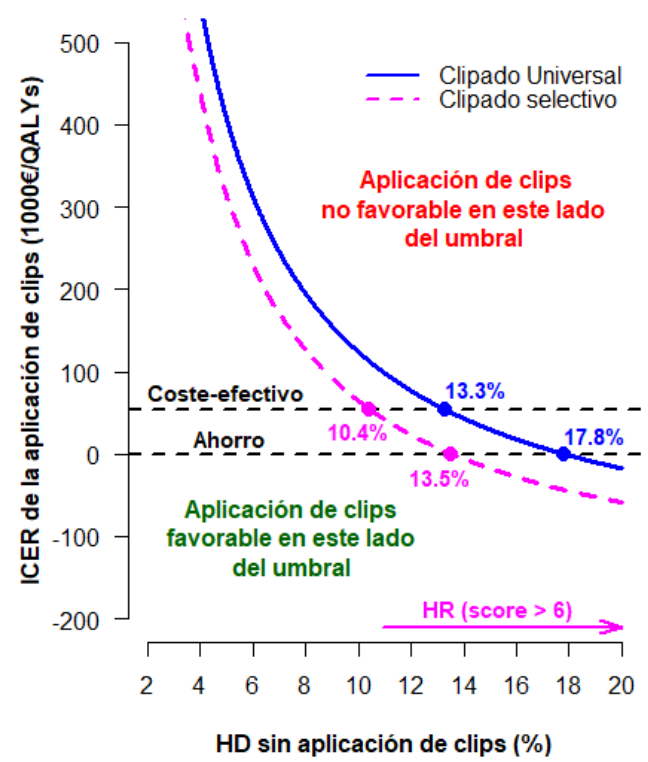

B) Contexto Americano

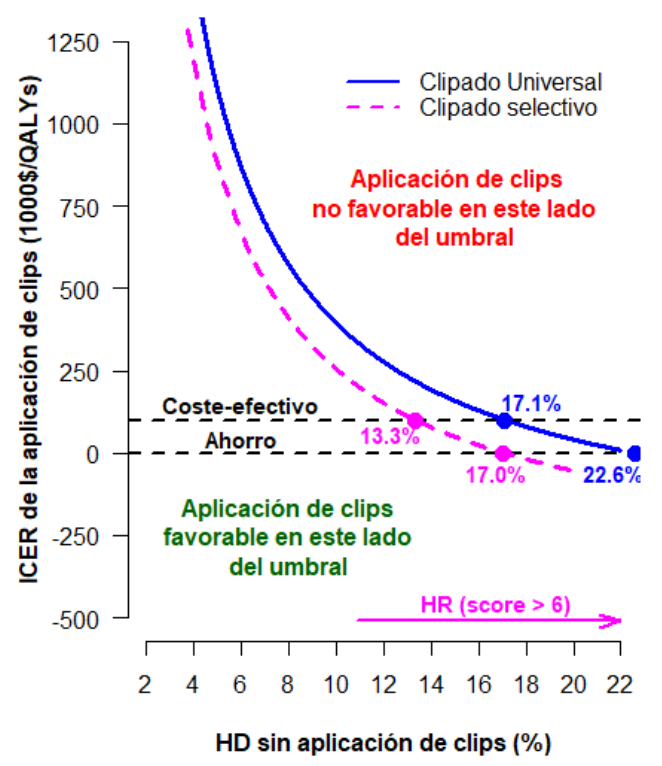

El análisis de sensibilidad bidireccional mostró que el CE del cierre del clip es sensible a la variación simultánea en la tasa de sangrado y otros parámetros como el coste de HD (Figura A5.3), el precio del clip (Fig.A5.4), la distancia entre clips (Fig.A5.5), el coste total del cierre (Fig. A5.6) y la reducción relativa del riesgo de HD tras aplicar clips (Fig. A5.7). 
Figura A5.3. Riesgo de HD vs coste de un episodio de HD para las estrategias de cierre universal y selectivo en los contextos económicos de Europa (A) y Norteamérica (B).

\section{A) Contexto Europeo}

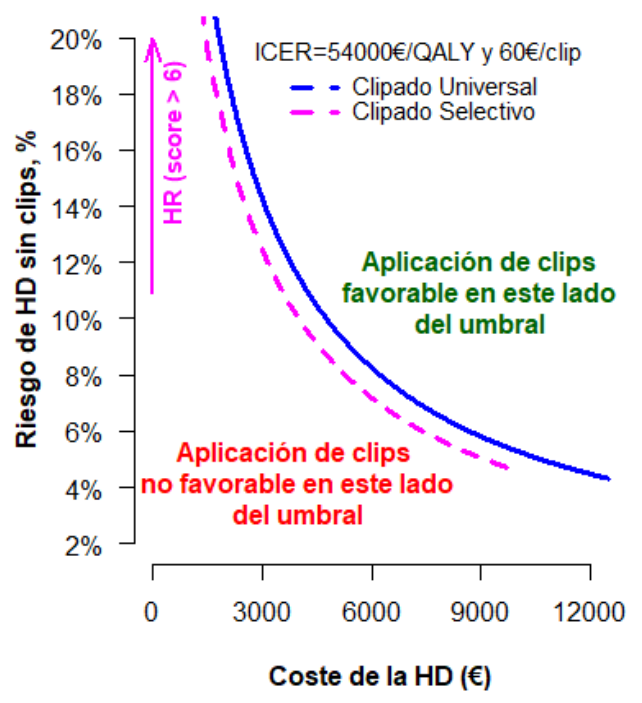

B) Contexto Americano

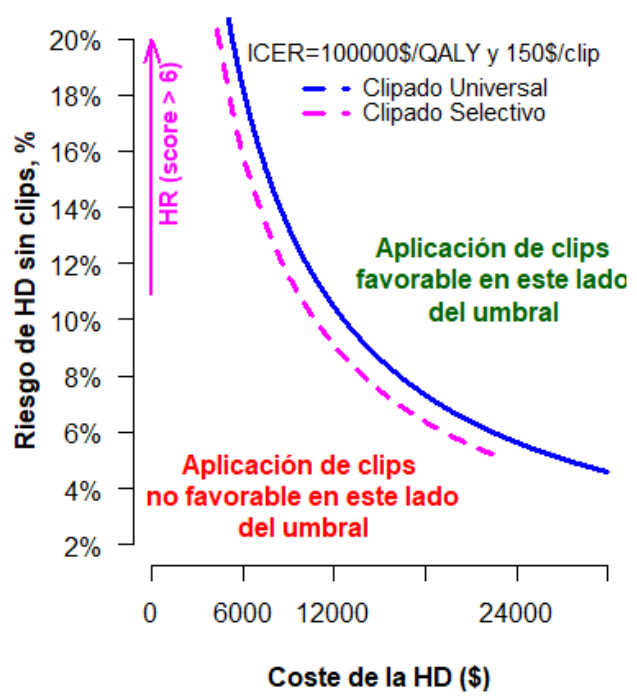

Figura A5.4. Riesgo de HD vs coste de un clip para las estrategias de cierre universal y selectivo en los contextos económicos de Europa (A) y Norteamérica (B).

A) Contexto Europeo

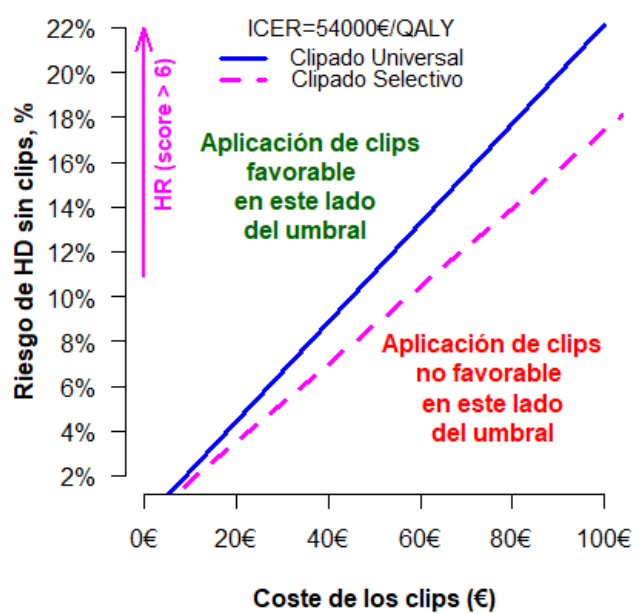

B) Contexto Americano

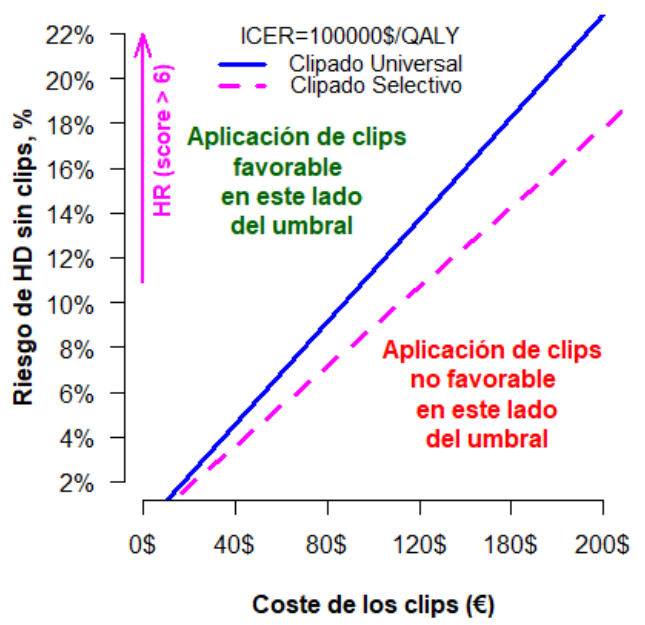


Figura A5.5. Riesgo de HD vs intervalo entre clips para las estrategias de cierre universal y selectivo en los contextos económicos de Europa $(A)$ y Norteamérica (B).

\section{A) Contexto Europeo}

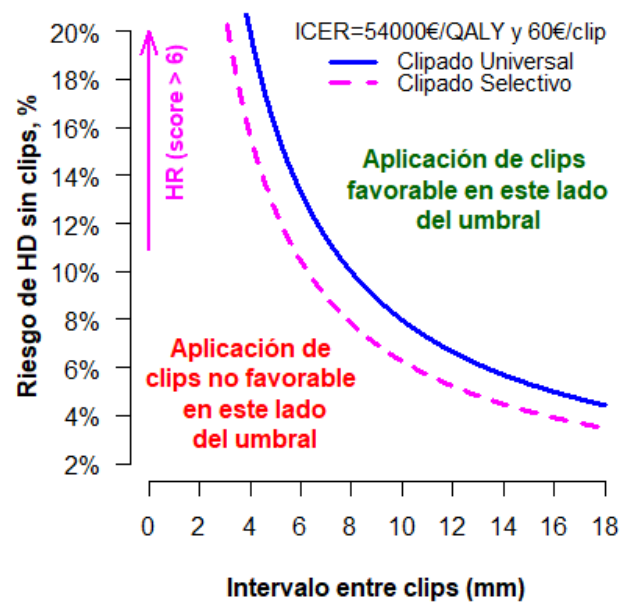

B) Contexto Americano

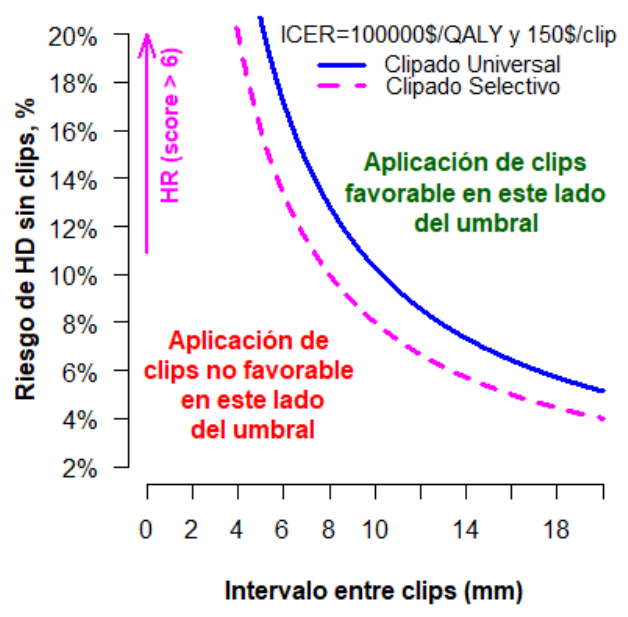

Figura A5.6. Riesgo de HD vs coste del cierre completo con clips para las estrategias de cierre universal y selectivo en los contextos económicos de Europa (A) y Norteamérica (B).

\section{A) Contexto Europeo}

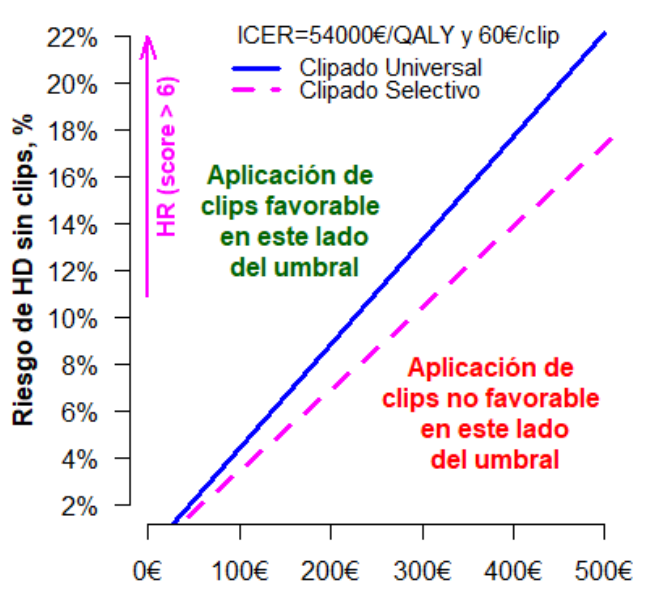

Coste total del cierre con clips/pólipo (€)

\section{B) Contexto Americano}

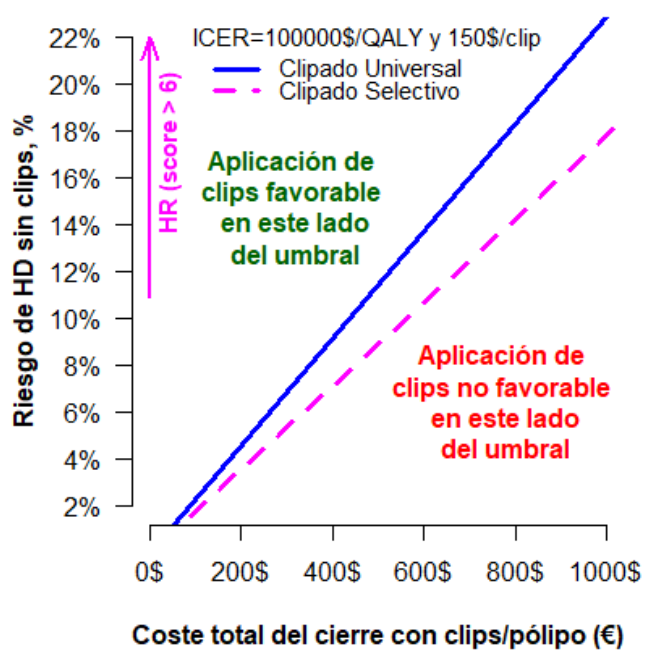


Figura A5.7. Riesgo de HD vs RRR de HD tras aplicar clips para las estrategias de cierre universal y selectivo en los contextos económicos de Europa (A) y Norteamérica (B).

A) Contexto Europeo

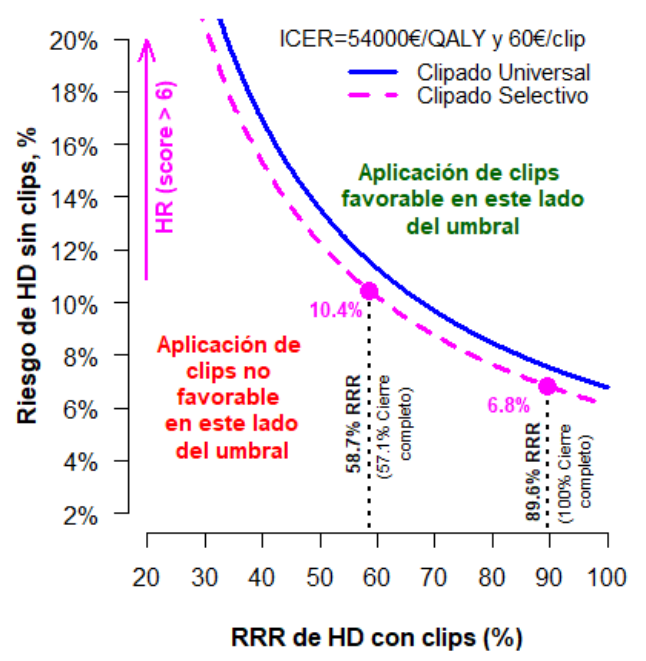

B) Contexto Americano

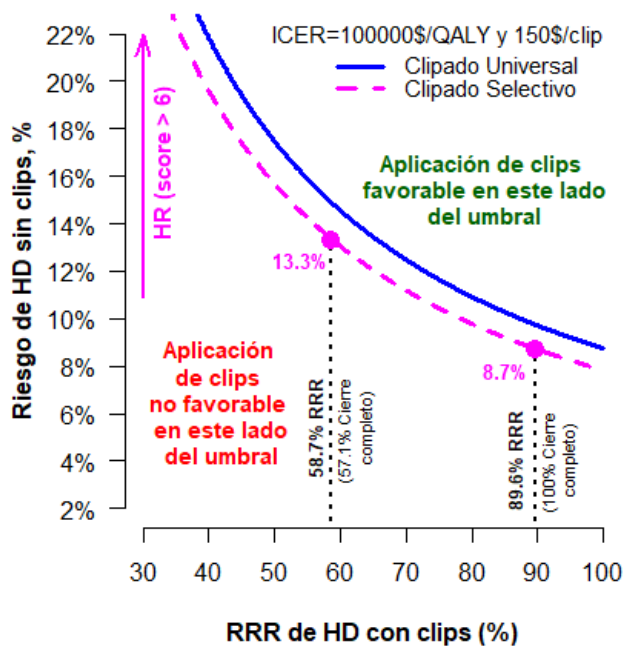

La fórmula matemática utilizada para nuestro análisis de $\mathrm{CE}$, que relaciona todos los parámetros antes mencionados, se muestra en la Fig. A5.8:

Figura A5.8. Fórmula matemática que relaciona todos los parámetros.

$$
I C E R=\frac{\left(\frac{\text { Tamaño } \times \text { Precioclip }}{\text { IntervaloClips }}\right)-R H D \times R R R \times \text { costeHD }}{R H D \times R R R \times P C V}
$$

ICER: El ratio del incremento CE; Tamaño: Tamaño medio de los pólipos de la cohorte; PrecioClip: Precio de un clip; IntervaloClips; Distancia entre clips usada en el cierre; RHD: Riesgo de HD sin aplicar clips; RRR: Reducción del riesgo relativo de HD tras aplicar clips; costeHD: Coste de un episodio de HD; PCV: Pérdida de calidad de vida tras una HD.

Los resultados del análisis de umbral para los 8 escenarios considerados en el análisis de sensibilidad se proporcionan en la Tabla A5.3. 
Tabla A5.3. Análisis del umbral de coste-efectividad.

\begin{tabular}{|c|c|c|c|c|}
\hline & \multicolumn{2}{|r|}{ Estrategia de cierre: } & \multirow{2}{*}{$\begin{array}{c}\text { Universal } \\
\mathbf{5 1 . 0 \%}\end{array}$} & \multirow{2}{*}{$\begin{array}{c}\text { Selectiva } \\
58.7 \%\end{array}$} \\
\hline & & RRR: & & \\
\hline $\begin{array}{l}\text { Parámetros umbrales } \\
\text { para CE o CS }\end{array}$ & Contexto & Umbral de ICER & & \\
\hline \multirow{2}{*}{$\begin{array}{l}\text { Tasa mínima de HD (no } \\
\text { cierre), para CE o CS, \% }\end{array}$} & EO & $\begin{array}{c}54.000 € / Q A L Y \\
0 € / Q A L Y\end{array}$ & $\begin{array}{l}13.3 \\
17.8\end{array}$ & $\begin{array}{l}10.4 \\
13.5\end{array}$ \\
\hline & NA & $\begin{array}{c}100.000 \$ / Q A L Y \\
0 \$ / Q A L Y\end{array}$ & $\begin{array}{l}15.3 \\
19.6\end{array}$ & $\begin{array}{l}13.3 \\
17.0\end{array}$ \\
\hline \multirow{2}{*}{$\begin{array}{l}\text { Coste mínimo de HD para } \\
\qquad \text { CE o CS, } €\end{array}$} & EO & $\begin{array}{c}54.000 € / Q A L Y \\
0 € / Q A L Y\end{array}$ & $\begin{array}{l}11,947 \\
13,072\end{array}$ & $\begin{array}{l}2,606 \\
3,730\end{array}$ \\
\hline & NA & $\begin{array}{c}100.000 \$ / Q A L Y \\
0 \$ / Q A L Y\end{array}$ & $\begin{array}{l}30,598 \\
32,680\end{array}$ & $\begin{array}{l}7,244 \\
9,326\end{array}$ \\
\hline \multirow{2}{*}{$\begin{array}{c}\text { Coste máximo de un clip } \\
\text { para CE o CS, } €\end{array}$} & EO & $\begin{array}{c}54.000 € / Q A L Y \\
0 € / Q A L Y\end{array}$ & $\begin{array}{l}20.3 \\
15.1\end{array}$ & $\begin{array}{l}78.8 \\
53.1\end{array}$ \\
\hline & NA & $\begin{array}{c}100.000 \$ / Q A L Y \\
0 \$ / Q A L Y\end{array}$ & $\begin{array}{l}44.0 \\
34.4\end{array}$ & $\begin{array}{l}154.1 \\
120.6\end{array}$ \\
\hline \multirow{2}{*}{$\begin{array}{l}\text { Coste máximo cierre } \\
\text { complete para } \\
\text { CE o CS, } €\end{array}$} & EO & $\begin{array}{c}54.000 € / Q A L Y \\
0 € / Q A L Y\end{array}$ & $\begin{array}{l}101.6 \\
75.8\end{array}$ & $\begin{array}{l}394.1 \\
265.5\end{array}$ \\
\hline & NA & $\begin{array}{c}100.000 \$ / Q A L Y \\
0 \$ / Q A L Y\end{array}$ & $\begin{array}{l}220.0 \\
172.0\end{array}$ & $\begin{array}{l}770.6 \\
603.0\end{array}$ \\
\hline \multirow{2}{*}{$\begin{array}{l}\text { Intervalo mínimo entre } \\
\text { clips para } \\
\text { CE o CS, mm }\end{array}$} & EO & $\begin{array}{c}54.000 € / Q A L Y \\
0 € / Q A L Y\end{array}$ & $\begin{array}{l}17.7 \\
23.7\end{array}$ & $\begin{array}{l}4.6 \\
6.8\end{array}$ \\
\hline & NA & $\begin{array}{c}100.000 \$ / Q A L Y \\
0 \$ / Q A L Y\end{array}$ & $\begin{array}{l}20.4 \\
26.1\end{array}$ & $\begin{array}{l}5.8 \\
7.5\end{array}$ \\
\hline
\end{tabular}

EO: Europa Occidental; NA: América del Norte. QALY: año de vida ajustado por calidad. ICER: El ratio del incremento coste-efectividad. CE: coste efectividad; CS: Estrategia de ahorro de costes (ICER $=0 € / Q A L Y)$ 
En nuestro entorno, para que el cierre universal sea CE, el coste directo de un evento de HD debería ser de al menos $11.947 €$, mientras que para el cierre selectivo debería ser de al menos $2606 €$. Del mismo modo, para que el cierre universal sea CE un clip debería costar $20 €$ o menos, mientras que para el cierre selectivo un clip podría costar hasta $79 €$. El coste total del cierre de una escara no debe superar los $102 €$ para la estrategia universal y los $394 €$ para la estrategia selectiva. Finalmente, se estimó que el intervalo mínimo entre clips para alcanzar la CE era de $17,7 \mathrm{~mm}$ para el cierre universal, pero podría reducirse a 4,6 $\mathrm{mm}$ para el cierre selectivo. En el entorno de América del Norte, para que el cierre universal sea CE, el coste de la HD debe ser de al menos 30.598 \$ o un clip debe costar 44 \$ o menos, mientras que para el cierre selectivo los umbrales para CE son 7.244 y $154 \$$ respectivamente.

\section{A5.4 CONCLUSIONES}

El cierre completo de los defectos mucosos post-resección en LCRNP es una medida CE cuando se aplica en pacientes con riesgo alto de sangrado, pero no es CE si se utiliza de forma universal después de cualquier RME.

Estos hallazgos sirven para contextos económicos muy diferentes, como podría ser el de Norte América (USA) y el de Europa Occidental (España).

En Europa Occidental el cierre profiláctico en los pacientes de alto riesgo de HD no sólo es CE sino que además ahorra costes (CS). 
Los análisis realizados permiten obtener para cada contexto económico, los riesgos mínimos de hemorragia, el precio máximo de los clips, el intervalo máximo entre clips, el coste máximo del cierre completo y el coste máximo de la HD para que la medida sea CE.

\section{A5.5 PUBLICACIÓN DEL TRABAJO}

Este trabajo fue aceptado como comunicación oral en la DDW, Chicago 2020 y publicado en forma de abstract en Gastrointestinal Endoscopy (GIE 2020,

91 6S: AB107). El estudio ha sido aceptado para publicación en la revista American Journal of Gastroenterology (FI 2019: 10.171). 


\section{DISCUSIÓN}

La RME es el tratamiento estándar para las LCRNP en los países occidentales $^{1}$. El evento adverso grave más común después de una RME es la HD, con una incidencia del $2,6-9,7 \%$ en lesiones de gran tamaño ( $\geq 2 \mathrm{~cm}$ ). Esta complicación conlleva una notable morbilidad con sus consiguientes costes asociados y con probabilidad de sufrir hospitalización, trasfusiones o incluso otros tratamientos como la embolización radiológica o la cirugía urgente $44,71,113,131,73,76-82$.

En diversos estudios observacionales se han descrito como factores de riesgo de HD post-resección la localización en colon proximal, el mayor tamaño de la lesión, la hemorragia intraprocedimiento, el uso de unidades electroquirúrgicas no controladas por microprocesador, la mayor edad del paciente, la hipertensión arterial, la toma de ácido acetil salicílico o anticoagulantes y la comorbilidad, en ocasiones medida por la clasificación anestésica $A S A^{44,76-82,113}$. Aunque los dos primeros factores de riesgo están más $\operatorname{aceptados}^{54,73,76,78,79,132-134}$, existe controversia en el papel del resto $44,45,73,76,78,134-136$.

En nuestro trabajo preliminar (Artículo 1 de la tesis $)^{112}$, que se basa en una cohorte prospectiva de 1255 EMR de LCRNP de gran tamaño, los factores asociados con HD fueron la edad del paciente $\geq 75$ años, la clasificación anestésica ASA III o IV, el uso de aspirina durante la RME, la ubicación de la lesión 


\section{Discusión}

proximal al colon transverso, un tamaño de la lesión $\geq 40 \mathrm{~mm}$ y la ausencia de cierre completo de la mucosa con clips.

Metz et al. también encontraron que la edad era un factor de riesgo de HD (OR por década de edad 1,70$)^{78}$. Aunque éste es un hallazgo importante debido a que la edad media de la población está aumentando y las complicaciones en pacientes mayores tienen un mayor impacto clínico podría tratarse de un factor asociado a otros como la comorbilidad, el mayor uso de tratamientos antiagregantes o anticoagulantes y a la presencia de lesiones de mayor tamaño. En nuestra serie el 30,6\% de los pacientes tenían más de 75 años. En cuanto a la comorbilidad asociada del paciente, plasmada como la clasificación anestésica ASA, un trabajo del grupo australiano refrendó esta asociación en una serie con 2128 pacientes $^{113}$.

Dos estudios con alto número de participantes encontraron que el uso de aspirina se asoció con una mayor prevalencia de hemorragia pospolipectomía ${ }^{78,136}$. En nuestra serie el riesgo de HD en los $50(4,1 \%)$ pacientes que recibieron tratamiento con aspirina durante la RME fue del $12 \%$. Sin embargo, otros estudios observacionales con tamaños muestrales altos no encontraron que el tratamiento con aspirina fuera un factor de riesgo $80,135,137$. Es importante resaltar que la aspirina puede suspenderse antes de la RME en pacientes que no tienen un alto riesgo de padecer eventos trombóticos. Si los pacientes tienen un alto riesgo se podrían considerar otras opciones profilácticas. Esta recomendación ya estaba respaldada por un análisis de costeeficacia que mostró que la estrategia de cierre profiláctico con clips para

prevenir la HD sólo era CE en pacientes en tratamiento antiagregante o 
anticoagulante $\mathrm{e}^{122}$.

La localización proximal como factor de riesgo ha sido destacada previamente en un ensayo controlado aleatoriado de 347 RME de grandes LCRNP y en un gran estudio observacional de 1172 pacientes ${ }^{44,81}$. Este ha sido el factor de riesgo con mayor influencia en el riesgo de HD en nuestro estudio. Otro factor que parece estar claramente asociado con la HD en nuestro estudio y en la literatura es el tamaño de la lesión ${ }^{73,76,80,94,138}$. Las lesiones más grandes probablemente tengan una vascularización más densa y el área de defecto mucoso post-resección es de mayor tamaño, lo que podría exponer estos vasos aumentando el riesgo de HD. Aunque no todos los estudios encontraron una asociación entre el tamaño de los pólipos y el sangrado ${ }^{44,139}$, la mayoría si lo hicieron. Como ejemplo destacamos un estudio retrospectivo de casos y controles que describió un aumento en el riesgo de HD post-polipectomía del 13\% por cada aumento de $1 \mathrm{~mm}$ en el diámetro del pólipo ${ }^{132}$.

La evidencia disponible en la actualidad en cuanto al cierre profiláctico con hemoclips del defecto mucoso es muy controvertida. En un amplio estudio retrospectivo de 524 LCRNP grandes el $47,1 \%$ de las lesiones no fueron cerradas con clips, el 9,9\% se cerró parcialmente y el 42,9\% recibió un cierre completo de la escara. El riesgo de HD fue del $9,7 \%$ en el grupo sin cierre frente al $1,8 \%$ en el grupo de cierre completo (OR 6,0; IC del 95\%: 2,0-18,5) $)^{79}$. El ensayo clínico randomizado de Zhang et al. asignó al azar el cierre de escaras o no a pacientes con RME o DSE de LCRNP mayores obteniendo un riesgo de HD del 1,1\% en el grupo de tratamiento vs $6,9 \%$ en el grupo control $(p<0,01)^{102}$. Sin embargo, el cierre con clips no previno la HD post-resección en lesiones con un tamaño 


\section{Discusión}

medio menor a $10 \mathrm{~mm}$ en otro ensayo controlado aleatorizado ${ }^{98}$. Parece posible que el pequeño tamaño de las lesiones y su menor riesgo de HD subestimaran el efecto preventivo de los clips.

Con los factores de riesgo asociados a la presencia de HD post-resección hemos desarrollado por primera vez un sistema de puntuación para estimar dicho riesgo. Este sistema de puntuación o score se denominará posteriormente score GSEED-RE (sistema de puntuación para HD diferida post-RME del grupo de resección de la Sociedad Española de Endoscopia Diferida) ${ }^{112}$. En orden de importancia los factores de riesgo que componen en modelo predictivo son los siguientes: localización proximal ( 3 puntos), cierre completo y uso de aspirina ( 2 puntos), tamaño, clasificación ASA y grupo de edad (1 punto). Con este score los pacientes pueden ser clasificados en 3 categorías de riesgo de acuerdo a una escala numérica: bajo riesgo (puntuación $0-3$, riesgo medio de sangrado del 0.6\%), riesgo intermedio (puntuación $4-6$, riesgo medio de sangrado del $5.5 \%$ ) y riesgo alto (puntuación 7-10, riesgo medio de sangrado del 40\%).

Dentro de estos factores existen dos teóricamente modificables, el uso de aspirina y el cierre preventivo con hemoclips. El resto, aunque no modificables, son fácilmente reconocibles por los clínicos y los endoscopistas. La puntuación obtenida con la suma de estos factores puede ayudar en la toma de decisiones en cuanto a la aplicación de medidas profilácticas o la observación clínica más estrecha en el grupo de alto riesgo, aunque estas recomendaciones no habían sido probadas en el momento de la publicación.

Como limitaciones del estudio se podrían citar las siguientes: falta de estandarización de los procedimientos; el carácter observacional y no controlado 
del estudio; la baja incidencia de hemorragia diferida; el menor número de sujetos en el subgrupo de alto riesgo, lo que puede explicar la sobreestimación del riesgo de HD en este subgrupo de población y la falta de cohorte externa de validación.

Como contrapartida cabe destacar el alto número de pacientes reclutados, que el trabajo se realizó de forma multicéntrica por endoscopistas experimentados con requisitos mínimos anuales de inclusión y que como método alternativo a la validación externa se utilizó una validación interna booststrap para modelos logísticos. Los parámetros estadísticos cumplieron con los estándares aceptados: ROC del modelo multivariado y del score de 0.79 (IC95\% 0.72-0.85); test de Hosmer-Lemeshow no significativo $(X 2=5,72 ; p=0,572)$ y pendiente de discriminación (diferencia en las predicciones promedio para aquellos con y sin el resultado) de 0,07 , con un IC del 95\% (0.04- 0.1; $p<.001)$.

De forma prácticamente simultánea con la publicación definitiva del trabajo anterior ${ }^{112}$, el grupo australiano del profesor Bourke publicó otro modelo llamado ACER risk score ${ }^{113}$. La localización proximal (2 puntos), el tamaño de la lesión ( 2 puntos), la presencia de comorbilidad mayor (1 punto) y la ausencia de epinefrina en la solución de inyección (1 punto) formaban un sistema de puntuación del 0 al 6 dividido en 3 categorías de riesgo (bajo o score 0-1, medio o score 2-4 y alto o score 5-6). Las probabilidades medias de HD asociadas a dichas categorías fueron del 3.4\%, 6,2\% y del 15.7\% en la cohorte de validación.

Aunque ambas series incluyeron un gran número de pacientes, las variables independientes resultantes de estos estudios no concordaban, y ningún 


\section{Discusión}

modelo había sido validado externamente en cohortes diferentes a las utilizadas para su derivación.

En nuestro trabajo multicéntrico y prospectivo ${ }^{115}$, validamos nuestro modelo previo (score GSEED-RE) y realizamos la primera validación externa del modelo ACER (Artículo 2 de la tesis). Para ello utilizamos 3 cohortes, nuestra serie de pacientes Pre-2015, la serie Post-2015 y la cohorte total.

El rendimiento de los modelos GSEED-RE y ACER originales fue discreto. Sin embargo, los modelos recalibrados y reestimados de ambos lograron un mejor rendimiento, pero todavía subóptimo. Estos resultados sugerían que los modelos anteriores debían perfeccionarse antes de su uso recomendado en la práctica clínica. Ésto nos motivó a desarrollar otro modelo que pudiera ofrecer mejoras. Este modelo incluye cuatro variables fácilmente identificables que pueden evaluarse antes de realizar cualquier procedimiento: comorbilidad mayor (1 punto), tamaño mayor de $4 \mathrm{~cm}$ (2 puntos), uso de antiagregantes o anticoagulantes (3 puntos) y lesiones localizadas proximales al ángulo hepático (3 puntos). Las probabilidades medias de HD asociadas a las categorías de riesgo del modelo fueron: bajo riesgo (0-3 puntos) $2.2 \%$, riesgo medio (4-6 puntos), $4.1 \%$ y riesgo alto (7-9 puntos), $14.8 \%$.

Aunque el rendimiento no aumentó mucho en comparación con modelos anteriores el modelo elimina ciertas variables que no eran consistentemente significativas en las diferentes cohortes.

Como limitaciones del estudio, destacan: la variabilidad entre endoscopistas en cuanto a su experiencia, formación y variabilidad en su técnica, un posible sesgo de selección de casos debido a que no se han 
contabilizado todos los casos que han sido remitidos para tratamiento quirúrgico, la heterogeneidad de la variable comorbilidad, ya que se incluyen comorbilidades mayores que pueden tener muy diferente impacto en la salud del paciente, la baja frecuencia del evento (HD) y la ausencia de datos suficientes para poder categorizar la variable antiagregación/anticoagulación en 3 categorías. Como fortalezas del mismo destacamos el alto número de pacientes incluidos y la participación de múltiples endoscopistas e instituciones. Es necesario que otros grupos externos validen nuestro modelo y que prueben el impacto del número de categorías de antiagregantes y anticoagulantes en el rendimiento del modelo.

Las ventajas del nuevo score son las siguientes:

- ROC aceptables en todas las cohortes (AUC 0,69-0,74), lo que sugiere una mayor estabilidad en comparación con propuestas anteriores y una menor susceptibilidad a cambios en los conjuntos de datos.

- Los gráficos de calibración también fueron más estables en las cohortes y las versiones del modelo.

- La pendiente de discriminación para el nuevo modelo fue mayor y más consistente entre las cohortes que las propuestas previas, lo que sugiere una mayor capacidad de discriminación y consistencia en la predicción de HD.

-La probabilidad de HD en la categoría de alto riesgo se mantuvo estable en todas las cohortes y fue más alta que el modelo ACER independientemente de la cohorte $(13,4 \%$ y $17,1 \%$ para el nuevo modelo frente a $10,0 \%$ y $8,9 \%$ para el modelo ACER, en las cohortes pre 2015 y post 2015 respectivamente), lo que muestra una mejor detección de pacientes de alto riesgo. 


\section{Discusión}

- Las variables pueden identificarse con facilidad antes del procedimiento y se han eliminado variables relacionadas con medidas preventivas como el cierre con clips, ya que el objetivo principal de este score fue diseñar una herramienta que ayude al clínico a clasificar de antemano a los pacientes por su riesgo de HD y diseñar "a priori" la mejor estrategia profiláctica según dicho riesgo.

- El modelo predice el riesgo de HD en 10 grupos (score 0-9) dividido en 3 categorías (riesgo bajo, medio y alto), siendo la principal utilidad del modelo la identificación de los pacientes con alto riesgo de hemorragia.

- Sólo el $10 \%$ de los pacientes están en la categoría de alto riesgo. Dado que los recursos clínicos son limitados este modelo permite una mejor selección de pacientes que pueden beneficiarse más de las medidas preventivas como el cierre de los defectos mucosos o un manejo más personalizado de la antiagregación o anticoagulación. Los modelos previos no incluían la toma de anticoagulantes y el modelo australiano no incluía tampoco la toma de antiagregantes. La importancia de tener en cuenta el tratamiento anticoagulante como factor de riesgo va a ser expuesto en un próximo metaanálisis en el que participamos con nuestros datos crudos (Turan, Siersema et al, manuscrito en fase de escritura).

- El score también puede ser útil en cuestiones logísticas como la necesidad de apoyo familiar o de permanecer cerca del hospital al menos durante la primera semana en los pacientes de alto riesgo; por el contrario, nos aporta una gran dosis de tranquilidad en los pacientes de bajo riesgo donde no estarían indicadas la aplicación de medidas profilácticas, lo que supone un notable ahorro económico. 
- Finalmente, el nuevo modelo puede mejorar la selección de candidatos para su inclusión en ensayos clínicos, con lo que se asegura la obtención de datos más fiables y la reducción del tamaño muestral. La ventaja de utilizar un score de riesgo ha sido demostrada en nuestro ensayo clínico sobre cierre de defectos mucosos post-resección.

Tras indagar sobre los factores de riesgo asociados a la HD post-resección y agruparlos en un sencillo modelo predictivo, de forma paralela nuestro interés se centró en estudiar mediante un ensayo clínico aleatorizado la medida con mayor potencial de eficacia en la prevención de este evento adverso, el cierre completo con hemoclips (Artículo 3 de la tesis).

Un gran estudio retrospectivo que incluyó solo LCRNP de gran tamaño mostró una tasa de HD del $9.7 \%$ en el grupo sin cierre preventivo y del 1,8\% en el grupo de cierre completo con $\operatorname{clips}^{79}$. A pesar de las limitaciones de este estudio no controlado se observó un claro beneficio del cierre con clips especialmente cuando el cierre del clip fue completo.

Varios ensayos clínicos randomizados han analizado esta medida ${ }^{98-102}$. Un metanálisis de esos ensayos no mostró ningún beneficio del cierre preventivo, con un riesgo de $\mathrm{HD}$ del $2,2 \%$ en el grupo de tratamiento frente al $2,7 \%$ en el grupo control ${ }^{103}$. Sin embargo, ese metaanálisis y los ensayos clínicos que lo componen no fueron diseñados específicamente para evaluar la HD en LCRNP de gran tamaño y presentaban varias limitaciones como el bajo riesgo de HD en lesiones pequeñas, la morfología heterogénea de los pólipos, la definición 


\section{Discusión}

imprecisa de HD y la diferencia en las técnicas de resección utilizadas ${ }^{102}$.

En 2019 el grupo de Pohl et al. publicó los resultados de un ensayo clínico aleatorizado con gran número de pacientes ( $\mathrm{n}=928$ pacientes), mostrando un beneficio del cierre profiláctico para cualquier LCRNP de gran tamaño ${ }^{119}$. Aunque la población incluida era muy numerosa, algunos pólipos (como los pólipos distales) tenían un riesgo bajo de HD, lo que resultaba en un NNT alto (28). La localización proximal, el gran tamaño, la presencia de comorbilidades importantes $y$ el tratamiento antiagregante $\mathrm{o}$ anticoagulante han sido reconocidos previamente como factores de riesgo independientes de $H D^{112,113}$. La localización proximal fue la variable con mayor peso en la puntuación del GSEED-RE. En el ensayo de Pohl et al. la localización proximal fue la única variable que demostró un efecto estadísticamente significativo en el grupo de cierre con clips ${ }^{119}$. A pesar de que hubo un mayor riesgo de HD con un mayor tamaño de pólipo y en pacientes con medicación antiplaquetaria, el estudio no mostró un beneficio del cierre con clips en esos pacientes. Además, no se realizó ninguna evaluación del riesgo de HD antes de la inclusión y en consecuencia, el riesgo de HD de la población del ensayo dependió de la proporción relativa de casos de alto y bajo riesgo.

En nuestro ensayo clínico se incluyeron solamente lesiones con riesgo sustancial de HD, con un riesgo mínimo esperado de HD mayor o igual al 8\%. Más del $90 \%$ de los casos eran pólipos proximales y el $51 \%$ eran mayores de $40 \mathrm{~mm}$; además, el $36 \%$ de los pacientes estaban tomando antiagregantes plaquetarios. Combinamos esas variables utilizando la puntuación GSEED-RE, lo que resultó en una tasa de HD del 12,1\% en el grupo de control, que fue mucho más alta que la 
informada en otros estudios.

Nuestros resultados mostraron una clara tendencia de reducción de HD en el análisis ITT, con una diferencia absoluta del riesgo del $7 \%(p=0,053)$. La asociación alcanzó significación estadística tras ajustar por la variable de tratamiento antiagregante (más frecuente en el grupo de clips). El efecto protector del cierre con clips fue más evidente cuando se logró un cierre completo, con una diferencia absoluta del riesgo del $10,6 \%$.

Merece especial atención el análisis por subgrupos en función de la localización de los pólipos. Se consideraron dos definiciones de ubicación proximal, una que incluía el colon transverso y otra que excluía este segmento colónico. Con ambas definiciones los resultados fueron similares. Además, la incidencia de HD en el colon transverso fue muy baja; entre los 35 pólipos de colon transverso, solo hubo 1 caso de HD en el grupo de intervención (un cierre fallido) y ningún caso en el grupo de control. En el subgrupo de pólipos proximales hubo una tendencia de un efecto protector del cierre del clips y la asociación fue estadísticamente significativa cuando se completó el cierre del clips. Los pólipos distales estuvieron infrarrepresentados y no se pudieron extraer conclusiones sólidas al respecto.

El ensayo tuvo las siguientes limitaciones: el criterio de selección (score GSEED-RE) no ha sido validado externamente en otros contextos aunque proviene de una amplia serie prospectiva; la incidencia de HD en nuestra población seleccionada fue difícil de predecir y afectó el cálculo del tamaño de la muestra; el momento de la aleatorización es un tema controvertido, la aleatorización después de completar la RME pudo evitar una posible aplicación 


\section{Discusión}

más intensiva de otras medidas preventivas de HD en el grupo de control pero pudo introducir un sesgo de selección hacia la exclusión de defectos mucosos difíciles de cerrar con clips; no se recogió la información de los pólipos excluidos para el estudio; el cierre completo no fue posible en el $43 \%$ de los casos ya que las lesiones eran muy grandes (más de la mitad $\geq 40 \mathrm{~mm}$ ) y se utilizaron exclusivamente hemoclips de $11 \mathrm{~mm}$, quedando excluidas otras posibilidades terapéuticas como el uso de clips de mayor tamaño u otras técnicas de cierre, sin embargo fue necesaria la introducción de un criterio homogéneo para asegurar la validez del ensayo.

Dentro de las ventajas de nuestro ensayo además de la inclusión de pacientes con riesgo substancial de HD podemos citar las siguientes: el carácter multicéntrico del mismo; sólo se incluyeron pacientes con RME y no otras técnicas; todas las lesiones fueron de gran tamaño; se evitaron en lo posible factores de confusión con otras medidas profilácticas; no hubo tratamientos cruzados entre los grupos y sólo se incluyó un pólipo por paciente.

La evidencia que apoya el cierre profiláctico con hemoclips para prevenir la HD post-resección ha sido controvertida hasta el año 2019. Como ejemplo, la Sociedad Europea de Endoscopia Digestiva (ESGE) sugería que esta medida podía tener un papel, individualizando la decisión en función de los factores de riesgo del paciente. Sin embargo, esta recomendación ha sido calificada como débil por estar basada en evidencias de calidad subóptima².

Metaanálisis previos, principalmente basados en estudios que incluían 
lesiones de pequeño tamaño $(<20 \mathrm{~mm})$ y con alto riesgo de sesgo no informaron acerca de ningún efecto protector del cierre profiláctico post-resección ${ }^{103,140,141}$.

En 2019 se publican en Gastroenterology 3 ensayos clínicos al respecto que se describen brevemente a continuación ${ }^{118-120}$. El trabajo de Feagins et al ${ }^{118}$ es un estudio de equivalencia con dos ramas. Incluyó 1098 pólipos mayores de $1 \mathrm{~cm}$ denominándolos de gran tamaño, dentro de los cuales solo el 39\% ( $n=222)$ era mayor de dos centímetros y donde además se mezclaban pólipos pediculados con no pediculados cuya fisiopatología de sangrado es notablemente diferente. El estudio se terminó con 1050 pacientes y no 1622 por falta de fondos. No encontraron diferencias significativas en el riesgo de HD del grupo control (2.9\%) con respecto a la del grupo de tratamiento (2.3\%) (RR=0.79, IC95\% 0.37-1.66).

El ensayo clínico de Pohl et $a^{119}$, randomizó 928 pacientes con una adecuada metodología. Se produjo HD en el 3,5\% de los pacientes en el grupo de clips y en el 7,1\% del grupo control (ARD, 3,6\%; IC del 95\%, 0,7\% -6,5\%). Entre 615 pacientes $(66,9 \%)$ con pólipos localizados en el colon proximal el riesgo de sangrado en el grupo de clips fue del 3,3\% y en el grupo control fue del 9,6\% (ARD, 6,3\%; IC del 95\%, 2,5\% -10,1\%). Con respecto a los pólipos del colon distal, los riesgos fueron del $4,0 \%$ en el grupo de clips y del $1,4 \%$ en el grupo control (ARD, $-2,6 \%$; IC del $95 \%,-6,3 \%$ a $-1,1 \%$ ). El efecto del cierre del clip fue independiente de la medicación anticoagulante o del tamaño del pólipo.

Debido a las nuevas evidencias disponibles se realizó una revisión sistemática y un metaanálisis de todos los ensayos clínicos disponibles con el objetivo primario de aclarar el papel profiláctico de los hemoclips en la prevención de la HD tras la resección endoscópica de lesiones colorrectales 


\section{Discusión}

\section{(Artículo 4 de la tesis).}

Según nuestro metaanálisis el cierre preventivo con clips no reduce el riesgo de HD post-resección de manera global, principalmente debido al riesgo muy bajo de HD (3.3\%) en el grupo de control. Sin embargo, cuando se aplicó a lesiones con mayor riesgo de HD, como aquellas $\geq 20 \mathrm{~mm}$ y de localización proximal, redujo aproximadamente un $50 \%$ el riesgo de HD.

Estos resultados son relevantes por las siguientes razones. En primer lugar, el trabajo tuvo la suficiente potencia estadística para poder excluir la falta de eficacia en las lesiones de pequeño tamaño. En segundo lugar, se excluyó la heterogeneidad significativa entre los estudios así como el sesgo de publicación. Esto indicaba que el efecto es homogéneo en las diferentes series, lo que aseguraba la reproducibilidad de los resultados. En tercer lugar, la magnitud de población incluida, más de 7.000 lesiones aleatorizadas, nos permitió realizar dos estratificaciones de riesgo principales. Nuestras estimaciones ajustadas atribuyeron un aumento de casi el doble (del 4,3\% al 7,6\%) en el riesgo de HD cuando las lesiones eran $\geq 20 \mathrm{~mm}$. Con respecto a la localización, los datos también respaldan la localización proximal como factor de riesgo para HD y la eficacia del cierre preventivo. Se observó un aumento de casi el doble en el riesgo de $\mathrm{HD}$ cuando no se cerraba con clips, del 3,3\% en el colon distal al 6,2\% en el colon proximal. Respecto a la interacción entre tamaño y ubicación, el tamaño $\geq 20 \mathrm{~mm}$ parecía ser el principal determinante del riesgo de $\mathrm{HD}$. El beneficio del cierre preventivo fue claro en grandes lesiones proximales, pero estaba ausente para las lesiones pequeñas, incluso cuando estaban localizadas en el colon proximal. Por otro lado, no hubo diferencias significativas que 
apoyaran el cierre preventivo de lesiones de gran tamaño distales.

Las limitaciones del estudio son las siguientes: la falta de datos para valorar los efectos de la medicación anticoagulante; varios estudios son de moderada calidad metodológica; la inclusión de pacientes sometidos a múltiples resecciones y la ausencia de información acerca de algunos datos técnicos que han podido afectar al riesgo de HD y que no han sido debidamente recogidos.

Nuestro datos concuerdan con un análisis de CE reciente que utiliza los costes de Medicare (USA) y que se centra en el impacto de la adopción del cierre rutinario con clips desde una perspectiva del pagador ${ }^{124}$. Teniendo en cuenta que los clips son caros y su colocación puede ser técnicamente exigente, el cierre preventivo debería ser reservado a pacientes con mayor riesgo de sufrir este evento adverso, lo que supondría una reducción paralela de complicaciones y costes. Nuestro trabajo apoya el uso preventivo de clips en cualquier lesión proximal de gran tamaño, excluyendo su uso para aquellas de pequeño tamaño y distales. Es probable que la opción de cerrar escaras distales de gran tamaño dependa de otros factores de riesgo asociados ${ }^{125}$.

A pesar de que la evidencia actual respalda el cierre preventivo con clips tras RME de LCRNP de gran tamaño proximales o cuando existe riesgo sustancial de sangrado, existen pocos trabajos y con resultados contradictorios en cuanto a su coste-efectividad.

Nuestro estudio (Artículo 5 de la tesis) muestra que el uso de clips hemostáticos para cerrar completamente el defecto mucoso post-resección en la 


\section{Discusión}

prevención de la HD es una medida CE cuando se aplica a pacientes con alto riesgo de HD según el score de puntuación GSEED-RE2, pero no cuando se aplica a todos los pacientes sometidos a RME de LNPCP.

Los primeros estudios de CE se basaron en datos probabilísticos y obtenidos de la literatura. Parikh et al. ${ }^{122}$ sugirieron que el cierre de un pólipo de 10-15 mm utilizando un solo clip sería CE exclusivamente en pacientes que reciben tratamiento antiagregante o anticoagulante. En contraste, Bahin et al. ${ }^{123}$ encontraron que el cierre preventivo con clips no era económicamente viable siguiendo una estrategia universal (cierre en todos los pólipos) o selectiva (pólipos proximales). Este estudio utilizó datos reales de una gran cohorte prospectiva; sin embargo, la estimación del beneficio clínico fue teórica y no se basó en un ensayo clínico. Este análisis arrojó resultados negativos, probablemente debido al bajo riesgo de HD de la población objetivo, la costosa profilaxis (clips caros y muchos clips necesarios por lesión) y el bajo coste del manejo de la HD. Recientemente se publicó un tercer estudio realizado para predecir los costes de la atención médica para manejar la HD después de un cierre profiláctico de rutina ${ }^{124}$. Basado en la estimación de los costes de HD para un individuo de 65 años con al menos una comorbilidad y dentro del sistema Medicare, el cierre universal con clips supuso un ahorro de costes pero sólo para los pólipos ubicados en el colon proximal. Aunque este estudio se centró en los costes y no consideró el gasto en clips, apoya un reembolso de la profilaxis con clips que actualmente no es ampliamente aceptado.

Nuestro estudio ${ }^{120}$ y el estudio de Shah et al. ${ }^{124}$ difieren en los criterios utilizados para seleccionar los pólipos que deben cerrarse: pólipos de localización 
proximal ${ }^{124}$ o pólipos dentro de la categoría de alto riesgo según la puntuación GSEED-RE2 ${ }^{120}$, lo que significa que nuestra selección implica también la presencia de medicación anticoagulante o antiplaquetaria, así como al menos otro factor de riesgo (comorbilidad mayor o gran tamaño de la lesión ( $\geq 40 \mathrm{~mm})$ ). La proporción de pólipos que deben cerrarse también difiere; es decir, si se utiliza la ubicación proximal como criterio se necesitaría cerrar el $52 \%$ de todas las lesiones grandes y la selección de pacientes con un alto riesgo de HD resultaría en un cierre de únicamente el $12 \%$ de todas las lesiones.

La selección de pacientes con alto riesgo de HD supondría un ahorro de 44 $€$ por pólipo en Europa Occidental y sería una estrategia CE tanto en Europa Occidental como en Norteamérica.

Nuestro análisis de sensibilidad se puede utilizar para evaluar otros escenarios clínicos y económicos. Por ejemplo, asumiendo los parámetros de Pohl et al. ${ }^{119}$ y el coste de manejo de la HD del estudio de Shah et al. ${ }^{124}$ que fue de $6,458 \$$, el cierre con clips de $150 \$$ podría ser CE para pacientes con riesgo de HD superior al $11,5 \%$. Teniendo en cuenta el riesgo real de HD de esa cohorte $(9,6 \%)$ y el mayor coste de HD, como los $9.500 \$$ del estudio de Parikh et al ${ }^{122}$, se podría alcanzar la CE incluso si el precio de un clip aumentara a $170 \$$.

También cabe mencionar que la RME es mucho más económica y menos invasiva, con menores tasas de morbilidad y mortalidad que la alternativa quirúrgica. El coste directo de una hemicolectomía sin complicaciones en nuestro contexto se ha estimado en $4226 €$ (en comparación con $1029 €$ para la RME de un pólipo de colon de gran tamaño) ${ }^{142}$. Incluso sin tener en cuenta los costes adicionales de las posibles complicaciones quirúrgicas, la diferencia de costes 


\section{Discusión}

entre la resección endoscópica y la alternativa quirúrgica es tan grande que el tratamiento endoluminal también puede considerarse un procedimiento que ahorra costes. Futuras innovaciones como clips más baratos, técnicas avanzadas de cierre ${ }^{143}$ o la aplicación de sustancias o geles que cubran el defecto mucoso ${ }^{110}$, pueden cambiar el escenario actual. El análisis de sensibilidad de nuestro estudio también es útil para proporcionar el marco para abordar tales cambios, teniendo en cuenta las variables correspondientes.

Las limitaciones del estudio se detallan a continuación. Primero, sólo se midieron los costes directos; no hay datos psicométricos de nuestra cohorte por lo que no pudimos estimar de forma individual las disminuciones de la utilidad después de un evento de HD; además, se asumió la misma disminución de utilidad en los diferentes grupos de riesgo.

Nuestro trabajo presenta las siguientes ventajas. Los parámetros utilizados han sido obtenidos de datos reales. En segundo lugar, al aplicar el score GSEEDRE2, identificamos lesiones con alto riesgo de HD aumentando la probabilidad de encontrar una estrategia CE. Finalmente, nuestro análisis de umbral junto con el análisis de sensibilidad bidireccional cubre una amplia gama de escenarios clínicos y económicos representando la práctica totalidad de las casuísticas que pueden ser válidas en diferentes países del mundo. 


\section{RESUMEN DE CONCLUSIONES GENERALES}

A1.1 Seis variables se asociaron con HD después de la RME de LCRNP de gran tamaño: edad $\geq 75$ años, clasificación ASA III o IV, uso de aspirina durante la RME, ubicación de la lesión proximal al colon transverso, tamaño de la lesión $\geq 40 \mathrm{~mm}$ y ausencia de cierre completo de la escara con clips.

A1.2 Se ha desarrollado un modelo predictivo con dichas variables, denominado GSEED-RE, que estima el riesgo de HD post-resección con una capacidad predictiva aceptable.

A2.1 La validación externa de los modelos GSEED-RE y ACER mostró resultados subóptimos.

A2.2 El nuevo modelo denominado GSEED-RE2, integrado por 4 variables fácilmente identificables antes de la RME (localización proximal de la lesión, uso de antiagregantes/anticoagulantes, tamaño $\geq 40 \mathrm{~mm} \mathrm{y}$ comorbilidad mayor), predice el riesgo de HD con una capacidad predictiva mayor que la de los dos modelos previos (GSEED-RE y ACER). 


\section{Conclusiones}

A3.1 El cierre de los defectos mucosos post-resección de LCRNP con riesgo sustancial de sangrado reduce el riesgo de HD. Este efecto es debido al cierre completo de las escaras.

A4.1 La práctica universal del cierre completo de los defectos mucosos post-resección no reduce el riesgo de HD. Sin embargo, el cierre fue eficaz en reducir el riesgo de HD cuando se utiliza de forma selectiva en las lesiones proximales de gran tamaño.

A5.1 El cierre completo de los defectos mucosos post-resección en LCRNP, es una medida CE cuando se aplica en pacientes con riesgo alto de sangrado, pero no lo es si se utiliza de forma universal después de cualquier RME.

A5.2 En Europa Occidental, el cierre profiláctico en los pacientes de alto riesgo de $\mathrm{HD}$ no sólo es $\mathrm{CE}$, sino que además ahorra costes (CS).

A5.3 La fórmula matemática que relaciona todos los parámetros de CE (ICER, tasa de sangrado, tamaño del pólipo, precio del clip, intervalo entre clips, coste de la HD, reducción relativa del riesgo de HD y pérdida de calidad de vida por episodio de sangrado) permite realizar un análisis personalizado para cada contexto económico. 


\section{CONCLUSIONES FINALES}

1. Cuatro variables identificables antes de la RME (localización proximal de la lesión, uso de antiagregantes/anticoagulantes, tamaño $\geq 40 \mathrm{~mm}$ y comorbilidad mayor) integradas en un modelo denominado GSEED-RE2 predicen el riesgo de HD post-resección de LCRNP de gran tamaño.

2. El cierre de los defectos mucosos post-resección de LCRNP con alto riesgo de sangrado reduce el riesgo de HD. Este efecto es debido al cierre completo de las escaras y se trata de una medida coste-efectiva en cualquier contexto económico cuando se aplica a pacientes de alto riesgo de HD. 
upna 


\section{REFERENCIAS}

1. Kantsevoy S V., Adler DG, Conway JD, et al. Endoscopic mucosal resection and endoscopic submucosal dissection. Gastrointest Endosc. 2008. doi:10.1016/j.gie.2008.01.037

2. Ferlitsch M, Moss A, Hassan $C$, et al. Colorectal polypectomy and endoscopic mucosal resection (EMR): European Society of Gastrointestinal Endoscopy (ESGE) Clinical Guideline. Endoscopy. 2017. doi:10.1055/s0043-102569

3. Tanaka S, Kashida H, Saito $Y$, et al. JGES guidelines for colorectal endoscopic submucosal dissection/endoscopic mucosal resection. Dig Endosc. 2015. doi:10.1111/den.12456

4. Rosenberg N, Brunswick N. Submucosal saline wheal as safety factor in fulguration of rectal and sigmoidal polypi. AMA Arch Surg. 1955. doi:10.1001/archsurg.1955.01270070122021

5. Deyhle $P$, Largiadèr $F$, Jenny $S$, Fumagalli I. A Method for Endoscopic Electroresection of Sessile Colonic Polyps. Endoscopy. 1973. doi:10.1055/s-0028-1098209

6. Tada $M$, Shimada $M$, Murakami $F$, et al. Development of the strip-off biopsy. Gastroenterol Endosc. 1984. doi:10.11280/gee1973b.26.833

7. Larghi A, Waxman I. State of the Art on Endoscopic Mucosal Resection and Endoscopic Submucosal Dissection. Gastrointest Endosc Clin N Am. 2007. doi:10.1016/j.giec.2007.05.012

8. Hurlstone DP, Sanders DS, Cross SS, et al. Colonoscopic resection of lateral spreading tumours: A prospective analysis of endoscopic mucosal resection. Gut. 2004. doi:10.1136/gut.2003.036913

9. The Paris endoscopic classification of superficial neoplastic lesions: Esophagus, stomach, and colon - November 30 to December 1, 2002. In: Gastrointestinal Endoscopy. ; 2003. doi:10.1016/S0016-5107(03)02159-X

10. Moss A, Bourke MJ, Williams SJ, et al. Endoscopic mucosal resection outcomes and prediction of submucosal cancer from advanced colonic 
Referencias

mucosal neoplasia. $\quad$ Gastroenterology. 2011. doi:10.1053/j.gastro.2011.02.062

11. Conio M, Ponchon T, Blanchi S, Filiberti R. Endoscopic mucosal resection. Am J Gastroenterol. 2006. doi:10.1111/j.1572-0241.2006.00424.x

12. Wong Kee Song LM, Adler DG, Chand B, et al. Chromoendoscopy. Gastrointest Endosc. 2007. doi:10.1016/j.gie.2007.05.029

13. Buchner AM, Guarner-Argente C, Ginsberg GG. Outcomes of EMR of defiant colorectal lesions directed to an endoscopy referral center. Gastrointest Endosc. 2012;76(2):255-263. doi:10.1016/j.gie.2012.02.060

14. Albéniz E, Pellisé $M$, Gimeno García AZ, et al. Clinical guidelines for endoscopic mucosal resection of non-pedunculated colorectal lesions. Gastroenterol Hepatol. 2018. doi:10.1016/j.gastrohep.2017.08.013

15. Albéniz E, Pellisé M, Gimeno-García AZ, et al. Clinical guidelines for endoscopic mucosal resection of non-pedunculated colorectal lesions. Rev Esp Enfermedades Dig. 2018. doi:10.17235/reed.2018.5086/2017

16. International Agency for Research on Cancer (IARC). GLOBOCAN 2018: Latest global cancer data. CA Cancer J Clin. 2018.

17. Atkin WS, Edwards R, Kralj-Hans I, et al. Once-only flexible sigmoidoscopy screening in prevention of colorectal cancer: a multicentre randomised controlled trial. Lancet. 2010. doi:10.1016/S0140-6736(10)60551-X

18. Winawer SJ, Zauber AG, Ho MN, et al. Prevention of colorectal cancer by colonoscopic polypectomy. The National Polyp Study Workgroup. N Engl J Med. 1993. doi:10.1056/NEJM199312303292701

19. Van Gossum A, Cozzoli A, Adler M, Taton G, Cremer M. Colonoscopic snare polypectomy: analysis of 1485 resections comparing two types of current. Gastrointest Endosc. 1992. doi:10.1016/S0016-5107(92)70479-9

20. Ferrara F, Luigiano C, Ghersi S, et al. Efficacy, safety and outcomes of 'inject and cut' endoscopic mucosal resection for large sessile and flat colorectal polyps. Digestion. 2010. doi:10.1159/000284397

21. Sanchez-Yague A, Kaltenbach $T$, Yamamoto $H$, Anglemyer $A$, Inoue $H$, Soetikno R. The endoscopic cap that can (with videos). Gastrointest Endosc. 2012. doi:10.1016/j.gie.2012.04.447 
22. Bourke M, Jideh B. How to Perform Wide-Field Endoscopic Mucosal Resection and Follow-up Examinations. Gastrointest Endosc Clin N Am. 2019. doi:10.1016/j.giec.2019.05.002

23. Tada $M$, Inoue $H$, Yabata $E$, Okabe $S$, Endo $M$. Feasibility of the transparent cap-fitted colonoscope for screening and mucosal resection. Dis Colon Rectum. 1997. doi:10.1007/BF02055390

24. Conio M, Blanchi S, Repici A, Ruggeri C, Fisher DA, Filiberti R. Cap-assisted endoscopic mucosal resection for colorectal polyps. Dis Colon Rectum. 2010. doi:10.1007/DCR.0b013e3181d95a54

25. Sanchez-Yague A, Kaltenbach T, Raju G, Soetikno R. Advanced endoscopic resection of colorectal lesions. Gastroenterol Clin North Am. 2013. doi:10.1016/j.gtc.2013.05.012

26. Wang AY, Flynn MM, Patrie JT, et al. Underwater endoscopic mucosal resection of colorectal neoplasia is easily learned, efficacious, and safe. Surg Endosc. 2014. doi:10.1007/s00464-013-3297-5

27. Kim HG, Thosani N, Banerjee S, Chen A, Friedland S. Underwater endoscopic mucosal resection for recurrences after previous piecemeal resection of colorectal polyps (with video). Gastrointest Endosc. 2014. doi:10.1016/j.gie.2014.05.318

28. Uedo N, Nemeth A, Johansson GW, Toth E, Thorlacius H. Underwater endoscopic mucosal resection of large colorectal lesions. Endoscopy. 2015. doi:10.1055/s-0034-1390749

29. Nett A, Binmoeller K. Underwater Endoscopic Mucosal Resection. Gastrointest Endosc Clin N Am. 2019. doi:10.1016/j.giec.2019.05.004

30. Masci E, Viale E, Notaristefano C, et al. Endoscopic mucosal resection in high- and low-volume centers: A prospective multicentric study. Surg Endosc. 2013. doi:10.1007/s00464-013-2977-5

31. Luigiano C, Consolo P, Scaffidi MG, et al. Endoscopic mucosal resection for large and giant sessile and flat colorectal polyps: A single-center experience with long-term follow-up. Endoscopy. 2009. doi:10.1055/s0029-1215091

32. Moss A, Williams SJ, Hourigan LF, et al. Long-term adenoma recurrence 


\section{Referencias}

following wide-field endoscopic mucosal resection (WF-EMR) for advanced colonic mucosal neoplasia is infrequent: Results and risk factors in 1000 cases from the Australian Colonic EMR (ACE) study. Gut. 2015. doi:10.1136/gutjnl-2013-305516

33. Hassan C, Pickhardt PJ, Di Giulio E, Hunink MGM, Zullo A, Nardelli BB. Value-of-information analysis to guide future research in the management of the colorectal malignant polyp. Dis Colon Rectum. 2010. doi:10.1007/DCR.0b013e3181c3be55

34. Heresbach D, Kornhauser R, Seyrig JA, et al. A national survey of endoscopic mucosal resection for superficial gastrointestinal neoplasia. Endoscopy. 2010. doi:10.1055/s-0030-1255715

35. Huang Y, Liu S, Gong W, Zhi F, Pan D, Jiang B. Clinicopathologic features and endoscopic mucosal resection of laterally spreading tumors: Experience from China. Int J Colorectal Dis. 2009. doi:10.1007/s00384-0090749-4

36. lishi $H$, Tatsuta $M$, Iseki $K$, et al. Endoscopic piecemeal resection with submucosal saline injection of large sessile colorectal polyps. Gastrointest Endosc. 2000. doi:10.1067/mge.2000.104652

37. Lim TR, Mahesh V, Singh S, et al. Endoscopic mucosal resection of colorectal polyps in typical UK hospitals. World J Gastroenterol. 2010. doi:10.3748/wjg.v16.i42.5324

38. Terasaki M, Tanaka S, Oka S, et al. Clinical outcomes of endoscopic submucosal dissection and endoscopic mucosal resection for laterally spreading tumors larger than 20mm. J Gastroenterol Hepatol. 2012. doi:10.1111/j.1440-1746.2011.06977.x

39. Lambert R, Tanaka S. Laterally spreading tumors in the colon and rectum. Eur J Gastroenterol Hepatol. 2012. doi:10.1097/MEG.0b013e328355e2d9

40. Uraoka T, Saito Y, Matsuda T, et al. Endoscopic indications for endoscopic mucosal resection of laterally spreading tumours in the colorectum. Gut. 2006. doi:10.1136/gut.2005.087452

41. Oka S, Tanaka S, Kanao H, Oba S, Chayama K. Therapeutic strategy for colorectal laterally spreading tumor. Dig Endosc. 2009. 
doi:10.1111/j.1443-1661.2009.00869.x

42. Albeniz E, Kaneko K, León-Brito H, et al. Sa1585 Morphological and Histological Characteristics of Colonic Laterally Spreading Tumors. East/WEST Comparative Study. Gastrointest Endosc. 2015. doi:10.1016/j.gie.2015.03.1374

43. Cotton PB, Eisen GM, Aabakken L, et al. A lexicon for endoscopic adverse events: report of an ASGE workshop. Gastrointest Endosc. 2010. doi:10.1016/j.gie.2009.10.027

44. Burgess NG, Metz AJ, Williams SJ, et al. Risk Factors for Intraprocedural and Clinically Significant Delayed Bleeding After Wide-field Endoscopic Mucosal Resection of Large Colonic Lesions. Clin Gastroenterol Hepatol. 2014. doi:10.1016/j.cgh.2013.09.049

45. Kim HS, Kim TI, Kim WH, et al. Risk factors for immediate postpolypectomy bleeding of the colon: A multicenter study. Am J Gastroenterol. 2006. doi:10.1111/j.1572-0241.2006.00638.x

46. Swan MP, Bourke MJ, Alexander S, Moss A, Williams SJ. Large refractory colonic polyps: is it time to change our practice? A prospective study of the clinical and economic impact of a tertiary referral colonic mucosal resection and polypectomy service (with videos). Gastrointest Endosc. 2009. doi:10.1016/j.gie.2009.05.039

47. Lüning $\mathrm{TH}$, Keemers-Gels ME, Barendregt WB, Tan ACITL, Rosman C. Colonoscopic perforations: A review of 30,366 patients. Surg Endosc Other Interv Tech. 2007. doi:10.1007/s00464-007-9251-7

48. Heldwein W, Dollhopf M, Rösch T, et al. The Munich Polypectomy Study (MUPS): Prospective analysis of complications and risk factors in 4000 colonic snare polypectomies. Endoscopy. 2005. doi:10.1055/s-2005870512

49. Taku K, Sano Y, Fu KI, et al. latrogenic perforation associated with therapeutic colonoscopy: A multicenter study in Japan. J Gastroenterol Hepatol. 2007. doi:10.1111/j.1440-1746.2007.05022.x

50. Burgess NG, Bassan MS, McLeod D, Williams SJ, Byth K, Bourke MJ. Deep mural injury and perforation after colonic endoscopic mucosal resection: $\mathrm{A}$ 


\section{Referencias}

new classification and analysis of risk factors. Gut. 2017. doi:10.1136/gutjnl-2015-309848

51. Bourke M. Endoscopic mucosal resection in the colon: A practical guide. Tech Gastrointest Endosc. 2011. doi:10.1016/j.tgie.2011.01.002

52. Bassan MS, Holt B, Moss A, Williams SJ, Sonson R, Bourke MJ. Carbon dioxide insufflation reduces number of postprocedure admissions after endoscopic resection of large colonic lesions: A prospective cohort study. Gastrointest Endosc. 2013. doi:10.1016/j.gie.2012.06.004

53. Nelson DB, McQuaid KR, Bond JH, Lieberman DA, Weiss DG, Johnston TK. Procedural success and complications of large-scale screening colonoscopy. Gastrointest Endosc. 2002. doi:10.1067/mge.2002.121883

54. Cha JM, Lim KS, Lee SH, et al. Clinical outcomes and risk factors of postpolypectomy coagulation syndrome: A multicenter, retrospective, casecontrol study. Endoscopy. 2013. doi:10.1055/s-0032-1326104

55. Lee SH, Kim KJ, Yang DH, et al. Postpolypectomy fever, a rare adverse event of polypectomy: Nested case-control study. Clin Endosc. 2014. doi:10.5946/ce.2014.47.3.236

56. Banerjee $\mathrm{S}$, Shen $\mathrm{B}$, Baron $\mathrm{TH}$, et al. Antibiotic prophylaxis for $\mathrm{Gl}$ endoscopy. Gastrointest Endosc. 2008. doi:10.1016/j.gie.2008.02.068

57. Vargo JJ, Cohen LB, Rex DK, Kwo PY. Position statement: nonanesthesiologist administration of propofol for $\mathrm{Gl}$ endoscopy. Gastrointest Endosc. 2009. doi:10.1016/j.gie.2009.07.020

58. Rex DK, Deenadayalu V, Eid E. Gastroenterologist-Directed Propofol: An Update. Gastrointest Endosc Clin N Am. 2008. doi:10.1016/j.giec.2008.06.002

59. Friedrich K, Stremmel W, Sieg A. Endoscopist-administered propofol sedation is safe - a prospective evaluation of 10,000 patients in an outpatient practice. J Gastrointest Liver Dis. 2012.

60. Sieg A, Beck S, Scholl SG, et al. Safety analysis of endoscopist-directed propofol sedation: A prospective, national multicenter study of 24441 patients in German outpatient practices. J Gastroenterol Hepatol. 2014. doi:10.1111/jgh.12458 
61. Ko CW, Dominitz JA. Complications of Colonoscopy: Magnitude and Management. Gastrointest Endosc Clin N Am. 2010. doi:10.1016/j.giec.2010.07.005

62. Radaelli F, Meucci G, Sgroi G, Minoli G. Technical performance of colonoscopy: The key role of sedation/analgesia and other quality indicators. Am J Gastroenterol. 2008. doi:10.1111/j.15720241.2007.01778.x

63. Hansen JJ, Ulmer BJ, Rex DK. Technical performance of colonoscopy in patients sedated with nurse-administered propofol. Am J Gastroenterol. 2004. doi:10.1046/j.1572-0241.2003.04022.x

64. McQuaid KR, Laine L. A systematic review and meta-analysis of randomized, controlled trials of moderate sedation for routine endoscopic procedures. Gastrointest Endosc. 2008. doi:10.1016/j.gie.2007.12.046

65. Sethi S, Wadhwa V, Thaker A, et al. Propofol versus traditional sedative agents for advanced endoscopic procedures: A meta-analysis. Dig Endosc. 2014. doi:10.1111/den.12219

66. Hurlstone DP, Sanders DS, Cross SS, George R, Shorthouse AJ, Brown S. A prospective analysis of extended endoscopic mucosal resection for large rectal villous adenomas: An alternative technique to transanal endoscopic microsurgery. Color Dis. 2005. doi:10.1111/j.1463-1318.2005.00813.x

67. Min BH, Chang DK, Kim DU, et al. Low frequency of bacteremia after an endoscopic resection for large colorectal tumors in spite of extensive submucosal exposure. Gastrointest Endosc. 2008. doi:10.1016/j.gie.2007.11.051

68. Piccolo G, Di Vita M, Cavallaro A, et al. Presentation and management of splenic injury after colonoscopy: A systematic review. Surg Laparosc Endosc Percutaneous Tech. 2014. doi:10.1097/SLE.0b013e3182a83493

69. Barendse RM, Oors JM, de Graaf EJR, et al. The effect of endoscopic mucosal resection and transanal endoscopic microsurgery on anorectal function. Color Dis. 2013. doi:10.1111/codi.12311

70. Ji JS, Lee SW, Kim TH, et al. Comparison of prophylactic clip and endoloop application for the prevention of postpolypectomy bleeding in 


\section{Referencias}

pedunculated colonic polyps: A prospective, randomized, multicenter study. Endoscopy. 2014. doi:10.1055/s-0034-1365515

71. Hassan C, Repici A, Sharma P, et al. Efficacy and safety of endoscopic resection of large colorectal polyps: A systematic review and metaanalysis. Gut. 2016. doi:10.1136/gutjnl-2014-308481

72. Mahadeva S, Rembacken BJ. Standard 'inject and cut' endoscopic mucosal resection technique is practical and effective in the management of superficial colorectal neoplasms. Surg Endosc. 2009. doi:10.1007/s00464008-9983-z

73. Watabe $H$, Yamaji $Y$, Okamoto $M$, et al. Risk assessment for delayed hemorrhagic complication of colonic polypectomy: polyp-related factors and patient-related factors\{A figure is presented\}. Gastrointest Endosc. 2006. doi:10.1016/j.gie.2006.02.054

74. R. H, Y. P, N. H, et al. Polysaccharide hemostatic system for hemostasis management in colorectal endoscopic mucosal resection. Dig Endosc. 2014. doi:10.1111/den.12054 LK http://novacat.nova.edu:4550/resserv?sid=EMBASE\&issn=09155635\&id=d oi:10.1111\%2Fden.12054\&atitle=Polysaccharide+hemostatic+system+for+ hemostasis+management+in+colorectal+endoscopic+mucosal+resection\& stitle=Dig.+Endosc.\&title=Digestive+Endoscopy\&volume=26\&issue=1\&spa ge $=63 \&$ epage $=68 \&$ aulast $=$ Huang \&aufirst $=$ Rui\&auinit $=$ R.\&aufull=Huang + R . \&coden=DIENE\&isbn=\&pages=63-68\&date=2014\&auinit1=R\&auinitm=

75. Bahin FF, Rasouli KN, Byth K, et al. Prediction of clinically significant bleeding following wide-field endoscopic resection of large sessile and laterally spreading colorectal lesions: A clinical risk score. Am J Gastroenterol. 2016. doi:10.1038/ajg.2016.235

76. Sawhney MS, Salfiti N, Nelson DB, Lederle FA, Bond JH. Risk factors for severe delayed postpolypectomy bleeding. Endoscopy. 2008. doi:10.1055/s-2007-966959

77. Lee CK, Lee SH, Park JY, et al. Prophylactic argon plasma coagulation ablation does not decrease delayed postpolypectomy bleeding. Gastrointest Endosc. 2009. doi:10.1016/j.gie.2008.11.024 
78. Metz AJ, Bourke MJ, Moss A, Williams SJ, Swan MP, Byth K. Factors that predict bleeding following endoscopic mucosal resection of large colonic lesions. Endoscopy. 2011. doi:10.1055/s-0030-1256346

79. Liaquat $H$, Rohn E, Rex DK. Prophylactic clip closure reduced the risk of delayed postpolypectomy hemorrhage: Experience in 277 clipped large sessile or flat colorectal lesions and 247 control lesions. Gastrointest Endosc. 2013. doi:10.1016/j.gie.2012.10.024

80. Qumseya BJ, Wolfsen C, Wang Y, et al. Factors associated with increased bleeding post-endoscopic mucosal resection. J Dig Dis. 2013. doi:10.1111/1751-2980.12002

81. Bahin FF, Naidoo M, Williams SJ, et al. Prophylactic endoscopic coagulation to prevent bleeding after wide-field endoscopic mucosal resection of large sessile colon polyps. Clin Gastroenterol Hepatol. 2015. doi:10.1016/j.cgh.2014.07.063

82. Katsinelos P, Gkagkalis S, Paroutoglou G, et al. A prospective comparative study of blended and pure coagulation current in endoscopic mucosal resection of large sessile colorectal polyps. Surg Laparosc Endosc Percutaneous Tech. 2014. doi:10.1097/SLE.0b013e31829ce99e

83. Elliott TR, Tsiamoulos ZP, Thomas-Gibson S, et al. Factors associated with delayed bleeding after resection of large nonpedunculated colorectal polyps. Endoscopy. 2018. doi:10.1055/a-0577-3206

84. Kim GU, Seo M, Song EM, et al. Association between the ulcer status and the risk of delayed bleeding after the endoscopic mucosal resection of colon. J Gastroenterol Hepatol. 2017. doi:10.1111/jgh.13804

85. Albeniz E, Fraile M, MartíNez-Ares D, et al. 332 Delayed Bleeding Risk Score for Colorectal Endoscopic Mucosal Resection. Gastrointest Endosc. 2015. doi:10.1016/j.gie.2015.03.1209

86. Dior $\mathrm{M}$, Coriat $\mathrm{R}$, Tarabichi S, et al. Does endoscopic mucosal resection for large colorectal polyps allow ambulatory management? Surg Endosc. 2013. doi:10.1007/s00464-013-2807-9

87. Dobrowolski S, Dobosz M, Babicki A, Dymecki D, Hać S. Prophylactic submucosal saline-adrenaline injection in colonoscopic polypectomy: 


\section{Referencias}

Prospective randomized study. Surg Endosc Other Interv Tech. 2004. doi:10.1007/s00464-003-9214-6

88. Hsieh $\mathrm{YH}$, Lin $\mathrm{HJ}$, Tseng GY, et al. Is submucosal epinephrine injection necessary before polypectomy? A prospective, comparative study. Hepatogastroenterology. 2001.

89. Lee SH, Chung IK, Kim SJ, et al. Comparison of postpolypectomy bleeding between epinephrine and saline submucosal injection for large colon polyps by conventional polypectomy: A prospective randomized, multicenter study. World J Gastroenterol. 2007. doi:10.3748/wjg.v13.i21.2973

90. Katsinelos P, Kountouras J, Paroutoglou G, et al. A comparative study of $50 \%$ dextrose and normal saline solution on their ability to create submucosal fluid cushions for endoscopic resection of sessile rectosigmoid polyps. Gastrointest Endosc. 2008. doi:10.1016/j.gie.2008.02.063

91. Yoshida $N$, Naito $Y$, Inada $Y$, et al. Endoscopic mucosal resection with $0.13 \%$ hyaluronic acid solution for colorectal polyps less than $20 \mathrm{~mm}$ : A randomized controlled trial. J Gastroenterol Hepatol. 2012. doi:10.1111/j.1440-1746.2012.07166.x

92. Kishihara $T$, Chino A, Uragami N, et al. Usefulness of sodium hyaluronate solution in colorectal endoscopic mucosal resection. Dig Endosc. 2012. doi:10.1111/j.1443-1661.2012.01244.x

93. Uraoka T, Fujii T, Saito $Y$, et al. Effectiveness of glycerol as a submucosal injection for EMR. Gastrointest Endosc. 2005. doi:10.1016/S00165107(05)00321-4

94. Lee HS, Jeon SW, Kwon YH, et al. Prophylactic endoscopic coagulation to prevent delayed post-EMR bleeding in the colorectum: a prospective randomized controlled trial (with videos). Gastrointest Endosc. 2019. doi:10.1016/j.gie.2019.05.039

95. Fahrtash-Bahin F, Holt BA, Jayasekeran V, Williams SJ, Sonson R, Bourke MJ. Snare tip soft coagulation achieves effective and safe endoscopic hemostasis during wide-field endoscopic resection of large colonic lesions (with videos). Gastrointest Endosc. 2013. doi:10.1016/j.gie.2013.02.030 
96. Parra-Blanco A, Kaminaga N, Kojima T, et al. Hemoclipping for postpolypectomy and postbiopsy colonic bleeding. Gastrointest Endosc. 2000. doi:10.1016/S0016-5107(00)70384-1

97. Sobrino-Faya M, Martínez S, Gómez Balado $M$, et al. Clips for the prevention and treatment of postpolypectomy bleeding (hemoclips in polypectomy). Rev Esp Enfermedades Dig. 2002.

98. Shioji K, Suzuki Y, Kobayashi M, et al. Prophylactic clip application does not decrease delayed bleeding after colonoscopic polypectomy. Gastrointest Endosc. 2003. doi:10.1067/mge.2003.193

99. Dokoshi T, Fujiya $M$, Tanaka $K$, et al. A randomized study on the effectiveness of prophylactic clipping during endoscopic resection of colon polyps for the prevention of delayed bleeding. Biomed Res Int. 2015. doi:10.1155/2015/490272

100. Mori $\mathrm{H}$, Kobara $\mathrm{H}$, Nishiyama $\mathrm{N}$, et al. Simple and reliable treatment for post-EMR artificial ulcer floor with snare cauterization for $10-$ to $20-\mathrm{mm}$ colorectal polyps: a randomized prospective study (with video). Surg Endosc. 2015. doi:10.1007/s00464-014-3983-y

101. Matsumoto $\mathrm{M}$, Kato $\mathrm{M}$, Oba K, et al. Multicenter randomized controlled study to assess the effect of prophylactic clipping on post-polypectomy delayed bleeding. Dig Endosc. 2016. doi:10.1111/den.12661

102. Zhang QS, Han B, Xu JH, Gao P, Shen YC. Clip closure of defect after endoscopic resection in patients with larger colorectal tumors decreased the adverse events. Gastrointest Endosc. 2015. doi:10.1016/j.gie.2015.04.005

103. Nishizawa T, Suzuki H, Goto O, Ogata H, Kanai T, Yahagi N. Effect of prophylactic clipping in colorectal endoscopic resection: A meta-analysis of randomized controlled studies. United Eur Gastroenterol J. 2017. doi:10.1177/2050640616687837

104. Ayoub F, Westerveld DR, Forde JJ, Forsmark CE, Draganov P V., Yang D. Effect of prophylactic clip placement following endoscopic mucosal resection of large colorectal lesions on delayed polypectomy bleeding: A meta-analysis. World J Gastroenterol. 2019. doi:10.3748/wjg.v25.i18.2251 


\section{Referencias}

105. Burgess NG, Williams SJ, Hourigan LF, et al. A management algorithm based on delayed bleeding after wide-field endoscopic mucosal resection of large colonic lesions. Clin Gastroenterol Hepatol. 2014. doi:10.1016/j.cgh.2014.01.026

106. van der Star S, Moons LMG, ter Borg F, et al. Management of delayed bleeding after endoscopic mucosal resection of large colorectal polyps: a retrospective multi-center cohort study. Endosc Int Open. 2020. doi:10.1055/a-1192-3816

107. Nishiyama N, Mori $\mathrm{H}$, Kobara H, et al. Efficacy and safety of over-the-scope clip: Including complications after endoscopic submucosal dissection. World J Gastroenterol. 2013. doi:10.3748/wjg.v19.i18.2752

108. Chahal D, Lee JGH, Ali-Mohamad N, Donnellan F. High rate of re-bleeding after application of Hemospray for upper and lower gastrointestinal bleeds. Dig Liver Dis. 2020. doi:10.1016/j.dld.2020.01.009

109. Soons E, Turan A, van Geenen E, Siersema P. Application of a novel selfassembling peptide to prevent hemorrhage after EMR, a feasibility and safety study. Surg Endosc. 2020. doi:10.1007/s00464-020-07819-7

110. Bon I, Bartoli R, Lorenzo-Zúniga V. Endoscopic shielding technique, a new method in therapeutic endoscopy. World J Gastroenterol. 2017. doi:10.3748/wjg.v23.i21.3761

111. Martines G, Picciariello A, Dibra R, et al. Efficacy of cyanoacrylate in the prevention of delayed bleeding after endoscopic mucosal resection of large colorectal polyps: a pilot study. Int J Colorectal Dis. 2020. doi:10.1007/s00384-020-03678-9

112. Albéniz E, Fraile M, Ibáñez B, et al. A Scoring System to Determine Risk of Delayed Bleeding After Endoscopic Mucosal Resection of Large Colorectal Lesions. Clin Gastroenterol Hepatol. 2016. doi:10.1016/j.cgh.2016.03.021

113. Bahin FF, Rasouli KN, Byth K, et al. Prediction of clinically significant bleeding following wide-field endoscopic resection of large sessile and laterally spreading colorectal lesions: A clinical risk score. Am J Gastroenterol. 2016. doi:10.1038/ajg.2016.235

114. Moons KGM, Kengne AP, Grobbee DE, et al. Risk prediction models: II. 
External validation, model updating, and impact assessment. Heart. 2012. doi:10.1136/heartjnl-2011-301247

115. Albéniz E, Gimeno-García $A Z$, Fraile $M$, et al. Clinical validation of risk scoring systems to predict risk of delayed bleeding after EMR of large colorectal lesions. Gastrointest Endosc. 2020. doi:10.1016/j.gie.2019.10.013

116. Albeniz E, Alvarez-Gonzalez M, Espinós J, et al. CLIP CLOSURE OF LARGE NON-PEDUNCULATED COLON POLYPS WITH AVERAGE AND HIGH RISK OF DELAYED BLEEDING. In: ESGE Days 2019. ; 2019. doi:10.1055/s-00391681264

117. Albéniz E, Álvarez MA, Espinós JC, et al. Clip Closure After Resection of Large Colorectal Lesions With Substantial Risk of Bleeding. Gastroenterology. 2019;157(5):1213-1221.e4. doi:10.1053/j.gastro.2019.07.037

118. Feagins LA, Smith AD, Kim D, et al. Efficacy of Prophylactic Hemoclips in Prevention of Delayed Post-Polypectomy Bleeding in Patients With Large Colonic Polyps. Gastroenterology. 2019;157(4):967-976.e1. doi:10.1053/j.gastro.2019.05.003

119. Pohl H, Grimm IS, Moyer MT, et al. Clip Closure Prevents Bleeding After Endoscopic Resection of Large Colon Polyps in a Randomized Trial. Gastroenterology. 2019. doi:10.1053/j.gastro.2019.03.019

120. Albéniz E, Álvarez MA, Espinós JC, et al. Clip Closure After Resection of Large Colorectal Lesions With Substantial Risk of Bleeding. Gastroenterology. 2019. doi:10.1053/j.gastro.2019.07.037

121. Spadaccini $M$, Albéniz $E$, Pohl $H$, et al. Prophylactic Clipping After Colorectal Endoscopic Resection Prevents Bleeding of Large, Proximal Polyps: Meta-analysis of Randomized Trials. Gastroenterology. 2020. doi:10.1053/j.gastro.2020.03.051

122. Parikh ND, Zanocco K, Keswani RN, Gawron AJ. A cost-efficacy decision analysis of prophylactic clip placement after endoscopic removal of large polyps. Clin Gastroenterol Hepatol. 2013. doi:10.1016/j.cgh.2012.12.044

123. Bahin FF, Rasouli KN, Williams SJ, Lee EYT, Bourke MJ. Prophylactic 


\section{Referencias}

clipping for the prevention of bleeding following wide-field endoscopic mucosal resection of laterally spreading colorectal lesions: An economic modeling study. Endoscopy. 2016. doi:10.1055/s-0042-105558

124. Shah ED, Pohl H, Rex DK, Morales SJ, Feagins LA, Law R. Routine Prophylactic Clip Closure is Cost Saving After Endoscopic Resection of Large Colon Polyps in a Medicare Population: Budget impact analysis: clip closure after resecting large colon polyps. Gastroenterology. November 2019. doi:10.1053/j.gastro.2019.11.015

125. Albéniz E, Gimeno-García $A Z$, Fraile $M$, et al. Clinical validation of risk scoring systems to predict risk of delayed bleeding after EMR of large colorectal lesions. Gastrointest Endosc. October 2019. doi:10.1016/j.gie.2019.10.013

126. Albéniz E, Álvarez MA, Espinós JC, et al. Clip Closure After Resection of Large Colorectal Lesions With Substantial Risk of Bleeding. Gastroenterology. 2019. doi:10.1053/j.gastro.2019.07.037

127. RESOLUCIÓN 1564/2018, de 20 de Diciembre, Del Director Gerente Del Servicio Navarro de Salud-Osasunbidea, Por La Que Se Establecen Las Tarifas Por Los Servicios Prestados Por El Servicio Navarro de SaludOsasunbidea., - Boletín Oficial de Navarra, de 22-0. Spain; 2019.

128. Ibarrondo O, Álvarez-López I, Freundlich F, et al. Probabilistic cost-utility analysis and expected value of perfect information for the Oncotype multigenic test: a discrete event simulation model. Gac Sanit. 2018. doi:10.1016/j.gaceta.2018.07.012

129. Doble B, Pufulete M, Harris JM, et al. Health-related quality of life impact of minor and major bleeding events during dual antiplatelet therapy: A systematic literature review and patient preference elicitation study. Health Qual Life Outcomes. 2018. doi:10.1186/s12955-018-1019-3

130. Hutubessy RCW, Baltussen R, Tan Torres-Edejer T, Evans DB. WHOCHOICE: Choosing interventions that are cost-effective. Heal Syst Perform Assess debates, methods empiricism Geneva WHO Ed. 2003.

131. Bass AJ, Thorsson V, Shmulevich I, et al. Comprehensive molecular characterization of gastric adenocarcinoma. Nature. 2014. 
doi:10.1038/nature13480

132. Buddingh KT, Herngreen T, Haringsma J, et al. Location in the right hemicolon is an independent risk factor for delayed post-polypectomy hemorrhage: A multi-center case-control study. Am J Gastroenterol. 2011. doi:10.1038/ajg.2010.507

133. E. A, M. F, D. M-A, et al. Delayed bleeding after endoscopic mucosal resection of large colorectal lesions. A prospective multicenter spanish study. Gastrointest Endosc. 2014. doi:10.1016/j.gie.2014.02.1041 LK http://ucelinks.cdlib.org:8888/sfx_ucsf?sid=EMBASE\&issn=00165107\&id= doi:10.1016\%2Fj.gie.2014.02.1041\&atitle=Delayed+bleeding+after+endos copic+mucosal+resection+of+large+colorectal+lesions.+A+prospective+mu Iticenter+spanish+study\&stitle=Gastrointest.+Endosc.\&title=Gastrointesti nal+Endoscopy $\&$ volume $=79 \&$ issue $=5 \&$ spage $=A B 438 \&$ epage $=A B 439 \&$ aulas $\mathrm{t}=$ Albeniz\&aufirst=Eduardo\&auinit=E.\&aufull=Albeniz $+E . \& \operatorname{coden}=\& i s b n=\&$ pages $=A B 438-A B 439 \&$ date $=2014 \&$ auinit1 $=E \&$ auinitm $=$

134. Shiffman ML, Farrel MT, Yee YS. Risk of bleeding after endoscopic biopsy or polypectomy in patients taking aspirin or other NSAIDs. Gastrointest Endosc. 1994. doi:10.1016/S0016-5107(94)70210-1

135. Manocha D, Singh M, Mehta N, Murthy UK. Bleeding risk after invasive procedures in aspirin/NSAID Users: Polypectomy study in veterans. Am J Med. 2012. doi:10.1016/j.amjmed.2012.05.030

136. Pan A, Schlup M, Lubcke R, Chou A, Schultz M. The role of aspirin in postpolypectomy bleeding - a retrospective survey. BMC Gastroenterol. 2012. doi:10.1186/1471-230X-12-138

137. Hui AJ, Wong RMY, Ching JYL, Hung LCT, Chung SCS, Sung JJY. Risk of colonoscopic polypectomy bleeding with anticoagulants and antiplatelet agents: Analysis of 1657 cases. Gastrointest Endosc. 2004. doi:10.1016/S0016-5107(03)02307-1

138. Sorbi D, Norton I, Conio M, Balm R, Zinsmeister A, Gostout CJ. Postpolypectomy lower GI bleeding: Descriptive analysis. Gastrointest Endosc. 2000. doi:10.1067/mge.2000.105773

139. Buchner AM, Guarner-Argente C, Ginsberg GG. Outcomes of EMR of 
Referencias

defiant colorectal lesions directed to an endoscopy referral center. Gastrointest Endosc. 2012. doi:10.1016/j.gie.2012.02.060

140. Park CH, Jung YS, Nam E, Eun CS, Park D II, Han DS. Comparison of Efficacy of Prophylactic Endoscopic Therapies for Postpolypectomy Bleeding in the Colorectum: A Systematic Review and Network Meta-Analysis. Am J Gastroenterol. 2016. doi:10.1038/ajg.2016.287

141. Boumitri C, Mir FA, Ashraf I, et al. Prophylactic clipping and postpolypectomy bleeding: A metaanalysis and systematic review. Ann Gastroenterol. 2016. doi:10.20524/aog.2016.0075

142. Loras C, Mayor V, Fernández-Bañares F, Esteve M. Study of the standard direct costs of various techniques of advanced endoscopy. Comparison with surgical alternatives. Dig Liver Dis. 2018;50(7):689-697. doi:10.1016/j.dld.2018.03.002

143. Nomura T, Kobayashi M, Morikawa T, Horiki N. Clip-fixed endoloop: An efficacious new method for mucosal defect closure. Endoscopy. 2018. doi:10.1055/s-0044-101025 


\section{APÉNDICE}

\subsection{PUBLICACIONES}

Publicación preliminar 1: Albéniz $E$, Fraile $M$, Ibáñez $B$, Alonso-Aguirre $P$, Martínez-Ares D, Soto S, Gargallo CJ, Ramos Zabala F, Álvarez MA, RodríguezSánchez J, Múgica F, Nogales Ó, Herreros de Tejada A, Redondo E, Pin N, LeónBrito $H$, Pardeiro $R$, López-Roses $L$, Rodríguez-Téllez $M$, Jiménez $A$, MartínezAlcalá F, García O, de la Peña J, Ono A, Alberca de Las Parras F, Pellisé M, Rivero L, Saperas E, Pérez-Roldán F, Pueyo Royo A, Eguaras Ros J, Zúñiga Ripa A, Concepción-Martín M, Huelin-Álvarez P, Colán-Hernández J, Cubiella J, Remedios D, Bessa I Caserras X, López-Viedma B, Cobian J, González-Haba M, Santiago J, Martínez-Cara JG, Valdivielso E, Guarner-Argente C; Endoscopic Mucosal Resection Endoscopic Spanish Society Group. A Scoring System to Determine Risk of Delayed Bleeding After Endoscopic Mucosal Resection of Large Colorectal Lesions. Clin Gastroenterol Hepatol. 2016 Aug; 14 (8):1140-7. doi: 10.1016/j.cgh.2016.03.021. Epub 2016 Mar 24.

Publicación 2: Albéniz E, Gimeno-García AZ, Fraile M, Ibáñez B, Guarner-Argente C, Alonso-Aguirre $P$, Álvarez MA, Gargallo CJ, Pellisé $M$, Ramos Zabala F, Herreros de Tejada A, Nogales Ó, Martínez-Ares D, Múgica F, de la Peña J, Espinós J, Huerta A, Álvarez A, Gonzalez-Santiago JM, Navajas F, Martínez-Cara JG, Redondo-Cerezo E, Merlo Mas J, Sábado F, Rivero L, Saperas E, Soto S, RodríguezSánchez J, López-Roses L, Rodríguez-Téllez M, Rullán Iriarte $M$, Elosua González A, Pardeiro R, Valdivielso Cortázar E, Concepción-Martín M, Huelin Álvarez $P$, Colán Hernández J, Cobian J, Santiago J, Jiménez A, Remedios D, López-Viedma B, García O, Martínez-Alcalá F, Pérez-Roldán F, Carbó J, Enguita M. Clinical validation of risk scoring systems to predict risk of delayed bleeding after EMR of large colorectal lesions. Gastrointest Endosc. 2020 Apr; 91 (4):868-878.e3. doi: 10.1016/j.gie.2019.10.013. Epub 2019 Oct 23. PMID: 31655045.

Publicación 3: Albéniz E, Álvarez MA, Espinós JC, Nogales O, Guarner C, Alonso P, Rodríguez-Téllez $M$, Herreros de Tejada $A$, Santiago J, Bustamante-Balén $M$, Rodríguez Sánchez J, Ramos-Zabala $F$, Valdivielso $E$, Martínez-Alcalá $F$, Fraile $M$, Elosua A, Guerra Veloz MF, Ibáñez Beroiz B, Capdevila F, Enguita-Germán M. Clip Closure After Resection of Large Colorectal Lesions With Substantial Risk of Bleeding. Gastroenterology. 2019 Nov; 157 (5):1213-1221.e4. doi: 10.1053/j.gastro.2019.07.037. Epub 2019 Jul 27. PMID: 31362007. 
Publicación 4: Spadaccini M, Albéniz E (co-first author), Pohl H, Maselli R, Thoguluva Chandrasekar V, Correale L, Anderloni A, Carrara S, Fugazza A, Badalamenti M, Iwatate M, Antonelli G, Enguita-Germán M, Álvarez MA, Sharma P, Rex DK, Hassan C, Repici A. Prophylactic Clipping After Colorectal Endoscopic Resection Prevents Bleeding of Large, Proximal Polyps: Meta-analysis of Randomized Trials. Gastroenterology. 2020 Jul; 159 (1):148-158.e11. doi: 10.1053/j.gastro.2020.03.051. Epub 2020 Apr 1. PMID: 32247023.

Publicación 5: Albéniz E, Enguita-Germán M (co-first author), Gimeno-García AZ, Herreros de Tejada A, Nogales O, Espinós JC, Rodríguez Sánchez J, Rosón P, Guarner C, Marín JC, Bhandari P, Spadaccini M, Repici A, Hassan C, ÁlvarezGonzález MA, Ibáñez Beroiz B. The Answer to "When to Clip" After Colorectal Endoscopic Mucosal Resection Based on a Cost-Effectiveness Analysis. Am J Gastroenterol. 2020 Nov 3. doi: 10.14309/ajg.0000000000000943. Epub ahead of print. PMID: 33149001. 


\section{A Scoring System to Determine Risk of Delayed Bleeding After Endoscopic Mucosal Resection of Large Colorectal Lesions}

Eduardo Albéniz,, María Fraile, ${ }^{*}$ Berta lbáñez,, Pedro Alonso-Aguirre, ${ }^{\S}$ David Martínez-Ares, Santiago Soto, " Carla Jerusalén Gargallo, ${ }^{\#}$ Felipe Ramos Zabala, ${ }^{* *}$ Marco Antonio Álvarez,, Joaquín Rodríguez-Sánchez, ${ }^{\S \S}$ Fernando Múgica, "l| Óscar Nogales, " Alberto Herreros de Tejada, ${ }^{\# \#}$ Eduardo Redondo, ${ }^{* \star \star}$ Carlos Guarner-Argente, ${ }^{\neq \neq \ddagger}$ Noel Pin, ${ }^{\S}$ Helena León-Brito, ${ }^{*}$ Remedios Pardeiro, ${ }^{\S}$ Leopoldo López-Roses, ${ }^{\S \S}$ Manuel Rodríguez-Téllez, \|\|\|\| Alejandra Jiménez, \|\|$\| \mid$ Felipe Martínez-Alcalá,

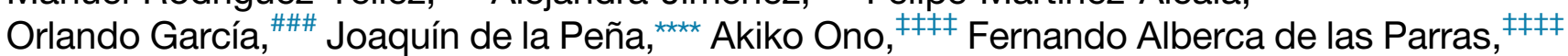
María Pellisé, ${ }^{\S \S \S \S}$ Liseth Rivero, ${ }^{\S \S \S \S}$ Esteban Saperas, $\||||| \mid$ Francisco Pérez-Roldán, Antonio Pueyo Royo, ${ }^{*}$ Javier Eguaras Ros, ${ }^{*}$ Alba Zúñiga Ripa, ${ }^{*}$ Mar Concepción-Martín, ${ }^{\ddagger \neq \neq}$ Patricia Huelin-Álvarez, ${ }^{\neq \neq \ddagger}$ Juan Colán-Hernández, ${ }^{\neq \neq \neq}$Joaquín Cubiella, ${ }^{\natural}$ David Remedios, Xavier Bessa i Caserras, ${ }^{\neq \ddagger}$ Bartolomé López-Viedma, ${ }^{\S \S}$ Julyssa Cobian, Mariano González-Haba, ${ }^{\# \# ~ J o s e ́ ~ S a n t i a g o, ~}{ }^{\# \# ~ J u a n ~ G a b r i e l ~ M a r t i ́ n e z-C a r a, ~}{ }^{\star \star \star}$ and Eduardo Valdivielso, ${ }^{\S}$ on behalf of the Endoscopic Mucosal Resection Endoscopic Spanish Society Group

${ }^{*}$ Complejo Hospitalario de Navarra, Pamplona, Spain; ${ }^{\ddagger}$ NavarraBiomed-Fundación Miguel Servet and Red de Investigación en Servicios de Salud en Enfermedades Crónicas, Pamplona, Spain; ${ }^{\S}$ Hospital Juan Canalejo, La Coruña, Spain; "Complejo Hospitalario de Vigo, Vigo, Spain; " Complejo Hospitalario de Ourense, Ourense, Spain; \#Hospital Clínico Universitario Lozano Blesa, Zaragoza, Spain; **Hospital Universitario HM Montepríncipe, Madrid, Spain; ${ }^{\ddagger \neq}$ Hospital del Mar, Barcelona, Spain; $\$$ Hospital General de Ciudad Real, Ciudad Real, Spain; "llHospital Universitario Donostia, Donostia-San Sebastián, Spain; "1\% Hospital General Universitario Gregorio Marañón, Madrid, Spain; \#\#Hospital Universitario Puerta de Hierro, Madrid, Spain; ***Hospital Universitario Virgen de las Nieves, Granada, Spain; ${ }^{\sharp \neq}$ Hospital de la Santa Creu y Sant Pau, Universitat Autònoma

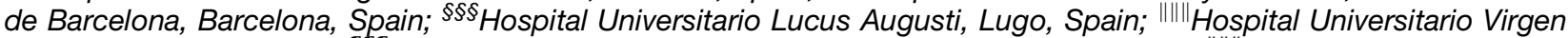
Macarena, Sevilla, Spain; " 17 Centro Andaluz de Gastroenterología Integral, Sevilla, Spain; \#\#\#ospital Moisès Broggi, Sant Joan Despí, Spain; ${ }^{* * *}$ Hospital de Valdecilla, Santander, Spain; ${ }^{+\neq \neq}$Hospital Universitario Virgen de la Arrixaca, Murcia, Spain;

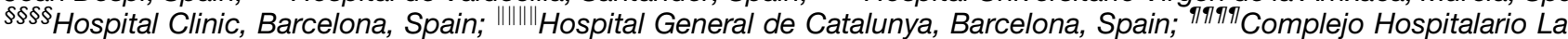
Mancha Centro, Alcázar de San Juan, Ciudad Real, Spain

BACKGROUND \& AIMS:

After endoscopic mucosal resection (EMR) of colorectal lesions, delayed bleeding is the most common serious complication, but there are no guidelines for its prevention. We aimed to identify risk factors associated with delayed bleeding that required medical attention after discharge until day 15 and develop a scoring system to identify patients at risk.

METHODS:

We performed a prospective study of 1214 consecutive patients with nonpedunculated colorectal lesions $20 \mathrm{~mm}$ or larger treated by EMR $(n=1255)$ at 23 hospitals in Spain, from February 2013 through February 2015. Patients were examined 15 days after the procedure, and medical data were collected. We used the data to create a delayed bleeding scoring system, and assigned a weight to each risk factor based on the $\beta$ parameter from multivariate logistic regression analysis. Patients were classified as being at low, average, or high risk for delayed bleeding.

RESULTS:

Delayed bleeding occurred in 46 cases (3.7\%, 95\% confidence interval, $2.7 \%-4.9 \%$ ). In multivariate analysis, factors associated with delayed bleeding included age $\geq 75$ years (odds ratio [OR], 2.36; $P<.01$ ), American Society of Anesthesiologist classification scores of III

Abbreviations used in this paper: ASA, American Society of Anesthesiologists; $\mathrm{Cl}$, confidence interval; DB, delayed bleeding; EMR, endoscopic mucosal resection; OR, odds ratio; $\mathrm{ROC}$, receiver operating characteristic.

\author{
(C) 2016 by the AGA Institute \\ $1542-3565 / \$ 36.00$ \\ http://dx.doi.org/10.1016/j.cgh.2016.03.021
}


or IV (OR, 1.90; $P \leq .05)$, aspirin use during EMR (OR, 3.16; $P<.05)$, right-sided lesions $(0 R, 4.86 ; P<.01)$, lesion size $\geq 40 \mathrm{~mm}(0 R, 1.91 ; P \leq .05)$, and a mucosal gap not closed by hemoclips (OR, 3.63; $P \leq .01)$. We developed a risk scoring system based on these 6 variables that assigned patients to the low-risk (score, 0-3), average-risk (score, 4-7), or high-risk (score, 8-10) categories with a receiver operating characteristic curve of 0.77 (95\% confidence interval, $0.70-0.83$ ). In these groups, the probabilities of delayed bleeding were $0.6 \%, 5.5 \%$, and $40 \%$, respectively.

CONCLUSIONS:

The risk of delayed bleeding after EMR of large colorectal lesions is 3.7\%. We developed a risk scoring system based on 6 factors that determined the risk for delayed bleeding (receiver operating characteristic curve, 0.77). The factors most strongly associated with delayed bleeding were right-sided lesions, aspirin use, and mucosal defects not closed by hemoclips. Patients considered to be high risk (score, 8-10) had a $40 \%$ probability of delayed bleeding.

Keywords: ASA; Mucosectomy; Colon Cancer; Prognostic Factor.

See similar article on page 1148.

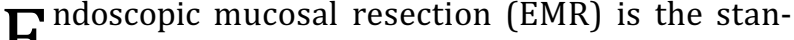

E dard treatment for superficial colorectal lesions in Western countries. ${ }^{1}$ Delayed bleeding (DB) has an incidence of $2.6 \%-9.7 \%$ after EMR of large colorectal lesions $(\geq 2 \mathrm{~cm}){ }^{2,3}$ Several features have been established as risk factors for DB, such as large lesions and location in the proximal colon, but others remain controversial. ${ }^{2,4-8}$

To prevent DB, several strategies commonly are used, such as adding adrenaline to the submucosal injection solution, ${ }^{9-11}$ applying argon plasma coagulation or coagulation forceps on nonbleeding visible vessels, ${ }^{12,13}$ and clipping to close the resection mucosal defect. Scientific evidence for all of these prevention strategies is scarce, and guidelines for prevention are lacking. ${ }^{14-21}$

In this study we aimed to identify risk factors and prevention strategies associated with DB after EMR of large nonpedunculated colorectal lesions, and to develop a simple DB risk score.

\section{Patients and Methods}

\section{Patients}

The Spanish EMR Group, formed by endoscopists from 23 Spanish hospitals, started a multicenter prospective observational study in February 2013. Nonpedunculated colorectal lesions $2 \mathrm{~cm}$ or larger that underwent EMR were recorded consecutively in a centralized database. All patients who had indications for EMR of colorectal lesions were screened for inclusion. Subjects who refused to participate in the study and/or had lesions smaller than $20 \mathrm{~mm}$ were excluded. Until February 2015, we collected patient characteristics, lesion features, and EMR technique using a standardized data record sheet.

All participants provided written informed consent for EMR and for inclusion in this study. The Ethics Committee at each participating center approved the study protocol.

\section{Endoscopic Mucosal Resection Technique and Special Conditions}

Experienced senior endoscopists performed the procedures using conventional or high-definition white-light endoscopes. Injection-assisted EMR was performed according to the description in the literature. ${ }^{22}$ Lesion size was measured either with open forceps or with the longitudinal size of an open snare. The mucosal defect was closed in some cases at the discretion of the endoscopist, using simple clipping (clips approaching the mucosal edges) or the "loop-clip" method (first, a polyloop is attached with clips around the mucosal edges, then the loop is closed to approach these edges, and, finally, additional clips are used to achieved complete closure if necessary). ${ }^{23}$

The electrocautery current used was not standardized and it depended on the endoscopist's preferences and institution availability. Because of the variability observed, we recorded the type of electrocautery current used (coagulation, cut, or endocut), and the electrocautery model, but not the cautery settings.

Before the procedure, an international normalized ratio of less than 1.5 was confirmed in patients taking anticoagulation therapy, and antiplatelet agents were ceased or not and restarted depending on comorbidities and each institution's clinical guidelines.

All participants were contacted by telephone or had a personal interview as of 15 days after the EMR. Follow-up evaluation was performed by outpatient services at each center and included follow-up endoscopic examinations.

\section{Outcomes and Definitions}

The main outcomes were to determine risk factors associated with DB after EMR of large nonpedunculated colorectal lesions, and to develop a simple predictive DB risk score.

We defined DB after EMR as clinically evident bleeding that required medical attention after discharge from the endoscopy unit until day 15 after the procedure. ${ }^{24}$ 


\section{Statistical Analysis}

Characteristics of the sample were described using descriptive statistics. Statistical significance was considered at a $P$ value less than .05. The risk of DB was estimated using sample proportions with 95\% confidence intervals (95\% CIs). Univariate logistic regression models were fitted to identify risk factors associated with DB. We entered variables that were significant at an $\alpha$ value of .1 into a multivariate regression model and used these as final predictive factors if the $P$ value was less than .1. Odds ratios (ORs) and 95\% CIs were calculated using multivariate analysis. Discrimination of the model was determined by calculating the area under the receiver operating characteristic (ROC) curve with its $95 \%$ CI. We assessed the calibration of the model with the Hosmer-Lemeshow goodness-of-fit-test, complemented with calibration plots using predicted risk against the actual risk in each decile. We developed a score by assigning a weight to each risk factor category based on the $\beta$ parameter from the multivariate logistic regression. From the continuous score, we classified patients into 3 risk categories: low, average, and high. The model was validated internally using the resampling validation method for logistic models with 150 bootstrap re-samples. Statistical analysis was performed using IBM Statistical Package for the Social Sciences (SPSS) program version 20.0 (SPSS, Inc, Chicago, IL) and R 3.1.0.

\section{Results}

\section{General Characteristics of Patients and Lesions}

A total of 1255 EMRs of large colorectal lesions were performed consecutively in 1214 patients (Table 1). There were $770(63.4 \%)$ males and their mean age was 67.9 years (range, 24-93 y). The American Society of Anesthesiologists (ASA) score was III or IV in 379 patients (30.5\%). A total of $218(17.5 \%)$ patients had antiplatelet therapy prescribed. Aspirin treatment was used during the procedure in 51 patients $(4.1 \%)$.

The mean lesion size was $30.5 \mathrm{~mm}$ (SD, $11.82 \mathrm{~mm}$; range $20-120 \mathrm{~mm}$ ). Lesion location was proximal to the splenic flexure in 51.3\%. Histology showed deep submucosal involvement in 81 patients $(6.8 \%)$.

\section{Endoscopic Mucosal Resection Characteristics}

The most commonly used solution for submucosal injection was saline in 827 patients $(66.2 \%)$, followed by glycerol in 150 patients (12\%). In 841 (67.4\%) EMRs, a $1: 10,000$ adrenaline dilution was added to these fluids. After the EMR, 281 (22.6\%) defects were closed completely (mean size, $27.4 \mathrm{~mm}$; SD, $8.8 \mathrm{~mm}$; range, 20-70 mm), $43.7 \%$ were located in the cecum or ascending colon. The median number of clips placed per mucosal defect was 3 (range, 2-10). Argon plasma was applied exclusively as a coagulation method in $84(6.7 \%)$ lesions. The intraoperative bleeding rate was 5.3\% (95\% CI, 4.14-6.72).

\section{Delayed Bleeding Rate and Predictors}

DB occurred in 46 patients $(3.7 \%$; $95 \% \mathrm{CI}, 2.7 \%$ 4.9\%): within 48 hours in 17 patients $(37 \%)$ and between 3 and 7 days in 23 patients (50\%). The DB rate of completely closed gaps was $1.4 \%$ ( 4 of 281): $5.9 \%$ (11 of 185) when clipping was partial (clips placed to achieve hemostasis of isolated vessels) and 3.9\% (30 of 775) when clips were not used. Twenty patients $(43.5 \%)$ required endoscopic treatment. A blood transfusion was necessary in 18 patients $(39.1 \%)$. No patients with DB underwent surgery and no mortality was reported.

Table 2 summarizes the univariate logistic regression analysis for possible DB predictors. Table 3 shows the multivariate analysis and the DB score assigned to each risk factor. Predictors significantly associated with DB were age 75 years or older (OR, 2.36; 95\% CI, 1.24-4.51), aspirin use during EMR (OR, 3.16; $95 \% \mathrm{CI}, 1.18-8.49$ ), location of the lesion proximal to the transverse colon (OR, 4.86; 95\% CI, 2.43-9.71), and not closing the mucosal gap with hemoclips (OR, 3.63; 95\% CI, 1.25-10.53). ASA classification of III or IV (OR, 1.90; 95\% CI, 0.99-3.64) and lesion size of $40 \mathrm{~mm}$ or larger (OR, 1.91; 95\% CI, 0.99-3.70) reached a $P$ value of .05 .

The DB total score ranged from 0 (lowest risk) to 10 (highest risk). The probability of DB depended on the score value and is detailed in Table 4 . The sample was classified into 3 risk categories: low (score, $0-3$ ), average (score, 4-7), and high (score, 8-10). This distribution had a ROC of $0.77(95 \% \mathrm{CI}, 0.70-0.83)$ and the probability of DB was $0.6 \%$ (95\% CI, $0.2 \%-1.8 \%), 5.5 \%$ (95\% CI, $3.8 \%-7.9 \%)$, and $40 \%$ (95\% CI, $21.8 \%-61.1 \%)$, respectively. The multivariate model had a ROC of 0.79 (95\% CI, 0.73-0.85) and, similarly, the score model had a ROC of 0.79 (95\% CI, 0.72-0.85), showing good discrimination ability. The bias-corrected index after bootstrap validation was 0.73 (95\% CI, 0.66-0.79). Calibration of the model also was good according to the calibration curves (Figure 1) and to the HosmerLemeshow results $\left(\chi^{2}=5.72 ; P=.572\right)$. The discrimination slope (difference in average predictions for those with and without the outcome) was 0.07 , with a 95\% CI of 0.04 to 0.1 and a $P$ value of less than .001 . Table 5 summarizes the score values in a practical table.

\section{Discussion}

In this multicenter prospective cohort we found that factors associated with DB were patient age 75 years and older, ASA classification of III or IV, aspirin use during 
Table 1. Descriptive Characteristics of the Sample: 1255 EMR in 1214 Patients

\begin{tabular}{|c|c|c|c|}
\hline Variable & $\mathrm{N}$ & Categories & $\mathrm{n}(\%)$ \\
\hline \multicolumn{4}{|l|}{ Demographic characteristics } \\
\hline Sex & 1214 & Female & $444(36.6 \%)$ \\
\hline Age, $y$ & 1214 & Mean (SD) & $67.9(10.9)$ \\
\hline \multirow[t]{2}{*}{ Age group } & \multirow[t]{2}{*}{1214} & $<75$ y & $843(69.4 \%)$ \\
\hline & & $\geq 75$ y & $371(30.6 \%)$ \\
\hline \multirow{3}{*}{ ASA classification } & \multirow{3}{*}{1242} & II & $650(52.3 \%)$ \\
\hline & & III & $345(27.8 \%)$ \\
\hline & & IV & $34(2.7 \%)$ \\
\hline \multirow[t]{3}{*}{ Aspirin treatment } & \multirow[t]{3}{*}{1242} & No & $1047(84.3 \%)$ \\
\hline & & Ceased before EMR & $144(11.5 \%)$ \\
\hline & & During EMR & $51(4.1 \%)$ \\
\hline Antiplatelet/anticoagulant therapy & 1241 & Dabigatran & $6(0.5 \%)$ \\
\hline \multirow[t]{6}{*}{ Paris classification } & \multirow[t]{6}{*}{1248} & Ols/Ols+0lla & $547(43.9 \%)$ \\
\hline & & Olla & $559(44.8 \%)$ \\
\hline & & Ollb & $88(7.1 \%)$ \\
\hline & & Ollc & $10(0.8 \%)$ \\
\hline & & Olla+0llc & $38(3 \%)$ \\
\hline & & Ollb+0llc & $6(0.5 \%)$ \\
\hline Lesion size, $\mathrm{mm}$ & 1255 & Mean (SD) & $30.5(11.8)$ \\
\hline \multirow[t]{2}{*}{ Location proximal to splenic flexure } & \multirow[t]{2}{*}{1253} & No & $610(48.7 \%)$ \\
\hline & & Yes & $643(51.3 \%)$ \\
\hline Location proximal to transverse & 1253 & No & $752(60.0 \%)$ \\
\hline & & Yes & $501(40.0 \%)$ \\
\hline & & En bloc & $304(24.6 \%)$ \\
\hline Adrenaline use for submucosal injection & 1247 & No & $406(32.6 \%)$ \\
\hline & & Yes & $841(67.4 \%)$ \\
\hline Electrocautery current used & 1247 & Cut current & $19(1.5 \%)$ \\
\hline & & Coagulation current & $172(13.8 \%)$ \\
\hline & & Blended (endocut mode) & $1056(84.7 \%)$ \\
\hline APC for coagulation & 1247 & No & $1163(93.3 \%)$ \\
\hline & & Yes & $84(6.7 \%)$ \\
\hline Clipping & 1241 & No & $775(62.4 \%)$ \\
\hline & & Partially clipped & $185(14.9 \%)$ \\
\hline & & Fully clipped & $281(22.6 \%)$ \\
\hline Complete closure & 1241 & No & $960(77.4 \%)$ \\
\hline & & Yes & $281(22.6 \%)$ \\
\hline Adverse events & & & \\
\hline Delayed bleeding & 1247 & Yes & $46(3.7 \%)$ \\
\hline & & $<24 \mathrm{~h}$ & $13(28.3 \%)$ \\
\hline & & $24-48 \mathrm{~h}$ & $4(8.7 \%)$ \\
\hline & & $3-7 d$ & $23(50 \%)$ \\
\hline & & $>7 \mathrm{~d}$ & $6(13 \%)$ \\
\hline Immediate bleeding & 1247 & Yes & $66(5.3 \%)$ \\
\hline Perforation & 1245 & No & $1228(98.6 \%)$ \\
\hline & & Yes: endoscopic resolution & $16(1.3 \%)$ \\
\hline & & Yes: surgical resolution & $1(0.1 \%)$ \\
\hline Deaths & 1255 & No & $1255(100 \%)$ \\
\hline
\end{tabular}

$\mathrm{APC}$, argon plasma coagulation; HGD, high-grade dysplasia. 
Table 2. Analysis of Factors Possibly Related to DB After EMR

\begin{tabular}{|c|c|c|c|c|}
\hline Variable & Categories & OR $(95 \% \mathrm{Cl})$ & $P$ & $\mathrm{R}^{2}$ Nagelkerke \\
\hline \multirow[t]{2}{*}{ Sex } & Female & Reference & & \\
\hline & Male & $1.30(0.68-2.47)$ & .425 & 0.002 \\
\hline \multirow[t]{2}{*}{ Age group } & $<75$ y & Reference & & \\
\hline & $\geq 75$ y & $2.98(1.63-5.43)$ & $<.001$ & 0.038 \\
\hline ASA & $|-| \mid$ & Reference & & \\
\hline classification & III-IV & 2.57 (1.42-4.64) & .002 & 0.028 \\
\hline Aspirin use & No & Reference & & \\
\hline during EMR & Yes & $1.95(0.99-3.84)$ & .053 & 0.010 \\
\hline \multirow[t]{5}{*}{ Antiplatelet/anticoagulant therapy } & No & Reference & & \\
\hline & Antiplatelet & $2.79(1.41-5.51)$ & & \\
\hline & Dual- antiplatelet & $12.87(2.46-67.2)$ & $<.001$ & 0.073 \\
\hline & Warfarin/acenocumarol & $1.61(0.54-4.75)$ & & \\
\hline & Dabigatran & 38.6 (7.39-201.6) & & \\
\hline \multirow[t]{2}{*}{ Paris classification } & Not Ols, Ols-0lla & Reference & & \\
\hline & Ols, Ols-0lla & $0.67(0.36-1.25)$ & .212 & 0.005 \\
\hline \multirow[t]{2}{*}{ Proximal to splenic flexure } & No & Reference & & \\
\hline & Yes & 3.55 (1.74-7.21) & $<.001$ & 0.043 \\
\hline \multirow[t]{2}{*}{ Proximal to transverse colon } & No & Reference & & \\
\hline & Yes & $4.49(2.30-8.76)$ & $<.001$ & 0.066 \\
\hline \multirow{2}{*}{ Size, $m m$} & $<40$ & Reference & & \\
\hline & $\geq 40$ & $1.81(0.97-3.37)$ & .061 & 0.010 \\
\hline \multirow[t]{2}{*}{ Histology } & Serrated & Reference & & \\
\hline & Adenoma & $2.63(0.63-11.00)$ & .184 & 0.003 \\
\hline \multirow[t]{2}{*}{ Resection } & Piecemeal & Reference & & \\
\hline & En bloc & $0.45(0.19-1.08)$ & .073 & 0.011 \\
\hline \multirow[t]{2}{*}{ Adrenaline use } & No & Reference & & \\
\hline & Yes & 0.67 (0.37-1.22) & .192 & 0.005 \\
\hline \multirow[t]{2}{*}{ APC for coagulation } & No & Reference & & \\
\hline & Yes & $0.73(0.26-2.01)$ & .564 & 0.001 \\
\hline \multirow[t]{3}{*}{ Clipping } & No & Reference & & \\
\hline & Partially clipped & $1.59(0.78-3.23)$ & .041 & 0.023 \\
\hline & Fully clipped & $0.36(0.12-1.02)$ & & \\
\hline \multirow[t]{2}{*}{ Complete closure } & Yes & Reference & & \\
\hline & No & $3.11(1.11-8.77)$ & .032 & 0.019 \\
\hline
\end{tabular}

EMR, location of the lesion proximal to the transverse colon, a lesion size of $40 \mathrm{~mm}$ or larger, and no complete mucosal closure with clips. On the basis of these risk factors, we developed a DB scoring system for EMR of large colorectal lesions.

The risk factors we found to be associated with DB have been described in other observational studies, but most of them still are under discussion. Most series have found that lesions located in the right $\operatorname{colon}^{5,8,20,25,26}$ and a large polyp size $e^{4,8,20,25-28}$ are related to a greater risk of DB, but other factors such as age, ${ }^{5,29,30}$ aspirin or nonsteroidal anti-inflammatory drug use, ${ }^{4,5,27,30-32}$ hypertension, ${ }^{4,28}$ anticoagulant therapy, ${ }^{4,30}$ or bleeding during the $\mathrm{EMR}^{2,24}$ remain controversial.

Age older than 75 years (30.6\% in our cohort) was associated with DB. Metz et $\mathrm{al}^{5}$ found an OR of 1.7 per decade of age. This was an important finding because this population group is increasing, and adverse events in older patients might have more clinical impact.

Aspirin was associated with an increased prevalence of postpolypectomy bleeding in 2 large studies. ${ }^{5,32}$ In our series, the DB rate in the 50 patients $(4.1 \%)$ who had asnirin treatment during EMR was $12 \%$, and aspirin therapy during EMR added 2 points to our DB risk score, whereas other large observational studies did not find aspirin therapy was a risk factor. ${ }^{6,30,31}$

Our findings regarding lesion location and size association with DB are well supported in the literature. First, the proximal colonic location of the lesion clearly is associated with DB in many studies, ${ }^{5,8,20,25,26}$ including a randomized controlled trial of 347 EMRs of large colorectal nonpedunculated lesions ${ }^{13}$ and a large observational study of 1172 patients. ${ }^{2}$ It was the most influential risk factor in our study with the highest weight in the score. Second, lesion size also seems to be associated with DB in the literature, ${ }^{4,6,8,28,29}$ although not in all studies. $^{2,33}$

Several prophylactic methods have been described to prevent DB. Adding adrenaline to the submucosal injection has a preventive effect on immediate bleeding, ${ }^{34}$ but it is not useful for $\mathrm{DB}^{9-11}$ or in our study. Other techniques empirically used such as argon plasma coagulation and coagulation forceps found no preventive effects in 2 randomized controlled trials, ${ }^{12,13}$ as in our series. Another prophylactic approach could be to place hemoclips on visible vessels or to close the mucosal defects. 
Table 3. Multivariate Logistic Regression Model and Independent Predictors of DB After EMR

\begin{tabular}{|c|c|c|c|c|c|}
\hline Variable & Categories & Adjusted OR $(95 \% \mathrm{Cl})$ & $P$ & $\beta$ & Score value \\
\hline \multirow[t]{2}{*}{ Age group } & $<75$ y & Reference & & & 0 \\
\hline & $\geq 75$ y & $2.36(1.24-4.51)$ & .009 & 0.860 & 1 \\
\hline \multirow[t]{2}{*}{ ASA status } & I-II & Reference & & & 0 \\
\hline & III-IV & $1.90(0.99-3.64)$ & .052 & 0.644 & 1 \\
\hline \multirow[t]{2}{*}{ Size, $m m$} & $<40$ & Reference & & & 0 \\
\hline & $\geq 40$ & $1.91(0.99-3.70)$ & .055 & 0.647 & 1 \\
\hline \multirow[t]{2}{*}{ Aspirin use } & No & Reference & & & 0 \\
\hline & Yes & $3.16(1.18-8.49)$ & .022 & 1.152 & 2 \\
\hline \multirow{2}{*}{ Complete closure } & Yes & Reference & & & 0 \\
\hline & No & $3.63(1.25-10.53)$ & .018 & 1.288 & 2 \\
\hline \multirow[t]{2}{*}{ Proximal to transverse } & No & Reference & & & 0 \\
\hline & Yes & $4.86(2.43-9.71)$ & $<.001$ & 1.580 & 3 \\
\hline
\end{tabular}

NOTE. The ROC was 0.79 , and the $95 \% \mathrm{Cl}$ was 0.73 to 0.85 .

In a retrospective study of 524 large colonic lesions, the DB rate was $9.7 \%$ in the not clipped group vs $1.8 \%$ in the fully clipped group (OR, 6.0; 95\% CI, 2.0-18.5). ${ }^{20} \mathrm{~A}$ recent prospective controlled study randomly assigned patients to a clip-closure or a no-clip closure group after EMR and endoscopic submucosal resection of colorectal lesions, and the DB rate was lower in the clip-closure group $(1.1 \%$ vs $6.9 \% ; P<.01) .{ }^{21}$ In our cohort, the DB rate of completely closed gaps was reduced significantly. Nevertheless, in a randomized controlled trial, clipping did not reduce DB after EMR in lesions with a mean size of less than $10 \mathrm{~mm} .^{16}$ This simply may be because small lesions have a lower risk of DB. Despite the possible benefits of hemoclips, they have several limitations. Closing large or difficult-to-access mucosal defects may be challenging and the number of clips needed might increase procedure costs, as reported in a study evaluating prophylactic clipping of all large colorectal lesions after EMR. ${ }^{35}$ Another limitation could be the risk of submucosal recurrence owing to residual pathologic tissue after EMR.

Considering this DB risk score, patients can be classified into 3 categories (low, average, and high) according to a numeric scale. Modifiable factors such as the use of hemoclips and aspirin withdrawal before and/or after

Table 4. Probability of DB Depending on the Score Value

\begin{tabular}{lcc}
\hline Score & DB risk & DB risk category \\
\hline 0 & $0 \%$ & Low \\
1 & $0 \%$ & $\mathrm{n}=618$ \\
2 & $1 \%$ & $(0.6 \%)$ \\
3 & $1 \%$ & \\
4 & $2 \%$ & Average \\
5 & $4 \%$ & $\mathrm{n}=541$ \\
6 & $8 \%$ & $(5.5 \%)$ \\
7 & $13 \%$ & \\
8 & $22 \%$ & $\mathrm{High}$ \\
9 & $34 \%$ & $\mathrm{n}=25$ \\
10 & $48 \%$ & $(40 \%)$
\end{tabular}

the EMR, together accounting for 4 points, can reduce the estimated risk of bleeding, reducing the need for a more expensive hospitalization, blood transfusion, and repetition of endoscopies in the high-risk DB patients. On the other hand, the remaining nonmodifiable variables (patient's age, ASA classification, polyp size, and right location) are easily recognizable by the endoscopist and the clinician. The sum of all these factors might determine the need for prophylactic measures or inpatient observation in selected patients.

The main strengths of the study were the large series and the participation of multiple endoscopists and institutions. Although resection methods may have varied considerably between endoscopy units, our findings reflect daily clinical practice in different settings. In addition, we provide a tool to identify patients at high risk of DB who may benefit from prophylactic measures.

The major limitation, also associated with the multicenter and observational nature of the study, was the possible selection bias and some cases might have been under-reported. However, all participating doctors included at least 20 patients and their participation was

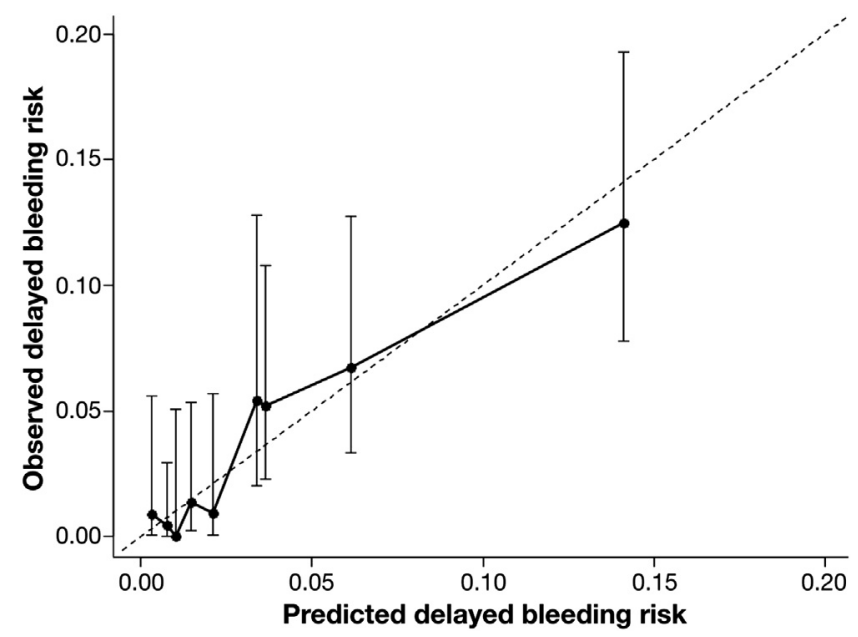

Figure 1. Calibration curve for the multivariate regression model. 
Table 5. Score Values of the DB Risk Factors

\begin{tabular}{lc}
\hline \multicolumn{1}{c}{ Variable } & Score \\
\hline Age $\geq 75$ y & 1 \\
ASA class, III-IV & 1 \\
Lesion size $\geq 40 \mathrm{~mm}$ & 1 \\
Aspirin treatment & 2 \\
No complete closure after EMR & 2 \\
Lesion location proximal to transverse & 3
\end{tabular}

NOTE. Total score of 0-3: low risk of DB $(0.6 \%$; $95 \% \mathrm{Cl}, 0.2-1.8)$; score of $4-7$ : average risk of DB (5.5\%; $95 \% \mathrm{Cl}, 3.8-7.9)$; or score of $8-10$ : high risk of DB (40\%; 95\% Cl, 21.8-61.1).

stable during the study period. Some factors such as exact power cautery settings were not recorded prospectively because of their variability. This might be relevant because a study by Liaquat et $\mathrm{al}^{20}$ that used lowpower coagulation described a $9.7 \%$ DB rate in the notclipped group. In addition, the Australian group mentioned that the use of Endocut was associated with a lower risk of bleeding compared with low-power coagulation. $^{2,5}$ In our series, the electrocautery current used, mostly Endocut, was not associated with significant statistical differences in DB rate, but the study was not designed for this evaluation. Another limitation was the relatively low incidence of DB of $3.7 \%$ because most recent series reported an incidence closer to $6 \%$ to $7 \%$. This difference could be related to the fact that almost a quarter of the lesions were completely closed or to the use of Endocut as cautery. Nevertheless, one recent meta-analysis reported an incidence of $2.6 \%$, although the data were evaluable in only 32 of 50 studies. $^{3}$ Because of this low bleeding risk, the predictive score was derived from the whole cohort, hence an external validation could not be conducted. Although we minimized this problem by using a resampling method, an external validation in subsequent studies was necessary to confirm and refine this score.

\section{Conclusions}

In this large, prospective, multicenter series, 6 simple variables (age, $\geq 75$ y; ASA classification III or IV, aspirin use during EMR, location of lesion proximal to the transverse colon, lesion size $\geq 40 \mathrm{~mm}$, and no mucosal complete closure with clips) were associated with DB after EMR. We developed a predictive score to estimate the $\mathrm{DB}$ risk that could be used in the management of these patients to guide the prophylactic treatment and observation period. It also might be useful in clinical trials to evaluate prevention strategies.

\section{References}

1. Othman MO, Wallace MB. Endoscopic mucosal resection (EMR) and endoscopic submucosal dissection (ESD) in 2011, a Western perspective. Clin Res Hepatol Gastroenterol 2011;35:288-294.

2. Burgess NG, Metz AJ, Williams SJ, et al. Risk factors for intraprocedural and clinically significant delayed bleeding after wide-field endoscopic mucosal resection of large colonic lesions. Clin Gastroenterol Hepatol 2014;12:651-661.

3. Hassan C, Repici A, Sharma P, et al. Efficacy and safety of endoscopic resection of large colorectal polyps: a systematic review and meta-analysis. Gut 2016;65:806-820.

4. Sawhney MS, Salfiti N, Nelson DB, et al. Risk factors for severe delayed postpolypectomy bleeding. Endoscopy 2008; 40:115-119.

5. Metz AJ, Bourke MJ, Moss A, et al. Factors that predict bleeding following endoscopic mucosal resection of large colonic lesions. Endoscopy 2011;43:506-511.

6. Qumseya BJ, Wolfsen C, Wang Y, et al. Factors associated with increased bleeding post-endoscopic mucosal resection. J Dig Dis 2013;14:140-146.

7. Wu XR, Church JM, Jarrar A, et al. Risk factors for delayed postpolypectomy bleeding: how to minimize your patients' risk. Int J Colorectal Dis 2013;28:1127-1134.

8. Kim JH, Lee HJ, Ahn JW, et al. Risk factors for delayed postpolypectomy hemorrhage: a case-control study. J Gastroenterol Hepatol 2013;28:645-649.

9. Hsieh YH, Lin HJ, Tseng GY, et al. Is submucosal epinephrine injection necessary before polypectomy? A prospective, comparative study. Hepatogastroenterology 2001; 48:1379-1382.

10. Dobrowolski S, Dobosz M, Babicki A, et al. Prophylactic submucosal saline-adrenaline injection in colonoscopic polypectomy: prospective randomized study. Surg Endosc 2004; 18:990-993.

11. Lee SH, Chung IK, Kim SJ, et al. Comparison of postpolypectomy bleeding between epinephrine and saline submucosal injection for large colon polyps by conventional polypectomy: a prospective randomized, multicenter study. World J Gastroenterol 2007;13:2973-2977.

12. Lee CK, Lee SH, Park JY, et al. Prophylactic argon plasma coagulation ablation does not decrease delayed postpolypectomy bleeding. Gastrointest Endosc 2009;70:353-361.

13. Bahin FF, Naidoo M, Williams SJ, et al. Prophylactic endoscopic coagulation to prevent bleeding after wide-field endoscopic mucosal resection of large sessile colon polyps. Clin Gastroenterol Hepatol 2015;13:724-730.

14. Parra-Blanco A, Kaminaga N, Kojima T, et al. Hemoclipping for postpolypectomy and postbiopsy colonic bleeding. Gastrointest Endosc 2000;51:37-41.

15. Sobrino-Faya M, Martínez S, Gómez-Balado M, et al. Clips for the prevention and treatment of postpolypectomy bleeding (hemoclips in polypectomy). Rev Esp Enferm Dig 2002; 94:457-462.

16. Shioji K, Suzuki Y, Kobayashi M, et al. Prophylactic clip application does not decrease delayed bleeding after colonoscopic polypectomy. Gastrointest Endosc 2003;57:691-694.

17. Lee BI, Choi KY, Kim BW, et al. Approximation of large mucosal defects after endoscopic mucosal resection: a new technique using a detachable snare and clips. Gastrointest Endosc 2004; 59:P150.

18. Endo M, Inomata M, Terui T, et al. New endoscopic technique to close large mucosal defects after endoscopic mucosal resection in patients with gastric mucosal tumors. Dig Endosc 2004; 16:327-335.

19. Hokama A, Kishimoto K, Kinjo F, et al. Endoscopic clipping in the lower gastrointestinal tract. World J Gastrointest Endosc 2009;15:7-11. 
20. Liaquat H, Rohn E, Rex DK. Prophylactic clip closure reduced the risk of delayed postpolypectomy hemorrhage: experience in 277 clipped large sessile or flat colorectal lesions and 247 control lesions. Gastrointest Endosc 2013;77:401-407.

21. Zhang QS, Han B, Xu JH, et al. Clip closure of defect after endoscopic resection in patients with larger colorectal tumors decreased the adverse events. Gastrointest Endosc 2015; 82:904-909.

22. Bourke M. Endoscopic mucosal resection in the colon: a practical guide. Tech Gastrointest Endosc 2011;13:35-49.

23. Sakamoto N, Beppu K, Matsumoto K, et al. "Loop Clip", a new closure device for large mucosal defects after EMR and ESD. Endoscopy 2008;40(Suppl 2):E97-E98.

24. Kim HS, Kim TI, Kim WH, et al. Risk factors for immediate postpolypectomy bleeding of the colon: a multicenter study. Am J Gastroenterol 2006;101:1333-1341.

25. Buddingh $\mathrm{KT}$, Herngreen T, Haringsma J, et al. Location in the right hemi-colon is an independent risk factor for delayed postpolypectomy hemorrhage: a multi-center case-control study. Am J Gastroenterol 2011;106:1119-1124.

26. Albéniz E, Fraile M, Martínez-Ares D, et al. Delayed bleeding after endoscopic mucosal resection of large colorectal lesions. A prospective multicenter Spanish study. Gastrointest Endosc 2015;79:AB438-AB439.

27. Shiffman ML, Farrel MT, Yee YS. Risk of bleeding after endoscopic biopsy or polypectomy in patients taking aspirin or other NSAIDS. Gastrointest Endosc 1994;40:458-462.

28. Watabe H, Yamaji $\mathrm{Y}$, Okamoto M, et al. Risk assessment for delayed hemorrhagic complication of colonic polypectomy: polyp-related factors and patient-related factors. Gastrointest Endosc 2006;64:73-78.

29. Sorbi D, Norton I, Conio M, et al. Postpolypectomy lower GI bleeding: descriptive analysis. Gastrointest Endosc 2000; 51:690-696.
30. Hui AJ, Wong RM, Ching JY, et al. Risk of colonoscopic polypectomy bleeding with anticoagulants and antiplatelet agents: analysis of 1657 cases. Gastrointest Endosc 2004; 59:44-48.

31. Manocha D, Singh M, Mehta N, et al. Bleeding risk after invasive procedures in aspirin/NSAID users: polypectomy study in veterans. Am J Med 2012;125:1222-1227.

32. Pan A, Schlup M, Lubcke $R$, et al. The role of aspirin in postpolypectomy bleeding-a retrospective survey. BMC Gastroenterol 2012;12:138.

33. Buchner AM, Guarner-Argente C, Ginsberg GG. Outcomes of EMR of defiant colorectal lesions directed to an endoscopy referral center. Gastrointest Endosc 2012;76:255-263.

34. Li LY, Liu QS, Li L, et al. A meta-analysis and systematic review of prophylactic endoscopic treatments for postpolypectomy bleeding. Int J Colorectal Dis 2011;26:709-719.

35. Bahin FF, Rasouli KN, Williams SJ, et al. A prophylactic clip strategy is not cost effective for the prevention of clinically significant bleeding following wide-field endoscopic mucosal resection of large colorectal sessile and laterally spreading lesions. Gastrointest Endosc 2015;81:AB134.

\section{Reprint requests}

Address requests for reprints to: Eduardo Albéniz, MD, Endoscopy Unit, Gastroenterology Department, Complejo Hospitalario de Navarra, Pamplona, Irunlarrea 3, 31008, Navarra, Spain. e-mail: edualbeniz@hotmail.com.

\section{Acknowledgments}

The authors would like to acknowledge Carolyn Newey for help in editing the manuscript and Navarrabiomed-Fundación Miguel Servet for their support with the statistical analysis.

Conflicts of interest

The authors disclose no conflicts. 


\section{Clinical validation of risk scoring systems to predict risk of delayed bleeding after EMR of large colorectal lesions CME}

Eduardo Albéniz, MD, ${ }^{1}$ Antonio Zebenzuy Gimeno-García, MD, PhD, ${ }^{2}$ María Fraile, MD ${ }^{3}$ Berta Ibánez, PhD, ${ }^{4}$ Carlos Guarner-Argente, MD, ${ }^{5}$ Pedro Alonso-Aguirre, MD, ${ }^{6}$ Marco Antonio Álvarez, MD, Carla Jerusalén Gargallo, MD,${ }^{8}$ María Pellisé, MD, PhD, ${ }^{9}$ Felipe Ramos Zabala, MD, ${ }^{10}$ Alberto Herreros de Tejada, MD, PhD, ${ }^{11}$ Óscar Nogales, MD, ${ }^{12}$ David Martínez-Ares, MD, ${ }^{13}$ Fernando Múgica, MD, ${ }^{14}$ Joaquín de la Peña, MD,${ }^{15}$ Jorge Espinós, MD, ${ }^{16}$ Alain Huerta, MD, ${ }^{17}$ Alberto Álvarez, MD, ${ }^{18}$ Jesús M. Gonzalez-Santiago, MD, ${ }^{18}$ Francisco Navajas, MD, ${ }^{19}$ Juan Gabriel Martínez-Cara, MD ${ }^{20}$ Eduardo Redondo-Cerezo, MD, ${ }^{20}$ Josep Merlo Mas, MD, ${ }^{21}$ Fernando Sábado, MD, ${ }^{22}$ Liseth Rivero, MD, ${ }^{9}$ Esteban Saperas, MD, ${ }^{23}$ Santiago Soto, MD, ${ }^{24}$ Joaquín Rodríguez-Sánchez, MD ${ }^{25}$ Leopoldo López-Roses, MD, ${ }^{26}$ Manuel Rodríguez-Téllez, MD, ${ }^{27}$ María Rullán Iriarte, MD, ${ }^{1}$ Alfonso Elosua González, MD, ${ }^{1}$ Remedios Pardeiro, MD, ${ }^{6}$ Eduardo Valdivielso Cortázar, MD, ${ }^{6}$ Mar Concepción-Martín, MD, ${ }^{5}$ Patricia Huelin Álvarez, MD, ${ }^{5}$ Juan Colán Hernández, MD, Julyssa Cobian, MD, ${ }^{14}$ José Santiago, MD, ${ }^{11}$ Alejandra Jiménez, MD, ${ }^{27}$ David Remedios, MD, ${ }^{24}$ Bartolomé López-Viedma, MD, ${ }^{25}$ Orlando García, MD, ${ }^{28}$ Felipe Martínez-Alcalá, MD ${ }^{29}$ Francisco Pérez-Roldán, MD,${ }^{30}$ Jorge Carbó, MD,${ }^{31}$ Mónica Enguita, PhD ${ }^{32}$

Pamplona, Tenerife, Logroño, Barcelona, La Coruña, Zaragoza, Madrid, Vigo, Donostia-San Sebastián, Santander, Galdakao, Salamanca, Toledo, Granada, Castellón, Ourense, Ciudad Real, Lugo, Sevilla, Sant Joan Despí, Spain

\section{GRAPHICAL ABSTRACT}

Multicenter cohort studv: 1034 EMRs of large non-pedunculated colorectal polyps (LNPCL) were included.

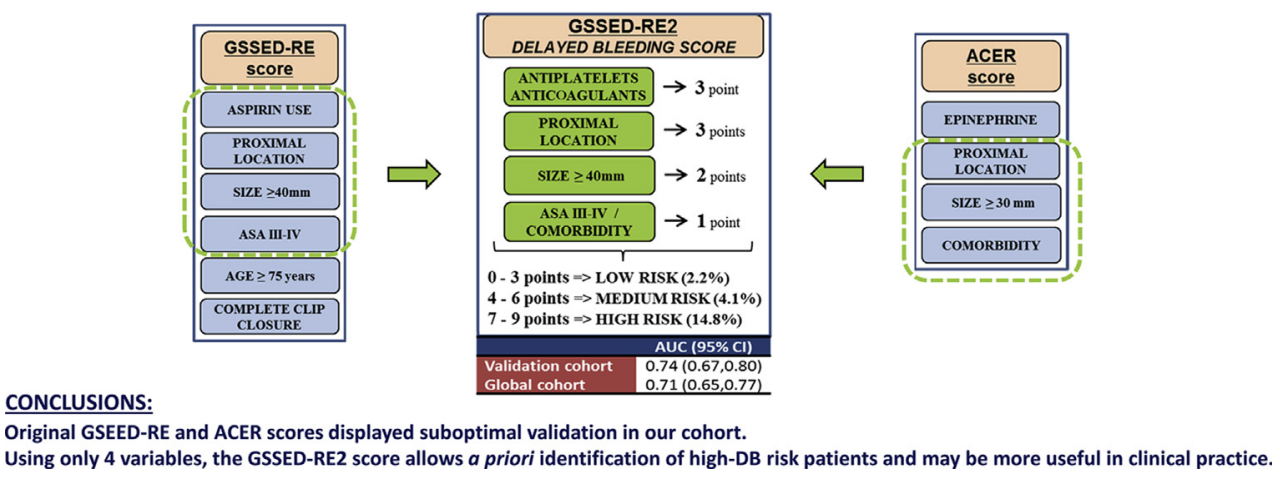

Background and Aims: The Endoscopic Resection Group of the Spanish Society of Endoscopy (GSEED-RE) model and the Australian Colonic Endoscopic Resection (ACER) model were proposed to predict delayed bleeding (DB) after EMR of large superficial colorectal lesions, but neither has been validated. We validated and updated these models.

Methods: A multicenter cohort study was performed in patients with nonpedunculated lesions $\geq 20$ mm removed by EMR. We assessed the discrimination and calibration of the GSEED-RE and ACER models. Difficulty performing EMR was subjectively categorized as low, medium, or high. We created a new model, including factors associated with DB in 3 cohort studies.

Results: DB occurred in 45 of 1034 EMRs (4.5\%); it was associated with proximal location (odds ratio [OR], 2.84; 95\% confidence interval [CI], 1.31-6.16), antiplatelet agents (OR, 2.51; 95\% CI, .99-6.34) or anticoagulants (OR, 4.54; 95\% CI, 2.14-9.63), difficulty of EMR (OR, 3.23; 95\% CI, 1.41-7.40), and comorbidity (OR, 2.11; 95\% CI, 99-4.47). The GSEED-RE and ACER models did not accurately predict DB. Re-estimation and recalibration yielded acceptable results (GSEED-RE area under the curve [AUC], .64 [95\% CI, .54-.74]; ACER AUC, .65 [95\% CI, .57.73]). We used lesion size, proximal location, comorbidity, and antiplatelet or anticoagulant therapy to generate 
a new model, the GSEED-RE2, which achieved higher AUC values (.69-.73; 95\% CI, .59-.80) and exhibited lower susceptibility to changes among datasets.

Conclusions: The updated GSEED-RE and ACER models achieved acceptable prediction levels of DB. The GSEED-RE2 model may achieve better prediction results and could be used to guide the management of patients after validation by other external groups. (Clinical trial registration number: NCT 03050333.) (Gastrointest Endosc 2020;91:868-78.)

(footnotes appear on last page of article)

EMR is the standard procedure in cases of large superficial colorectal lesions in Western countries and is an effective treatment for the prevention of colorectal cancer. ${ }^{-}$ Delayed bleeding (DB) is the most frequent major adverse event, with an incidence of $2.6 \%$ to $9.7 \%$ for colorectal lesions larger than $2 \mathrm{~cm}^{2,3}$ Large lesions and proximal colon location are risk factors for DB. However, the role of other parameters, such as age ${ }^{4-6}$ ingestion of aspirin or nonsteroidal anti-inflammatory drugs, ${ }^{6-10}$ hypertension, ${ }^{6,11}$ anticoagulant therapy, ${ }^{6,7}$ and bleeding during EMR, ${ }^{2,12}$ are controversial. Despite the non-negligible incidence of DB, no generally accepted guidelines define which patients benefit the most from DB preventive measures.

Two models were designed to predict DB. The Endoscopic Resection Group of the Spanish Society of Endoscopy (GSEED-RE) ${ }^{13}$ designed a scoring system that includes the following risk variables from lowest to highest: age, American Society of Anesthesiologists (ASA) risk classification, aspirin use, lesion size, complete clip closure of post-EMR defects, and proximal location. In the second model, the Australian Colonic Endoscopic Resection ACER) study risk score ${ }^{14}$ the absence of adrenaline in the injection solution, major comorbidities, lesion size, and proximal location were used as independent risk factors from lowest to highest. Although both series included a large number of patients, the independent variables resulting from these studies do not tally, and neither model was validated by other working groups.

In the present study we aim to validate the GSEED-RE and ACER DB scoring models. Secondary aims were to update these models and develop a new, improved model.

\section{METHODS}

\section{Patients}

Twenty-six GSEED-RE endoscopists at 23 Spanish hospitals began a multicenter prospective observational study in April 2013. Nonpedunculated colorectal lesions $\geq 2 \mathrm{~cm}$ that underwent EMR were consecutively recorded in a centralized database. Endoscopists participating in the study performed a minimum of 20 large resections per year. All patients who had indications for EMR of colorectal lesions were screened for inclusion. Subjects who refused to participate in the study were excluded. Patient characteristics, lesion features, and the EMR techniques used were collected using a standardized data recording form (Table 1).

A major comorbidity was defined as a chronic systemic condition that substantially impaired patient functioning and required treatment. ${ }^{14}$ Comorbidities included in the Charlson comorbidity index were considered major comorbidities for this study. ${ }^{15}$ The difficulty of EMR was subjectively rated as low, medium, or high depending on the location (eg, location behind folds and lesions of the ileocecal valve were deemed difficult locations), type (eg, nongranular pseudodepressed lateral spreading tumors were deemed difficult lesions), and size of the lesions $(>30 \mathrm{~mm}){ }^{16}$ Therefore, low difficulty was assigned if the location, type, and size were favorable for resection, and high difficulty was considered when at least 2 of the 3 criteria were unfavorable for resection. The GSEED-RE (the first model of the Spanish group) ${ }^{13}$ was applied to patients until February 2015 (pre-2015 cohort). Patients included between March 2015 and November 2016 constituted the test cohort (post-2015 cohort).

Participating patients gave written informed consent for their inclusion in the study. The Ethics Committee approved the study protocol (ALB-RME-2013-01/ NCT03050333) in February 2013. The first patient was included in April 2013 and the last patient in November 2016. All authors had access to the study data and reviewed and approved the final manuscript.

\section{EMR technique and special conditions}

Experienced senior endoscopists performed the procedures using conventional or high-definition white-light endoscopes. Injection-assisted EMR was performed as described elsewhere. ${ }^{17}$ Lesion size was measured as the longitudinal size of an open snare. The mucosal defect was closed with hemoclips at the discretion of the endoscopist. ${ }^{18}$ Complete closure was defined when the clips were applied next to each other and there were no substantial submucosal areas in the closure line. Closure was considered partial when it was not completely closed because of a large size or complex location. Punctual clipping was defined as the placement of the clip only on solated vessels (cut vessels, oozing vessels). No standardized electrocautery current was applied. Blended current or the endocut mode were primarily used. 


\section{TABLE 1. Descriptive characteristics of the post-2015 cohort (1034 EMRs in 977 patients)}

\section{Variable}

Demographic characteristics (977 patients)

Sex

Age, $y$

Age group

Clinical characteristics (1034 EMRs)

ASA classification

Comorbidity

\section{Paris classification}

Lesion size, $\mathrm{mm}$

Lesion size $<40 \mathrm{~mm}$

Lesion size $\geq 40 \mathrm{~mm}$

Location proximal to the splenic flexure (not included)

Location proximal to the hepatic flexure (not included)

Histology

EMR characteristics
Resection

Adrenaline use for submucosal injection

\section{8}

973

\section{No. of cases}

975

975

975

890

923

951

1014

1031

1034

1034

999

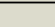

Categories

n (\%)*

975

Female

Male

Mean (SD)

$<75$ y

$\geq 75 \mathrm{y}$

$70(37.9)$

$605(62.1)$

$68.1(10.4)$

$689(70.7)$

$286(29.3)$

\section{I}

$214(24.0)$

II

460 (51.7)

III

201 (22.6)

IV

$15(1.7)$

No

$336(36.4)$

Yes

587 (63.6)

None

$703(68.0)$

None
Aspirin ceased before EMR
Aspirin during EMR
Clopidogrel

$83(8.0)$

$43(4.2)$

$14(1.4)$

Aspirin + clopidogrel

$9(.9)$

Other antiplatelet

$11(1.1)$

Warfarin/acenocumarol

New anticoagulants (dabigatran)

$76(7.4)$

Antiplatelet+ (anticoagulants)

$4(.4)$

8 (.8)

\begin{tabular}{c} 
Ols \\
\hline Olla \\
\hline Ollb \\
\hline Ollc \\
Olla+0llc \\
Ollb+0llc \\
Mean (SD)
\end{tabular}

Yes

HGD-intramucosal carcinoma $295(29.5)$ Invasive carcinoma 49 (4.9)

$$
\text { Serrated }
$$

HGD-intramucosal carcinoma serrated

Invasive carcinoma serrated

Piecemea
En bloc
No




\begin{tabular}{|c|c|c|c|}
\hline & & Yes & $654(63.2)$ \\
\hline \multirow[t]{3}{*}{ Electrocautery current used } & 972 & Cut current & $2(.2)$ \\
\hline & & Coagulation current & $103(10.6)$ \\
\hline & & Blended (endocut mode) & $867(89.2)$ \\
\hline APC for coagulation (argon) & & Yes & $49(4.7)$ \\
\hline \multirow[t]{3}{*}{ Clipping } & 972 & No & $562(57.8)$ \\
\hline & & Partially clipped & $178(18.3)$ \\
\hline & & Fully clipped & $232(23.9)$ \\
\hline \multirow[t]{6}{*}{ Delayed bleeding } & 1010 & No & $965(95.5)$ \\
\hline & & Yes & $45(4.5)$ \\
\hline & & $<24 \mathrm{~h}$ & $8(.8)$ \\
\hline & & $24-48 \mathrm{~h}$ & $9(.9)$ \\
\hline & & $3-7 d$ & $18(1.7)$ \\
\hline & & $>7 d$ & $10(1.0)$ \\
\hline \multirow[t]{2}{*}{ Immediate bleeding } & 1008 & No & $951(94.2)$ \\
\hline & & Yes & $59(5.8)$ \\
\hline Perforation & 1017 & No & $998(98.1)$ \\
\hline
\end{tabular}

$S D$, Standard deviation; ASA, American Society of Anesthesiologists; $H G D$, high-grade dysplasia; $A P C$, argon plasma coagulation.

*Other values defined.

Anticoagulation and antiplatelet management was uniformly used following available guidelines. ${ }^{19}$ Traditional oral anticoagulants were withdrawn 5 days before the procedure, and bridging therapy with low-molecular-weight heparin was established until the expected anticoagulation level was recovered. Before the procedure, an international normalized ratio $<1.5$ was confirmed in patients taking anticoagulation therapy. Warfarin was restarted the day of the procedure. Direct oral anticoagulants were withdrawn 2 to 3 days before the procedure and restarted the day after the procedure. Aspirin cessation depended on comorbidities. Thienopyridines were routinely withdrawn 5 days before $\mathrm{EMR}^{19}$ and reintroduced the day after the procedure.

Participants were contacted 15 to 30 days after EMR. Follow-up was performed as an outpatient service at each center.

\section{Definitions and outcomes}

DB after EMR was defined as a clinically evident bleeding that required medical attention ( 2 of the following 3 parameters after complete polypectomy: gross hematochezia or dizziness, hemoglobin loss $>2 \mathrm{~g} / \mathrm{dL}$, and blood pressure decrease $>20 \mathrm{~mm} \mathrm{Hg}$ or a pulse rate increase $>20 \%$ of baseline) within 15 days of the procedure. $^{12}$ The primary goal was validation of the GSEED-RE and ACER models and identification of the risk factors associated with DB after EMR of large nonpedunculated colorectal lesions. Secondary aims were to update these models and develop a new improved model.

\section{Statistical analyses}

Characteristics of the post-2015 cohort are presented as frequencies (percentages) and means (standard deviations) and are compared against the characteristics of the pre-2015 cohort using the $\chi^{2}$ test, Student $t$ test, or the Fisher test. The risk of $\mathrm{DB}$ was estimated using sample proportions with 95\% confidence intervals (CIs), and the assessment of predictors of $\mathrm{DB}$ was performed using logistic regression models. We used the post-2015 cohort to validate the GSEED-RE model (GSEED-REa) and the original ACER model (ACERa). Model discrimination was compared by estimating the area under the receiver operating characteristic curve with 95\% CI and complemented with the estimation of the discrimination slope, which measured the mean 
difference in theoretical probabilities of DB between patients with and without observed DB (the higher and more significant the difference, the better). Calibration of the models was checked with the Hosmer-Lemeshow goodness-of-fit-test and complemented using the calibration plots of the predicted against the actual risk in deciles.

In addition to the 2 original models, 2 updated versions were assessed following the recommendations in the literature for risk prediction models. ${ }^{20}$ Therefore, we first tested the existing models and adjusted or updated these models when necessary using the post-2015 cohort. The first update recalibrated the original version of each model and named the models GSEED-REb and ACERb, respectively. The revised models consisted of adjustment of the intercept and other predictor regression coefficients by 1 overall adjustment factor (method 2 in Moons et $\mathrm{al}^{20}$ ), which is convenient when the outcome frequency differs between the development and test sample and/or the regression coefficients of the original model were overor underfitted. The second update re-estimated the models (GSEED-REc and ACERc) and maintained the variables of the original model but re-estimated the model parameters, which is convenient when the strength of certain predictors may be different in the test sample (method 3 in Moons et $\mathrm{al}^{20}$ ). Versions of the GSEED-RE model were validated using the total Spanish cohort (pre-2015 plus post2015 cohorts), and the ACER models were validated using the pre-2015 and the total Spanish cohort. Finally, a multivariate logistic regression was used to create a new model (GSEED-RE2) that included variables significant in the univariate logistic regression analysis for the post-2015 cohort and/or were included in the GSEED-RE and ACER models.

Anticoagulant and antiplatelet agents were included in 3 versions. Three possible options for the antiplatelet/anticoagulant variable in GSEED-RE2a were none, antiplatelet, and warfarin/acenocumarol/NOAC (dabigatran). Two possible options in GSEED-RE2b were no or yes. The summarized score system model in GSEED-RE2c was derived from GSEEDRE2b. The performance of all 9 models was assessed and compared using the post-2015 cohort. The pre-2015 cohort used in Albéniz et al, ${ }^{13}$ and the total Spanish cohort was used to validate GSEED-RE2a, GSEED-RE2b, and GSEEDRE2c. Statistical analysis was performed with SPSS version 20.0 (IBM Corp, Armonk, NY, USA) and R version 3.4.0 (R Foundation for Statistical Computing, Vienna, Austria).

\section{RESULTS}

\section{General characteristics of patients, lesions, and EMRs}

Overall, 1034 EMRs of large colorectal lesions were performed consecutively in 977 patients $(62.1 \%$ men; mean age, 68.1 years) (Table 1). The mean lesion size was $30.5 \mathrm{~mm}$ (SD, $10.5 \mathrm{~mm}$; range, 20-120). The ASA score was III or IV in 216 patients (24.3\%). One hundred sixty
(15.6\%) and 82 (8\%) patients were on antiplatelet and anticoagulant therapy, respectively (Table 1 ).

\section{DB rate and predictors}

DB occurred in 45 patients (4.5\%; 95\% CI, 3.3\%-6.0\%): in 17 patients (38\%) within 48 hours and in 18 patients (40\%) 3 to 7 days post-EMR. This DB rate of $4.5 \%$ was not significantly higher than the rate previously reported by our group ${ }^{13}$ (3.7\%; 95\% CI, 2.7\%-4.9\%).

Locations proximal to the splenic flexure (62.6\% vs $51.3 \%$, $P<.001)$ and proximal to the hepatic flexure $(51.0 \%$ vs $40.0 \%$, $P<.001)$ were significantly higher in patients in the post-2015 cohort than in the pre-2015 cohort. Splenic flexure and hepatic flexure were not included in their respective categories. The proportion of ASA score III or IV patients was lower for the post-2015 cohort (24.3\% vs 30.5\% in pre-2015 cohort, $P=.002)$ and the proportion of patients with antiplatelet prescriptions ( $16.4 \%$ vs $26.1 \%$, respectively). No other significant differences were found.

Univariate logistic regression analysis (Supplementary Table 1, available online at www.giejournal.org) identified the following variables as being significantly associated with DB: location proximal to the splenic flexure (OR, 2.84; 95\% CI, 1.31-6.16), location proximal to the hepatic flexure (OR, 1.96; 95\% CI, 1.04-3.69), antiplatelet use (OR, 2.51; 95\% CI, .99-6.34), warfarin/acenocumarol/ dabigatran use (OR, 4.54; 95\% CI, 2.14-9.63), and difficulty of the EMR (medium: OR, 1.52; 95\% CI, .653.51; high: OR, 3.23; 95\% CI, 1.41-7.40). Neither clipping nor other variables were significantly associated with DB.

\section{Performance assessment of the GSEED-RE and ACER models using the post-2015 cohort}

The specifications of models GSEED-REa, GSEED-REb, and GSEED-REc and models ACERa, ACERb, and ACERc are given in Supplementary Table 2 (available online at www.giejournal.org). The scores corresponding to each independent variable according to each predictive model are shown in Table 2.

The GSEED-RE model was applied to the post-2015 cohort. GSEED-REa resulted in an area under the curve (AUC) of .62 (95\% CI, .52-.71), a discrimination slope of $.019(P=.036)$, and a significant Hosmer-Lemeshow test. These results suggest low discrimination ability and poor calibration (first column of Table 3), which was corroborated in the calibration plot (upper row, first graph, Supplementary Fig. 1, available online at www. giejournal.org). The recalibrated version of the GSEEDREb model produced the same receiver operating characteristic curve and better calibration results (Hosmer-Lemeshow $P=.376$ and good calibration plot), and the re-estimated version, GSEED-REc, yielded a slightly better AUC (.64; 95\% CI, .54-.74) and better calibration (Hosmer-Lemeshow $P=.215$ ) but not an optimum calibration plot. Therefore, none of the models reached the minimum AUC of .70, despite good calibration in GSEED-REb and GSEED-REc. 


\begin{tabular}{|c|c|c|c|c|}
\hline \multirow[t]{2}{*}{ Age group } & $<75$ y & 0 & - & - \\
\hline & $>75 y$ & 1 & - & - \\
\hline \multirow[t]{2}{*}{ ASA (or comorbidity) $\dagger$} & I-II (or none) & 0 & 0 & 0 \\
\hline & III-IV ( $\geq 1$ comorbidity) & 1 & 1 & 1 \\
\hline \multirow{3}{*}{ Size } & $\geq 30$ & - & 2 & - \\
\hline & $<40$ & 0 & - & 0 \\
\hline & $\geq 40$ & 1 & - & 2 \\
\hline Aspirin use & No & 0 & - & - \\
\hline \multirow[t]{2}{*}{ Proximal to the hepatic flexure } & No & 0 & 0 & 0 \\
\hline & Yes & 3 & 2 & 3 \\
\hline \multirow[t]{2}{*}{ Epinephrine } & Yes & - & 0 & - \\
\hline & No & - & 1 & - \\
\hline \multirow[t]{2}{*}{ Antiplatelet/anticoagulant } & No & - & - & 0 \\
\hline & Yes & - & - & 3 \\
\hline
\end{tabular}

GSEED-RE, Endoscopic Resection Group of the Spanish Society of Endoscopy; ACER, Australian Colonic Endoscopic Resection; ASA, American Society of Anesthesiologists, GSEED$R E 2$, our updated model.

*The total score for each model is the sum of the scores of each variable.

$\dagger$ American Society of Anesthesiologists score was used for the GSEED-RE model, and comorbidity (none or at least 1) was used for ACER and GSEED-RE2 models.

The percentages of patients with DB in the low-, medium-, and high-risk categories were 3.5\%, 5.9\%, and 10.5\%, respectively (Table 4), which suggests a consistent gradient, although it was less marked than the pre-2015 cohort $\left(.6 \%, 5.5 \%\right.$, and $40 \%$, respectively). ${ }^{13}$

The ACER model ACERa resulted in an AUC of .61 (95\% CI, .52-.71), a discrimination slope of .027 $(P=.014)$, and a significant Hosmer-Lemeshow test. These results suggest low discrimination ability and poor calibration (first column of Table 3), which was corroborated in the calibration plot (upper row, middle graph in Supplementary Fig. 1). The recalibrated version of the model, ACERb, produced a similar receiver operating characteristic curve and better calibration results (Hosmer-Lemeshow $P=.515$ and a better calibration plot), and the re-estimated version, ACERc, yielded a slightly better AUC (.65; 95\% CI, .57-.73), better calibration (Hosmer-Lemeshow $P=.680$ ), and acceptable calibration plots. Therefore, similar to the GSEED-RE-based models, none of the ACER-based models reached the minimum AUC of .70, although calibration was sufficient in ACERb and ACERc. The percentages of patients with DB in the low-, medium-, and high-risk categories were $3.3 \%, 4.0 \%$, and $8.9 \%$, respectively (Table 4), which suggests a consistent gradient, similar to the pre-2015 cohort. ${ }^{13}$

The covariates proposed in the GSEED-RE2a included comorbidity, size $\geq 40 \mathrm{~mm}$, location proximal to the hepatic flexure, and antiplatelet/anticoagulant use in 3 categories (score from 0 to 9 points; Table 2). The resulting AUC was
.69 (95\% CI, .60-.77) with a discrimination slope of .037 $(P<.001)$. This model showed a reasonable discrimination ability and a nonsignificant Hosmer-Lemeshow test with an acceptable calibration plot (Table 3 and upper row, third graph of Supplementary Fig. 1). The results obtained with GSEED-RE2b presented similar AUC, discrimination slope, and calibration (Table 3). GSEED-RE2c is a simplified version of GSEED-RE2b. This model yielded an AUC of .69 (95\% CI, .59-.78) and a discrimination slope of .033 $(P<.001)$. The Hosmer-Lemeshow test did not show good calibration $(P=.037)$, although the calibration plots were acceptable (upper row, third graph of Supplementary Fig. 1). Therefore, GSEED-RE2c provided slightly better discrimination results than the GSEED-RE and ACER models, with an AUC curve close to .7, a discrimination slope that doubled in magnitude, and reasonable calibration plots. The percentages of patients with DB in the low-, medium-, and high-risk categories were $2.2 \%, 4.9 \%$, and $17.1 \%$, respectively, which suggests a consistent gradient that was more marked than the GSEED-RE and ACER models.

\section{Validation of the new model applied to the pre-2015 and total Spanish cohorts}

Model performance was assessed using the pre-2015 cohort and showed good results for GSEED-RE2 in all 3 versions; for example, the simplified GSEED-RE2c provided an AUC of .73 (95\% CI, .66-.80), a discrimination 


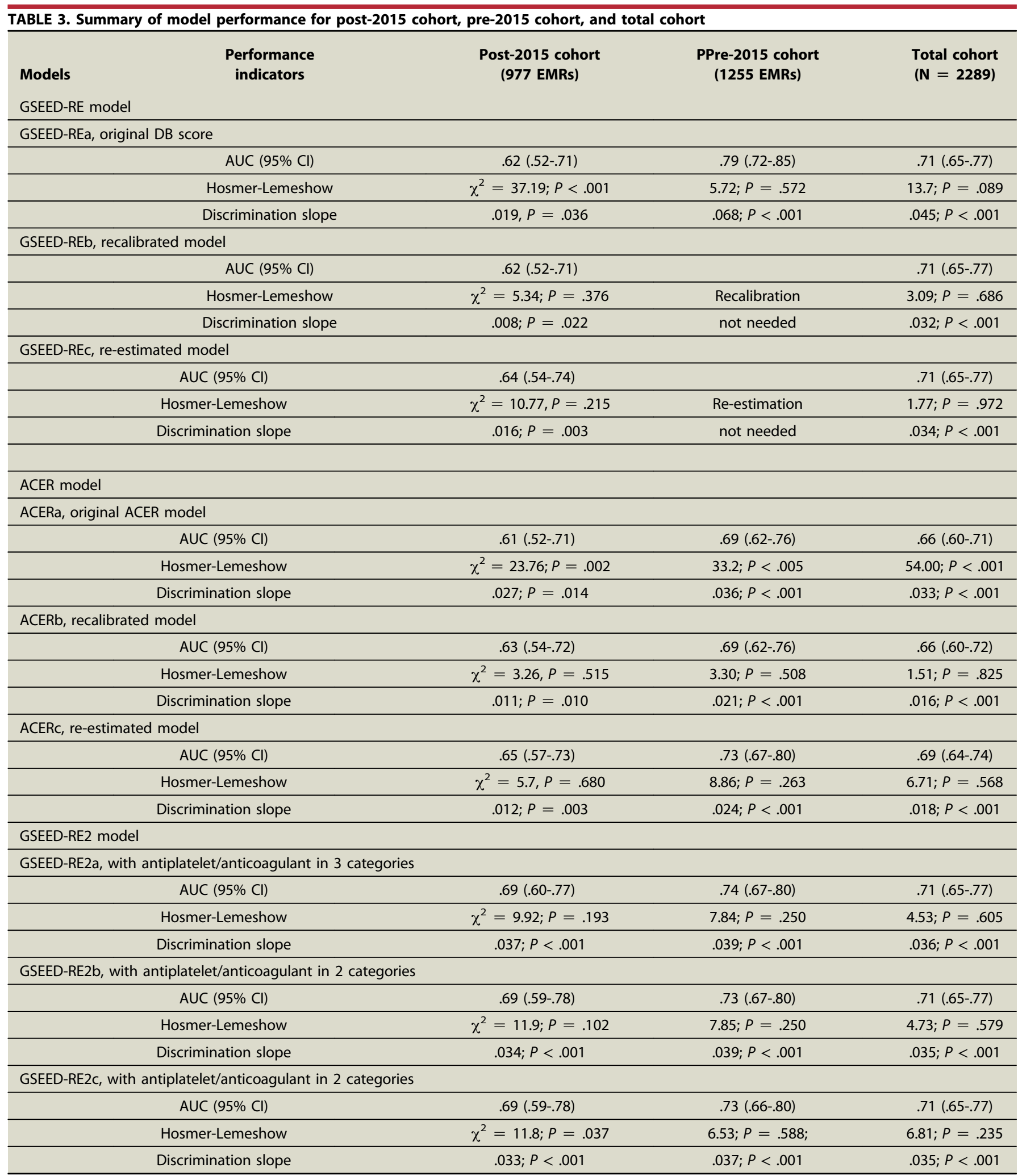

$A C E R$, Australian Colonic Endoscopic Resection; Cl, confidence interval; $A U C$, area under the curve; DB, delayed bleeding; GSEED-RE, Endoscopic Resection Group of the Spanish Society of Endoscopy.

slope of $.037(P<.001)$, and acceptable calibration results (see Hosmer-Lemeshow $P=.588$ in Table 3 and middle row of Supplementary Fig. 1).
DB occurred in 91 patients in the total Spanish cohort (pre-2015 plus post-2015 cohorts, 2289 EMRs) (OR, 4.0; 95\% CI, 3.3\%-4.9\%). The performance of the models in 


\begin{tabular}{|c|c|c|c|c|c|c|}
\hline & \multicolumn{2}{|c|}{ GSEED-RE (score 0-10) } & \multicolumn{2}{|c|}{ ACER (score 0-6) } & \multicolumn{2}{|c|}{ GSEED-RE2 (score 0-9) } \\
\hline \multicolumn{7}{|l|}{ Post-2015 cohort } \\
\hline Low risk & $0-3$ & 3.5 & $0-1$ & 3.3 & $0-3$ & 2.2 \\
\hline \multicolumn{7}{|l|}{ Pre-2015 cohort } \\
\hline Low risk & $0-3$ & .6 & $0-1$ & .4 & $0-3$ & 2.1 \\
\hline Medium risk & 4-7 & 5.5 & $2-4$ & 3.6 & $4-6$ & 3.4 \\
\hline High risk & $8-10$ & 40 & $5-6$ & 10.0 & $7-9$ & 13.4 \\
\hline \multicolumn{7}{|l|}{ Total cohort } \\
\hline High risk & $8-10$ & 27.3 & $5-6$ & 9.8 & $7-9$ & 14.8 \\
\hline
\end{tabular}

ACER, Australian Colonic Endoscopic Resection; DB, delayed bleeding; GSEED-RE, Endoscopic Resection Group of the Spanish Society of Endoscopy.

the total cohort was acceptable, with all GSEED-RE2 versions exhibiting an AUC over .70 in all cases (Table 3). A summary diagram derived from the total cohort is shown in Figure 1, which indicates the weights assigned to each variable and the risk associated with each score value.

\section{DISCUSSION}

DB is the most common serious adverse event after EMR. It is associated with a non-negligible morbidity rate and resource use. However, no guidelines suggest strategies to prevent $\mathrm{DB}$, and the risk factors associated with DB described in observational studies are controversial..$^{2-4}$

Our multicenter prospective study validated our previous model and performed the first external validation of the ACER model by another group. We also developed a new model that predicted DB with reasonable accuracy. This model includes 4 easily identifiable variables that can be assessed before performing any procedure: major comorbidity, size greater than $4 \mathrm{~cm}$, antiplatelet or anticoagulant use, and lesions located proximal to the hepatic flexure.

We assessed the performance of the GSEED-RE and the ACER models in their original versions using 3 large prospective cohorts (pre-2015, post-2015, and total cohort). Overall, the performance of the original GSEED-RE and ACER models was fair. However, the recalibrated and reestimated models achieved better performance, but it was still suboptimal. These results suggest that the previous models needed to be refined before recommended use in clinical practice. This result motivated us to develop other models that may offer improvements, although the performance did not increase greatly compared with previous models.
First, unlike the GSEED-RE or the ACER models, the new model provided acceptable AUCs across cohorts (AUC .69-.74), which suggests higher stability compared with previous proposals and lower susceptibility to changes in datasets. Calibration plots were also more stable across cohorts and model versions (original score, recalibrated, and refitted) for the new model. We hypothesized that variations in the pre-2015 cohort and post-2015 cohort using the GSEED-RE model reflected an overfit of the regression coefficients because of the instability produced for having a very small probability of events, which could generate more optimistic results. The reasons were different for the ACER model because the setting in which the model was developed had higher DB risks than in our study (6.7\% vs $4.5 \%$ in our setting), which undermined the calibration and estimation results. Second, the discrimination slope for the new model (Table 3) was higher (>.03) and more consistent across cohorts than the GSEED-RE and ACER proposals, which suggests a higher discriminating ability and consistency in predicting DB. Third, similarly to the ACER model, the probability of DB in the highrisk category was stable across cohorts and was higher than the ACER model regardless of the cohort $13.4 \%$ and $17.1 \%$ for the new model vs $10.0 \%$ and $8.9 \%$ for the ACER model, in the pre-2015 and post-2015 cohorts, respectively), which suggests better detection of highrisk patients. Conversely, the probabilities of DB in the high-risk group using the GSEED-RE model varied widely between cohorts. Fourth, the new score is easy to calculate because it only involves 4 variables that are easily recognizable by an endoscopist and/or the clinician and that are known before the procedure is scheduled. The GSEED-RE model requires information on prevention measures (clip closure of the mucosa 


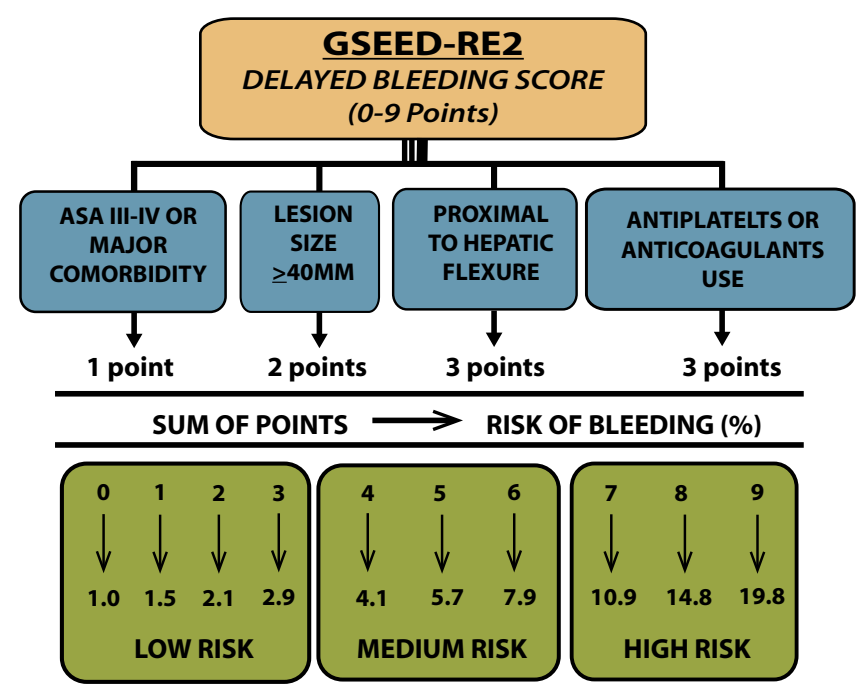

Figure 1. New delayed bleeding risk score for colorectal EMR. $D B$, Delayed bleeding; ASA, American Society of Anesthesiologists; GSEED-RE2, our updated model.

defect) and includes more variables. Notably, the new model eliminated certain variables that were not consistently significant in different cohorts (clip closure, age, and adrenaline use in the injection solution) and preserved variables of other models, such as major comorbidity ( 1 point), size $\geq 40$ ( 2 points), and location proximal to the hepatic flexure (3 points), but it also included a new meaningful variable, antiplatelet/anticoagulant use ( 3 points). The first 3 variables are nonmodifiable, and the last variable mentioned may be modified in some patients, depending on their risk of thrombosis. In the new model, the use of preventive methods such as clip closure during EMR was not included in the score because the main objective of this score was to design a tool that helps the clinician classify patients by their risk of DB beforehand and design, "a priori," the best prophylactic strategy according to it.

The trichotomous categorization of the variable antiplatelet/anticoagulant use (no/antiplatelet/anticoagulants) yielded better calibration results than previous models (AUC .69), but it is more convenient to categorize it as a dichotomous variable (no or yes) because of the low number of DB cases and the low proportion of antiplatelet/anticoagulant use in the sample (GSEED-RE2c). Therefore, GSEED-RE2c exhibited the best properties.

The model proposed offers a series of significant advantages. First, this model is a useful informative tool that predicts DB risk in 10 groups (scores 0-9) placed in 3 categories (Fig. 1), which may allow taking preventive measures for each patient based on his or her score. One of the benefits of this model lies in the identification of patients within the high-risk category before EMR is performed. Furthermore, the use of this model makes it possible to identify patients who are especially prone to DB (score 9) because 1 of 5 patients (20\%) in this group will actually bleed. Second, only $10 \%$ of patients $(n=209)$ in our study were in the high-risk category. Because clinical resources are limited, this model allows a better selection of patients who may benefit most from preventive measures. Previous evidence did not support the closing of all mucosal defects, but 2 welldesigned randomized controlled trials support their use in large nonpedunculated polyps located in the proximal colon $^{21}$ and in patients with medium or high risk of $\mathrm{DB}^{22}$ Therefore, this measure could be cost-effective for the high-risk category. Clipping and complete closure of the mucosal defects were not associated with a DB in the present study, likely because it was not designed for this aim.

Third, a practical implication of this score is that a tailored anticoagulation management may be performed depending on the risk of developing DB (eg, later resumption for patients with scores between 7 and 9). Fourth, the score may also be useful for the management of logistics, such as the need of family support or to stay close to the hospital at least during the first week for patients in the high-risk category. Finally, the new model may improve the selection of candidates for inclusion in clinical trials, which ensures data that are more reliable and reduces sample sizes. The previously discussed advantages may help optimize the available resources and contribute to cost reduction.

The main strengths of the present study are the large number of patients and lesions included and participation of multiple endoscopists and institutions. Limitations are associated with the multicenter and observational nature of the study, such as the possible selection bias because of under-reported cases. However, all participating endoscopists included at least 20 patients, and their involvement was stable during the study period. The resection methods also varied considerably between endoscopy units, as does the probability of clipping, which also varies among hospitals and endoscopists. Nevertheless, our findings reflect daily clinical practice.

Second, a score component is the presence of a major comorbidity. This variable is heterogeneous but is likely a surrogate marker of greater susceptibility to adverse events. We hypothesized that the low number of ASA score II plus IV patients compared with the rest of the sample undermined the impact of this variable. Third, we did not include in the derived model the use of prophylactic measures, such as the use of clips, as covariates because we wanted to produce a tool to identify DB risk before any procedure was performed, and to do so only patient and lesion characteristics could be included. In addition, clipping was not carried out in a protocolized fashion in this study. When the study was designed, there was little evidence about the effect of clipping as well as factors associated with preventing DB. Therefore, 
a uniform recommendation for clipping was not made at the beginning of the study, and clipping was left at the discretion of the endoscopist. We are aware that this could have affected our results, because clipping could be related to clinical and lesion variables and DB, but we considered that the effect of clipping on DB had to be better assessed with a clinical study specifically designed with that aim. ${ }^{22}$ Finally, the probability of bleeding for the high-risk group using the new model seems low (14.8\%), but it was 7 times higher than the low-risk group (2.2\%) and 3 times higher than the medium-risk group (4.1\%). Therefore, in relative terms, it provides very different magnitudes of risk depending on the scoring result.

In conclusion, the validation of the GSEED-RE and ACER models yielded suboptimal results. However, the updated GSEED-RE and ACER models achieved acceptable prediction levels for DB. The new model showed good calibration and discrimination properties and may assist endoscopists in predicting DB after EMR. This DB predicting tool may help guide prophylactic treatments and periprocedural care. Furthermore, it may be a useful tool in clinical trials to evaluate prevention strategies after large EMRs. Other working groups should validate this model and particularly test the impact of the number of antiplatelet and anticoagulant categories on model performance.

\section{ACKNOWLEDGMENTS}

We thank the GSEED-RE for the continuous, anonymous, and tireless collaboration within this working group and the Navarrabiomed-Miguel Servet Foundation for their support with the statistical analyses. Research support for this study was received from "La Caixa/Caja Navarra" Foundation (ID 100010434; project PR15/11100006). English editing and manuscript formatting were performed by American Journal Experts, LLC (www.aje.com).

\section{REFERENCES}

1. Othman MO, Wallace MB. Endoscopic mucosal resection (EMR) and endoscopic submucosal dissection (ESD) in 2011, a Western perspective. Clin Res Hepatol Gastroenterol 2011;35:288-94.

2. Burgess NG, Metz AJ, Williams SJ, et al. Risk factors for intraprocedural and clinically significant delayed bleeding after wide-field endoscopic mucosal resection of large colonic lesions. Clin Gastroenterol Hepatol 2014;12:651-61.

3. Hassan C, Repici A, Sharma P, et al. Efficacy and safety of endoscopic resection of large colorectal polyps: a systematic review and metaanalysis. Gut 2015;65:806-20.

4. Metz AJ, Bourke MJ, Moss A, et al. Factors that predict bleeding following endoscopic mucosal resection of large colonic lesions. Endoscopy 2011;43:506-11.

5. Sorbi D, Norton I, Conio M, et al. Postpolypectomy lower Gl bleeding: descriptive analysis. Gastrointest Endosc 2000;51:690-6.
6. Hui AJ, Wong RM, Ching JY, et al. Risk of colonoscopic polypectomy bleeding with anticoagulants and antiplatelet agents: analysis of 1657 cases. Gastrointest Endosc 2004;59:44-8.

7. Sawhney MS, Salfiti N, Nelson DB, et al. Risk factors for severe delayed postpolypectomy bleeding. Endoscopy 2008;40:115-9.

8. Shiffman ML, Farrel MT, Yee YS. Risk of bleeding after endoscopic biopsy or polypectomy in patients taking aspirin or other NSAIDS. Gastrointest Endosc 1994;40:458-62.

9. Manocha $D$, Singh $M$, Mehta $N$, et al. Bleeding risk after invasive procedures in aspirin/NSAID users: polypectomy study in veterans. Am J Med 2012;125:1222-7.

10. Pan A, Schlup M, Lubcke $R$, et al. The role of aspirin in postpolypectomy bleeding-a retrospective survey. BMC Gastroentero 2012;12:138

11. Watabe H, Yamaji Y, Okamoto M, et al. Risk assessment for delayed hemorrhagic complication of colonic polypectomy: polyp-related factors and patient-related factors. Gastrointest Endosc 2006;64 73-8.

12. Kim HS, Kim TI, Kim WH, et al. Risk factors for immediate postpolypectomy bleeding of the colon: a multicenter study. Am J Gastroentero 2006;101:1333-41.

13. Albéniz $E$, Fraile $M$, Ibañez $B$, et al. A scoring system to determine risk of delayed bleeding after endoscopic mucosal resection of large colorectal lesions. Clin Gastroenterol Hepatol 2016;14:1140-7.

14. Bahin FF, Rasouli KN, Byth K, et al. Prediction of clinically significant bleeding following wide-field endoscopic resection of large sessile and laterally spreading colorectal lesions: a clinical risk score. Am Gastroenterol 2016;111:1115-22.

15. Charlson ME, Pompei $\mathrm{P}$, Ales $\mathrm{KL}$, et al. A new method of classifying prognostic comorbidity in longitudinal studies: development and validation. J Chronic Dis 1987;40:373-83.

16. Albéniz E, Fraile M, Guarner G, et al. Clinical usefulness of the SMSA difficulty score and comparison with a subjective score for the management of large non-pedunculated colorectal lesions. A multicenter study from the Spanish Endoscopy Society Endoscopic Resection Group. Un Eur Gastroenterol J 2017;5(5 Suppl): A446.

17. Bourke M. Endoscopic mucosal resection in the colon: a practical guide. Techn Gastrointest Endosc 2011;13:35-49.

18. Sakamoto N, Beppu K, Matsumoto K, et al. "Loop Clip", a new closure device for large mucosal defects after EMR and ESD. Endoscopy 2008;40(Suppl 2):E97-8.

19. Veitch AM, Vanbiervliet G, Gershlick AH, et al. Endoscopy in patients on antiplatelet or anticoagulant therapy, including direct oral anticoagulants: British Society of Gastroenterology (BSG) and European Society of Gastrointestinal Endoscopy (ESGE) guidelines. Endoscopy 2016;48: 385-402.

20. Moons KG, Kengne AP, Grobbee DE, et al. Risk prediction models. II. External validation, model updating, and impact assessment. Heart 2012;98:691-698.21.

21. Pohl H, Grimm IS, Moyer MT, et al. Clip closure prevents bleeding after endoscopic resection of large colon polyps in a randomized trial. Gastroenterology 2019;157:977-84.

22. Albéniz E, Álvarez-González MA, Espinós JC, et al. Clip closure after resection of large colorectal lesions with substantial risk of bleeding. Gastroenterology 2019;157:1213-21.

Abbreviations: ACER, Australian Colonic Endoscopic Resection; ASA, American Society of Anesthesiologists; $A U C$, area under the curve; DB, delayed bleeding; GSEED-RE, Endoscopic Resection Group of the Spanish Society of Endoscopy; OR, odds ratio.

DISCLOSURE: The following author disclosed financial relationships relevant to this publication: E. Redondo-Cerezo: Advisory board for Cook Endoscopy. All other authors disclosed no financial relationships relevant to this publication. 
See CME section; p. 943.

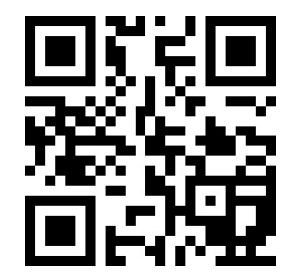

Use your mobile device to scan this QR code and watch the author interview. Download a free $Q R$ code scanner by searching "QR Scanner" in your mobile device's app store.

Copyright $\odot 2020$ by the American Society for Gastrointestinal Endoscopy 0016-5107/\$36.00

https://doi.org/10.1016/j.gie.2019.10.013

Received April 26, 2019. Accepted October 3, 2019.

Current affiliations: Complejo Hospitalario de Navarra, Pamplona, Spain (1), Hospital Universitario de Canarias, Santa Cruz de Tenerife, Spain (2), Hospital San Pedro, Logroño, Spain (3), Navarrabiomed-Complejo Hospitalario de Navarra-UPNA and REDISSEC, Pamplona, Spain (4), Hospital de la Santa Creu y Sant Pau, Universitat Autónoma de Barcelona, Barcelona, Spain (5), Hospital Juan Canalejo, La Coruña, Spain (6), Hospital del Mar, Barcelona, Spain (7), Hospital Clínico Universitario Lozano Blesa, Zaragoza, Spain (8), Hospital Clinic, Barcelona, Spain (9), Hospital Universitario HM Montepríncipe, Madrid, Spain (10), Hospital Universitario Puerta de Hierro, Madrid, Spain (11), Hospital General
Universitario Gregorio Marañón, Madrid, Spain (12), Complejo Hospitalario de Vigo, Vigo, Spain (13), Hospital Universitario Donostia, Donostia-San Sebastián, Spain (14), Hospital de Valdecilla, Santander, Spain (15), Hospital Universitario Mútua Terrassa, Spain (16), Hospital de Galdakao, Usansolo, Galdakao, Spain (17), Complejo Asistencial Universitario de Salamanca, IBSAL, Salamanca, Spain (18), Complejo Hospital Virgen de la Salud, Toledo, Spain (19), Hospital Universitario Virgen de las Nieves, Granada, Spain (20), Clínica ServiDigest, Barcelona, Spain (21), Consorcio Hospitalario Provincial Castellón, Castellón, Spain (22), Hospital General de Catalunya, Barcelona, Spain (23), Complejo Hospitalario de Ourense, Ourense, Spain (24), Hospital General de Ciudad Real, Ciudad Real, Spain (25), Hospital Universitario Lucus Augusti, Lugo, Spain (26), Hospital Universitario Virgen Macarena, Sevilla, Spain (27), Hospital Moisès Broggi, Sant Joan Despí, Spain (28), Centro Andaluz de Gastroenterología Integral, Sevilla, Spain (29), Complejo Hospitalario La Mancha Centro, Alcázar de San Juan, Ciudad Real, Spain (30), Hospital Universitario La Paz, Madrid, Spain (31), Navarrabiomed/ UPNA /IdisNA, Pamplona, Spain (32).

Reprint requests: Eduardo Albéniz, MD, Gastroenterology Department, Endoscopy Unit, Complejo Hospitalario de Navarra, Navarrabiomed Research Institute/ Public University of Navarra/IdiSNA, Irunlarrea 3, 31008, Pamplona, Navarra, Spain.

If you would like to chat with an author of this article, you may contact Dr Albéniz at edualbeniz@hotmail.com.

\section{GIE on Facebook}

Follow GIE on Facebook to receive the latest news, updates, and links to author interviews, podcasts, articles, and tables of contents. Search on Facebook for "GIE: Gastrointestinal Endoscopy" or use this QR code for quick access to our recent posts.

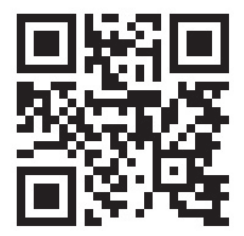




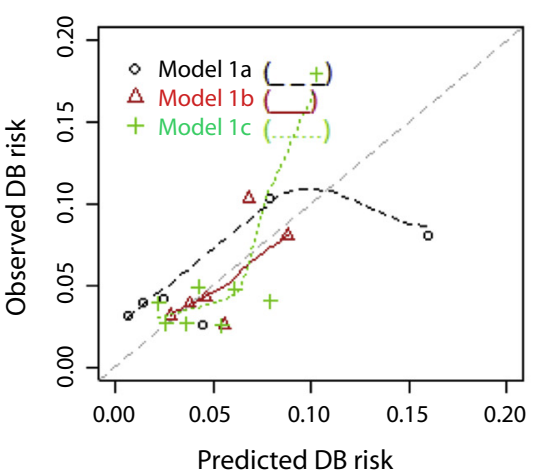

VALIDATION COHORT (POST-2015)
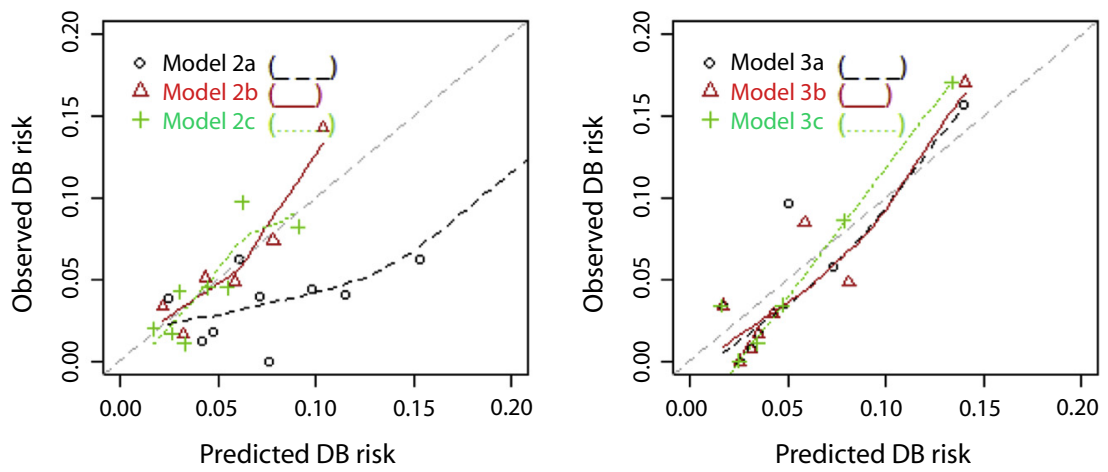

DERIVATION COHORT (PRE-2015)
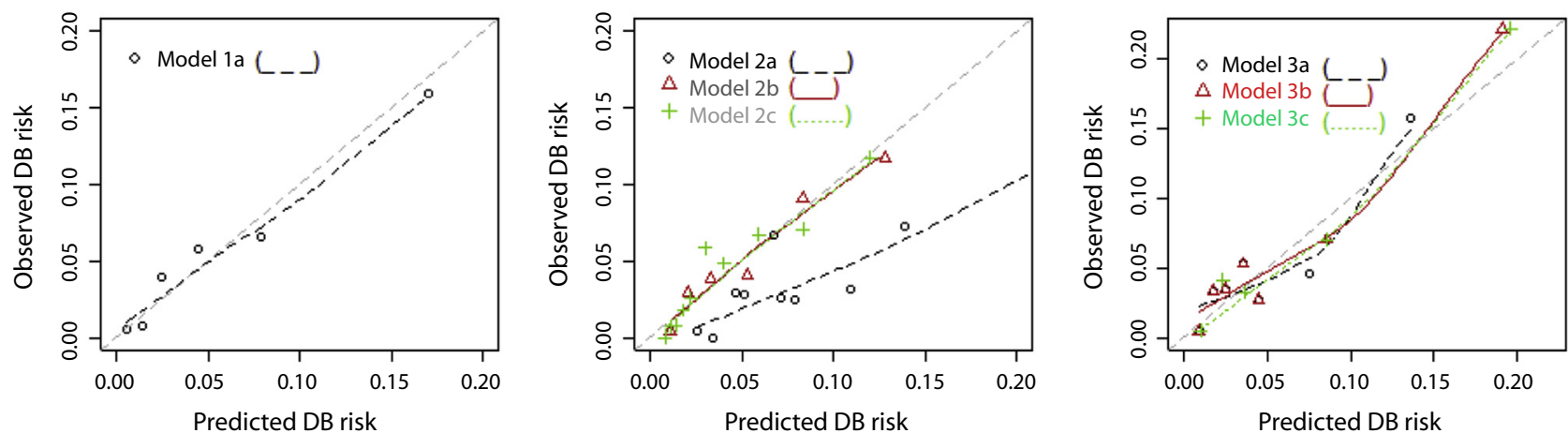

TOTAL COHORT
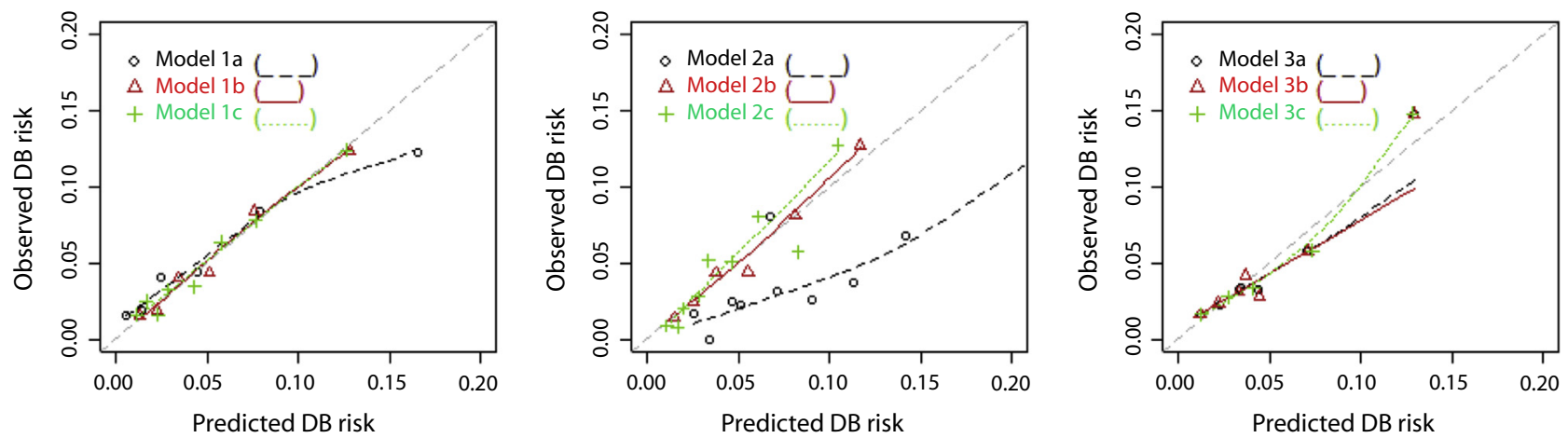

Supplementary Figure 1. Calibration plots for the GSEED-RE (Model 1), ACER (Model 2), and GSEED-RE2 (Model 3). A, validated models; B, re-calibrated models; $\mathbf{C}$, re-estimated models. 


\begin{tabular}{|c|c|c|c|}
\hline Variable & Categories & Odds ratio $(95 \% \mathrm{CI})$ & $P$ value \\
\hline \multirow[t]{2}{*}{ Sex } & Female & Reference & \\
\hline & Male & $.91(.49-1.67)$ & .755 \\
\hline \multirow[t]{2}{*}{ Age } & $<75 \mathrm{y}$ & Reference & \\
\hline & $\geq 75$ y & $1.26(.67-2.37)$ & .480 \\
\hline \multirow[t]{2}{*}{ ASA score } & $|-I|$ & Reference & \\
\hline & III-IV & $1.65(.85-3.21)$ & .139 \\
\hline \multirow[t]{2}{*}{ Comorbidity } & No & Reference & \\
\hline & Yes & $2.11(.99-4.47)$ & .053 \\
\hline \multirow[t]{3}{*}{ Antiplatelet/ anticoagulants } & No & Reference & \\
\hline & Antiplatelet/dual antiplatelet & $2.51(.99-6.34)$ & $<.001$ \\
\hline & Warfarin/acenocumarol/dabigatran & $4.54(2.14-9.63)$ & \\
\hline \multirow[t]{2}{*}{ Paris } & 0 (flat) & Reference & .809 \\
\hline & 1 (protruded) & $.93(.50-1.72)$ & \\
\hline \multirow[t]{2}{*}{ Size } & $<30 \mathrm{~mm}$ & Reference & .154 \\
\hline & $\geq 30 \mathrm{~mm}$ & $1.56(.85-2.86)$ & \\
\hline \multirow[t]{2}{*}{ Size } & $<40 \mathrm{~mm}$ & Reference & .210 \\
\hline & $\geq 40 \mathrm{~mm}$ & $1.51(.79-2.90)$ & \\
\hline \multirow[t]{2}{*}{ Proximal to the splenic flexure (splenic flexure not included) } & No & Reference & \\
\hline & Yes & $2.84(1.31-6.16)$ & .008 \\
\hline \multirow[t]{2}{*}{ Proximal to the hepatic flexure (hepatic flexure not included) } & No & Reference & \\
\hline & Yes & 1.96 (1.04-3.69) & .037 \\
\hline \multirow[t]{2}{*}{ Histology } & Serrated & Reference & \\
\hline & Adenoma & $2.15(.66-7.03)$ & .207 \\
\hline \multirow[t]{2}{*}{ Histology } & Benign & Reference & \\
\hline & Malignant & $1.14(.61-2.12)$ & .675 \\
\hline \multirow[t]{3}{*}{ Difficult EMR } & Low & Reference & \\
\hline & Medium & $1.52(.65-3.51)$ & .013 \\
\hline & High & $3.23(1.41-7.40)$ & \\
\hline \multirow[t]{2}{*}{ Resection } & Piecemeal & Reference & \\
\hline & En bloc & $.54(.23-1.29)$ & .167 \\
\hline \multirow[t]{2}{*}{ Adrenaline } & No & Reference & \\
\hline & Yes & $.88(.47-1.65)$ & .693 \\
\hline \multirow[t]{2}{*}{ Argon plasma } & No & Reference & \\
\hline & Yes & 0 (none of 44 ) & NA \\
\hline \multirow[t]{3}{*}{ Clipping } & No (0) & Reference & .945 \\
\hline & Punctual (1) & $1.14(.52-2.48)$ & \\
\hline & Complete (2-4) & $1.07(.52-2.21)$ & \\
\hline Complete closure with hemoclips & Yes & Reference & .925 \\
\hline \multirow[t]{3}{*}{ Electrocautery } & Blended & Reference & .511 \\
\hline & Cut current & 0 (none of 2 ) & \\
\hline & Coagulation current & $1.60(.69-3.68)$ & \\
\hline
\end{tabular}

$A S A$, American Society of Anesthesiologists; $C l$, confidence interval; $N A$, not applicable. 


\section{SUPPLEMENTARY TABLE 2. Multivariate logistic regression model specification for the GSEED-RE, ACER, and GSEED-RE2 models in the total} cohort*

\begin{tabular}{|c|c|c|c|c|}
\hline & Models & $f(x)$ with the post-2015 & $f(x)$ with the pre-2015 & $f(x)$ with the total cohort \\
\hline \multicolumn{5}{|c|}{ GSEED-RE model } \\
\hline a: & Original & $-6.06+0.60 \times$ score $\dagger$ & $-6.06+0.60 \times$ score $\dagger$ & $-6.06+0.60 \times$ score $\dagger$ \\
\hline b: & Recalibrated & $-3.86+0.21 \times$ score $\dagger$ & Not needed & $-4.99+0.42 \times$ score $\dagger$ \\
\hline c: & Re-estimated & $\begin{array}{c}-3.70-0.28 \times \text { age }_{\{>75\}}+ \\
0.45 \times \mathrm{ASA}_{\{>11\}}+0.53 \times \text { size }_{\{>40\}}+ \\
0.22 \times \text { aspirin }_{\{\text {not removed\} }}- \\
\left.0.04 \times \text { closure }_{\{\text {not complete }}\right\}+ \\
0.92 \times \text { proximal }_{\{\text {transverse }}\end{array}$ & Not needed & $\begin{array}{c}-4.85+0.35 \times \text { age }_{\{>75\}}+0.55 \times \mathrm{ASA}_{\{>11\}}+ \\
0.62 \times \text { size }_{\{>40\}}+0.78 \times \text { aspirin }_{\{\text {not removed }\}}- \\
\left.0.55 \times \text { closure }_{\{\text {not complete }}\right\}+ \\
1.27 \times \text { proximal }_{\{\text {transverse }}\end{array}$ \\
\hline \multicolumn{5}{|c|}{ ACER model } \\
\hline a: & Original & $\begin{array}{c}-3.34+0.92 \times \text { size }_{\{>30\}}+0.85 \times \\
\text { proximal } \\
\text { \{colon\} } \\
0.43 \times \text { comorbidity }^{-} \\
0.57 \times \text { epinephrine }\end{array}$ & $\begin{array}{c}-3.34+0.92 \times \text { size }_{\{>30\}}+ \\
0.85 \times \text { proximal }_{\{\text {colon\} }}+ \\
0.43 \times \text { comorbidity }- \\
0.57 \times \text { epinephrine }\end{array}$ & $\begin{array}{c}-3.34+0.92 \times \operatorname{size}_{\{>30\}}+0.85 \times \\
\text { proximal } \\
\text { colon\} } \\
\text { comorbidity }-0.57 \times \text { epinephrine }\end{array}$ \\
\hline \multicolumn{5}{|c|}{ GSEED-RE2 model } \\
\hline a: & + antiplatelet 3 cat & $\begin{array}{c}-4.21+0.57 \times \text { size }_{\{>40\}}+ \\
0.78 \times \text { proximal }_{\{\text {transverse\} }}+ \\
0.32 \times \text { comorbidity }+ \\
0.88 \times \text { antiplatelet } \\
1.37 \times \text { antiplatelet } / \mathrm{AC}\end{array}$ & $\begin{array}{c}-4.82+0.69 \times \text { size }_{\{>40\}}+ \\
1.52 \times \text { proximal }_{\{\text {transverse\} }}+ \\
0.25 \times \text { comorbidity }+ \\
0.98 \times \text { antiplatelet }_{\{y e s\}}+ \\
0.84 \times \text { antiplatelet } / A C\end{array}$ & $\begin{array}{c}-4.55+0.63 \times \text { size }_{\{>40\}}+ \\
1.19 \times \text { proximal }_{\{\text {transverse }}+ \\
0.29 \times \text { comorbidity }+ \\
0.92 \times \text { antiplatelet }_{\{y e s\}}+ \\
1.12 \times \text { antiplatelet/AC }\end{array}$ \\
\hline b: & + antiplatelet 2 cat & $\begin{array}{c}-4.23+0.60 \times \text { size } \dagger+ \\
0.79 \times \text { proximal }_{\text {\{transverse }}+ \\
0.33 \times \text { comorbidity }^{+} \\
1.17 \times \text { antiplatelet }_{\text {\{yes\} }}\end{array}$ & $\begin{array}{c}-4.82+0.69 \times \text { size }+ \\
1.51 \times \text { proximal }_{\text {transverse\} }}+ \\
0.24 \times \text { comorbidity }^{+} \\
0.93 \times \text { antiplatelet }_{\{y e s\}}\end{array}$ & $\begin{array}{c}-4.56+0.63 \times \text { size }_{\{>40\}}+ \\
1.20 \times \text { proximal }_{\{\text {transverse\} }}+ \\
0.31 \times \text { comorbidity }^{+} \\
1.00 \times \text { antiplatelet }_{\{\text {yes }}\end{array}$ \\
\hline
\end{tabular}

ACER, Australian Colonic Endoscopic Resection; ASA, American Society of Anesthesiologists; GSEED-RE, Endoscopic Resection Group of the Spanish Society of Endoscopy; $A C$, Anticoagulants.

*Specification of model parameters: $\log (P /(1-P))=f(x)$, where $P$ is probability of delayed bleeding

$\dagger$ †Scores as defined in Table 3. 


\title{
Clip Closure After Resection of Large Colorectal Lesions With Substantial Risk of Bleeding
}

\author{
Eduardo Albéniz, ${ }^{1}$ Marco Antonio Álvarez, ${ }^{2}$ Jorge C. Espinós, ${ }^{3}$ Oscar Nogales, ${ }^{4}$
}

Carlos Guarner, ${ }^{5}$ Pedro Alonso, ${ }^{6}$ Manuel Rodríguez-Téllez, ${ }^{7}$ Alberto Herreros de Tejada, ${ }^{8}$ José Santiago, ${ }^{8}$ Marco Bustamante-Balén, ${ }^{9}$ Joaquín Rodríguez Sánchez, ${ }^{10}$ Felipe Ramos-Zabala, ${ }^{11}$ Eduardo Valdivielso, ${ }^{6}$ Felipe Martínez-Alcalá, ${ }^{12}$ María Fraile, ${ }^{13}$ Alfonso Elosua, ${ }^{1}$ María Fernanda Guerra Veloz, ${ }^{7}$ Berta Ibáñez Beroiz, ${ }^{14}$ Ferrán Capdevila, ${ }^{15}$ and Mónica Enguita-Germán ${ }^{15}$

${ }^{1}$ Complejo Hospitalario de Navarra, Pamplona, Spain; ${ }^{2}$ Hospital del Mar, Barcelona, Spain; ${ }^{3}$ Hospital Universitari Mútua Terrassa, Barcelona, Spain; ${ }^{4}$ Hospital Gregorio Marañon, Madrid, Spain; ${ }^{5}$ Hospital de la Santa Creu i Sant Pau, Barcelona, Spain; ${ }^{6}$ Hospital Juan Canalejo, A Coruña, Spain; ${ }^{7}$ Hospital Universitario Virgen Macarena, Sevilla, Spain; ${ }^{8}$ Hospital Universitario Puerta de Hierro, Madrid, Spain; ${ }^{9}$ Hospital Universitario La Fe, Valencia, Spain; ${ }^{10}$ Hospital General de Ciudad Real, Ciudad Real, Spain; ${ }^{11}$ Hospital Universitario HM Montepríncipe, HM Hospitales, Madrid, Spain; ${ }^{12}$ Clinica de Gastroenterología Integral, Sevilla, Spain; ${ }^{13}$ Hospital San Pedro de Logroño, Logroño, Spain; ${ }^{14}$ Navarrabiomed, Universidad Pública de Navarra, Instituto de Investigación Sanitaria de Navarra, Red de Investigación en Servicios Sanitarios y Enfermedades Crónicas, Pamplona, Spain; and ${ }^{15}$ Navarrabiomed, Universidad Pública de Navarra, Instituto de Investigación Sanitaria de Navarra, Pamplona, Spain

This article has an accompanying continuing medical education activity, also eligible for MOC credit, on page e18 (https:// www.gastrojournal.org/cme/home). Learning Objective: Upon completion of this CME activity, successful learners will be able to assess the efficacy of clip closure of the mucosal defect after endoscopic mucosal resection (EMR) of large nonpedunculated colorectal lesions (LNPCL) that are at substantial risk of bleeding.

Multi-center RCT: 235 patients with large nonpedunculated colorectal polyps (LNPCL) with major risk of bleeding (GSEED-RE score $\geq 6$ ).

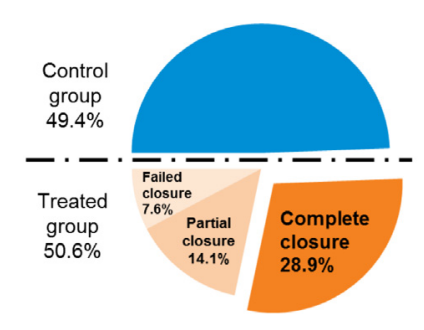

\section{See editorial on page 1190 .}

BACKGROUND \& AIMS: It is not clear whether closure of mucosal defects with clips after colonic endoscopic mucosal resection (EMR) prevents delayed bleeding, although it seems to have no protective effects when risk is low. We performed a randomized trial to evaluate the efficacy of complete clip closure of large $(\geq 2 \mathrm{~cm})$ nonpedunculated colorectal lesions after EMR in patients with an estimated average or high risk of delayed bleeding. METHODS: We performed a single-blind trial at 11 $\cup \bigcirc$ in Spain from May 2016 through June 2018, including
Delayed bleeding risk (ITT analysis)

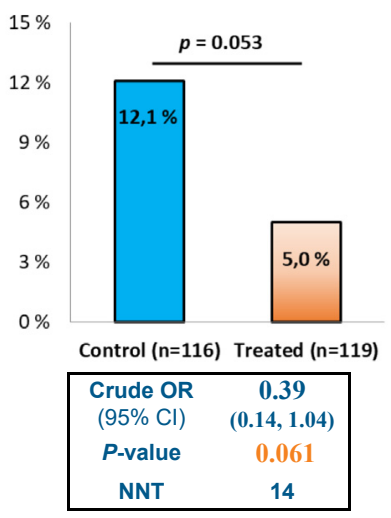

The clip closure of mucosal defects after resection of LNPCL with major risk of bleeding displays a clear trend to reduce delayed bleeding.

The protective effect is limited to those cases where complete closure was achieved.

\section{Gastroenterology}


occurred in $14(12.1 \%)$ patients in the control group and in 6 $(5 \%)$ patients in the clip group (absolute risk difference, reduction of $7 \%$ in the clip group; $95 \%$ confidence interval, $-14.7 \%$ to $0.3 \%)$. After completion of the clip closure, there was only 1 (1.5\%) case of delayed bleeding (absolute risk difference, reduction of $10.6 \%$; $95 \%$ confidence interval, $-4.3 \%$ to $17.9 \%$ ). CONCLUSIONS: In a randomized trial of patients with large nonpedunculated colorectal lesions undergoing EMR, we found that clip closure of mucosal defects in patients with a risk of bleeding can be a challenge, but also reduces delayed bleeding. Prevention of delayed bleeding required complete clip closure. ClinicalTrials.gov ID: NCT02765022.

Keywords: GSEED-RE; Polyp Removal; Complication; Prevention.

$\mathrm{D}$ elayed bleeding (DB) is defined as bleeding that occurs in a patient after discharge from the endoscopic unit, and it is the most common major complication of endoscopic mucosal resection (EMR). The mean DB rate for EMR of large $(\geq 2 \mathrm{~cm})$ nonpedunculated colorectal lesions (LNPCLs) is between $2.6 \%$ and $9.7 \% \cdot{ }^{1-5}$ DB risk has been associated with proximal polyp location, large lesions, major comorbidities, antiplatelet treatment, and patient age.,

Closure of a mucosal defect with clips after resection has long been considered to reduce the risk of bleeding. Although the application of clips after EMR is increasing, there is scarce evidence to support this practice. Several randomized controlled trials (RCTs) have shown no preventive effect of clip placement after polypectomy or EMR, even though they mostly included small polyps with a very low DB risk. ${ }^{7-10}$ A meta-analysis of these trials displayed no benefit of prophylactic clipping, but the DB risk in the control group was very low $(2.7 \%){ }^{11}$ Interestingly, one large retrospective study did show a benefit of prophylactic clipping in preventing DB in LNPCLs. ${ }^{5}$

Two large prospective cohort studies of LNPCLs ${ }^{3,6}$ have developed 2 scoring systems that allow stratification of patients into low, medium, and high DB risk groups; both studies commented on the possibility of restricting new trials to patients of at least medium DB risk to optimize resources. In addition, in the Spanish Endoscopy Society Endoscopic Resection Group (GSEED-RE) score, the absence of complete clip closure of the mucosal defect was an independent risk factor for DB.

In this RCT, we aimed to clarify the efficacy of prophylactic complete closure of mucosal defects after EMR of LNPCLs in preventing DB in selected cases with higher than average DB risk. We intended to analyze the preventive effect of complete closure with clips as both an intention-totreat (ITT) and a per-protocol (PP) analysis.

\section{Materials and Methods}

\section{Study Design}

This parallel RCT was performed in 11 Spanish hospitals by 11 experienced endoscopists from the GSEED-RE. The objective

\section{WHAT YOU NEED TO KNOW \\ BACKGROUND AND CONTEXT}

It is not clear whether closure of mucosal defects with clips after colonic endoscopic mucosal resection prevents later bleeding, although it seems to have no protective effects when risk is low.

\section{NEW FINDINGS}

In a randomized trial of patients with large nonpedunculated colorectal lesions undergoing endoscopic mucosal resection, clip closure of mucosal defects in patients with a risk of bleeding was a challenge, but also reduced bleeding within 15 days. Prevention of delayed bleeding required complete clip closure.

\section{LIMITATIONS}

Complete clip closure was not possible in $43 \%$ cases due to large size or poor accessibility.

\section{IMPACT}

Clip closure of mucosal defects should be attempted in patients with large nonpedunculated colorectal lesions with a high risk of bleeding.

was to determine the efficacy and safety of complete clip closure of mucosal defects after EMR of LNPCLs for prevention of DB.

The study protocol conformed to the ethical guidelines of the Declaration of Helsinki, received Institutional Review Board approval by the Ethics Committee at each participating center (Local code: FMSALBE), and was registered at ClinicalTrials.gov (NCT02765022). Written informed consent was obtained from all patients enrolled. All investigators who assessed the outcomes were blinded to the assignment.

\section{Study Population}

The inclusion criteria were consecutive adults over 18 years of age referred and scheduled for EMR of 1 LNPCL with substantial DB risk (GSEED-RE score $\geq 6$ ). ${ }^{3}$ This baseline was established to select polyps with higher than average estimated risk $(\geq 8 \%)$ of DB. To calculate the score, it was assumed that the variable of incomplete clip closure was initially present in all cases (Supplementary Table 1). Patients with only 1 large polyp were included in the study.

Exclusion criteria were simultaneous presence of more than 1 large $(\geq 20 \mathrm{~mm})$ polyp, lesions resected by endoscopic submucosal dissection, incomplete EMR, lesions displaying an invasive pattern, previous endoscopic attempts, suspected damage in the muscularis propria, use of adrenaline in the injection solution, and a history of a hematologic disease or coagulation disorder.

Abbreviations used in this paper: ARD, absolute risk difference; $\mathrm{Cl}$, confidence interval; DB, delayed bleeding; EMR, endoscopic mucosal resection; GSEED-RE, Spanish Endoscopy Society Endoscopic Resection Group; ITT, intention to treat; LNPCL, large nonpedunculated colorectal lesion; OR, odds ratio; PP, per protocol; TTSC, through-the-scope clip.

\section{Most current article}

(C) 2019 by the AGA Institute 0016-5085/\$36.00

https://doi.org/10.1053/j.gastro.2019.07.037 


\section{Endoscopic Mucosal Resection, Group Assignment, and Clipping Technique}

Periprocedural management with antiplatelets or anticoagulants was performed according to the European Society of Gastrointestinal Endoscopy guidelines. ${ }^{12}$ The "antiplatelet therapy" category included treatment with aspirin, clopidogrel, double antiplatelet therapy, and other anti-aggregants. Aspirin was not discontinued, but thienopyridines were routinely discontinued 5-7 days before EMR and reintroduced the day after the procedure. On the other hand, the "anticoagulant therapy" category included treatment with acenocumarol or warfarin, nonvitamin $\mathrm{K}$ oral anticoagulants, and bridging therapy with low-molecular-weight heparin. Acenocumarol or warfarin was discontinued 3-5 days before the procedure. Bridging therapy with heparin was used as recommended. Before the procedure, an international normalized ratio $<1.5$ was confirmed in patients who received anticoagulation therapy. Nonvitamin K oral anticoagulants were discontinued 48-72 hours before EMR and restarted the day after EMR.

Eleven experienced senior endoscopists ( $>100$ successful EMRs each) performed the procedures using conventional or high-definition white-light endoscopes. Lesion size was measured with either open forceps or the longitudinal edge of an open snare. Injection-assisted EMR was performed according to the guidelines in the literature. ${ }^{13}$ Adrenaline was not used in the injection solution in any case due to its possible involvement in DB. ${ }^{6}$ Erbe models (Electromedizin ICC200, VIO200, or VIO-300; Erbe, Tübingen, Germany) were used in the automatic controlled mode (Endocut-Q). Coagulation of submucosal vessels by means of snare-tip coagulation, argon plasma coagulation, or coagulation forceps was performed when the endoscopist considered it necessary. If bleeding occurred during the procedure (immediate bleeding), specific prophylactic measures on selective vessels were carried out and recorded.

Participants were randomized after EMR to a clipping or nonclipping group using a computerized sequence with a 1:1 allocation rate. Randomization assignment was automatically displayed in our web-based, password-protected program (http://clips.biotektools.org). Complete closure of the scar with 11-mm through-the-scope clips (SureClip) Micro-Tech Endoscopy (Nanjing, China) was attempted in patients assigned to the clip group. Within the treatment group, we defined 3 subgroups: complete closure, when the clips were applied next to each other and there were no substantial submucosal areas in the closure line (Figure 1); partial closure, when only a portion of the mucosal defect could be closed; and failed closure, when no part of the scar could be closed or the endoscopist considered that the mucosal defect could not be closed due to its large size or difficult location.

\section{Outcome Measures and Definitions}

The primary outcome was the DB rate after EMR, which was defined as clinically evident hematochezia that required medical attention (the patient had to contact a health care facility for an evaluation of their bleeding and required hospitalization, a blood transfusion, surgery, angiography, or repeat colonoscopy to examine the polypectomy site or to control their bleeding) within 15 days after the colonoscopy.
The secondary outcomes were other adverse events, for example, immediate bleeding, post-polypectomy syndrome, fever, and pain. Immediate bleeding refers to those episodes of hemorrhage that occurred during the procedure and lasted more than 30 seconds or required endoscopic treatment. Postpolypectomy syndrome was defined by symptoms of pain, fever, leukocytosis, peritoneal tenderness, and guarding. Fever was considered when a patient presented with a temperature $>38^{\circ} \mathrm{C}$ after polypectomy with no evidence of other explainable fever foci. Finally, pain was considered when a patient required pharmacologic treatment or medical attention.

\section{Data Collection}

Demographic data were collected from a personal interview and from medical charts. The variables related to the lesion characteristics and EMR procedures were collected in the endoscopy suite. Participants were contacted by telephone or a personal interview 15 days after EMR. Follow-up was performed at the outpatient clinic of each center. The study data were recorded in an online password-protected database.

\section{Statistical Analysis}

An initial sample size of 332 patients was calculated to be needed to reduce the DB risk from $8 \%$ (the lowest allowed) to $2 \%$ in a bilateral test, assuming an $\alpha$ value of 5\%, a statistical power of $80 \%$, and an expected attrition rate of $2 \%$. However, other possible sample sizes were considered, depending on the observed DB rates in the control group. After some researchers voiced concerns about high DB rates in their cohorts, an unplanned interim analysis was conducted of the study by a safety review committee when 200 patients had been recruited. The primary investigator was blinded to the results. A new sample size of 227 participants was recalculated to reduce the DB risk from $10 \%$ to $2 \%$ in a bilateral test, assuming a statistical power of $80 \%$ and a final $\alpha$ value of $4.92 \%$ (following the O'Brien Fleming $\alpha$-spending approach) for 1 interim analysis. The study was finally stopped when the sample consisted of 235 patients.

The ITT analysis included all randomized cases. The PP analysis included only the cases in which complete mucosal defect closure was achieved. A post-hoc analysis of the variables related with complete closure, partial closure, and failed closure was also conducted.

The characteristics of the sample were described using descriptive statistics; for categorical variables, frequencies and percentages were used, and for quantitative variables, means and SDs were used. Comparisons between groups were assessed using Student $t$ tests or analysis of variance for continuous variables and with $\chi^{2}$ test or Fisher's exact test for categorical variables. The incidence of DB within each treatment group was estimated using sample proportions with $95 \%$ confidence intervals (CIs). Univariate logistic regression models were used to estimate the crude odds ratios (ORs) for DB in the treatment groups. As a secondary end point, we aimed to identify the risk factors associated with DB. Multivariate logistic regression models included the treatment group and other covariates that were significant in the univariate models, providing the adjusted ORs. Statistical significance was considered as $P<.049$. Statistical analysis was performed using IBM SPSS Statistics software, version 25.0 (IBM Corp, 

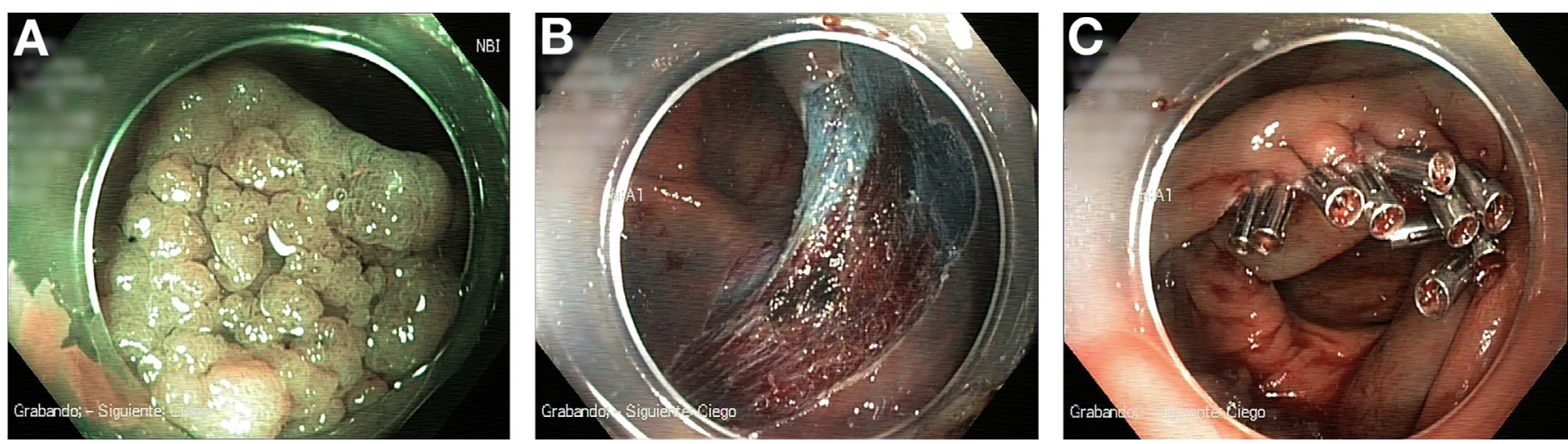

Figure 1. Complete closure of an LNPCL. (A) Mixed granular adenoma. (B) Mucosal defect after EMR. (C) Complete closure of the mucosal defect using hemoclips.

Armonk, NY) and R 3.1.0 ( $\mathrm{R}$ Foundation for Statistical Computing, Vienna, Austria; http://www.R-project.org/).

All authors had access to the study data and reviewed and approved the final manuscript.

\section{Results}

\section{Patients}

Between May 2016 and June 2018, 237 patients with LNPCLs were recruited into the study. Two patients were excluded for delayed perforation, 1 in each group. There were 116 patients in the control group and 119 in the clip group. A flowchart is shown in Supplementary Figure 1.

Comparing the baseline characteristics between the control and treatment groups, there was an imbalance in the antiplatelet treatment that was more frequently used in the clip group. There were no other differences between patient characteristics or LNPCL features (Table 1).

In the clip group, complete closure was achieved in 68 (57\%) patients and partial closure was achieved in 33 $(28 \%)$ patients. Failed closure occurred in the remaining 18 (15\%) patients. Median number of clips necessary for complete closure was 6 (range, 3-13). Comparing the baseline characteristics between the control group and complete closure subgroup, there were more patients aged 75 years and older and the polyps were smaller in the complete closure subgroup (data not shown).

Snare-tip coagulation was used in 44 (38\%) cases in the control group and in $50(42 \%)$ cases in the clip group $(P=.61)$. Argon plasma coagulation was used in $9(8 \%)$ cases in the control group and in $10(8 \%)$ cases in the clip group $(P=.94)$. Coagulation forceps were used in $2(2 \%)$ cases in the control group and in $3(3 \%)$ cases in the clip group $(P=1)$. In the control group, 9 patients $(8 \%)$ required punctual clipping due to immediate bleeding and none of the cases received a complete closure.

\section{Primary Outcome}

Of the total 235 patients, DB occurred in 20 (8.5\%) patients. There were $14(12.1 \%)$ DB events in the control group and $6(5 \%)$ in the clip group (Table 2). Seven cases occurred in the first 48 hours, 8 cases occurred between 3 and 7 days post-EMR, and 5 cases were in the second week after the resection.

For control of DB, no patient required a surgical or an angiographic intervention. Additional endoscopy was necessary in 15 of $20(75 \%)$ patients: 2 patients in the clip group were treated with 2 hemostatic methods, 8 patients in the control group also received endoscopic intervention (7 patients treated with clips and 1 with 2 methods), and 5 patients did not receive any treatment. Four patients required transfusions. The 20 cases of DB accounted for a total of 61 days of hospitalization (median, 3 days; range, 1-7 days).

In the ITT analysis, the DB risk was $12.1 \%$ in the control group and $5 \%$ in the clip group (Table 2 and Figure 2), with an absolute risk difference (ARD) of $-7 \%$ (95\% CI, $-14.7 \%$ to $0.3 \% ; P=.053$ ) and crude OR of 0.39 . The number needed to treat was 14 . To complement the main result of the ITT analysis, a multivariate analysis adjusting for the confounding variable "antiplatelet therapy" was performed, obtaining an adjusted OR of $0.30(P=.022)$. In the PP analysis, the DB risk was $12.1 \%$ in the control group and $1.5 \%$ in the complete closure group (Table 2 and Figure 2), with an ARD of $-10.6 \%(95 \% \mathrm{CI},-17.9 \%$ to $-2.6 \%$; $P=.01)$ and a crude OR of 0.11 .

A post-hoc analysis of the primary outcome in cases grouped according to several risk factors was performed (Supplementary Table 2).

The effect of clip closure was also analyzed stratifying the lesions based on the proximal or distal location. Two different definitions of proximal location (with or without transverse colon) were included in the analysis (Supplementary Table 3).

\section{Clipping Efficacy Analysis}

The DB risk among the subgroups of the clip group was $1.5 \%$ in the complete closure subgroup, $9.1 \%$ in the partial closure subgroup, and $11 \%$ in the failed closure subgroup.

The polyps that were successfully clipped were smaller, had better accessibility, and had shorter and easier EMR procedures (Supplementary Table 4). Accessibility and difficulty of EMR were subjective criteria chosen by the endoscopist. Other polyp characteristics, such as exact location, histology, or morphologic classification, were not related to complete closure failure in our cohort. 
Table 1.Baseline Characteristics of Patients From the Intervention Groups

\begin{tabular}{|c|c|c|c|}
\hline Variable & $\begin{array}{c}\text { Control group, } \\
(\mathrm{n}=116 \text { [49.4\%]) }\end{array}$ & $\begin{array}{l}\text { Treatment group, } \\
(\mathrm{n}=119 \text { [50.6\%]) }\end{array}$ & $P$ value \\
\hline \multicolumn{4}{|l|}{ Demographic characteristics } \\
\hline Age group & & & .071 \\
\hline$<75$ y & $73(62.9)$ & $61(51.3)$ & \\
\hline$\geq 75$ y & $43(37.1)$ & $58(48.7)$ & \\
\hline Female & $39(33.6)$ & 38 (31.9) & \\
\hline \multicolumn{4}{|l|}{ Clinical characteristics } \\
\hline ASA class & & & .154 \\
\hline I & $9(7.8)$ & $8(6.7)$ & \\
\hline II & $49(42.2)$ & $39(32.8)$ & \\
\hline III & $56(48.3)$ & $69(58.0)$ & \\
\hline Hypertension & & & .335 \\
\hline No & $44(37.9)$ & $38(31.9)$ & \\
\hline Yes & $72(62.1)$ & $81(68.1)$ & \\
\hline Antiplatelet therapy & & & $.042^{d}$ \\
\hline No & $82(70.7)$ & $69(58)$ & \\
\hline Yes & $34(29.3)$ & $50(42.0)$ & \\
\hline \multicolumn{4}{|l|}{ Anticoagulant therapy } \\
\hline No & $85(73.3)$ & $89(74.8)$ & .791 \\
\hline Yes & $31(26.7)$ & $30(25.2)$ & \\
\hline Heparin bridge therapy ${ }^{a}$ & & & .947 \\
\hline No & $6(19.4)$ & 7 (23.3) & \\
\hline Yes & $25(80.6)$ & $23(76.7)$ & \\
\hline Proximal location ${ }^{b}$ (including TC) & & & .609 \\
\hline No & $12(10.3)$ & $10(8.4)$ & \\
\hline Yes & $104(89.7)$ & $109(91.6)$ & \\
\hline Proximal location ${ }^{c}$ (excluding TC) & & & .967 \\
\hline No & $28(24.1)$ & $29(24.4)$ & \\
\hline Yes & $88(75.9)$ & $90(75.6)$ & \\
\hline Histology & & & .413 \\
\hline Adenoma-LGD & $68(58.6)$ & $72(60.5)$ & \\
\hline Adenoma-HGD & $36(31.0)$ & $35(29.4)$ & \\
\hline Adenocarcinoma-SI & $5(4.3)$ & $4(3.4)$ & \\
\hline Serrated/serrated-LGD & $7(6.0)$ & $4(3.4)$ & \\
\hline Serrated-HGD & $0(0.0)$ & $3(2.5)$ & \\
\hline Serrated carcinoma-SI & $0(0.0)$ & $1(0.8)$ & \\
\hline High-risk histology & & & .743 \\
\hline No & $71(63.4)$ & $68(61.3)$ & \\
\hline Yes & $41(36.6)$ & $43(38.7)$ & \\
\hline
\end{tabular}

NOTE. Values are $\mathrm{n}(\%)$ unless otherwise specified.

ADC, adenocarcinoma; ASA, American Society of Anesthesiologists; HGD, high-grade dysplasia; LGD, low-grade dysplasia; $\mathrm{SI}$, superficial invasion; TC, transverse colon.

${ }^{a}$ Described only for patients who received the chronic anticoagulant treatment.

${ }^{b}$ Location from cecum to the TC (included).

${ }^{c}$ Location from cecum to the TC (excluded).

${ }^{d}$ Significant $(P<.05)$. 
Table 2. Primary and Secondary Outcomes From the Intention-to-Treat ${ }^{a}$ and Per-Protocol ${ }^{b}$ Analyses

\begin{tabular}{|c|c|c|c|}
\hline Variable & Control group & Intervention group & $P$ value \\
\hline \multicolumn{4}{|l|}{ DB } \\
\hline ITT, n (\%) & $14(12.1)$ & $6(5.0)$ & .053 \\
\hline Crude OR (95\% Cl) & Reference & $0.39(0.14-1.04)$ & .061 \\
\hline $\mathrm{PP}, \mathrm{n}(\%)$ & $14(12.1)$ & $1(1.5)$ & $.011^{c}$ \\
\hline Crude OR $(95 \% \mathrm{Cl})$ & Reference & $0.11(0.01-0.85)$ & $.034^{c}$ \\
\hline \multicolumn{4}{|l|}{ Immediate bleeding } \\
\hline ITT, n (\%) & $18(15.5)$ & $21(17.6)$ & .661 \\
\hline Crude OR $(95 \% \mathrm{Cl})$ & Reference & $1.17(0.59-2.32)$ & .661 \\
\hline PP, n (\%) & $18(15.5)$ & $10(14.7)$ & .882 \\
\hline Crude OR (95\% Cl) & Reference & $0.94(0.41-2.17)$ & .882 \\
\hline \multicolumn{4}{|c|}{ Post-polypectomy syndrome } \\
\hline ITT, n (\%) & $0(0)$ & $3(2.5)$ & .247 \\
\hline $\mathrm{PP}, \mathrm{n}(\%)$ & $0(0)$ & $1(1.5)$ & .370 \\
\hline \multicolumn{4}{|l|}{ Perforation } \\
\hline ITT, n (\%) & $1(0.9)$ & $1(0.8)$ & .999 \\
\hline $\mathrm{PP}, \mathrm{n}(\%)$ & $1(0.9)$ & $0(0)$ & .999 \\
\hline \multicolumn{4}{|l|}{ Fever } \\
\hline ITT, n (\%) & $1(0.9)$ & $1(0.8)$ & .999 \\
\hline PP, n (\%) & $1(0.9)$ & $0(0)$ & .999 \\
\hline \multicolumn{4}{|l|}{ Pain } \\
\hline ITT, n (\%) & $2(1.7)$ & $6(5.0)$ & .281 \\
\hline $\mathrm{PP}, \mathrm{n}(\%)$ & $2(1.7)$ & $2(2.9)$ & .627 \\
\hline
\end{tabular}

${ }^{a}$ Control vs treated.

${ }^{b}$ Control vs complete closure.

${ }^{c}$ Significant $(P<.05)$.

\section{Other Adverse Events}

Immediate bleeding occurred in $18(16 \%)$ patients and $21(18 \%)$ patients in the control and clip groups, respectively $(P=.79)$, and it was mainly managed with snare softtip coagulation. Four cases with immediate bleeding also developed DB. There were no cases of post-polypectomy syndrome in the control group and 3 cases in the clip group $(P=.25)$. Of these 3 cases, the endoscopist achieved complete clip closure in 1 and failed in the other 2 cases. All cases evolved favorably with analgesia and broad-spectrum antibiotics. Only 2 patients had post-polypectomy fever (1 patient in each group). Finally, 2 (2\%) patients in the control group and $6(5 \%)$ patients in the clip group complained of pain that required medical intervention $(P=.28)$. There were no deaths.

\section{Discussion}

In this multicenter controlled trial, clip closure of mucosal defects after resection of LNPCLs with substantial

\section{Delayed bleeding risk} (ITT analysis)

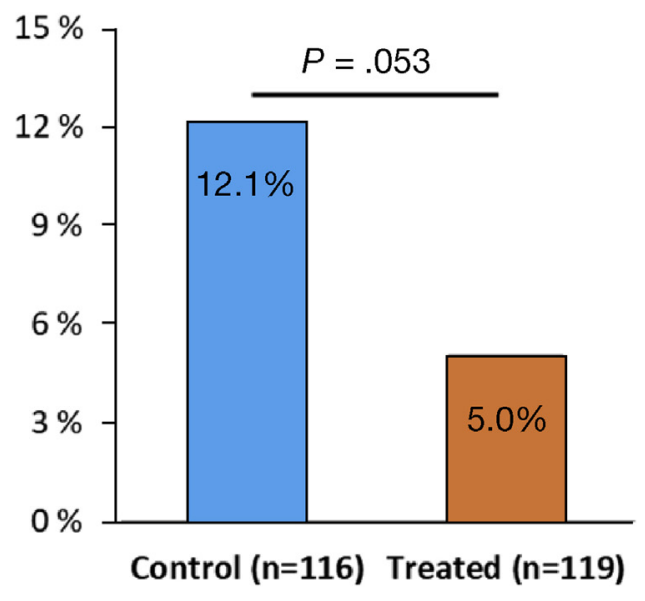

Delayed bleeding risk (PP analysis)

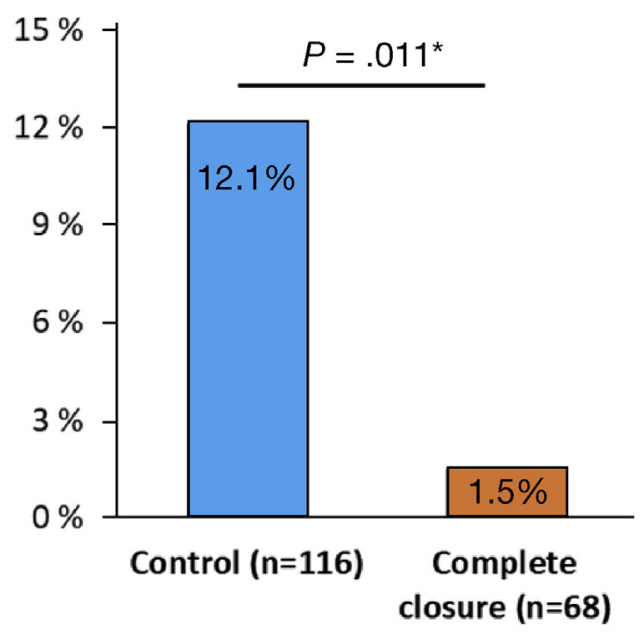

Figure 2. DB risk in the ITT analysis (control vs treated) and in the PP analysis (control vs complete closure). 
risk of bleeding displayed a clear trend to reduce DB risk. An evident protective effect was observed in those cases where complete clip closure was achieved.

Clinically significant DB is the most frequent severe adverse event of EMR after resection of large colonic lesions. Several techniques to prevent DB have been described. Adrenaline injection into the submucosal tissue prevents immediate bleeding, but it is not useful for treating DB. ${ }^{14}$ Coagulation techniques, such as argon plasma coagulation (APC) and the use of coagulation forceps, had no preventive effect in 2 RCTs. ${ }^{15,16}$ The most common practice to prevent DB is closure of the mucosal defect with TTSCs.

Several RCTs have analyzed the closure of mucosal defects with TTSCs. ${ }^{7-10,17}$ A meta-analysis of those trials showed no benefit of preventive clipping, with a DB rate of $2.2 \%$ in the clip group vs $2.7 \%$ in the control group. ${ }^{11}$ However, that meta-analysis and the RCTs that compose it were not specifically designed to evaluate DB in LNPCLs and had several other limitations, such as the low risk of DB in small lesions, the heterogeneous morphology of the polyps, the imprecise definition of $\mathrm{DB}$, and the difference in resection techniques used. ${ }^{17}$

A large retrospective study in a single hospital including only LNPCLs showed a reduction in the bleeding risk of $10 \%$ in the nonclipping group and $1.8 \%$ in the fully clipped group. ${ }^{5}$ Despite the limitations of this uncontrolled study, a clear benefit of clip closure in preventing DB in cases with large mucosal defects was shown, especially when the clip closure was complete.

Our group also showed, in an observational multicenter study with a large sample (1214 cases), that prophylactic clipping was an independent protective factor against $\mathrm{DB}{ }^{3}$

Recently, a large trial showed a benefit from prophylactic clipping for any LNPCL $\geq 20 \mathrm{~mm}^{18}$ Although the population included was very large, some polyps (such as distal polyps) had a low DB risk, resulting in a high number needed to treat $(n=28)$. A proximal location, large size, presence of major comorbidities, and antiplatelet or anticoagulant treatment have been recognized as independent risk factors for DB in 2 large observational studies. ${ }^{3,6}$ Proximal location was the variable with the highest weight in the GSEED-RE score. Accordingly, proximal location was the only variable that demonstrated a statistically significant benefit in the trial conducted by Pohl et al. ${ }^{18}$ Even though there was a greater DB risk with increased polyp size and in patients on antiplatelet medication, the study did not show a benefit of clipping in those patients; there was no attempt to analyze patients with a combination of risk factors. Furthermore, no DB risk assessment was performed before inclusion and, accordingly, the DB risk of the trial population depended on the relative proportion of high and low DB risk cases. By contrast, our study included only lesions with substantial DB risk ( $\geq 8 \%$ ). More than $90 \%$ of the cases were proximal polyps and $51 \%$ were $>40 \mathrm{~mm}$; in addition, $36 \%$ of the patients were on antiplatelets. We combined those variables using the GSEED-RE score, resulting in a DB occurrence of $12.1 \%$ in the control group, which was higher than reported in other studies.
In our selected population, polyps were markedly large (mean size of $36.6 \mathrm{~mm}$ ). This fact explained the increased number of high-risk histology lesions and had a negative effect on the success of complete closure. Even so, our trial showed a clear trend of clip closure to reduce DB risk in the ITT analysis, with an ARD of $7 \%(P=.053)$. The association reached statistical significance after adjusting for the antiplatelet therapy variable (more frequent in the clip group). The protective effect of clip closure was stronger when complete closure of the mucosal defect was achieved, with an ARD of 10.6\%; however, due to the low number of DB cases in the complete closure group, this risk difference might be highly susceptible to variations in the number of events.

The analysis of the subgroup of cases based on the location of the polyps deserves special attention. We considered 2 definitions of proximal location, 1 including the transverse colon (as in the GSEED-RE risk score) and another excluding this colonic segment. With both definitions the results were similar. In addition, the incidence of DB in the transverse colon was very low; among the 35 transverse colon polyps there was only 1 DB case in the intervention group (failed closure) and no cases in the control group. In a validation study of the GSEED-RE risk score, excluding the transverse colon from the proximal location yielded better results and could be more appropriate for selecting high-risk bleeding polyps in future studies. ${ }^{19}$ In the subgroup of proximal polyps, there was a trend of a protective effect of clip closure, and the association was statistically significant when the clip closure was complete. The distal polyps were always underrepresented, and no solid conclusion could be drawn about them.

Our study has several strengths. We included only LNPCLs with higher than average estimated DB risk. Other studies including low-risk polyps failed to show a preventive effect of clipping ${ }^{7-10,20}$ or had an number needed to treat that was too large. ${ }^{18}$ We only included cases with the EMR technique, reflecting the clinical practice of Western countries. The participation of several hospitals makes the results more generalizable to a real-world setting. Additionally, there was no crossover of treatments. Finally, the inclusion of only 1 large polyp per patient eliminated the possibility of bleeding at another polyp resection site.

This study has some limitations. The inclusion criterion of a GSEED-RE score of $\geq 6$ points was made based on an uncontrolled observational study. Although this trial showed a clear benefit for that choice, it is possible that another cutoff or the use of our updated risk score version ${ }^{19}$ would better select cases in which clipping is most beneficial. The incidence of $\mathrm{DB}$ in our selected population was difficult to predict and affected the sample size calculation. The timing of randomization is a controversial issue. Randomization after completing the EMR may prevent a potentially more intensive application of other DB preventive measures in the control group, but it may introduce a selection bias toward excluding mucosal defects difficult to close with clips. Additionally, information about the excluded polyps, which could have been relevant for the 
generalization of the results, was not collected. In any case, the mean polyp size of this study was larger than previous publications and, accordingly, the rate of complete closure was lower than other studies, therefore, the number of polyps excluded for being difficult to clip was probably low. The clipping efficacy analysis was limited because polyps for which complete closure was achieved were smaller and with better accessibility than polyps with partial or failed closure, and those variables may have influenced the DB risk. Finally, complete clip closure was not possible in $43 \%$ of the cases. As we selected cases with a higher than average estimated risk, half of the lesions were $\geq 40 \mathrm{~mm}$, and those mucosal defects were more difficult to completely close. In our trial, only 11-mm Microtech TTSCs were used for standardization of the clipping technique. Currently, it is possible to choose more suitable TTSCs, depending on the mucosal defect size (up to $16 \mathrm{~mm}$ ). ${ }^{21}$ Additionally, advanced suturing techniques with TTSCs have been described, such as a silk loop, ${ }^{22}$ a line-assisted closure, ${ }^{23}$ or a clip-fixed endoloop. ${ }^{24}$ These techniques are not widely implemented, and our multicenter trial reflects that it is not always easy to achieve complete closure with TTSCs. In any case, clipping the defect is worth an attempt, as there was a clear risk reduction in the clip group, although this reduction was due mainly to the complete closure subgroup. We may consider that disposing of different TTSCs and mastering advanced clipping techniques could become a necessity when treating difficult lesions.

In summary, this multicenter clinical trial found that complete clip closure of mucosal defects after resection of large colon lesions, in selected patients with higher than average estimated DB risk, can be a challenging technique but displays a clear trend toward reducing DB risk. The protective effect is limited to cases where complete clip closure is achieved.

\section{Supplementary Material}

Note: To access the supplementary material accompanying this article, visit the online version of Gastroenterology at www.gastrojournal.org, and at https://doi.org/10.1053/ j.gastro.2019.07.037.

\section{References}

1. Hassan C, Repici A, Sharma P, et al. Efficacy and safety of endoscopic resection of large colorectal polyps: a systematic review and meta-analysis. Gut 2016;65:806-820.

2. Burgess NG, Metz AJ, Williams SJ, et al. Risk factors for intraprocedural and clinically significant delayed bleeding after wide-field endoscopic mucosal resection of large colonic lesions. Clin Gastroenterol Hepatol 2014; 12:651-661 e1-e3.

3. Albéniz E, Fraile M, Ibañez B, et al. A scoring system to determine risk of delayed bleeding after endoscopic mucosal resection of large colorectal lesions. Clin Gastroenterol Hepatol 2016;14:1140-1147.
4. Buchner AM, Guarner-Argente C, Ginsberg GG. Outcomes of EMR of defiant colorectal lesions directed to an endoscopy referral center. Gastrointest Endosc 2012; 76:255-263.

5. Liaquat H, Rohn E, Rex DK. Prophylactic clip closure reduced the risk of delayed postpolypectomy hemorrhage: experience in 277 clipped large sessile or flat colorectal lesions and 247 control lesions. Gastrointest Endosc 2013;77:401-407.

6. Bahin FF, Rasouli KN, Byth K, et al. Prediction of clinically significant bleeding following wide-field endoscopic resection of large sessile and laterally spreading colorectal lesions: a clinical risk score. Am J Gastroenterol 2016;111:1115-1122.

7. Shioji K, Suzuki Y, Kobayashi M, et al. Prophylactic clip application does not decrease delayed bleeding after colonoscopic polypectomy. Gastrointest Endosc 2003; 57:691-694.

8. Dokoshi T, Fujiya M, Tanaka K, et al. A randomized study on the effectiveness of prophylactic clipping during endoscopic resection of colon polyps for the prevention of delayed bleeding. Biomed Res Int 2015; 2015:490272.

9. Mori H, Kobara H, Nishiyama N, et al. Simple and reliable treatment for post-EMR artificial ulcer floor with snare cauterization for $10-$ to $20-\mathrm{mm}$ colorectal polyps: a randomized prospective study (with video). Surg Endosc 2015;29:2818-2824.

10. Matsumoto M, Kato M, Oba K, et al. Multicenter randomized controlled study to assess the effect of prophylactic clipping on post-polypectomy delayed bleeding. Dig Endosc 2016;28:570-576.

11. Nishizawa T, Suzuki H, Goto O, et al. Effect of prophylactic clipping in colorectal endoscopic resection: a meta-analysis of randomized controlled studies. United European Gastroenterol J 2017;5:859-867.

12. Veitch AM, Vanbiervliet G, Gershlick AH, et al. Endoscopy in patients on antiplatelet or anticoagulant therapy, including direct oral anticoagulants: British Society of Gastroenterology (BSG) and European Society of Gastrointestinal Endoscopy (ESGE) guidelines. Gut 2016;65:374-389.

13. Albéniz E, Pellisé M, García AZG, et al. Clinical guidelines for endoscopic mucosal resection of non-pedunculated colorectal lesions. Gastroenterol Hepatol 2018;41:175190.

14. Lee S-H. Comparison of postpolypectomy bleeding between epinephrine and saline submucosal injection for large colon polyps by conventional polypectomy: a prospective randomized, multicenter study. World J Gastroenterol 2007;13:2973-2977.

15. Lee CK, Lee SH, Park JY, et al. Prophylactic argon plasma coagulation ablation does not decrease delayed postpolypectomy bleeding. Gastrointest Endosc 2009; 70:353-361.

16. Bahin FF, Naidoo M, Williams SJ, et al. Prophylactic endoscopic coagulation to prevent bleeding after widefield endoscopic mucosal resection of large sessile 
colon polyps. Clin Gastroenterol Hepatol 2015;13:724730. e1-e2.

17. Zhang QS, Han B, Xu JH, et al. Clip closure of defect after endoscopic resection in patients with larger colorectal tumors decreased the adverse events. Gastrointest Endosc 2015;82:904-909.

18. Pohl H, Grimm IS, Moyer MT, et al. Clip closure prevents bleeding after endoscopic resection of large colon polyps in a randomized trial. Gastroenterology 2019 Mar 15. pii: S0016-5085(19)33573-33575.

19. Albéniz E, Fraile M, Zebenzuy A, et al. Development of a new score system to determine the risk of delayed bleeding after endoscopic mucosal resection of large colorectal lesions. Endoscopy 2018;50:S67.

20. Feagins LA, Nguyen AD, Iqbal $R$, et al. The prophylactic placement of hemoclips to prevent delayed postpolypectomy bleeding: an unnecessary practice? A case control study. Dig Dis Sci 2014;59:823-828.

21. Wang TJ, Aihara H, Thompson AC, et al. Choosing the right through-the-scope clip: a rigorous comparison of rotatability, whip, open/close precision, and closure strength (with videos). Gastrointest Endosc 2019;89:77-86 e71.

22. Wang W, Jin L, Zhao F, et al. Endoscopic closure of the defect after endoscopic submucosal resection with metal clips and surgical sutures. VideoGIE 2019;4: 22-23.

23. Yamasaki $Y$, Takeuchi $Y$, Kato $M$, et al. Line-assisted complete closure of large gastric mucosal defects by use of multiple clip-and-line technique. VideoGIE 2016;1: $49-50$.

24. Nomura T, Kobayashi M, Morikawa T, et al. Clip-fixed endoloop: an efficacious new method for mucosal defect closure. Endoscopy 2018;50:E126-E127.
Received April 11, 2019. Accepted July 21, 2019.

\section{Reprint requests}

Address requests for reprints to: Eduardo Albéniz, MD, Navarrabiomed Biomedical Research Center, Universidad Pública de Navarra, Instituto de Investigación Sanitaria de Navarra, Endoscopy Unit, Gastroenterology Department, Complejo Hospitalario de Navarra, Irunlarrea 3, 31008, Navarra Spain. e-mail: edualbeniz@hotmail.com; fax: 848422200.

\section{Acknowledgments}

The authors would like to acknowledge the Spanish Society of Digestive Endoscopy and the members of the GSEED-RE for their continued anonymous and tireless collaboration within this working group, and the Navarrabiomed-Miguel Servet Foundation for the technical management of the project and the statistical support. English editing and manuscript formatting were performed by American Journal Experts, LLC (Durham, NC).

Author contributions: Eduardo Albéniz designed the study, performed the procedures, collected data, analyzed data, and wrote the manuscript. Marco Antonio Álvarez performed procedures, collected data, and wrote the manuscript. Jorge C. Espinós performed procedures and collected data. Oscar Nogales performed procedures and collected data. Carlos Guarne performed procedures and collected data. Pedro Alonso performed procedures and collected data. Manuel Rodríguez-Téllez performed procedures and collected data. Alberto Herreros de Tejada performed procedures and collected data. José Santiago collected data. Marco Bustamante-Balén performed procedures and collected data. Joaquín Rodríguez Sánchez performed procedures and collected data. Felipe Ramos-Zabala performed procedures and collected data. Eduardo Valdivielso collected data. Felipe Martínez-Alcalá collected data. María Fraile analyzed data. Alfonso Elosua collected data and analyzed data. María Fernanda Guerra Veloz collected data. Ferrán Capdevila designed the study. Berta Ibáñez Beroiz analyzed data and wrote the manuscript. Mónica Enguita-Germán analyzed data and wrote the manuscript. Marco Antonio Álvarez and Jorge C. Espinós contributed equally to this work.

\section{Conflicts of interest}

The authors disclose no conflicts.

Funding

This work was supported by a grant from the Spanish Society of Digestive Endoscopy. Micro-Tech Endoscopy (Nanjing, China) contributed the clips needed for the study. The authors declare no conflicts of interest with this company or any other that relates to the topic addressed in this study. The first author received a grant from "La Caixa/Caja Navarra" Foundation (ID 100010434; Project PR15/11100006). 


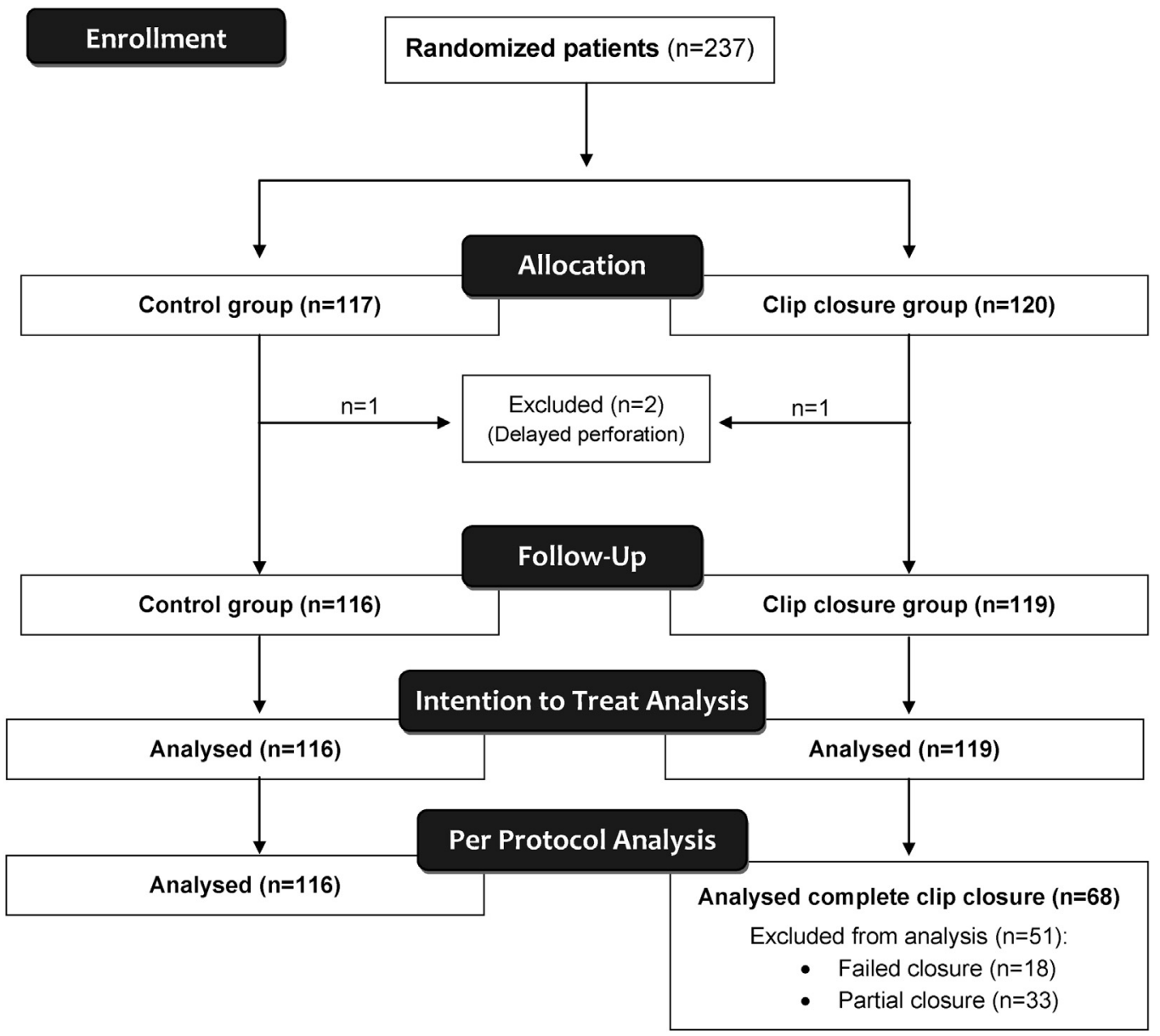

Supplementary Figure 1. Flow chart of patients/polyps in the RCT.

Supplementary Table 1.Spanish Endoscopy Society

Endoscopic Resection Group

Delayed Bleeding Score of Large

Nonpedunculated Colorectal

Lesions

\begin{tabular}{lc}
\hline \multicolumn{1}{c}{ Variable } & Score \\
\hline Proximal location (cecum to transverse, included) & 3 \\
Antiplatelet treatment & 2 \\
Lesion size $\geq 40 \mathrm{~mm}$ & 1 \\
ASA class III-IV & 1 \\
Age $\geq 75$ y & 1 \\
No complete clip closure of the mucosal defect & 2
\end{tabular}

ASA, American Society of Anesthesiologists. 
Supplementary Table 2. Analysis of Delayed Bleeding by Subgroups of Risk Factors

\begin{tabular}{|c|c|c|c|c|}
\hline Variable & $\begin{array}{l}\text { Control, n/N (\%) } \\
\quad(n=116)\end{array}$ & $\begin{array}{l}\text { Treated, } n / N(\%) \\
\quad(n=119)\end{array}$ & $P$ value & ARD $(95 \% \mathrm{Cl})$ \\
\hline \multicolumn{5}{|l|}{ Size } \\
\hline$\geq 40 \mathrm{~mm}$ & $5 / 62(8.1)$ & $1 / 53(1.9)$ & .215 & $-6.2(-13.8$ to 1.53$)$ \\
\hline \multicolumn{5}{|l|}{ ASA group } \\
\hline $\mathrm{ASA} I+\|$ & $4 / 58(6.9)$ & $1 / 47(2.1)$ & .377 & $-4.8(-12.5$ to 2.95$)$ \\
\hline \multicolumn{5}{|c|}{ Proximal location ${ }^{a}$} \\
\hline No & $3 / 12(20.0)$ & $1 / 10(10.0)$ & .594 & $-15(-45.7$ to 15.7$)$ \\
\hline Yes & 11/104 (10.6) & $5 / 109(4.6)$ & .097 & $-6(-13.1$ to 1.11$)$ \\
\hline \multicolumn{5}{|c|}{ Antiplatelet therapy } \\
\hline No & 6/82 (7.3) & 1/69 (1.4) & .126 & $-5.87(-12.2$ to 0.43$)$ \\
\hline Yes & $8 / 34(23.5)$ & $5 / 50(10)$ & .092 & $-13.5(-30.0$ to 2.97$)$ \\
\hline \multicolumn{5}{|l|}{ Age $\geq 75$} \\
\hline
\end{tabular}

ASA, American Society of Anesthesiologists.

${ }^{a}$ Location from cecum to the transverse colon (included).

Supplementary Table 3. Delayed Bleeding in Patients From the Intention-to-Treat and Per-Protocol Analyses Between Proximal and Distal Lesions

\begin{tabular}{|c|c|c|c|c|c|c|}
\hline \multirow[b]{3}{*}{ Variable } & \multicolumn{6}{|c|}{ TC included in the proximal location definition } \\
\hline & \multicolumn{3}{|c|}{ Proximal polyps $(n=213$ ) } & \multicolumn{3}{|c|}{ Distal polyps $(\mathrm{n}=22)$} \\
\hline & Control group & Intervention group & $P$ value & Control group & Intervention group & $P$ value \\
\hline \multicolumn{7}{|l|}{$\mathrm{ITT}$} \\
\hline $\mathrm{n} / \mathrm{N}(\%)$ & 11/104 (10.6) & $5 / 109(4.6)$ & .097 & $3 / 12(25)$ & $1 / 10(10)$ & .594 \\
\hline Crude OR & Reference & $0.41(0.14-1.21)$ & .107 & Reference & $0.33(0.03-3.84)$ & .378 \\
\hline \multicolumn{7}{|l|}{$\mathrm{PP}$} \\
\hline $\mathrm{n} / \mathrm{N}(\%)$ & $11 / 104(10.6)$ & $1 / 68(1.6)$ & $.031^{a}$ & 3/12 (25) & 0/6 (0) & .515 \\
\hline \multirow[t]{3}{*}{ Crude OR } & Reference & $0.14(0.02-1.1)$ & .062 & - & - & - \\
\hline & \multicolumn{6}{|c|}{ TC excluded from the proximal location definition } \\
\hline & \multicolumn{3}{|c|}{ Proximal polyps $(n=178)$} & \multicolumn{3}{|c|}{ Distal polyps $(n=57)$} \\
\hline Variable & Control group & Intervention group & $P$ value & Control group & Intervention group & $P$ value \\
\hline \multicolumn{7}{|l|}{$\mathrm{ITT}$} \\
\hline $\mathrm{n} / \mathrm{N}(\%)$ & 11/88 (12.5) & 4/90 (4.4) & .053 & 3/28 (10.7) & 2/29 (6.9) & .670 \\
\hline Crude OR & Reference & $0.33(0.10-1.06)$ & .063 & Reference & $0.62(0.09-4.01)$ & .613 \\
\hline \multicolumn{7}{|l|}{$\mathrm{PP}$} \\
\hline $\mathrm{n} / \mathrm{N}(\%)$ & $11 / 88(12.5)$ & $1 / 51(2.0)$ & $.033^{a}$ & $3 / 28(10.7)$ & $0 / 17(0)$ & .279 \\
\hline Crude OR & Reference & $0.14(0.02-1.1)$ & .064 & - & - & - \\
\hline
\end{tabular}

TC, transverse colon.

asignificant $(P<.05)$. 
Supplementary Table 4. Clipping Efficacy Analysis

\begin{tabular}{|c|c|c|c|c|}
\hline Variable & $\begin{array}{l}\text { Failed closure, } \\
(\mathrm{n}=18[15.1 \%])\end{array}$ & $\begin{array}{l}\text { Partial closure, } \\
(\mathrm{n}=33[27.7 \%])\end{array}$ & $\begin{array}{l}\text { Complete closure, } \\
(\mathrm{n}=68[57.1 \%])\end{array}$ & $P$ value \\
\hline \multicolumn{5}{|l|}{ Demographic characteristics } \\
\hline Age group & & & & .198 \\
\hline$<75$ y & $11(18.0)$ & $20(32.8)$ & $30(49.2)$ & \\
\hline$\geq 75$ y & 7 (12.1) & $13(22.4)$ & $38(65.5)$ & \\
\hline Female & $6(15.8)$ & $12(31.6)$ & $20(52.6)$ & \\
\hline \multicolumn{5}{|l|}{ Clinical characteristics } \\
\hline ASA class & & & & .592 \\
\hline I & $0(0)$ & $4(50.0)$ & $4(50.0)$ & \\
\hline ॥ & $8(20.5)$ & $10(25.6)$ & $21(53.8)$ & \\
\hline III & $10(15.4)$ & $18(26.1)$ & $41(59.4)$ & \\
\hline Antiplatelet/anticoagulant therapy & & & & .445 \\
\hline No & $6(15.4)$ & $12(30.8)$ & $21(53.8)$ & \\
\hline Antiplatelet & $10(20.0)$ & $14(28.0)$ & $26(52.0)$ & \\
\hline Anticoagulant & $2(11.1)$ & 7 (21.2) & $21(30.9)$ & \\
\hline Heparin bridge therapy ${ }^{a}$ & & & & .999 \\
\hline No & $0(0)$ & $2(28.6)$ & $5(71.4)$ & \\
\hline Yes & $2(8.7)$ & $5(21.7)$ & $16(69.6)$ & \\
\hline \multicolumn{5}{|l|}{ Lesion characteristics } \\
\hline Paris classification & & & & .507 \\
\hline Sessile & $9(19.1)$ & $11(23.4)$ & $27(57.4)$ & \\
\hline Flat & $9(12.5)$ & $22(30.6)$ & $41(56.9)$ & \\
\hline Size, $m m$, mean (SD) & $53.7(21.5)$ & $36.2(10.9)$ & $31.3(8.9)$ & $<.001^{d}$ \\
\hline Sigma & $0(0)$ & $0(0)$ & $1(100)$ & \\
\hline Descendent & $0(0)$ & $2(66.7)$ & $1(33.3)$ & \\
\hline Rectum & $1(16.7)$ & $1(16.7)$ & $4(66.7)$ & \\
\hline Proximal location ${ }^{b}$ (including TC) & & & & .893 \\
\hline No & $1(10.0)$ & $3(30.0)$ & $6(60.0)$ & \\
\hline Yes & $17(15.6)$ & $30(27.5)$ & $62(56.9)$ & \\
\hline Proximal location ${ }^{c}$ (excluding TC) & & & & .970 \\
\hline No & $4(13.8)$ & $8(27.6)$ & $17(58.6)$ & \\
\hline Yes & $14(15.6)$ & $25(27.8)$ & $51(56.7)$ & \\
\hline Histology & & & & .686 \\
\hline Adenoma & $9(12.5)$ & $19(26.4)$ & $44(61.1)$ & \\
\hline Adenoma-HGD & $8(22.9)$ & $11(31.4)$ & $16(45.7)$ & \\
\hline SI-ADC & $0(0)$ & $2(50)$ & $2(50)$ & \\
\hline Serrated & $0(0)$ & $1(25)$ & $3(75)$ & \\
\hline Serrated-HGD & $1(33.3)$ & $0(0)$ & $2(66.7)$ & \\
\hline SI-serrated & $0(0)$ & $0(0)$ & $1(100)$ & \\
\hline High-risk histology & & & & .236 \\
\hline No & 7 (10.3) & 19 (27.9) & $42(61.8)$ & \\
\hline Yes & $9(20.9)$ & $13(30.2)$ & $21(48.8)$ & \\
\hline
\end{tabular}


Supplementary Table 4. Continued

\begin{tabular}{|c|c|c|c|c|}
\hline Variable & $\begin{array}{l}\text { Failed closure, } \\
(\mathrm{n}=18[15.1 \%])\end{array}$ & $\begin{array}{l}\text { Partial closure, } \\
(\mathrm{n}=33[27.7 \%])\end{array}$ & $\begin{array}{l}\text { Complete closure, } \\
(\mathrm{n}=68[57.1 \%])\end{array}$ & $P$ value \\
\hline \multicolumn{5}{|l|}{ EMR technique } \\
\hline Time of procedure, min, mean (SD) & $68.3(28.0)$ & $40.0(19.2)$ & $35.9(22.5)$ & $<.001^{d}$ \\
\hline Difficulty & & & & $<.001^{d}$ \\
\hline Easy & $0(0)$ & $6(20.0)$ & $24(80.0)$ & \\
\hline Medium & $3(6.0)$ & $17(34.0)$ & $30(60.0)$ & \\
\hline Difficult & $15(39.5)$ & $10(26.3)$ & $13(34.2)$ & \\
\hline Accessibility & & & & $<.001^{d}$ \\
\hline Easy & $1(2.9)$ & $6(17.6)$ & $27(79.4)$ & \\
\hline Medium & 7 (11.5) & $19(31.1)$ & $35(57.4)$ & \\
\hline Difficult & $10(41.7)$ & 8 (33.3) & $6(25.0)$ & \\
\hline
\end{tabular}

NOTE. Values are $\mathrm{n}(\%)$ unless otherwise specified.

ADC, adenocarcinoma; ASA, American Society of Anesthesiologists; HGD, high-grade dysplasia; SI, superficial invasive; TC, transverse colon.

${ }^{a}$ Described only for patients who received the chronic anticoagulant treatment.

${ }^{b}$ Location from cecum to the TC (included).

${ }^{c}$ Location from cecum to the TC (excluded).

${ }^{d}$ Extremely significant $(P<.001)$.

eVery significant $(P<.01)$. 


\section{Prophylactic Clipping After Colorectal Endoscopic Resection Prevents Bleeding of Large, Proximal Polyps: Meta-analysis of Randomized Trials}

Marco Spadaccini, ${ }^{1,2, *}$ Eduardo Albéniz, ${ }^{3, *}$ Heiko Pohl, ${ }^{4}$ Roberta Maselli, ${ }^{1}$ Viveksandeep Thoguluva Chandrasekar, ${ }^{5}$ Loredana Correale, ${ }^{1}$ Andrea Anderloni, ${ }^{1}$ Silvia Carrara, ${ }^{1}$ Alessandro Fugazza, ${ }^{1}$ Matteo Badalamenti, ${ }^{2}$ Mineo Iwatate, ${ }^{6}$ Giulio Antonelli, ${ }^{7}$ Mónica Enguita-Germán, ${ }^{3}$ Marco Antonio Álvarez, ${ }^{8}$ Prateek Sharma, ${ }^{5}$ Douglas K. Rex, ${ }^{9}$ Cesare Hassan, ${ }^{7, \S}$ and Alessandro Repici ${ }^{1,2, \S}$

${ }^{1}$ Humanitas Research Hospital, Digestive Endoscopy Unit, Rozzano, Italy; ${ }^{2}$ Humanitas University, Department of Biomedical Sciences, Rozzano, Italy; ${ }^{3}$ Navarrabiomed Research Institute/Public University of Navarra/IdiSNA, Endoscopy Research Department, Pamplona, Spain; ${ }^{4}$ Dartmouth Geisel School of Medicine, Digestive Endoscopy Unit, Hanover, New Hampshire; ${ }^{5}$ Kansas City Veterans Affairs Medical Center, Gastroenterology and Hepatology, Kansas City, Missouri; ${ }^{6}$ Sano Hospital, Gastrointestinal Center and Institute of Minimally Invasive Endoscopic Care, Kobe, Japan; ${ }^{7}$ Hospital del Mar, Gastroenterology Department, Barcelona. Spain; ${ }^{8}$ Indiana University School of Medicine, Digestive Endoscopy Unit, Indianapolis, Indiana; ${ }^{9}$ Nuovo Regina Margherita Hospital, Digestive Endoscopy Unit, Rome, Italy

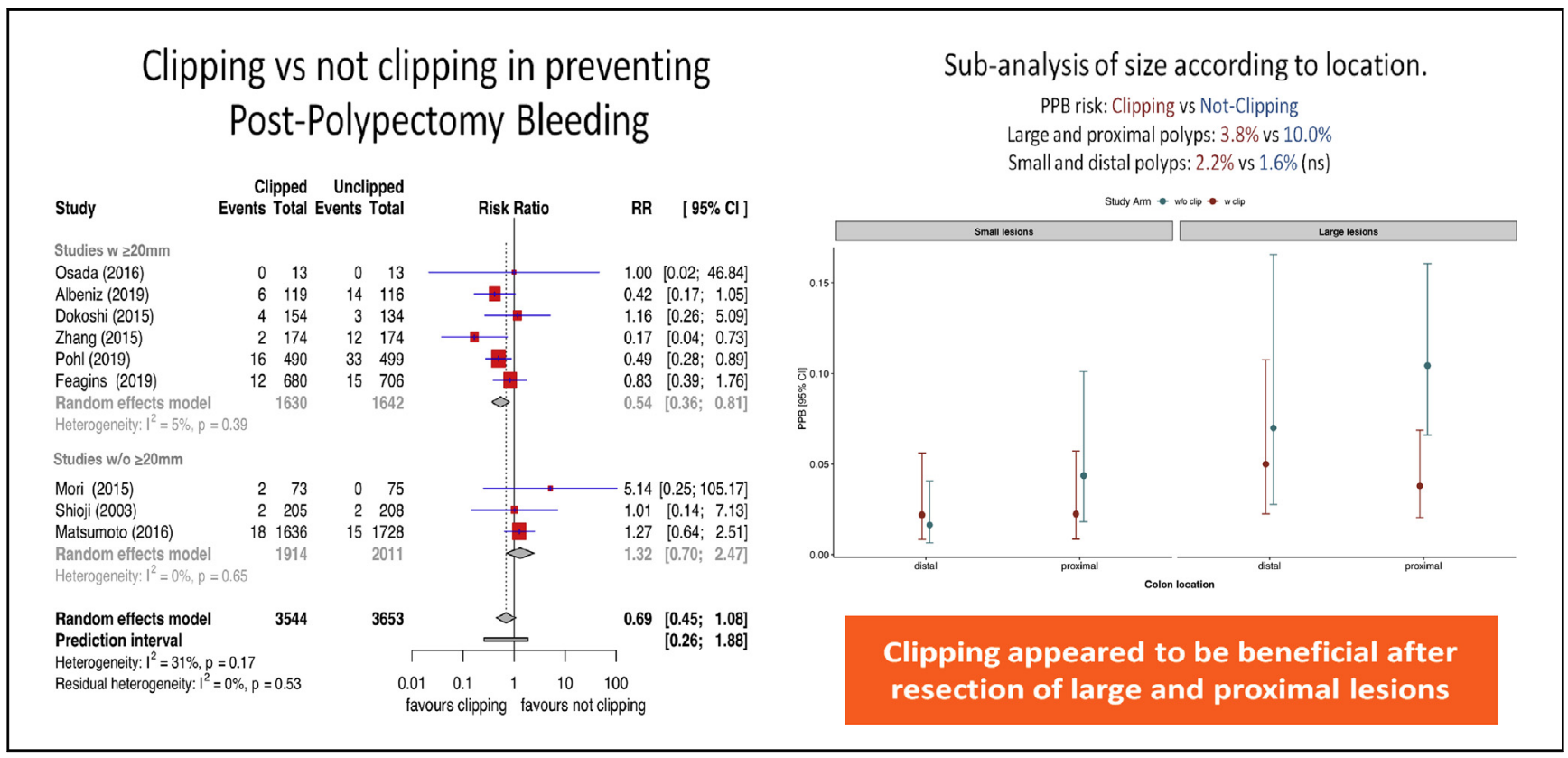

BACKGROUND \& AIMS: The benefits of prophylactic clipping to prevent bleeding after polypectomy are unclear. We conducted an updated meta-analysis of randomized trials to assess the efficacy of clipping in preventing bleeding after polypectomy, overall and according to polyp size and location. METHODS: We searched the MEDLINE/PubMed, Embase, and Scopus databases for randomized trials that compared the effects of clipping vs not clipping to prevent bleeding after polypectomy. We performed a random-effects meta-analysis to generate pooled relative risks (RRs) with 95\% CIs. Multilevel random-effects metaregression analysis was used to combine data on bleeding after polypectomy and estimate associations between rates of bleeding and polyp characteristics. RESULTS: We analyzed data from 9 trials, comprising 71897 colorectal lesions $(22.5 \% 20 \mathrm{~mm}$ or larger; $49.2 \%$ with proximal location). Clipping, compared with no clipping, did not significantly reduce the overall risk of postpolypectomy bleeding $(2.2 \%$ with clipping vs $3.3 \%$ with no clipping; RR, 0.69; 95\% confidence interval [CI], 0.45-1.08; $P=.072$ ). Clipping significantly reduced risk of bleeding after removal of polyps that were $20 \mathrm{~mm}$ or larger $(4.3 \% \mathrm{had}$ bleeding after clipping vs $7.6 \%$ had bleeding with no clipping; RR, $0.51 ; 95 \% \mathrm{CI}, 0.33-0.78 ; P=.020$ ) or that were in a proximal location $(3.0 \%$ had bleeding after clipping vs $6.2 \%$ had bleeding with no clipping; RR, 0.53; 95\% CI, 0.35-0.81; $P$ $<$.001). In multilevel metaregression analysis that adjusted 
for polyp size and location, prophylactic clipping was significantly associated with reduced risk of bleeding after removal of large proximal polyps (RR, $0.37 ; 95 \% \mathrm{CI}, 0.22-0.61$; $P=.021$ ) but not small proximal lesions (RR, 0.88 ; $95 \% \mathrm{CI}$, 0.48-1.62; $P=.581$ ). CONCLUSIONS: In a meta-analysis of randomized trials, we found that routine use of prophylactic clipping does not reduce risk of postpolypectomy bleeding overall. However, clipping appeared to reduce bleeding after removal of large (more than $20 \mathrm{~mm}$ ) proximal lesions.

Keywords: Colonoscopy; Comparison; Complication; PPB.

Colonoscopy and endoscopic resection of precancerous lesions significantly decreases the risk of colorectal cancer incidence and death. ${ }^{1-3}$ However, endoscopic procedures might result in adverse events, such as postprocedural pain, intraprocedural or postprocedural bleeding, perforation, and even death. ${ }^{4}$ Postprocedural (delayed) bleeding (PPB) after polypectomy and endoscopic mucosal resection is the most common major adverse event, ranging from $1 \%$ to $6 \%{ }^{5,6}$ Larger lesion size and proximal location are well-established risk factors for PPB., ${ }^{7,8}$ PPB may require the need for hospitalization, blood transfusion, and further endoscopic or more invasive treatments, representing a risk for the patient and a burden to the health system.

Despite a lack of high-quality evidence, prophylactic clipping has been advocated as a technique to reduce the risk of PPB. For instance, the European Society of Gastrointestinal Endoscopy suggests that there may be a role for prophylactic clipping and that this decision be based on patient risk factors. ${ }^{4}$ However, this recommendation has been graded as weak because it is based on low-quality evidence. Previous meta-analyses, mostly analyzing studies with small lesions $(<20 \mathrm{~mm})$ and at high risk of bias, reported no protective effect of prophylactic clipping for nonpedunculated lesions. ${ }^{9-11}$ Recently, high-quality randomized controlled trials (RCTs) have been published investigating the efficacy of prophylactic clipping primarily for lesions larger than 20 $\mathrm{mm}$. However, there is uncertainty on the overall efficacy of clipping and whether it is possible to identify subgroups that may benefit from prophylactic clipping. This issue is clinically relevant, considering the costs and technical complexity of clipping.

We performed a systematic review and meta-analysis of all available RCTs to clarify the role of prophylactic clipping in preventing PPB after endoscopic resection of colorectal lesions.

\section{Methods}

The methods of our analysis and inclusion criteria were based on Preferred Reporting Items for Systematic Reviews and Meta-Analyses (PRISMA) recommendations. ${ }^{12}$ Our systematic review protocol was registered with the International Prospective Register of Systematic Reviews (PROSPERO) in October 2019.

\section{WHAT YOU NEED TO KNOW}

\section{BACKGROUND AND CONTEXT}

It is not clear whether prophylactic clipping prevents bleeding after polypectomy.

\section{NEW FINDINGS}

A meta-analysis of randomized trials showed that routine use of prophylactic clipping does not reduce risk of postpolypectomy bleeding, overall. However, clipping appeared to reduce bleeding after removal of large (more than $20 \mathrm{~mm}$ ) and proximal lesions.

\section{LIMITATIONS}

This was a meta-analysis of previous studies. Further prospective studies are needed.

\section{IMPACT}

Only large (more than $20 \mathrm{~mm}$ ) and proximal colorectal lesions should be clipped to prevent bleeding after polypectomy.

The following methods are reported in the Supplementary Materials: data sources and search strategy, selection process, data extraction, and quality assessment.

\section{Inclusion and Exclusion Criteria}

For the purpose of our meta-analysis, we screened all clinical studies for the following inclusion criteria:

- Population: all adults undergoing endoscopic resection of colorectal lesions.

- Intervention: postpolypectomy prophylactic closure of mucosal defects with hemoclips.

- Comparison: no prophylactic clipping postpolypectomy.

- Outcome: risk of delayed PPB.

- Study design: only RCTs were considered.

Exclusion criteria were as follows:

- Essential information not available.

- Studies not published as full text article.

- Studies not published in English.

- Studies considering as a comparator any intervention strategy for PPB prophylaxis (ie, cauterization of postprocedural ulcer floor by argon plasma coagulation, injective strategies, use of topical hemostatic agents, etc).

- Studies including fewer than 10 patients in each group.

* Authors share co-first authorship; ${ }^{\S}$ Authors share co-senior authorship.

Abbreviations used in this paper: OR, odds ratio; PI, predication interval; PPB, postpolypectomy (delayed) bleeding; RCT, randomized controlled trial; $\mathbf{R R}$, risk ratio.

\section{Most current article}

(C) 2020 by the AGA Institute 0016-5085/\$36.00

https://doi.org/10.1053/j.gastro.2020.03.051 


\section{Study Endpoints}

The primary aim of this study was to assess the efficacy of endoscopic clipping for the prevention of PPB. We included all patients randomly assigned to the clipping group in the intention to treat analysis. When both sets of data were provided, values from intention to treat were preferred to per protocol. A per protocol analysis was also conducted including only those patients who underwent a complete closure of the mucosal defect. $P P B$ was defined as any postprocedural clinically evident hematochezia that required medical intervention (hospitalization, blood transfusion, repeated colonoscopy, angiography, or surgery) or caused a more than $2-\mathrm{g} / \mathrm{dL}$ decrease in blood hemoglobin concentration.

The secondary aim of the study was to determine the effect of risk factors (patient and lesion) on the risk of PPB with and without clipping. The risk factors taken into account were patient age and sex, use of antiplatelet/anticoagulant therapy, and lesion characteristics such as the polyp size, morphology and location (proximal), and histology (serrated and adenomatous). Lesions $\geq 20 \mathrm{~mm}$ were defined as large, and morphology was described according to the Paris classification. ${ }^{13}$ Lesion location was defined as per the reporting in the studies. An additional secondary aim was to assess the efficacy of endoscopic clipping for the prevention of other postprocedural adverse events such as perforation.

\section{Statistical Analysis}

All statistical analyses were conducted with the $\mathrm{R}$, version 3.5.1 (R Core Team, Vienna, Austria). ${ }^{14}$ In particular, we used the meta ${ }^{15}$ and metafor ${ }^{16}$ libraries in $\mathrm{R}$ to conduct the metaanalysis and metaregression analysis. All tests were 2 tailed. For all tests, a probability level less than .05 was considered significant. Study characteristics were summarized by using descriptive statistics. Risk ratios (RRs) and 95\% confidence intervals (CIs) were estimated with the Mantel-Haenszel (fixed-effects model) and the DerSimonian-Laird (random-effects model) methods. The summary effect sizes from randomeffects meta-analysis (the assumption that the effects estimated in the different studies were not identical) were calculated. We assessed statistical heterogeneity between studies by visual inspection of forest plots and by the $I^{2}$ and $\chi^{2}$ statistics for heterogeneity. $I^{2}$ values of $0 \%-30 \%, 30 \%-60 \%$, $50 \%-90 \%$, and $75 \%-100 \%$ were classified as low, moderate, substantial, and considerable heterogeneity, respectively. We also calculated the $95 \%$ CIs for pooled estimates and the prediction interval (PI). ${ }^{17}$

Sensitivity analyses were performed to assess the stability of the results; namely, a single study in this meta-analysis was omitted one at a time to assess the influence of that individual study on the pooled RR. ${ }^{18}$ Visual inspection of funnel plot asymmetry was conducted along with the Egger's weighted regression method to assess publication bias. $P<.05$ was considered statistically significant.

Metaregression Analysis of Relative Risk Estimates and Subgroup Analyses. We conducted a metaregression analysis to investigate the impact of various risk factors on the study estimates of relative risk. The natural logarithm of the risk ratio was the dependent variable, and study level characteristics (eg, number of participants, mean age, percentage of male patients, percentage of proximal polyps, percentage of large polyps) were entered as explanatory factors. As the first step, we performed univariate regression analyses for each factor. All significant factors (at a significance level of $P<.10$ ) were then included in a multivariable regression model. The estimated coefficients of the regression model corresponded to differences in the log risk ratios for 1 unit of difference in the explanatory factor (continuous variable) or for each category relative to the baseline category (for categorical explanatory variables). Of note, input percentages (eg, the percentage of proximal polyps) were analyzed as continuous variables, expressed originally as decimal fractions. The percentages were multiplied by 10 before entering in the regression model. Therefore, a change of 1 unit on the scaled variables corresponds to a change of 0.10 $(10 \%)$ in the original variables. Subgroup analyses were also performed according to the polyp location (proximal vs distal colon) and size (large vs small polyps).

Multilevel (Random Effects) Model: Meta-analysis of Rates of Postpolypectomy Bleeding With Clipping vs No Clipping. As a subsequent step, we performed a multilevel regression analysis to estimate the rates of PPB associated with clipping vs no clipping. The outcome measure in this analysis was the proportion of PPB transformed via logits. Because the studies included in the analysis reported multiple outcome measures, we used a multilevel meta-analysis method to combine data, with random effects at both the study level and at the outcome level (see Supplementary Materials). The multilevel model was extended by including predictor variables (ie, study group, prevalence of large lesions, and proximal colon location) in an attempt to determine variables that moderate the effect. In this model, predictor variables were aggregated at the study-arm level. We also considered appropriate interactions between these variables. Data were presented as odds ratios (ORs) and 95\% CIs. This analysis was an indirect way to deal with aspects such as the possibility of effect modification by polyp characteristics and to examine for decreasing bleeding risk with clipping vs not clipping.

\section{Results}

\section{Study Characteristics and Quality}

The initial literature search resulted in 1112 articles (Figure 1). A total of 9 trials were included in the final analysis. ${ }^{19-27}$ Study characteristics are shown in Table 1. Studies were published between 2003 and 2019 in the United States $(n=3)$, Europe $(n=1)$, and Asia $(n=5)$. Five studies involved multiple centers, and 4 studies were singlecenter experiences. The objective assessment of the risk of bias is reported in Supplementary Table 1.

The total number of participants included in the intention to treat analysis was 4557 (2288 in the clipping and 2269 in the control group), and the individual study sample size ranged from 26 to 1499 patients. The mean patient age ranged from 60.5 to 72.7 years. A total of 7197 colorectal lesions were analyzed (3544 clip and 3653 control group). The percentage of proximal polyps ranged from $25 \%$ to $92 \%$, with a mean percentage of $49.2 \%$. The mean size of polyps ranged from $7.8 \mathrm{~mm}$ to $37.3 \mathrm{~mm}$, with an average polyp size of $18.6 \mathrm{~mm}$. Of the analyzed studies, 3 included only patients with polyps $>20 \mathrm{~mm}$; 3 involved patients having polyps of any size (mean percentage of lesions $\geq 20$ 


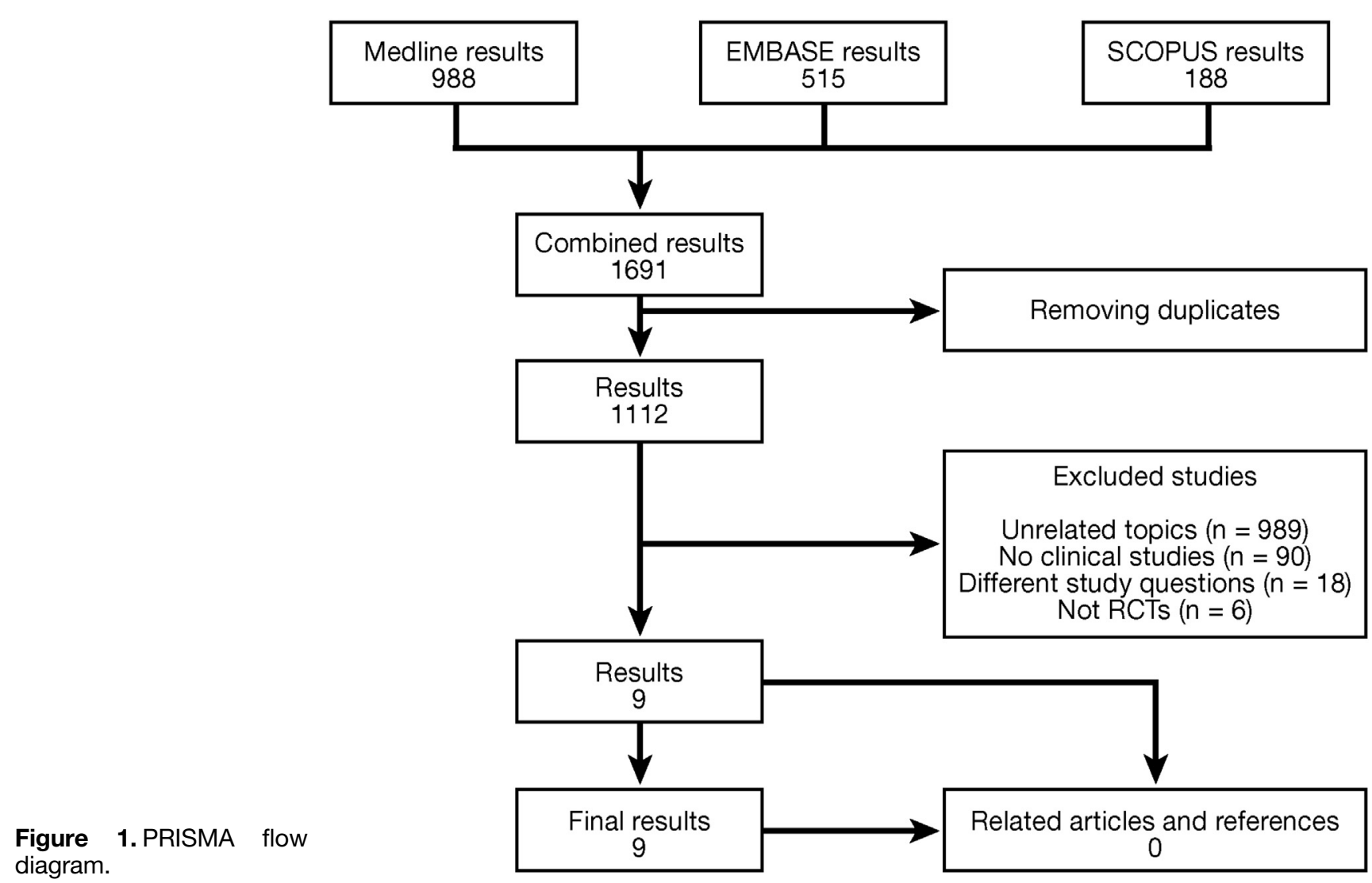

$\mathrm{mm}, 19.4 \%$ ), whereas the remaining 3 studies included only lesions $<20 \mathrm{~mm}$ in size.

\section{Effect of Clipping on Postpolypectomy Bleeding Risk}

Based on data reported by all 9 trials, the estimates of PPB risk between the control and clip groups were 3.3\% (95\% CI, 1.9-5.7) and 2.2\% (95\% CI, 1.2-3.9) (multilevel random-effect model accounting for the underlying heterogeneity between and within trials), respectively (Supplementary Figure 1). No significant difference between the 2 groups was noted for the risk of PPB when a randomeffects model was applied (RR, 0.69; 95\% CI, 0.45-1.08; $P=$ .072) (Figure 2). Moderate heterogeneity among the studies existed $\left(I^{2}=31 \% ; P=.170\right)$, and the likelihood of publication bias was low $(P=.42)$ (Supplementary Figure 2). The results of the meta-analysis did not change substantially when data from per protocol analysis were combined (Supplementary Figure 3). The results of the leave-one-out sensitivity analysis are reported in Supplementary Table 2. Only 1 study that evaluated patients with lesions $<20 \mathrm{~mm}$ was determined to have a substantial influence on the overall effect size. ${ }^{23}$ After removing this trial (in a sensitivity analysis), the heterogeneity was significantly reduced $\left(I^{2}=\right.$ $8.2 \%$ ), and the pooled (random effects) RR was 0.58 (95\% CI, 0.39-0.88). To further investigate the impact of studylevel characteristics on RR estimates, we performed a metaregression analysis (Table 2). The analysis showed that use of prophylactic clipping was associated with a significant reduction in PPB as the percentage of large lesions in the study population increased. The meta-regression coefficient for the percentage of large $(\geq 20 \mathrm{~mm})$ lesions was 0.92 (95\% CI, 0.85-0.98), indicating that for every $10 \%$ increase in the percentage of large lesions, the RR of PPB decreased by $8 \%(95 \% \mathrm{CI}, 2-15)$ if prophylactic clipping was used (Supplementary Figure 4). This finding was remarkably robust in the multilevel multivariate analysis (Supplementary Table 3).

\section{Effect of Clipping on Postpolypectomy Bleeding Risk According to Polyp Size}

A subgroup analysis by polyp size (large vs small polyps) confirmed the described meta-regression result (Figure 3). A beneficial effect of clipping was determined among studies including $\geq 20$-mm polyps (RR, $0.51 ; 95 \% \mathrm{CI}$, 0.33 0.78 ), whereas among those with $<20$-mm polyps, there was no significant benefit (RR, 1.04; 95\% CI, 0.60-1.79). According to multilevel random-effects meta-regression analysis, the estimated rates of PPB among $\geq 20$-mm and $<20$-mm lesions were $7.6 \%(95 \% \mathrm{CI}, 4.9-11.5)$ and $1.8 \%$ (95\% CI, 1.1-2.9), respectively, in the control group compared with $4.3 \%$ (95\% CI, 2.5-7.1) and 1.4\% (95\% CI, 0.9-2.4), respectively, in the prophylactic clipping group (Supplementary Table 4 and Supplementary Figure 5). Again, the multilevel model indicated showed a protective effect of clipping for large polyps that remained significant 
Table 1.Study Characteristics

\begin{tabular}{|c|c|c|c|c|c|c|c|c|c|c|}
\hline Trial & Country & $\begin{array}{l}\text { Randomization } \\
\text { level (patients } \\
\text { /polyps) }\end{array}$ & $\begin{array}{l}\text { Randomization } \\
\text { time (pre-/ } \\
\text { postresection) }\end{array}$ & $\begin{array}{c}\text { Patients } \\
\text { (clipped/ } \\
\text { unclipped), n }\end{array}$ & $\begin{array}{c}\text { Male } \\
\text { (clipped/ } \\
\text { unclipped), n }\end{array}$ & $\begin{array}{l}\text { Mean age } \\
\text { (clipped/ } \\
\text { unclipped), y }\end{array}$ & $\begin{array}{l}\text { Polyps (clipped/ } \\
\text { unclipped), n }\end{array}$ & $\begin{array}{l}\text { Inclusion criteria } \\
\text { for polyp size }\end{array}$ & $\begin{array}{l}\text { Number of large } \\
\text { lesions (clipped } \\
\text { /unclipped) }\end{array}$ & $\begin{array}{c}\text { Number of } \\
\text { proximal } \\
\text { lesions (clipped/ } \\
\text { unclipped) }\end{array}$ \\
\hline $\begin{array}{l}\text { Albéniz et } \mathrm{al}^{25} \\
\text { (2019) }\end{array}$ & Spain & Patients & Post & $235(119 / 116)$ & $158(77 / 81)$ & $71.9(72.7 / 71.1)$ & $235(119 / 116)$ & $\geq 20 \mathrm{~mm}^{a}$ & $235(119 / 116)$ & $213(109 / 104)$ \\
\hline $\begin{array}{l}\text { Dokoshi et } \mathrm{al}^{20} \\
\text { (2015) }\end{array}$ & Japan & Patients & Pre & $157(89 / 68)$ & NA & $67.4(67.1 / 67.8)$ & $288(154 / 134)$ & $\begin{array}{c}\text { Polyps of any size } \\
(5 \%, \geq 20 \mathrm{~mm})\end{array}$ & $14(8 / 6)$ & $74(40 / 34)$ \\
\hline $\begin{array}{l}\text { Feagins et } \mathrm{al}^{27} \\
(2019)\end{array}$ & $\begin{array}{l}\text { United } \\
\text { States }\end{array}$ & Patients & Post & $1050(530 / 520)$ & $1015(516 / 499)$ & $64.25(64.5 / 64)$ & $1386(680 / 706)$ & $\begin{array}{c}\text { Polyps of any size } \\
(39 \%, \geq 20 \mathrm{~mm})\end{array}$ & $222(101 / 121)$ & $536(261 / 275)$ \\
\hline $\begin{array}{l}\text { Matsumoto et } \\
\mathrm{al}^{23}(2016)\end{array}$ & Japan & Patients & Pre & $1499(752 / 747)$ & $1047(534 / 513)$ & $60.75(60.5 / 61)$ & $3364(1636 / 1728)$ & $<20 \mathrm{~mm}$ & $0(0 / 0)$ & $1668(823 / 845)$ \\
\hline $\begin{array}{l}\text { Mori et } \mathrm{al}^{21} \\
(2015)\end{array}$ & Japan & Polyps & Pre & NA & NA & NA & $148(73 / 75)$ & $<20 \mathrm{~mm}^{a}$ & $0(0 / 0)$ & $42(21 / 21)$ \\
\hline $\begin{array}{l}\text { Osada et } \mathrm{al}^{24} \\
\quad(2016)\end{array}$ & Japan & Patients & NA & $26(13 / 13)$ & $13(9 / 4)$ & $67.5(68.8 / 66.2)$ & $26(13 / 13)$ & $\geq 20 \mathrm{~mm}$ & $26(13 / 13)$ & $18(8 / 10)$ \\
\hline $\begin{array}{l}\text { Pohl et al }{ }^{26} \\
(2019)\end{array}$ & $\begin{array}{l}\text { United } \\
\text { States }\end{array}$ & Patients & Pre & $919(455 / 464)$ & 547 (265/282) & $65.1(65.1 / 65.1)$ & $989(490 / 499)$ & $\geq 20 \mathrm{~mm}^{a}$ & 989 (490/499) & $658(327 / 331)$ \\
\hline $\begin{array}{c}\text { Shioji et } \text { al }^{19} \\
(2003)\end{array}$ & Japan & Polyps & Post & $323(156 / 167)$ & $248(118 / 130)$ & $63.5(64 / 63)$ & $413(205 / 208)$ & $<20 \mathrm{~mm}$ & $0(0 / 0)$ & $187(97 / 90)$ \\
\hline $\begin{array}{l}\text { Zhang et } \mathrm{al}^{22} \\
\text { (2015) }\end{array}$ & China & Patients & NA & $348(174 / 174)$ & $219(112 / 107)$ & $66.05(67.9 / 64.2)$ & $348(174 / 174)$ & $\begin{array}{l}\text { Polyps of any size } \\
(36 \%, \geq 20 \mathrm{~mm})\end{array}$ & $130(63 / 67)$ & $101(50 / 51)$ \\
\hline
\end{tabular}

NA, not available.

aStandardized methods to measure polyp size were used. 


\section{Study \\ Clipped Unclipped \\ Events Total Events Total}

Studies $w \geq 20 \mathrm{~mm}$

Osada (2016)

Albeniz (2019)

Dokoshi (2015)

Zhang (2015)

Pohl (2019)

Feagins (2019)

Random effects model

Heterogeneity: $\left.\right|^{2}=5 \%, P=.39$

Studies w/o $\geq 20 \mathrm{~mm}$

Mori (2015)

Shioji (2003)

Matsumoto (2016)

Random effects model

Heterogeneity: $I^{2}=0 \%, P=.65$

$\begin{array}{rr}0 & 13 \\ 6 & 119 \\ 4 & 154 \\ 2 & 174 \\ 16 & 490 \\ 12 & 680 \\ & 1630\end{array}$

$\begin{array}{rr}0 & 13 \\ 14 & 116 \\ 3 & 134 \\ 12 & 174 \\ 33 & 499 \\ 15 & 706 \\ & 1642\end{array}$

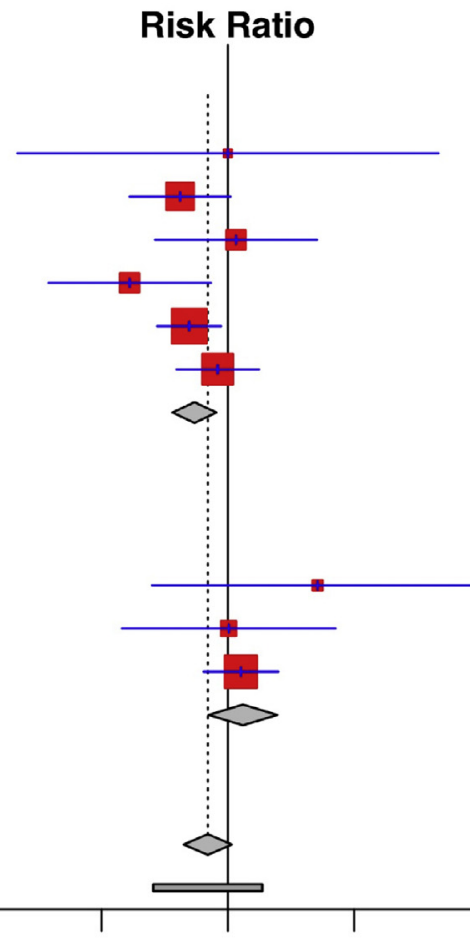

RR [ $95 \% \mathrm{Cl}]$

$1.00 \quad[0.02 ; 46.84]$

$0.42 \quad[0.17 ; 1.05]$

$1.16 \quad[0.26 ; 5.09]$

$0.17 \quad[0.04 ; 0.73]$

$0.49 \quad[0.28 ; 0.89]$

$0.83 \quad[0.39 ; 1.76]$

$0.54 \quad[0.36 ; 0.81]$

$5.14[0.25 ; 105.17]$

$1.01 \quad[0.14 ; 7.13]$

$1.27 \quad[0.64 ; 2.51]$

$1.32 \quad[0.70 ; 2.47]$

$0.69[0.45 ; 1.08]$ $[0.26 ; 1.88]$
Random effects model

Prediction interval

Heterogeneity: $\mathrm{I}^{2}=31 \%, P=.17$

Residual heterogeneity: $I^{2}=0 \%, P=.53$
3544

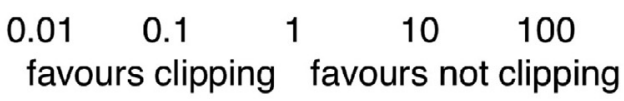

Figure 2. Forest plot comparing the clipping vs not clipping arms. The relative risk of developing a PPB event for polyps clipped or unclipped is displayed. The line of no effect (vertical line) separates outcomes that favor clipping and not clipping. The red boxes represent the point estimates, and the horizontal lines represent the associated 95\% Cls for each study. The area of each square is proportional to the study's weight in the meta-analysis. Overlapping Cls of the individual studies and an $I^{2}$ value of $31 \%$ with a nonsignificant $P$ value $(0.17)$ indicates homogeneity of the studies. Studies were separated according to size criteria for polyp inclusion: studies including large lesions in their data sets (ie, with lesions $\geq 20 \mathrm{~mm}$ ) and those including only lesions $<20 \mathrm{~mm}$ (ie, without lesions $\geq 20 \mathrm{~mm}$ ).

after adjusting for the prevalence of proximal polyps (OR, $0.53 ; 95 \% \mathrm{CI}, 0.31-0.90)$.

\section{Effect of Clipping on Postpolypectomy Bleeding Risk Based on Polyp Location With Subanalysis by Size}

Seven trials with available data on proximal/distal lesions (1618/1648 clipped and 1650/1720 control groups) were included in this analysis. Figure 4 graphs the risk ratios and 95\% CIs from the individual trials and the pooled results. Among proximally located lesions, there was a significant association between use of prophylactic clipping and risk of PPB (RR, 0.53; 95\% CI, 0.35-0.81). However, among distally located lesions, there was no significant difference between the 2 groups and risk of PPB (RR, 1.01; 95\% CI, 0.43-2.37). This association was corroborated by both sensitivity analysis among studies defining proximal lesions as those located proximal to the splenic flexure (including transverse colon) and those defining proximal lesions as in the cecum, ascending colon, or hepatic flexure (Supplementary Figures 6 and 7). According to a multilevel random effects metaregression analysis, the estimated rates of PPB in the distal and proximal colon were $2.7 \%$ (95\% CI, 1.3-5.4; PI, 0.5-12.3) and $6.2 \%$ (95\% CI, 3.4-11.1; PI, 1.3-24.3), respectively, in the control group compared to $3.3 \%$ (95\% CI, 1.6-6.5; PI, $0.7-14.6)$ and $3.0 \%(95 \% \mathrm{CI}, 1.5-5.7 ; \mathrm{PI}, 0.6-13.2)$, respectively, in the clip group (Supplementary Figure 8). The described model showed a protective effect of clipping for proximally located polyps (clipped proximal vs unclipped proximal polyps: OR, 0.46; 95\% CI, 0.24-0.88) (Supplementary Table 5).

After adjusting for the prevalence of large lesions in the multilevel model, the benefit of clipping in reducing PPB was significant only for large proximal lesions (clipped vs unclipped polyps: OR, 0.37; 95\% CI, 0.22-0.61; $P=.021$ ) but not for small proximal lesions (clipped vs unclipped polyps: OR, 0.88 ; $95 \% \mathrm{CI}, 0.48-1.62 ; P=.581$ ). This finding was remarkably robust in subgroup and sensitivity analyses (Figure 5 and Supplementary Figure 9). Clipping was also not beneficial for large distal lesions (RR, $0.70 ; 95 \% \mathrm{CI}$, $0.22-2.27$ ), although this outcome mainly depended on the lack of benefit in only 1 large series (Supplementary Figure 10), or for small distal lesions (RR, 1.34; 95\% CI, 0.42-4.35) (Figure 5). 
Table 2. Meta-analysis of Overall Risk of PPB-Metaregression Results

\begin{tabular}{|c|c|c|c|c|c|c|}
\hline \multirow[b]{2}{*}{ Variable } & \multicolumn{3}{|c|}{ Univariate Metaregression analysis } & \multicolumn{3}{|c|}{ Multivariate Metaregression analysis } \\
\hline & $\mathrm{RR}$ & $95 \% \mathrm{Cl}$ & $P$ value & $\mathrm{RR}$ & $95 \% \mathrm{Cl}$ & $P$ value \\
\hline Number of participants (per 100-individual increase) & 1.03 & $1.00-1.06$ & .051 & 1.01 & $0.98-1.05$ & .438 \\
\hline \multicolumn{7}{|l|}{ Mean patient age, $y$} \\
\hline$<66$ & 1 & & & & & \\
\hline$>65$ & 0.54 & $0.23-1.26$ & .155 & & & \\
\hline \multicolumn{7}{|l|}{ Region } \\
\hline United States/Europe & 1 & & & & & \\
\hline Asia & 0.99 & $0.33-3.00$ & .991 & & & \\
\hline Percentage of male participants & 0.99 & $0.40-0.33$ & .957 & & & \\
\hline Percentage of large lesions & 0.92 & $0.85-0.98$ & .017 & 0.92 & $0.86-0.99$ & .042 \\
\hline Percentage of proximal lesions & 0.88 & $0.74-1.05$ & .152 & & & \\
\hline Percentage of nonpolypoid lesions & 1.27 & $0.21-7.54$ & .792 & & & \\
\hline Percentage of nonpedunculated lesions & 0.14 & $0.01-4.21$ & .256 & & & \\
\hline Percentage of adenomatous lesions & 1.10 & $0.78-1.52$ & .680 & & & \\
\hline Percentage of serrated lesions & 0.11 & $0.00-5.72$ & .276 & & & \\
\hline Percentage of patients with antiplatelet therapy & 0.02 & $0.00-3.78$ & .144 & & & \\
\hline
\end{tabular}

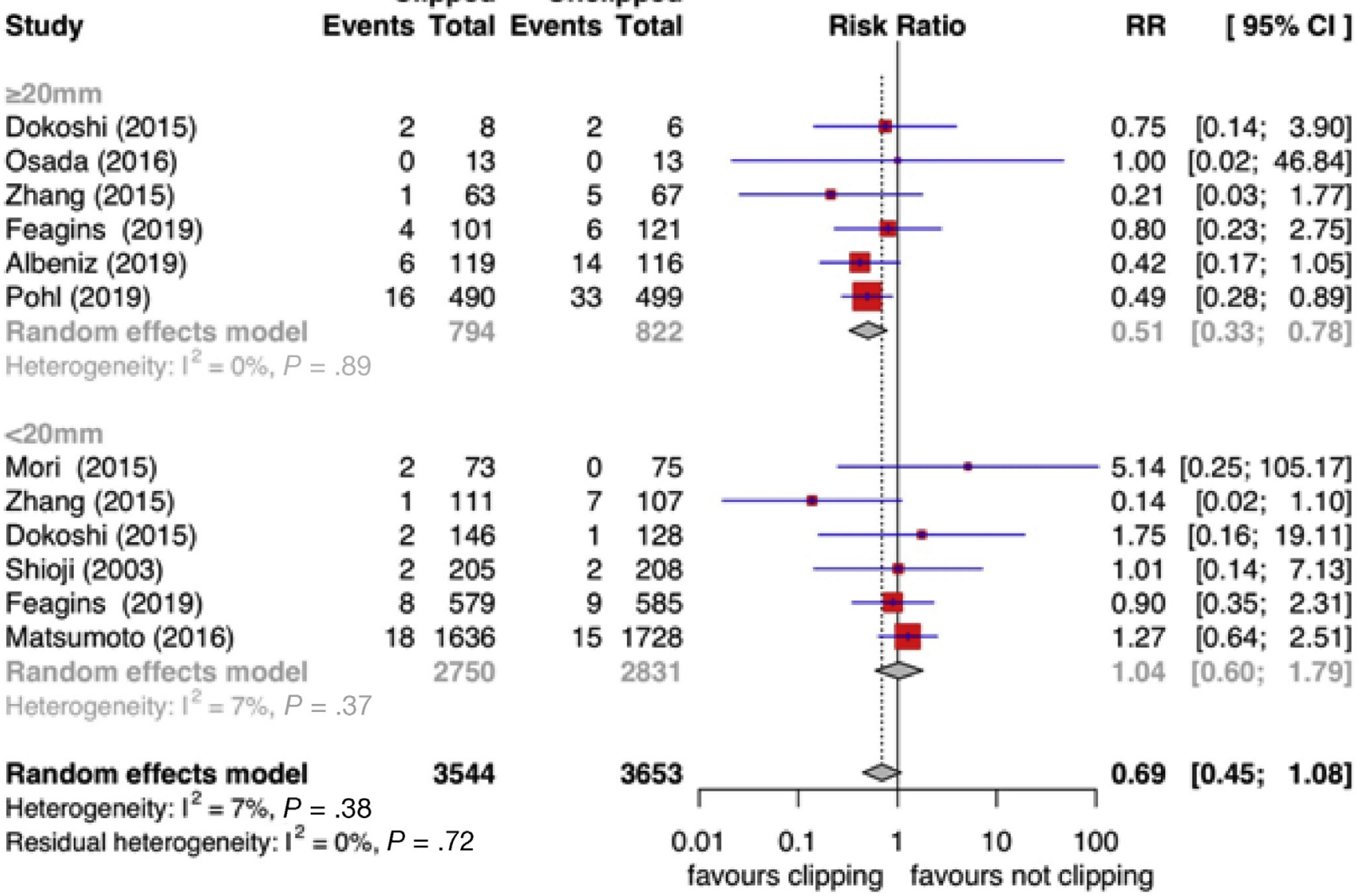

Figure 3. Subgroup analysis of relative risk of developing PPB events with and without clipping for large vs small polyps. For each subgroup, the relative risk of developing PPB for polyps clipped or unclipped is displayed. The line of no effect (vertical line) separates outcomes that favor clipping and not clipping. The red boxes represent the point estimates, and the horizontal lines represent the associated 95\% confidence intervals for each study. The area of each square is proportional to the study's weight in the meta-analysis. Overlapping confidence intervals of the individual studies and $I^{2}$ values with nonsignificant $P$ values indicate homogeneity of the studies. 


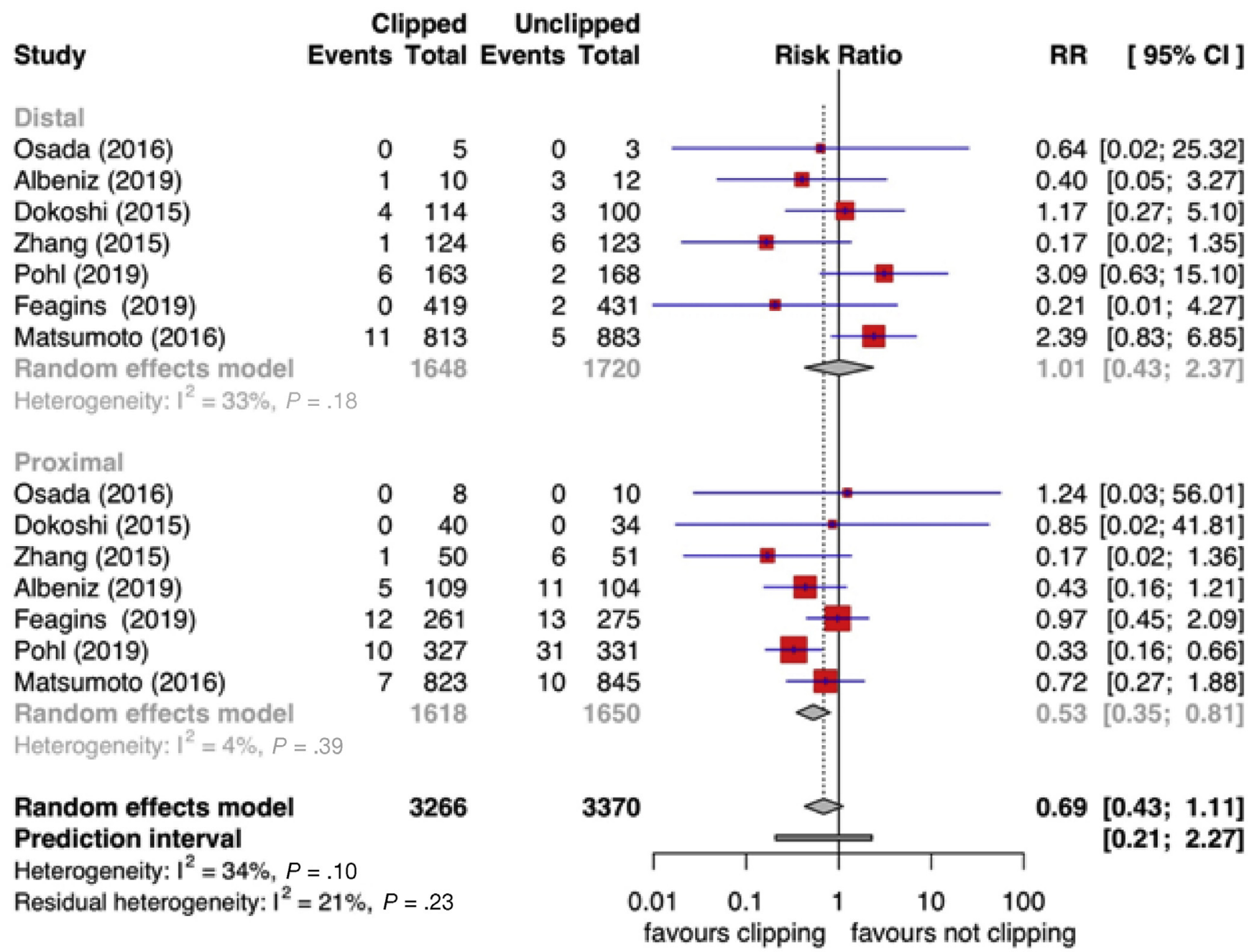

Figure 4. Subgroup analysis comparing the clipping vs not clipping arms for successful control of PPB events for distally and proximally located lesions. For each subgroup, the relative risk of developing a PPB for polyps clipped or unclipped is displayed. The line of no effect (vertical line) separates outcomes that favor clipping and not clipping. The red boxes represent the point estimates, and the horizontal lines represent the associated $95 \%$ Cls for each study. The area of each square is proportional to the study's weight in the meta-analysis. Overlapping Cls of the individual studies and a $l^{2}$ value with a nonsignificant $P$ value indicates homogeneity of the studies.

\section{Perforations}

Data on perforation were available in 6 studies. In total, 14 perforation events were reported $(6 / 1074,0.56 \%$ in the clip group and $8 / 1085,0.74 \%$ in control group), with a RR of 0.70 (95\% CI, $0.25-1.91 ; P=.480 ; I^{2}=0.0 \%$ ), indicating no significant differences between groups.

\section{Additional Analysis}

The quality of evidence was assessed by applying the GRADE methodology. The level of evidence for RCTs was downgraded due to low-moderate quality of the included RCTs (assessed by Cochrane risk bias tool for randomized studies) and the inconsistency owing to heterogeneity among patients (ie, different indications for resection in terms of lesion size or location). Details can be found in Supplementary Table 6

Additional technical features such as types of clips used and electrosurgical unit characteristics are detailed in Supplementary Table 7.

\section{Discussion}

According to our meta-analysis, routine practice of endoscopic clipping as a prophylactic intervention does not reduce the risk of PPB. However, clipping was effective in reducing the risk of PPB by nearly $50 \%$ for large lesions $(\geq 20 \mathrm{~mm})$. If such lesions do not undergo endoscopic clipping, there was a 4-fold increase in the baseline risk of PPB as compared with those $<20 \mathrm{~mm}$. Such benefit appeared to be limited to large lesions located in the proximal colon.

The results of our analysis are relevant for several reasons. Meta-analysis of randomized trials is considered as the strongest evidence for clinical recommendations. The lack of efficacy of prophylactic clipping after any polypectomy was confirmed by our results showing no significant difference in PPB risk between the 2 groups (clip group vs control group). This was further confirmed by the leave-one-out analysis-that is, clipping became significantly effective when excluding this series-because this was the largest 


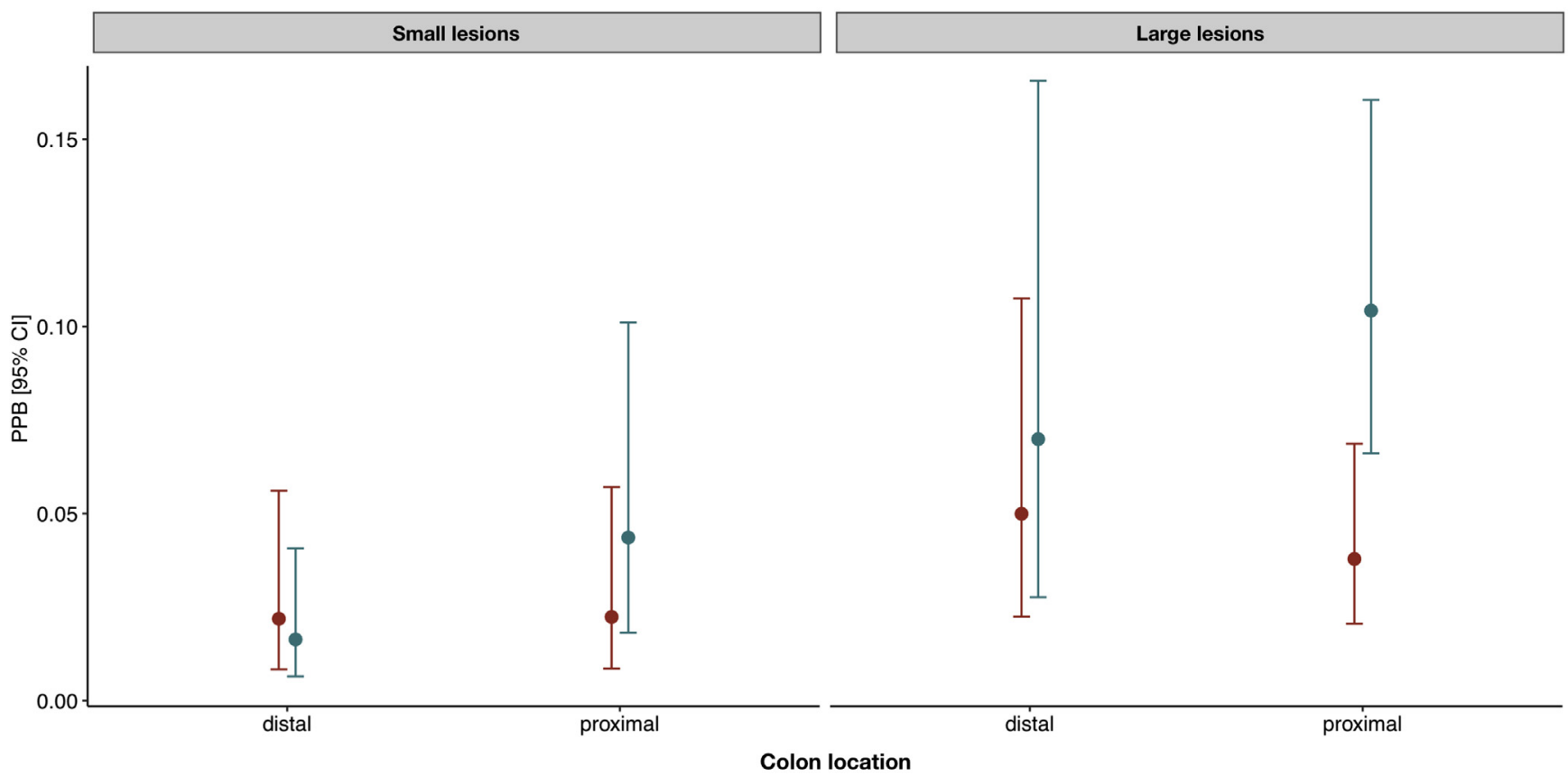

Figure 5. Subanalysis of size according to location. In this analysis, results from the studies were analyzed according 2 subgroups: studies including more than $50 \%$ of large lesions in their analysis sets were considered large polyps, and the others were considered small polyps. The PPB risks for clipped and unclipped large and proximal polyps were 3.8\% (95\% $\mathrm{Cl}$, 2.1-6.9) and $10.0 \%(95 \% \mathrm{Cl}, 6.6-16.7)(\mathrm{RR}, 0.34 ; 95 \% \mathrm{Cl}, 0.19-0.65)$, whereas PPB risks for small and proximal polyps were $2.2 \%(95 \% \mathrm{Cl}, 0.9-5.7)$ and $4.3 \%(95 \% \mathrm{Cl}, 1.1-10.1)$, respectively (RR, $0.50 ; 95 \% \mathrm{Cl}, 0.16-1.57)$. The corresponding features for clipped and unclipped large and distal polyps were 5.0\% $(95 \% \mathrm{Cl}, 2.2-10.7)$ and $7.0 \%(2.8-16.6)$, respectively (RR, 0.70; $95 \% \mathrm{Cl}, 0.22-2.27)$, whereas PPB risks for small and distal polyps were $2.2 \%(95 \% \mathrm{Cl}, 0.8-5.6)$ and $1.6 \%(95 \% \mathrm{Cl}, 0.7-4.1)$, respectively (RR, $1.34 ; 95 \% \mathrm{Cl}, 0.42-4.35)$.

series on small lesions. This inference is a distinct advantage of our analysis, because previous systematic reviews were not powered enough to perform it. ${ }^{9-11,30-32}$

Our analysis showed the clinical relevance of 2 main factors: polyp size and location, on both the absolute risk of PPB and the relative efficacy of clipping, as well as their hierarchical interaction. Our adjusted estimates attributed a nearly 2 -fold increase-from $4.3 \%$ to $7.6 \%$-in the risk of PPB for lesions $\geq 20 \mathrm{~mm}$. This finding was supported by 2 additional results. First, the PPB risk estimate proportionally increased as the percentage of $\geq 20 \mathrm{~mm}$ increased in the included studies. Second, this cutoff size was the only significant risk factor associated at metaregression with PPB risk.

Although showing an increase in the PPB risk according to size, our data also showed a clear reduction in PPB risk with clipping. A 50\% reduction in PPB for those with clipped $\geq 20 \mathrm{~mm}$ lesions directly translated to a number needed to treat of 23 for lesions to be clipped to prevent 1 additional bleeding. A recent US-based study showed significant cost savings when adopting such a cutoff. ${ }^{33}$

Regarding location, our data support proximal location as a possible risk factor for PPB risk and clipping efficacy. In the control group, we observed a 2-fold increase in the risk for PPB for polyps located in the distal vs the proximal colon-from $2.7 \%$ to $6.2 \%$, respectively. Regarding the interaction between size and location, size $\geq 20 \mathrm{~mm}$ appeared to be the primary determinant of PPB risk. Thus, the benefit of clipping was clear in large proximal lesions, but it was absent for small lesions, even when they were located in the proximal colon. On the other hand, the risk of PPB and clipping efficacy were somewhat intermediate-although not statistically significant-for large and distal lesions. This was mainly attributed to the lack of effect of clipping in a large study of $\geq 20$-mm lesions. ${ }^{26}$ However, this was not confirmed by other studies, generating some uncertainty about the benefit of prophylactic clipping for large distal lesions. Thus, the decision for large and distal lesions should be tailored, especially taking into consideration other patient and polyp risk factors for PPB, such as the use of antithrombotic agents or intraprocedural bleeding. The studies included in our analysis did not show significant heterogeneity or publication bias. This indicates that the effect is homogeneous across the different series, suggesting generalizability and reproducibility of the results. Of note, the only study that contributed to the slight heterogeneity on our primary endpoint ${ }^{23}$ included only small polyps, suggesting that such size inclusion criteria could explain the slight degree of heterogeneity, as confirmed by subsequent metaregression and subgroup results. In addition, the observation that the effect is orientated toward clipping efficacy in the vast majority of the cases strengthens the robustness of our observation. 
Our data pair with recent a cost-effectiveness analysis (using Medicare cost data and Centers for Medicare and Medicaid billing codes) focusing on the impact of adoption of routine prophylactic clipping in practice from a payer perspective, considering relevant patient- and polyp-specific factors. ${ }^{33}$ Considering that clips are expensive and their placement might be technically demanding, prophylactic clipping tailored for a subgroup of higher-risk lesions/patients would decrease in parallel both adverse events and costs. Our data support the use of clipping for any large proximal lesion, excluding its use for those $<20 \mathrm{~mm}$ and those located in the distal colon. On the other hand, the choice of clipping for large distal lesions is likely to be less effective and should be individualized according to patient characteristics as well. ${ }^{34}$

There are limitations to our study. First, the paucity of data about patients' comorbidities and the management of antithrombotic therapies before endoscopic resection in many of the included studies limited our ability to infer on this relevant topic. Thus, high-quality RCTs are needed to determine whether prophylactic clips should be suggested after resecting small $(<20 \mathrm{~mm})$ and distal lesions in patients receiving antithrombotic therapies. Second, some of the included studies were of low to moderate quality, limiting the quality of our recommendations. Furthermore, study limitations such as lack of blinding of outcome assessors could almost be considered as intrinsic to RCTs evaluating endoscopic procedures, yet they remain the best possible evidence. Also, several patients included in the analysis underwent multiple resections. In the case of bleeding, it is sometimes difficult to determine which polypectomy site was bleeding in patients who did not undergo colonoscopy. Furthermore, even in cases requiring endoscopy, the bleeding site may remain uncertain. Finally, we were not able to stratify data according to technical variables that might affect the risk of bleeding (and the effect of clipping), such as hemoclips or electrosurgical unit characteristics (Supplementary Table 7).

In conclusion, the results of our meta-analysis of RCTs do not support the routine use of prophylactic clipping for the prevention of PPB. However, clipping appeared to be beneficial in patients with large $(>20 \mathrm{~mm})$ and proximal lesions.

\section{Supplementary Material}

Note: To access the supplementary material accompanying this article, visit the online version of Gastroenterology at www.gastrojournal.org, and at https://doi.org/10.1053/ j.gastro.2020.03.051.

\section{References}

1. Zauber AG, Winawer SJ, O’Brien MJ, et al. Colonoscopic polypectomy and long-term prevention of colorectalcancer deaths. N Engl J Med 2012;366:687-696.

2. Zorzi M, Senore C, Da Re F, et al. Detection rate and predictive factors of sessile serrated polyps in an organised colorectal cancer screening programme with immunochemical faecal occult blood test: the EQuIPE study (Evaluating Quality Indicators of the Performance of Endoscopy). Gut 2017;66:1233-1240.

3. Nishihara R, Wu K, Lochhead $P$, et al. Long-term colorectal-cancer incidence and mortality after lower endoscopy. N Engl J Med 2013;369:1095-1105.

4. Ferlitsch M, Moss A, Hassan C, et al. Colorectal polypectomy and endoscopic mucosal resection (EMR): European Society of Gastrointestinal Endoscopy (ESGE) clinical guideline. Endoscopy 2017; 49:270-297.

5. Ko CW, Riffle S, Michaels L, et al. Serious complications within 30 days of screening and surveillance colonoscopy are uncommon. Clin Gastroenterol Hepatol 2010; 8:166-173.

6. Rathgaber SW, Wick TM. Colonoscopy completion and complication rates in a community gastroenterology practice. Gastrointest Endosc 2006;64:556-562.

7. Bahin FF, Rasouli KN, Byth K, et al. Prediction of clinically significant bleeding following wide-field endoscopic resection of large sessile and laterally spreading colorectal lesions: a clinical risk score. Am J Gastroenterol 2016;111:1115-1122.

8. Jaruvongvanich $\mathrm{V}, \quad$ Prasitlumkum N, Assavapongpaiboon B, et al. Risk factors for delayed colonic post-polypectomy bleeding: a systematic review and meta-analysis. Int J Colorectal Dis 2017;32:13991406.

9. Park $\mathrm{CH}$, Jung YS, Nam E, et al. Comparison of efficacy of prophylactic endoscopic therapies for postpolypectomy bleeding in the colorectum: a systematic review and network meta-analysis. Am J Gastroenterol 2016;14:1140-1147.

10. Boumitri C, Mir FA, Ashraf I, et al. Prophylactic clipping and post-polypectomy bleeding: a meta-analysis and systematic review. Ann Gastroenterol 2016;29:502508.

11. Nishizawa T, Suzuki H, Goto O, et al. Effect of prophylactic clipping in colorectal endoscopic resection: a meta-analysis of randomized controlled studies. United European Gastroenterol J 2017:5:859-867.

12. Shamseer L, Moher D, Clarke M, et al. Preferred reporting items for systematic review and meta-analysis protocols (PRISMA-P) 2015: elaboration and explanation. BMJ 2015;349:g7647.

13. The Paris endoscopic classification of superficial neoplastic lesions: esophagus, stomach, and colon: November 30 to December 1, 2002. Gastrointest Endosc 2003;58(6 Suppl):S3-S43.

14. R Development Core Team. R: a language and environment for statistical computing. Vienna, Austria: R Foundation for Statistical Computing, 2016.

15. Schwarzer G. meta: an R package for meta-analysis. R News 2007;7:40-45.

16. Viechtbauer W. Conducting meta-analyses in $\mathrm{R}$ with the metafor package. J Stat Softw 2010;36:1-48.

17. Higgins JPT, Green S, eds. Cochrane handbook for systematic reviews of interventions. Version 5.0.2. https:// handbook-5-1.cochrane.org/v5.0.2/. Updated September 2009. 
18. Viechtbauer W, Cheung MW. Outlier and influence diagnostics for meta-analysis. Res Synth Methods 2010; 1:112-125.

19. Shioji K, Suzuki Y, Kobayashi M, et al. Prophylactic clip application does not decrease delayed bleeding after colonoscopic polypectomy. Gastrointest Endosc 2003; 57:691-694.

20. Dokoshi T, Fujiya M, Tanaka K, et al. A randomized study on the effectiveness of prophylactic clipping during endoscopic resection of colon polyps for the prevention of delayed bleeding. Biomed Res Int 2015;2015:490272.

21. Mori H, Kobara H, Nishiyama N, et al. Simple and reliable treatment for post-EMR artificial ulcer floor with snare cauterization for 10- to 20-mm colorectal polyps: a randomized prospective study (with video). Surg Endosc 2015;29:2818-2824.

22. Zhang QS, Han B, Xu JH, et al. Clip closure of defect after endoscopic resection in patients with larger colorectal tumors decreased the adverse events. Gastrointest Endosc 2015;82:904-909.

23. Matsumoto M, Kato M, Oba K, et al. Multicenter randomized controlled study to assess the effect of prophylactic clipping on post-polypectomy delayed bleeding. Dig Endosc 2016;28:570-576.

24. Osada T, Sakamoto N, Ritsuno H, et al. Closure with clips to accelerate healing of mucosal defects caused by colorectal endoscopic submucosal dissection. Surg Endosc 2016;30:4438-4444.

25. Albéniz E, Álvarez MA, Espinós JC, et al. Clip closure after resection of large colorectal lesions with substantial risk of bleeding. Gastroenterology 2019;157:1213-1221.

26. Pohl H, Grimm IS, Moyer MT, et al. Clip closure prevents bleeding after endoscopic resection of large colon polyps in a randomized trial. Gastroenterology 2019; 157:977-984.

27. Feagins LA, Smith AD, Kim D, et al. Efficacy of prophylactic hemoclips in prevention of delayed postpolypectomy bleeding in patients with large colonic polyps. Gastroenterology 2019;157:967-976.

28. Rex DK. Prophylactic clip closure clarified: the question is not whether to clip, but when. Gastroenterology 2019; 157:1190-1192.

29. Thoguluva Chandrasekar V, Spadaccini M, Aziz M, et al. Cold snare endoscopic resection of nonpedunculated colorectal polyps larger than $10 \mathrm{~mm}$ : a systematic review and pooled-analysis. Gastrointest Endosc 2019;89:929_ 936.

30. Forbes N, Frehlich L, James MT, et al. Routine prophylactic endoscopic clipping is not efficacious in the prevention of delayed post-polypectomy bleeding: a systematic review and meta-analysis of randomized controlled trials. J Can Assoc Gastroenterol 2019;2:105-117.

31. Ayoub F, Westerveld DR, Forde JJ, et al. Effect of prophylactic clip placement following endoscopic mucosal resection of large colorectal lesions on delayed polypectomy bleeding: a meta-analysis. World J Gastroenterol 2019;25:2251-2263.

32. Mangira D, Ket SN, Majeed A, et al. Postpolypectomy prophylactic clip closure for the prevention of delayed postpolypectomy bleeding: a systematic review. JGH Open 2018;2:105-110.

33. Shah ED, Pohl H, Rex DK, et al. Routine prophylactic clip closure is cost saving after endoscopic resection of large colon polyps in a Medicare population. Gastroenterology 2020;158:1164-1166.

34. Albéniz E, Gimeno-García AZ, Fraile M, et al. Clinical validation of risk scoring systems to predict risk of delayed bleeding after EMR of large colorectal lesions. Gastrointest Endosc 2020;91:868-878.

Received December 26, 2019. Accepted March 24, 2020.

\section{Correspondence}

Address correspondence to: Marco Spadaccini, MD, Humanitas Research Hospital and University, Rozzano, Italy, Via Manzoni 56, 20089 Rozzano (Milano) Italy. e-mail: marco.spadaccini@humanitas.it; fax: +390282242595.

\section{Acknowledgments}

The authors thank all participants of the clinical trials for the transfer of the data of the included studies, without whose contribution this work could not have been carried out. Eduardo Albéniz received a grant from the "La Caixa/Caja Navarra" Foundation (ID 100010434; project PR15/11100006).

\section{CRediT Authorship Contributions}

Marco Spadaccini, MD (Conceptualization: Lead; Data curation: Lead Investigation: Lead; Methodology: Lead; Writing - original draft: Lead); Eduardo Albéniz, MD (Conceptualization: Lead; Data curation: Lead Investigation: Lead; Methodology: Lead; Writing - review \& editing: Lead); Heiko Pohl, MD (Conceptualization: Equal; Supervision: Equal; Writing review \& editing: Equal); Roberta Maselli, MD, PhD (Conceptualization: Equal; Supervision: Equal; Writing - review \& editing: Equal); Viveksandeep Thoguluva Chandrasekar, MD (Validation: Equal; Writing - review \& editing Equal); Loredana Correale, (Data curation: Lead; Formal analysis: Lead); Andrea Anderloni, MD, PhD (Supervision: Equal; Writing - review \& editing: Equal); Silvia Carrara, MD (Supervision: Equal; Writing - review \& editing: Equal); Alessandro Fugazza, MD (Supervision: Equal; Writing - review \& editing: Equal); Mineo Iwatate, MD (Supervision: Equal; Writing - review \& editing: Equal); Giulio Antonelli, MD (Conceptualization: Supporting; Methodology: Supporting); Matteo Badalamenti, MD (Supervision: Equal; Writing - review \& editing: Equal); Mónica Enguita-Germán, MD (Supervision: Equal; Writing - review \& editing: Equal); Marco Antonio Álvarez, MD (Supervision: Equal; Writing - review \& editing: Equal); Prateek Sharma, MD (Conceptualization: Equal; Supervision: Equal; Writing - review \& editing: Equal); Douglas K. Rex, MD (Conceptualization: Equal; Supervision: Equal; Writing - review \& editing: Equal); Cesare Hassan, MD, PhD (Conceptualization: Equal; Supervision: Lead; Writing - review \& editing: Lead); Alessandro Repici, MD (Conceptualization: Equal; Supervision: Lead; Writing - original draft: Lead)

\section{Conflicts of interest}

The authors disclose no conflicts. 


\section{Supplementary Material}

\section{Data Sources and Search Strategy}

We performed a comprehensive literature search in the PubMed, Embase and SCOPUS (up to October 25, 2019) electronic databases to identify studies evaluating the role of prophylactic clipping in preventing PPB. PROSPERO was searched for ongoing or recently completed systematic reviews. Electronic searches were supplemented by manual searches of references of included studies and review articles.

We identified studies using the following medical subject headings (MeSH) and the keywords clip, clipping, polypectomy, bleeding, and endoscopic resection. The search was restricted to the English language.

The MEDLINE search strategy was as follows: "(c(c("surgical instruments"[MeSH Terms] OR ("surgical"[All Fields] AND "instruments"[All Fields]) OR "surgical instruments"[All Fields] OR "clip"[All Fields]) AND polypectomy[All Fields]) OR (("surgical instruments"[MeSH Terms] OR ("surgical"[All Fields] AND "instruments"[All Fields]) OR "surgical instruments"[All Fields] OR "clip"[All Fields]) AND endosopic[All Fields] AND resection[All Fields])) OR (("surgical instruments"[MeSH Terms] OR ("surgical"[All Fields] AND "instruments"[All Fields]) OR "surgical instruments"[All Fields] OR "clip"[All Fields]) AND ("hemorrhage"[MeSH Terms] OR "hemorrhage"[All Fields] OR "bleeding"[All Fields]) AND ("prevention and control"[Subheading] OR ("prevention"[All Fields] AND "control"[All Fields]) OR "prevention and control"[All Fields] OR "prophylaxis"[All Fields]))) OR (clipping[All Fields] AND polypectomy[All Fields])) OR (clipping[All Fields] AND endosopic[All Fields] AND resection[All Fields])) OR (clipping[All Fields] AND ("hemorrhage"[MeSH Terms] OR "hemorrhage"[All Fields] OR "bleeding"[All Fields]) AND ("prevention and control"[Subheading] OR ("prevention"[All Fields] AND "control"[All Fields]) OR "prevention and control"[All Fields] OR "prophylaxis"[All Fields]))".

\section{Selection Process}

Two review authors (MS and RM) independently screened the titles and abstracts yielded by the search against the inclusion criteria. Full reports were obtained for all titles that appeared to meet the inclusion criteria or where there was any uncertainty. Review author pairs then screened the full text and abstract reports and decided whether these met the inclusion criteria. Disagreements were resolved through discussion of all authors. The reasons for excluding trials were recorded. Neither of the review authors was blinded to the journal titles or to the study authors or institutions. When there were multiple articles for a single study, we used the latest publication and supplemented it, if necessary, with data from the more complete version.

\section{Data Extraction}

Using standardized forms, 2 reviewers (MS and RM) extracted data independently and in duplicate from each eligible study. Reviewers resolved disagreements by discussion. Unresolved disagreements were resolved by 2 arbitrators ( $\mathrm{CH}$ and $\mathrm{AR}$ ). The following data were extracted for each study: the publication status, study design and location, number of centers involved, number of patients, patient characteristics (mean/median age, sex, antithrombotic therapies), number of all lesions, number of procedures, mean/median lesion size, lesion location (proximal, distal), lesion aspect and histology, adverse events (bleedings, perforation surgery), mean/median lesion size. The corresponding authors of the included studies were asked for missing data.

\section{Quality Assessment}

Quality was assessed by the Cochrane risk bias tool for randomized studies. Two reviewers (MS and RM) assessed quality measures for included studies, and discrepancies were adjudicated by collegial discussion. The overall quality of evidence was appraised by applying GRADE methodology $^{1}$ for the primary outcomes.

\section{Multilevel (Random-Effects) Model: Meta- analysis of Rates of PPB With Clipping in Comparison to Not Clipping}

The effect size of the meta-analysis was the transformed logit proportion of PPB. The current meta-analysis included studies reporting multiple effect sizes (1 for each study arm). Multiple effect sizes within the same study may be correlated, violating the assumption of statistical independence. Thus, we used a multilevel meta-analytic model, taking into account the potential clustering effects within studies. This approach adds random effects for each effect size within each study to the traditional random effects model. Therefore, there are 2 random effects: the first random effect represents the variability between effect sizes assessed in the same study (ie, between-outcomes or within-study variance), whereas the second random effect represents the variability among the effects observed in different studies (ie, the between-study variance). For model fitting, we used the rma.mv function in metafor package in $\mathrm{R}$ package. ${ }^{2}$

The random effects at the level of outcome and study were specified as a list of 1-sided formulas in the random argument of the rma.mv function. The REML method was used, and a compound symmetry structure for the random effects was assumed (ie, the correlation coefficient $\rho$ for the correlation between the different outcomes was constant across studies). The model assumed independent sampling errors of effect size estimates. This is an appropriate assumption because there was no overlap in the patients used to compute outcome data in the 2 study arms. Subsequently, the multilevel model was extended by including predictor variables of delayed bleeding events (ie, study arm, prevalence of large lesions, and proximal colon location) in an attempt to find variables that moderate the effect. We also considered appropriate interactions between these variables. Data are presented as ORs and 95\% CIs. 


\section{Supplementary References}

1. Guyatt G, Oxman AD, Akl EA, et al. GRADE guidelines: 1. Introduction-GRADE evidence profiles and summary of findings tables. J Clin Epidemiol 2011;64:383-394.
2. Viechtbauer W. (2010). Conducting meta-analyses in R with the metafor package. J Stat Softw, 2010;36(3). http://www.jstatsoft.org/v36/i03/. Accessed August 5, 2010.

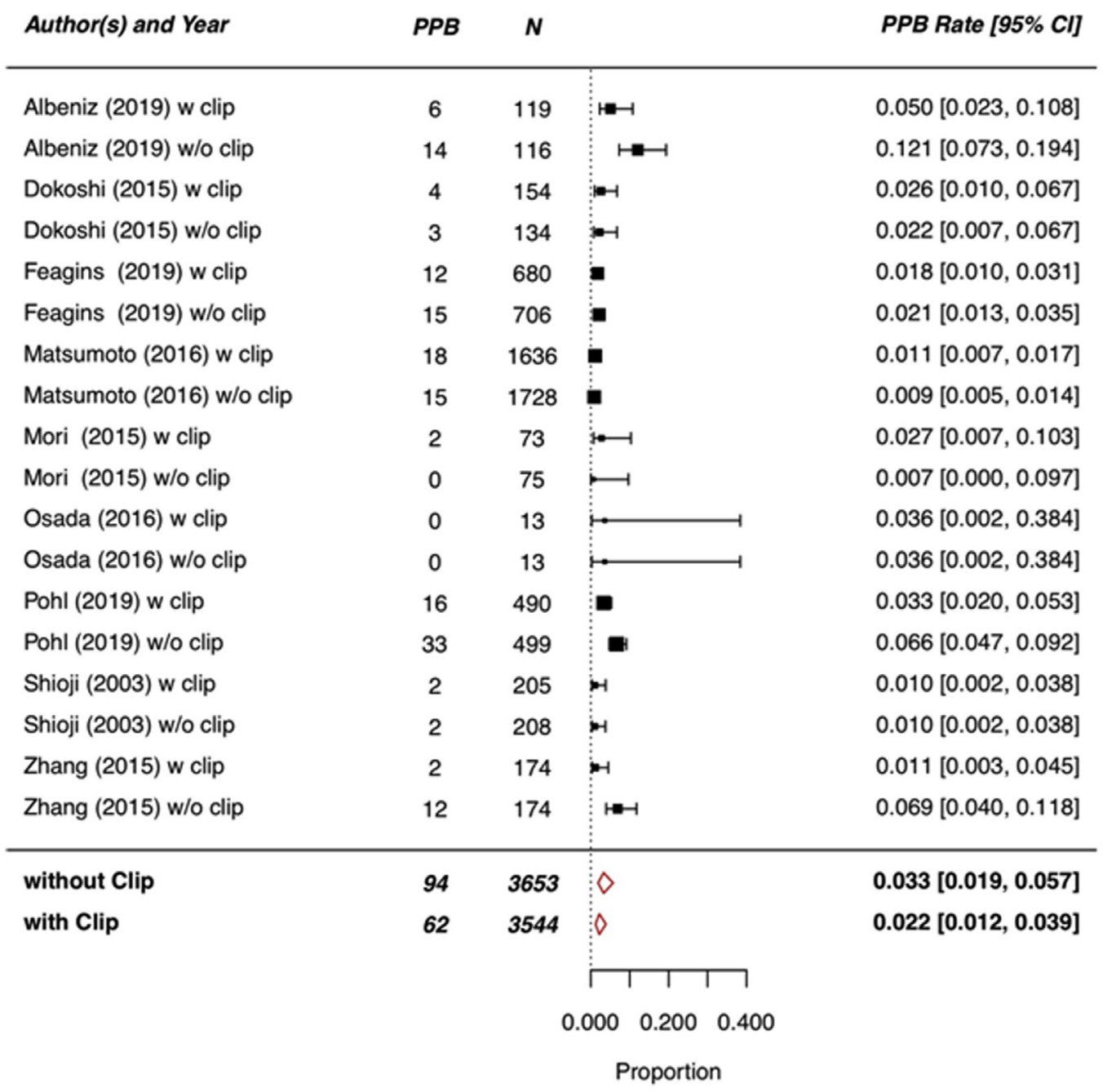

Supplementary Figure 1. Meta-Analysis of PPB rates of delayed bleeding-a multilevel random effects model accounting for the underlying heterogeneity between and within trials was used to estimate the pooled rates of PPB. 


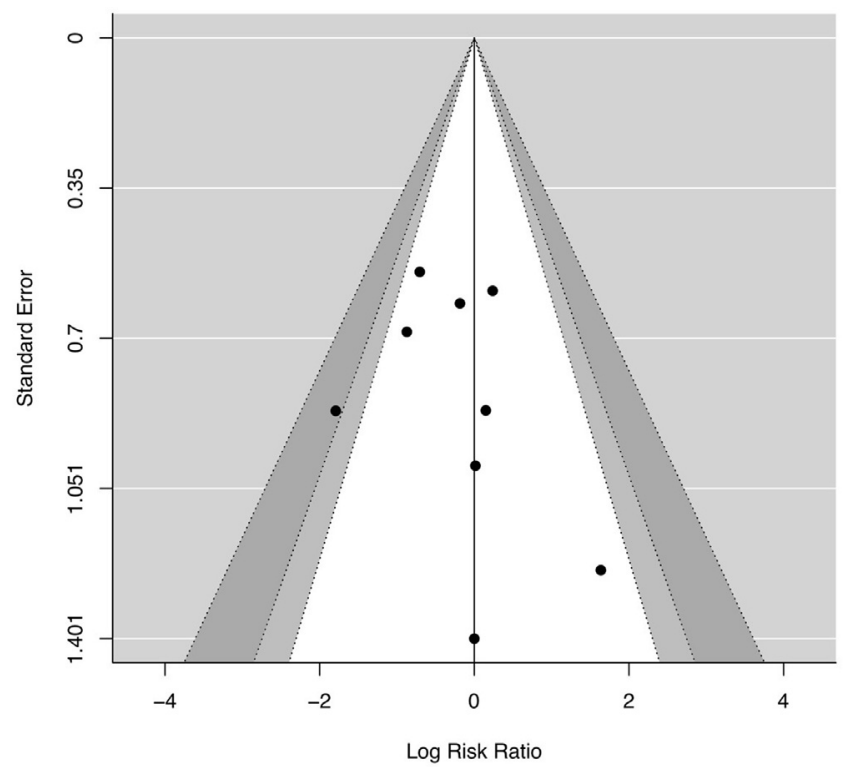

Supplementary Figure 2. Funnel plot of publication bias-ratio risk of PPB.

\section{Study}

Albeniz (2019)

Dokoshi (2015)

Feagins (2019)

Matsumoto (2016)

Mori (2015)

Osada (2016)

Pohl (2019)

Shioji (2003)

Zhang (2015)

Random effects model

Prediction interval

Heterogeneity: $I^{2}=33 \%, p=0.17$

\section{Clipped Unclipped} Events Total Events Total

$\begin{array}{rrrr}6 & 101 & 14 & 116 \\ 4 & 154 & 3 & 134 \\ . & . & . & . \\ 18 & 1636 & 15 & 1728 \\ 2 & 73 & 0 & 75 \\ 0 & 13 & 0 & 13 \\ 14 & 426 & 29 & 449 \\ 2 & 205 & 2 & 208 \\ 2 & 174 & 12 & 174\end{array}$

2782

2897

\section{RR [ $[95 \% \mathrm{Cl}]$}

$0.492 \quad[0.196 ; 1.233]$

$1.160 \quad[0.264 ; 5.091]$

$1.267 \quad[0.641 ; 2.507]$

$5.136[0.251 ; 105.174]$

$1.000 \quad[0.021 ; 46.836]$

$0.509 \quad[0.273 ; 0.950]$

$1.015 \quad[0.144 ; 7.135]$

$0.167 \quad[0.038 ; 0.734]$

$0.695[0.413 ; 1.170]$

$[0.213 ; 2.268]$

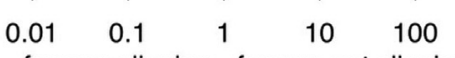

favours clipping favours not clipping

Supplementary Figure 3. Meta-analysis of relative risk of PPB-results from per protocol analysis (ie, PPB after completion of clipping). The study by Matsumoto et $\mathrm{al}^{23}$ had substantial influence on the pooled effect size estimate. 


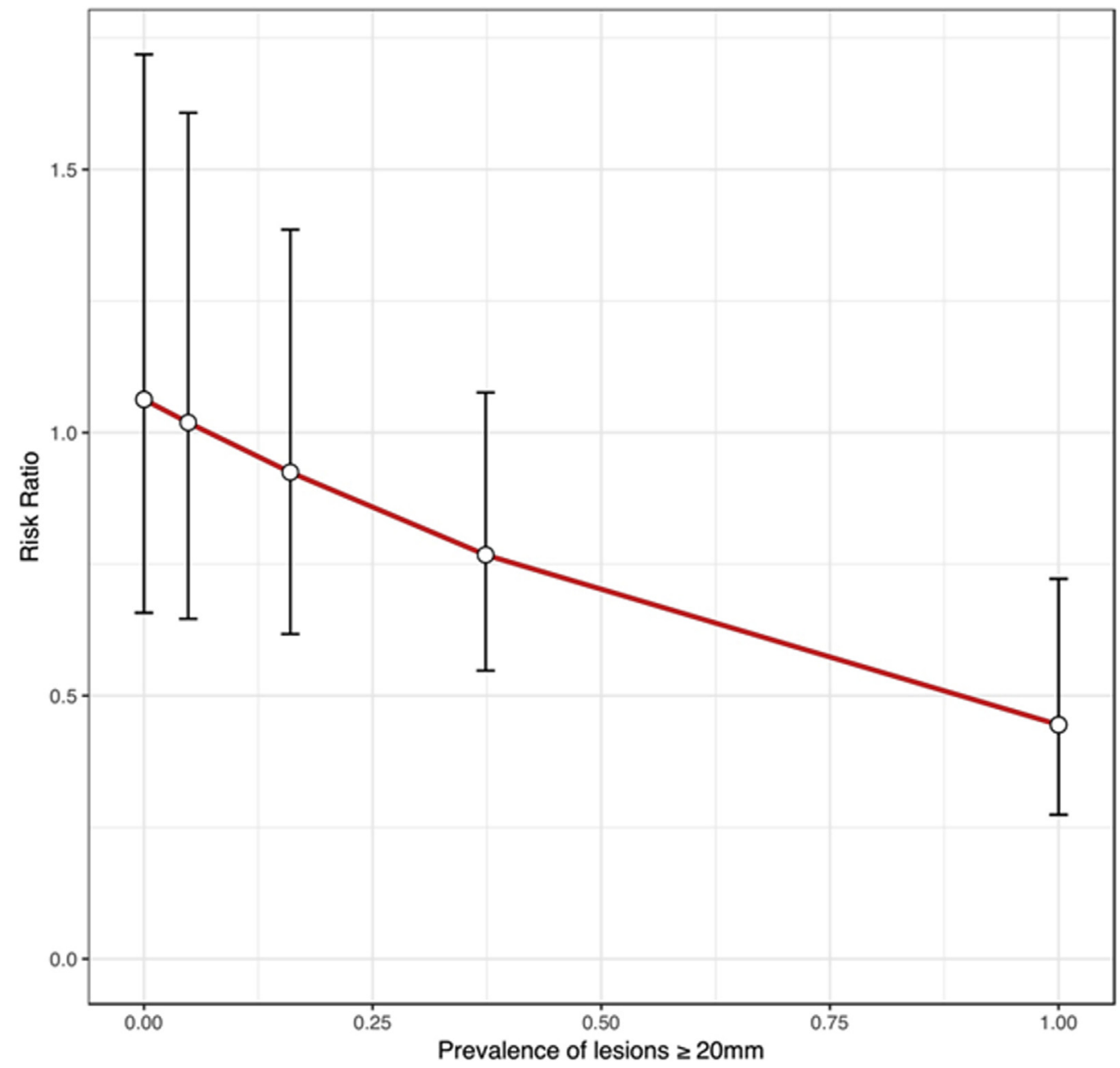

Supplementary Figure 4. A plot of the risk ratio estimate (derived from the metaregression model) as a function of the percentage (decimal number) of large lesions. The estimated risk ratio of PPB associated with clip use was not significantly different from 1 for a prevalence of large lesions of $0 \%$ (only small lesions included: RR, 1.06; 95\% Cl, 0.66-1.72), indicating an equal PPB risk, on average, for small clipped and unclipped polyps. However, we found increasingly large effects as the prevalence of large lesions increases. The estimated risk ratios of PPB for clipped (vs unclipped) polyps were 0.69 (95\% $\mathrm{Cl}$, $0.50-0.95)$ and $0.44(95 \% \mathrm{Cl}, 0.27-0.72)$ for percentages of large lesions of 0.50 and 1.00 , respectively. 


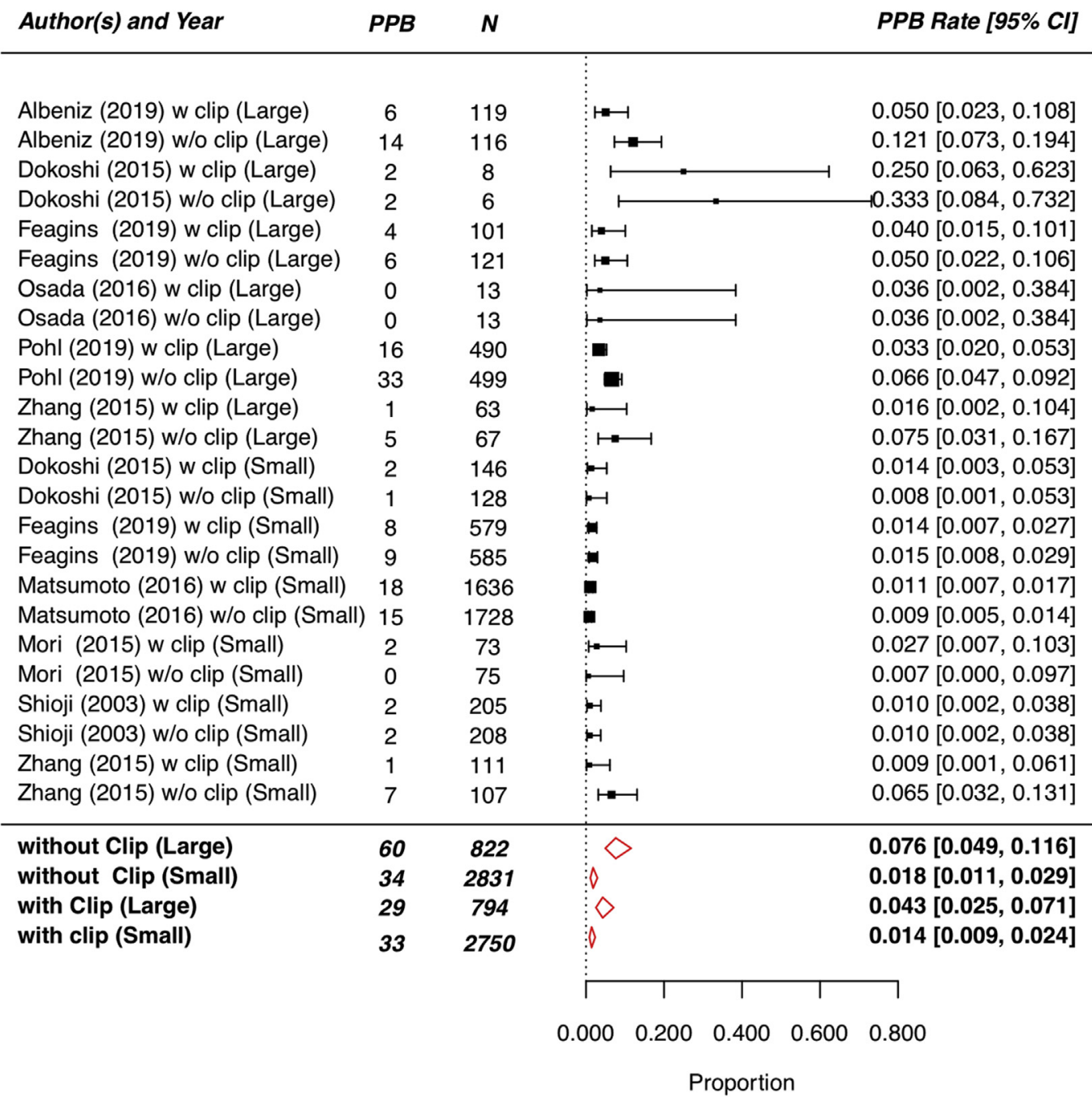

Supplementary Figure 5. Meta-analysis of PPB rates for large and small polyps. A multilevel random effects model accounting for the underlying heterogeneity between and within trials was used to estimate the rates of PPB for large and small lesions with and without clips. In our data set, 3 studies reported data on both large and small lesions, 3 studies only on large lesions, and the remaining 3 studies only on small lesions. Therefore, this analysis included 12 effect size estimates $(6$ for each study arm) of PPB among large polyps ( $n=1616$ polyps) and 12 effect size estimates (6 for each study arm) of PPB among small polyps ( $n=5581$ polyps).

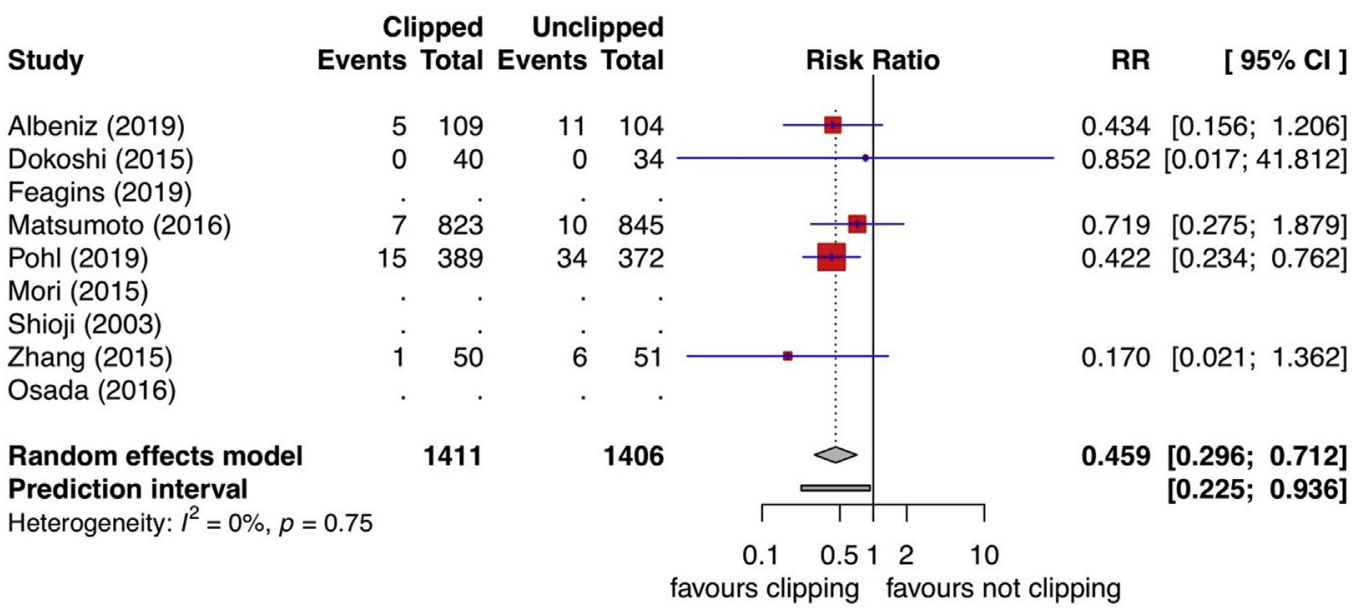

Supplementary Figure 6. Meta-analysis of relative risk of delayed bleeding-results from a sensitivity analysis among studies defining proximal lesions as sited proximally to the splenic flexure (including transverse colon). 


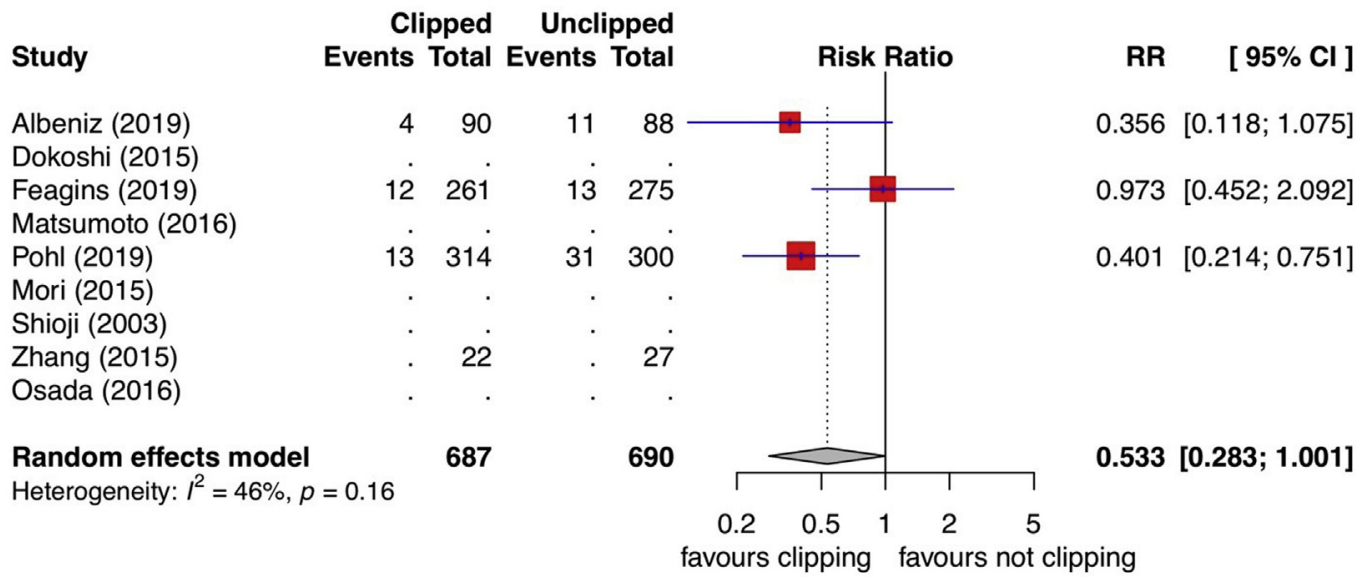

Supplementary Figure 7. Meta-analysis of relative risk of delayed bleeding-results from a sensitivity analysis among studies defining proximal lesions as lesions sited in cecum, ascending colon, and hepatic flexure.

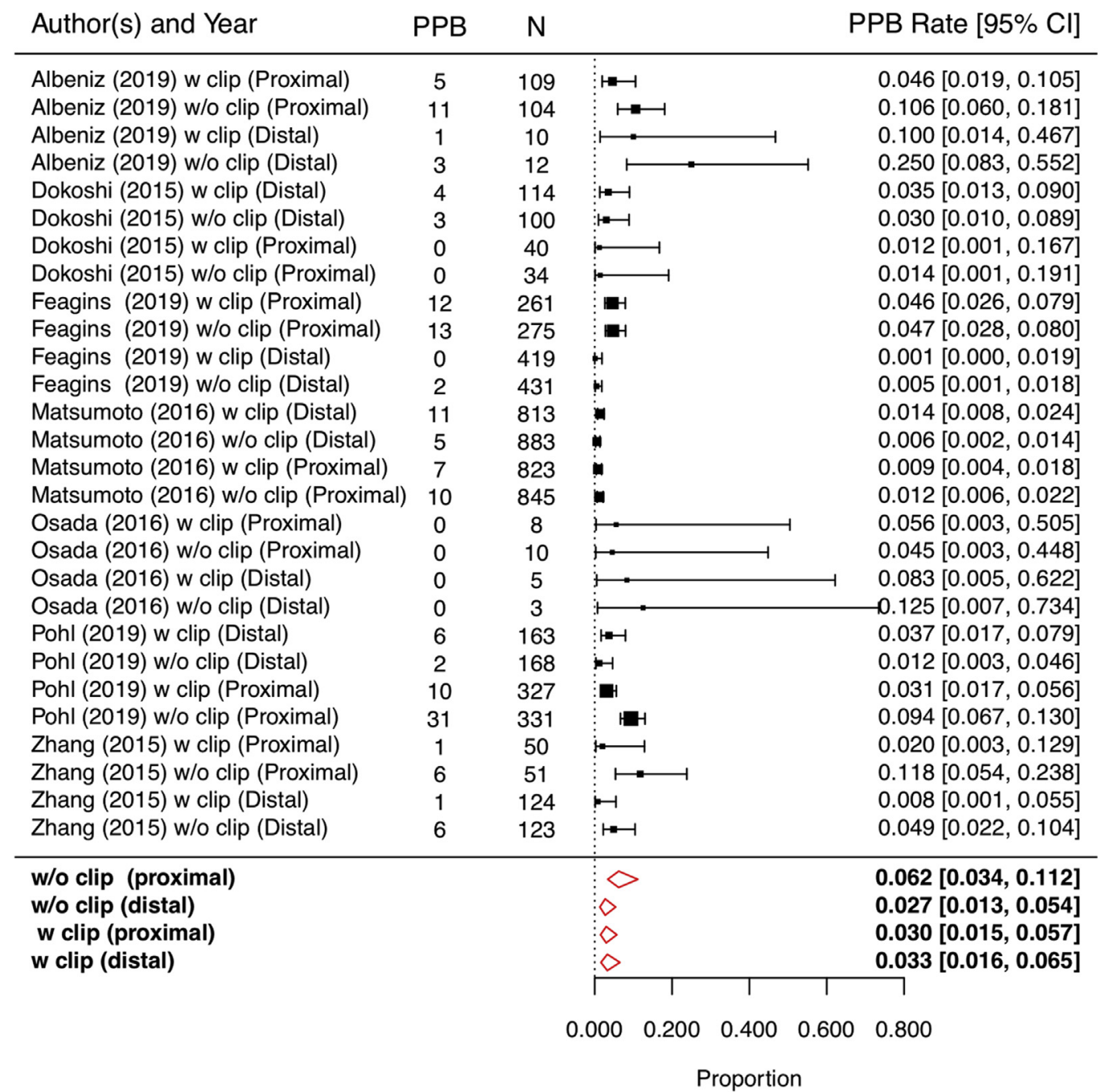

Supplementary Figure 8. Meta-analysis of PPB rates of delayed bleeding in the proximal colon location. A multilevel random effects model accounting for the underlying heterogeneity between and within trials was used to estimate the rates of PPB for proximally and distally located lesions with and without clips. The analysis included 7 studies $(n=6636$ polyps) with available count data for proximal $(n=3268)$ and distal lesions $(n=3368)$. Therefore, 14 effect size estimates $(7$ for each study arm) of PPB among distal and 14 effect size estimates (7 for each study arm) of PPB among proximal polyps were combined. 
Risk Ratio $[95 \% \mathrm{Cl}]$

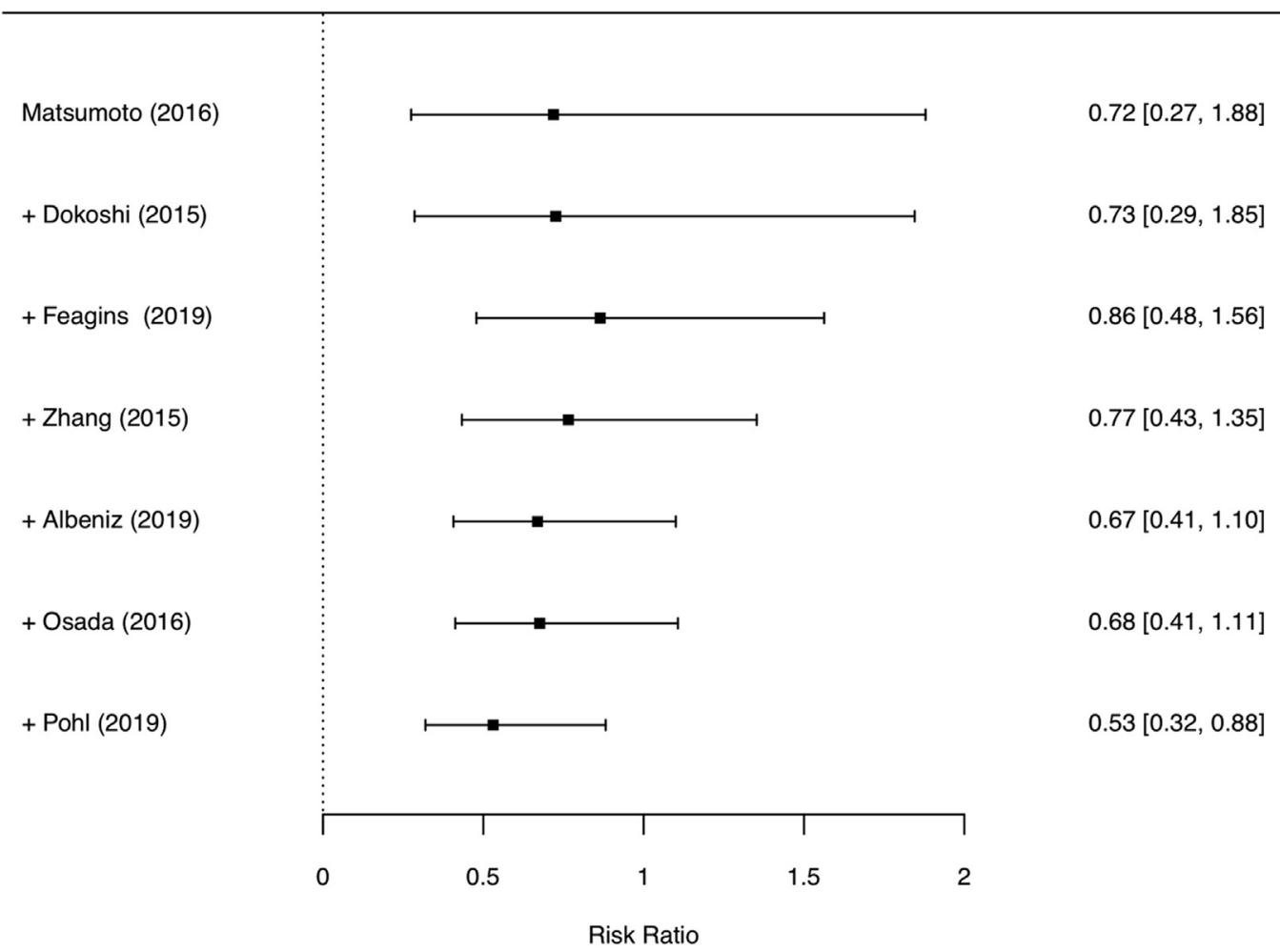

Supplementary Figure 9. Forest plot showing the results from a cumulative meta-analysis of the relative risk of PPB for clipped and unclipped polyps in the proximal colon. The cumulative meta-analysis was based on the study prevalence of large lesions and on a random effects model. The analysis shows how the overall estimate changes as studies with increasing prevalence of large lesions are added to the pool. Each study was added to the next one, and the summary effect was calculated at each step. Results from the cumulative meta-analysis suggest that studies with large lesions may overestimate the effect of clipping in lowering the risk of PPB in the proximal colon. 


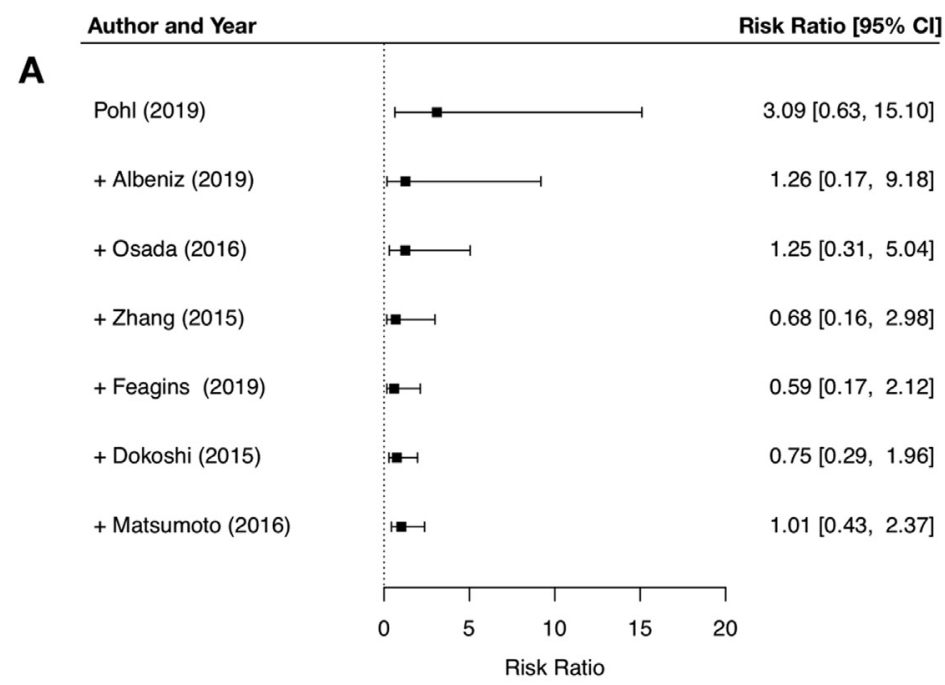

Author and Year

Risk Ratio [95\% Cl]

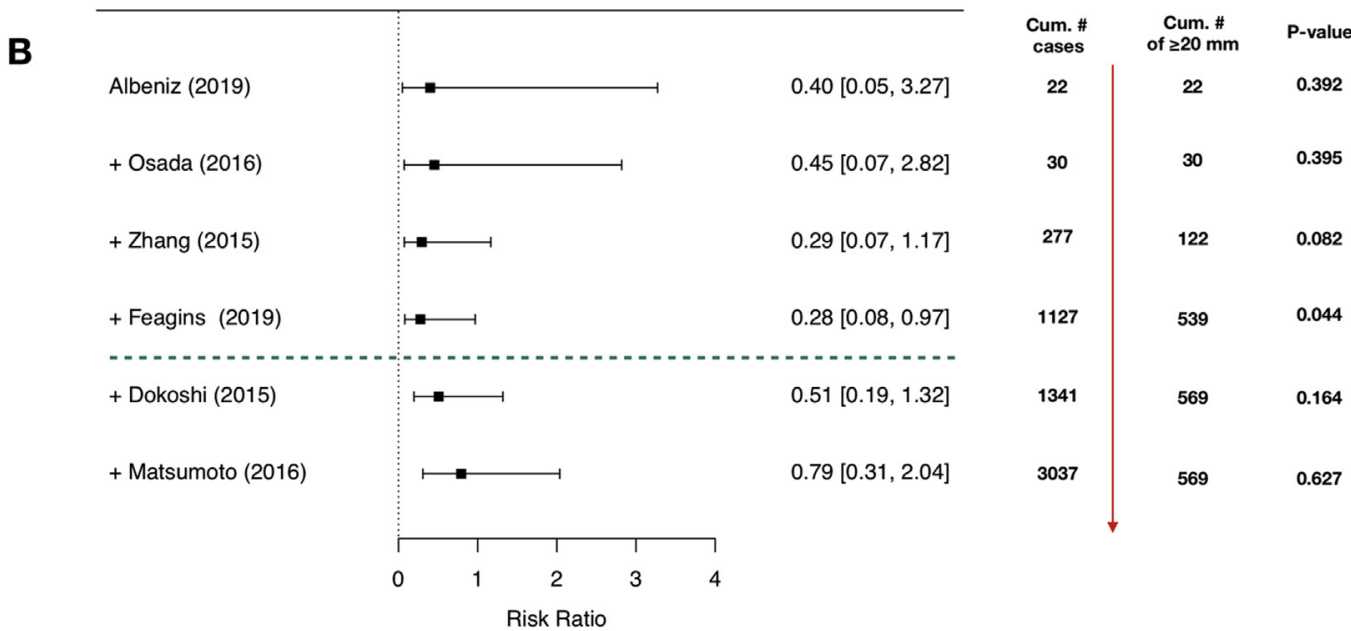

Supplementary Figure 10. (A) Forest plot showing the results from a cumulative meta-analysis of the relative risk of PPB for clipped and unclipped polyps in the distal colon. The cumulative meta-analysis was based on the study prevalence of large lesions (descending order) and on a random effects model. Results from the cumulative meta-analysis suggested that the study by Polh may underestimate the protective effect of clip for large distal lesions. (B) A sensitivity analysis excluding the study by Pohl et al. ${ }^{26}$ After excluding this study (in a sensitivity analysis), studies including large lesions showed a significant negative association between clip use and risk of PPB in the distal colon, supporting the hypothesis of a protective effect of clipping for large distal polyps (RR, $0.28 ; 95 \% \mathrm{Cl}, 0.08-0.97 ; P=.044)$. However, when studies reporting on small lesions were successively added to the analysis, the magnitude of the treatment effect began to increase and became nonsignificant. Cum \#, cumulative number of cases. 
Supplementary Table 1.Evaluation of Bias of RCTs Included in the Meta-analysis

\begin{tabular}{|c|c|c|c|c|c|c|c|}
\hline Study & $\begin{array}{l}\text { Random sequence } \\
\text { generation }\end{array}$ & $\begin{array}{l}\text { Allocation } \\
\text { concealment }\end{array}$ & $\begin{array}{l}\text { Blinding of participants } \\
\text { and personnel }\end{array}$ & $\begin{array}{l}\text { Blinding of outcome } \\
\text { assessment }\end{array}$ & $\begin{array}{l}\text { Adequate assessment } \\
\text { of incomplete outcome }\end{array}$ & $\begin{array}{l}\text { Selective } \\
\text { reporting avoided }\end{array}$ & No other bias \\
\hline Albéniz ${ }^{25}$ (2019) & Low risk & Low risk & High risk & High risk & Low risk & Low risk & Low risk \\
\hline Dokoshi et al ${ }^{20}(2015)$ & Unclear risk & Low risk & High risk & High risk & Low risk & Low risk & Low risk \\
\hline Feagins et $\mathrm{al}^{27}$ (2019) & Low risk & Low risk & High risk & High risk & Low risk & Low risk & Low risk \\
\hline Matsumoto et $\mathrm{al}^{23}$ (2016) & Low risk & Unclear risk & High risk & High risk & Low risk & Low risk & Low risk \\
\hline Mori et $\mathrm{al}^{21}(2015)$ & Low risk & Low risk & High risk & High risk & Low risk & Low risk & Low risk \\
\hline Osada et $\mathrm{al}^{24}(2016)$ & Unclear risk & Low risk & High risk & High risk & Low risk & Low risk & Low risk \\
\hline Pohl et $\mathrm{al}^{26}$ (2019) & Low risk & Low risk & High risk & High risk & Low risk & Low risk & Low risk \\
\hline Shioji et al ${ }^{19}$ (2003) & Unclear risk & Low risk & High risk & High risk & Low risk & Low risk & Low risk \\
\hline Zhang et al ${ }^{22}(2015)$ & Unclear risk & Low risk & High risk & High risk & Low risk & Low risk & Low risk \\
\hline
\end{tabular}

Supplementary Table 2. Meta-analysis of By-Polyp Delayed PBE Events and Clips Using Leave-One-Out Sensitivity

\begin{tabular}{|c|c|c|c|c|c|c|c|c|}
\hline & \multicolumn{4}{|c|}{ Influential analysis (fixed effects model) } & \multicolumn{4}{|c|}{ Influential analysis random effects model) } \\
\hline & $\mathrm{RR}(95 \% \mathrm{Cl})$ & $P$ value & $\operatorname{tau}^{2}$ & $P^{2}, \%$ & $\mathrm{RR}(95 \% \mathrm{Cl})$ & $P$ value & $\operatorname{tau}^{2}$ & $l^{2}, \%$ \\
\hline Omitting Albéniz ${ }^{25}$ (2019) & $0.72(0.51-1.00$ & .051 & 0.140 & 32.10 & $0.76(0.46-1.23)$ & .265 & 0.140 & 32.09 \\
\hline Omitting Dokoshi et $\mathrm{al}^{20}(2015)$ & $0.65(0.47-0.90)$ & .010 & 0.155 & 37.00 & $0.66(0.41-1.08)$ & .096 & 0.155 & 36.94 \\
\hline Omitting Feagins et $\mathrm{al}^{27}(2019)$ & $0.64(0.45-0.91)$ & .012 & 0.200 & 38.20 & $0.67(0.39-1.14)$ & .145 & 0.199 & 38.12 \\
\hline Omitting Matsumoto et $\mathrm{al}^{23}$ (2016) & $0.56(0.39-0.803)$ & .002 & 0.031 & 8.40 & $0.58(0.39-0.88)$ & .009 & 0.031 & 8.28 \\
\hline Omitting Mori et $\mathrm{al}^{21}$ (2015) & $0.65(0.47-0.89)$ & .008 & 0.104 & 29.40 & $0.67(0.43-1.02)$ & .064 & 0.104 & 29.39 \\
\hline Omitting Osada et $\mathrm{al}^{24}(2016)$ & $0.67(0.49-0.92)$ & .013 & 0.162 & 39.50 & $0.69(0.43-1.10)$ & .122 & 0.161 & 39.44 \\
\hline Omitting Pohl et $\mathrm{al}^{26}$ (2019) & $0.77(0.52-1.11)$ & .163 & 0.147 & 28.60 & $0.77(0.46-1.29)$ & .320 & 0.147 & 28.51 \\
\hline Omitting Shioji et $\mathrm{al}^{19}$ (2003) & $0.66(0.48-0.91)$ & .012 & 0.163 & 38.90 & $0.68(0.42-1.10)$ & .115 & 0.162 & 38.84 \\
\hline Omitting Zhang et $\mathrm{al}^{22}$ (2015) & $0.74(0.536-1.03)$ & .077 & 0.033 & 11.00 & $0.75(0.52-1.09)$ & .137 & 0.032 & 10.92 \\
\hline Pooled estimate & $0.67(0.49-0.92)$ & .013 & 0.126 & 31.10 & $0.69(0.45-1.08)$ & .072 & 0.126 & 27.54 \\
\hline
\end{tabular}


Supplementary Table 3. Multilevel Meta-regression Analysis of PPB Events

\begin{tabular}{llr}
\hline \multicolumn{1}{c}{ Unclipped polyps (reference level) } & OR & $95 \% \mathrm{Cl}$ \\
\hline & 1 & $P$ value \\
Clipped polyps & 1.06 & $0.66-1.75$ \\
Percentage of large lesions (as a continuous variable) & 1.28 & $1.16-1.42$ \\
Use of clip $\times$ percentage of large lesions & 0.92 & $0.85-0.98$ \\
Percentage of proximal lesions (as a continuous variable) & 0.86 & $0.67-1.10$ \\
\end{tabular}

Supplementary Table 4. A Multilevel Random Effects Model With Study Arm, Polyp Lesion (Large vs Small Polyps), and Their Interaction Term Fitted to the Data

\begin{tabular}{lccr}
\hline & & \multicolumn{2}{c}{ Multilevel meta-regression analysis } \\
\cline { 2 - 3 } & ORs & $95 \% \mathrm{Cl}$ \\
\hline Clipped large vs unclipped large polyps & 0.54 & $(0.30-0.97)$ & .041 \\
Unclipped small polyps vs unclipped large polyps & 0.22 & $(0.12-0.40)$ & $0.43-1.44$ \\
Clipped small vs unclipped small polyps & 0.79 & $(0.19-0.66)$ & .435 \\
Clipped small vs clipped large polyps & 0.36 & $<.001$ &
\end{tabular}

NOTE. The 2-way interaction term between polyp size and study arm was statistically significant. Results from the model show a protective effect of clipping for large polyps (clipped large vs unclipped large: OR, 0.54; 95\% Cl, 0.30-0.97), but no protective effect of clipping was seen among small polyps (clipped small vs unclipped small: $\mathrm{OR}, 0.79 ; 95 \% \mathrm{Cl}, 0.43-1.44 ; P=.435$ ).

Supplementary Table 5. A Multilevel Random Effects Model With Study Arm, Proximal Colon Location, and Their Interaction Term Fitted to the Data

\begin{tabular}{lccc}
\hline & & Multilevel Metaregression analysis \\
\cline { 2 - 3 } & ORs & $95 \% \mathrm{Cl}$ \\
\hline Clipped proximal vs unclipped proximal polyps & 0.46 & $(0.24-0.88)$ & .0018 \\
Unclipped distal vs unclipped proximal polyps & 0.42 & $(0.24-0.73)$ & $(0.61-1.95)$ \\
Clipped distal vs clipped proximal polyps & 1.09 & $0.56-1.38$ & .764 \\
Clipped distal vs unclipped distal polyps & 1.21 & .631 \\
\hline
\end{tabular}

NOTE. The 2-way interaction term between colon location and study arm was statistically significant $(P=.017)$. Results from the model show a protective effect of clipping for proximal polyps (clipped proximal vs unclipped proximal polyps: OR, 0.46; $95 \% \mathrm{Cl}, 0.24-0.88$ ), but no protective effect of clipping was seen among distal polyps (clipped distal vs unclipped distal polyps: OR, $1.21 ; 95 \% \mathrm{Cl}, 0.56-2.62 ; P=.631$ ). After adjusting for the prevalence of large lesions in the multilevel model, the benefit of clipping in reducing PPB was significant only for large proximal lesions (clipped vs unclipped polyps: OR, 0.34; 95\% $\mathrm{Cl}, 0.19-0.65 ; P=.021$ ) but not for small proximal lesions (clipped vs unclipped polyps: $\mathrm{OR}, 0.88$; $95 \% \mathrm{Cl}, 0.48-1.62$; $P=.581)$. 
Outcome, number of studies,

Quality assessment

Summary of findings

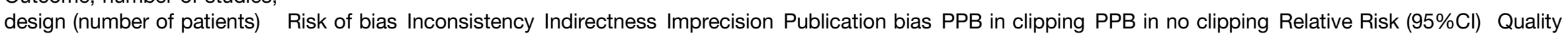

\begin{tabular}{|c|c|c|c|c|c|c|c|c|c|}
\hline $\begin{array}{l}\text { All lesions, } \\
9 \text { RCTs }\end{array}$ & Serious $^{a}$ & Serious $^{b}$ & Not serious & Not serious & Not serious & $62 / 3544$ & $94 / 3653$ & $0.69(0.45-1.08)$ & $\begin{array}{l}\oplus \oplus \mathrm{OO} \\
\text { Low }\end{array}$ \\
\hline $\begin{array}{l}\text { Large (>20 mm) lesions, } \\
6 \text { RCTs }\end{array}$ & Serious $^{a}$ & Not serious & Not serious & Not serious & Not serious & $29 / 794$ & $60 / 822$ & $0.51(0.33-0.78)$ & $\begin{array}{l}\oplus \oplus \oplus \mathrm{O} \\
\text { Moderate }\end{array}$ \\
\hline $\begin{array}{l}\text { Proximal lesions, } \\
7 \text { RCTs }\end{array}$ & Serious $^{a}$ & Not serious & Not serious & Not serious & Not serious & $35 / 1618$ & $71 / 1650$ & $0.53(0.35-0.81)$ & $\begin{array}{l}\oplus \oplus \oplus \mathrm{O} \\
\text { Moderate }\end{array}$ \\
\hline
\end{tabular}

${ }^{a}$ Risk of bias was judged as serious because of the low-moderate quality of the included randomized controlled trials.

${ }^{b}$ Inconsistency risk was judged as serious because of the heterogeneity among patients (ie, different indications to resection in the term of lesion size or location).

Supplementary Table 7.Technical Features

\begin{tabular}{|c|c|c|}
\hline Study & Electrosurgical generators & Clips \\
\hline Albéniz ${ }^{25}$ (2019) & Erbe (ICC200, VIO 200 or VIO300), Endocut mode & Micro-Tech, Nanjing, China \\
\hline Dokoshi et $\mathrm{al}^{20}(2015)$ & ERBE (ICC200 or VIO300), Endocut mode & HX-610-135, Olympus, Tokyo, Japan \\
\hline Feagins et $\mathrm{al}^{27}(2019)$ & NA & NA \\
\hline Matsumoto et $\mathrm{al}^{23}(2016)$ & Erbe (ICC200 or VIO200), Olympus (ESG-100, PSD.60) & NA \\
\hline Mori et $\mathrm{al}^{21}(2015)$ & Erbe (VIO300); Swift mode & HX-610-135, Olympus \\
\hline Osada et $\mathrm{al}^{24}(2016)$ & Erbe (VIO300), Endocut mode & ZEOCLIP ZP-CH, ZeonMedical, Tokyo, Japan \\
\hline Pohl et $\mathrm{al}^{26}(2019)$ & Erbe generators, Endocut or forced coagulation modes & $\begin{array}{l}\text { Resolution clip, Boston Scientific, Marlborough, Massachusetts } \\
\text { Resolution } 360 \text { clip, Boston Scientific }\end{array}$ \\
\hline Shioji et $\mathrm{al}^{19}$ (2003) & Olympus (UES-10), blended current & HX-5QR-1, Olympus \\
\hline Zhang et $\mathrm{al}^{22}$ (2015) & ERBE (ICC200 or VIO300); Endocut mode & $\begin{array}{l}\text { HX-610-135, Olympus } \\
\text { Resolution clip, Boston Scientific, Natic, Massachusetts }\end{array}$ \\
\hline
\end{tabular}

NA, not available. 
Este artículo ha sido eliminado por restricciones de derechos de autor.

Albéniz, E., Enguita-Germán, M., Gimeno-García, A. Z., Herreros de Tejada, A., Nogales, O., Espinós, J. C., Rodríguez Sánchez, J., Rosón, P., Guarner, C., Marín, J. C., Bhandari, P., Spadaccini, M., Repici, A., Hassan, C., Álvarez-González, M. A., \& Ibáñez Beroiz, B. (2021). The answer to "when to clip" after colorectal endoscopic mucosal resection based on a cost-effectiveness analysis. American Journal of Gastroenterology, 116(2), 311-318. https://doi.org/10.14309/ajg.0000000000000943 